\title{
Ugi reaction synthesis of oxindole-lactam hybrids as selective butyrylcholinesterase inhibitors
}

Pedro Brandão ${ }^{1,2}$, Óscar López, ${ }^{3}$ Luisa Leitzbach, ${ }^{4}$ Holger Stark, ${ }^{4}$ José G. Fernández-Bolaños, ${ }^{3}$ Anthony J. Burke, ${ }^{2,5}$ Marta Pineiro ${ }^{1 *}$

1 University of Coimbra, CQC and Department of Chemistry, 3004-535 Coimbra, Portugal

2LAQV-REQUIMTE, Rua Romão Ramalho, 59, University of Évora, 7000 Évora, Portugal

${ }^{3}$ Departamento de Química Orgánica, Facultad de Química, Universidad de Sevilla, Apartado 1203, E-41071 Seville, Spain

${ }^{4}$ Institute of Pharmaceutical and Medicinal Chemistry, Heinrich Heine University Düsseldorf, Universitaetsstr. 1, 40225 Duesseldorf, NRW, Germany

5University of Evora, Department of Chemistry, Rua Romão Ramalho, 59, 7000 Évora, Portugal

\section{Table of Contents:}

1. Experimental

Page

1.1. General procedure for the synthesis of $\beta$-lactam-oxindole hybrids

1.2. General procedure for the synthesis of $y$-lactam-oxindole hybrids

S1

1.3. Cholinesterase evaluation assay

1.4. Monoamine oxidases inhibition assay

$\mathrm{S} 11$

2. NMR spectra

$\mathrm{S} 11$

2.1. ${ }^{1} \mathrm{H}$ and ${ }^{13} \mathrm{C}$ NMR spectra of $\beta$-lactam-oxindole hybrids

$\mathrm{S} 13$

2.2. ${ }^{1} \mathrm{H}$ and ${ }^{13} \mathrm{C}$ NMR spectra of $\mathrm{Y}$-lactam-oxindole hybrids

$\mathrm{S} 13$

3. High-Resolution Mass Spectra

S30

3.1. High-Resolution Mass Spectra of $\beta$-lactam-oxindole hybrids

S44

3.2. High-Resolution Mass Spectra of $\gamma$-lactam-oxindole hybrids

S44

4. ATR-FTIR spectra

S52

4.1. ATR-FTIR Spectra of $\beta$-lactam-oxindole hybrids $\quad$ S59

4.2. ATR-FTIR Spectra of $y$-lactam-oxindole hybrids $\quad S 68$

5. SwissADME $\circledast$ evaluation reports $\quad S 75$

6. References 


\section{Experimental}

All commercial reagents were obtained from Sigma-Aldrich, Acros, Alfa Aesar and Fluorochem and used without further purification. The solvents were purified using standard laboratory practices. All reactions were monitored by thin-layer chromatography (TLC), using aluminum-backed kieselgel 60 F254 plates (Merck); spots were visualized with UV light or by treatment with suitable TLC stains. Column chromatography was performed using silica gel 60 (Merck 7-230 mesh). Melting points were determined using a Melting Point Device Falc R132467 (open capillary method). ${ }^{1} \mathrm{H}$ and ${ }^{13} \mathrm{C}$ NMR spectra were acquired on a Bruker Avance III or on a Varian Unity Plus VXR operating at $400 \mathrm{MHz}\left({ }^{1} \mathrm{H}\right)$ and $100.62 \mathrm{MHz}\left({ }^{13} \mathrm{C}\right)$. The chemical shift data were reported in ppm units $(\delta)$ relative to the residual solvent; coupling constants $(J)$ are reported in hertz and refer to apparent peak multiplicities. High-resolution mass spectra (HRMS) were recorded on a Mass spectrometer MICRO-MASS Q-TOF (Waters), or on a TOF VG Autospect M spectrometer with electrospray ionization (ESI), or on a Orbitrap Q-exactive focus (Thermo scientific) coupled with a HPLC vanquish (thermo scientific) with ESI.

\subsection{General procedure for the synthesis of $\beta$-lactam-oxindole hybrids} obtained via U4c3CR (Library 4). To a stirring solution of isatin derivative (100 mg, 1.0 equiv) and 3-aminopropanoic acid (1.0 equiv) in TFE ( $1 \mathrm{~mL}$ ), isocyanide (1.0 equiv) was added. The reaction was allowed to stir at room temperature and the progress monitored by TLC. Upon completion, the solvent was evaporated under reduced pressure and the crude reaction mixture was purified by column chromatography (CC) using the eluent indicated in each case. The structural elucidation and purity evaluation was performed using NMR, HRMS, ATR-FTIR and melting point determination.

\section{N-(tert-Butyl)-2-oxo-3-(2-oxoazetidin-1-yl)indoline-3-carboxamide (4aaa):}

Prepared using isatin, 3-aminopropanoic acid and tert-butyl isocyanide. CC: Hexane:EtOAc 2:1. The corresponding 4aaa was obtained as a beige solid (165.2 mg, $81 \%$ yield). m.p. $=173.0-174.2{ }^{\circ} \mathrm{C} .{ }^{1} \mathrm{H}$ NMR $\left(\mathrm{CDCl}_{3}, 400 \mathrm{MHz}\right): \delta 8.61$ (br s, $\left.1 \mathrm{H}\right), 7.66(\mathrm{~d}$, $J=8 \mathrm{~Hz}, 1 \mathrm{H}), 7.35$ (br s, 1H), $7.26(\mathrm{t}, \mathrm{J}=8 \mathrm{~Hz}, 1 \mathrm{H}), 7.09(\mathrm{t}, \mathrm{J}=8 \mathrm{~Hz}, 1 \mathrm{H}), 6.83(\mathrm{~d}, \mathrm{~J}=8 \mathrm{~Hz}$, $1 \mathrm{H}), 3.62-3.59(\mathrm{~m}, 1 \mathrm{H}), 3.42-3.38(\mathrm{~m}, 1 \mathrm{H}), 2.96-2.92(\mathrm{~m}, 2 \mathrm{H}), 1.37(\mathrm{~s}, 9 \mathrm{H}) .{ }^{13} \mathrm{C} N M R$ $\left(\mathrm{CDCl}_{3}, 100 \mathrm{MHz}\right): \delta 172.6,167.3,162.1,140.7,130.1,126.6,126.0,123.4,110.8,68.2$, $52.3,39.5,35.9,28.5$. Compound previously described in the literature. ${ }^{1}$

\section{N-(tert-Butyl)-1-methyl-2-oxo-3-(2-oxoazetidin-1-yl)indoline-3-carboxamide} (4baa): 
Prepared using $\mathrm{N}$-methylisatin, 3-aminopropanoic acid and tert-butyl isocyanide. CC: Hexane:EtOAc 2:1. The corresponding 4baa was obtained as a white solid (128.7 mg, $66 \%$ yield). m.p.= 117.8-119.0 ${ }^{\circ} \mathrm{C} .{ }^{1} \mathrm{H}$ NMR $\left(\mathrm{CDCl}_{3}, 400 \mathrm{MHz}\right): \delta 7.72(\mathrm{~d}, \mathrm{~J}=8,1 \mathrm{H})$, 7.39-7.35 (m, 2H), $7.14(\mathrm{td}, J=8,4 \mathrm{~Hz}, 1 \mathrm{H}), 6.87(\mathrm{~d}, J=8 \mathrm{~Hz}, 1 \mathrm{H}), 3.54-3.51(\mathrm{~m}, 1 \mathrm{H})$, 3.35-3.31 (m, 1H), $3.27(\mathrm{~s}, 3 \mathrm{H}), 2.90(\mathrm{t}, \mathrm{J}=4 \mathrm{~Hz}, 2 \mathrm{H}), 1.37(\mathrm{~s}, 9 \mathrm{H}) \cdot{ }^{13} \mathrm{C} \mathrm{NMR}\left(\mathrm{CDCl}_{3}, 100\right.$ $\mathrm{MHz}): \delta 171.4,166.9,162.1,143.3,130.1,126.7,125.4,123.7,108.8,68.0 .52 .2,39.2$, $36.0,28.5(3 \mathrm{C}), 26.8$. Compound previously described in the literature. ${ }^{1}$

N-(tert-Butyl)-2-oxo-3-(2-oxoazetidin-1-yl)-1-(prop-2-yn-1-yl)indoline-3carboxamide (4caa):

$N$-propargylisatin was synthesized as described in the literature. ${ }^{2}$ Briefly, to a solution of isatin ( $1.0 \mathrm{~g}, 1$ equiv) in DMF kept at $0 \stackrel{\circ}{\circ}$, sodium hydride ( 1.5 equiv) was slowly added and allowed to stir until the gas production ceased. Then, propargyl bromide (1.4 equiv) was added dropwise and the mixture was allowed to proceed with stirring at room temperature overnight. The DMF was then removed under reduced pressure. Water was added and the aqueous mixture extracted with ethyl acetate $(3 \mathrm{x}$ $30 \mathrm{~mL}$ ), and the combined organic layers were washed with brine, dried with anhydrous $\mathrm{Na}_{2} \mathrm{SO}_{4}$ and evaporated to afford the desired product, which was used in the next step without further purification. The lactam-oxindole hybrid 4caa was prepared using $\mathrm{N}$ propargylisatin, 3-aminopropanoic acid and tert-butyl isocyanide. CC: Hexane:EtOAc 2:1. The corresponding 4caa was obtained as a beige solid $(124.0 \mathrm{mg}, 68 \%$ yield). m.p.= 130.5-131.9 ํㅡ. ${ }^{1} \mathrm{H}$ NMR $\left(\mathrm{CDCl}_{3}, 400 \mathrm{MHz}\right): \delta 7.73(\mathrm{~d}, \mathrm{~J}=8 \mathrm{~Hz}, 1 \mathrm{H}), 7.39(\mathrm{t}, J=8 \mathrm{~Hz}, 1 \mathrm{H})$, 7.25 (br s, 1H), 7.17 (t, J=8 Hz, 1H), 7.09 (d, J=8, 1H), 4.54 (d, J=4 Hz, 2H), 3.54-3.51 (m, 1H), 3.33-3.29 (m, 1H), 2.91 (t, J=4 Hz, 2H), 2.27 (t, J=4 Hz, 1H), 1.37 (s, 9H). ${ }^{13} \mathrm{C}$ $\operatorname{NMR}\left(\mathrm{CDCl}_{3}, 100 \mathrm{MHz}\right): \delta$ 170.4, 167.2, 162.0, 141.5, 130.2, 126.7, 125.4, 124.0, 109.8, $76.1,72.9,68.3,52.3,39.1,36.0,29.9,28.5$ (3C). HRMS (ESI) calculated for $\mathrm{C}_{19} \mathrm{H}_{22} \mathrm{~N}_{3} \mathrm{O}_{3}$ $[\mathrm{MH}]^{+}$340.1656, found 340.1653; and calculated for $\mathrm{C}_{19} \mathrm{H}_{21} \mathrm{~N}_{3} \mathrm{NaO}_{3}[\mathrm{MNa}]^{+}$362.1475, found 362.1462 .

N-(tert-Butyl)-2-oxo-3-(2-oxoazetidin-1-yl)-1-phenylindoline-3-carboxamide (4daa):

Prepared using $\mathrm{N}$-phenylisatin, 3-aminopropanoic acid and tert-butyl isocyanide. CC: Hexane:EtOAc 2.5:1. The corresponding 4daa was obtained as a white solid (102.8 mg, 61\% yield). m.p.=180.2-181.5 $\stackrel{\circ}{ } \mathrm{C} .{ }^{1} \mathrm{H} \mathrm{NMR}\left(\mathrm{CDCl}_{3}, 400 \mathrm{MHz}\right): \delta 7.79$ (d, J=8 Hz, $1 \mathrm{H}), 7.57-7.53(\mathrm{~m}, 2 \mathrm{H}), 7.49-7.43(\mathrm{~m}, 4 \mathrm{H}), 7.28(\mathrm{td}, J=8,4 \mathrm{~Hz}, 1 \mathrm{H}), 7.17(\mathrm{t}, J=8 \mathrm{~Hz}, 1 \mathrm{H})$, $6.81(\mathrm{~d}, \mathrm{~J}=8 \mathrm{~Hz}, 1 \mathrm{H}), 3.64-3.60(\mathrm{~m}, 1 \mathrm{H}), 3.45-3.41(\mathrm{~m}, 1 \mathrm{H}), 3.00-2.90(\mathrm{~m}, 2 \mathrm{H}), 1.39$ (s, $9 \mathrm{H}) .{ }^{13} \mathrm{C} \mathrm{NMR}\left(\mathrm{CDCl}_{3}, 100 \mathrm{MHz}\right): \delta 171.0,166.8,161.8,143.5,133.7,130.0,129.8(2 \mathrm{C})$, 
128.7, 127.0, 126.7 (2C), 125.3, 124.0, 110.0, 67.7, 52.3, 39.2, 36.1, 28.5 (3C). HRMS (ESI) calculated for $\mathrm{C}_{22} \mathrm{H}_{23} \mathrm{~N}_{3} \mathrm{NaO}_{3}[\mathrm{MNa}]^{+} 400.1632$, found 400.1624 .

1-Benzyl-N-(tert-butyl)-2-oxo-3-(2-oxoazetidin-1-yl)indoline-3-carboxamide (4eaa):

Prepared using $\mathrm{N}$-benzylisatin, 3-aminopropanoic acid and tert-butyl isocyanide. CC: Hexane:EtOAc 1.5:1. The corresponding 4eaa was obtained as a white solid (125.7 mg, $76 \%$ yield). m.p. $=119.2-120.3 \stackrel{\circ}{\circ}$. ${ }^{1} \mathrm{H} \mathrm{NMR}\left(\mathrm{CDCl}_{3}, 400 \mathrm{MHz}\right): \delta 7.70$ (d, J=8 Hz, 1H), 7.35-7.21 (m, 7H), 7.10 (t, J=8 Hz, 1H), 6.71 (d, J=8 Hz, 1H), 5.03 (d, J=16 Hz, 1H), 4.89 (d, J=16 Hz, 1H), 3.60-3.57 (m, 1H), 3.38-3.34 (m, 1H), 2.94 (t, J=4, 2H), 1.38 (s, $9 \mathrm{H}) .{ }^{13} \mathrm{C} \mathrm{NMR}\left(\mathrm{CDCl}_{3}, 100 \mathrm{MHz}\right): \delta 171.6,167.0,162.0,142.3,134.9,130.0,128.9(2 \mathrm{C})$, 127.8, 127.2 (2C), 126.7, 125.6, 123.7, 109.9, 68.2, 52.2, 44.3, 39.3, 36.1, 28.5 (3C). Compound previously described in the literature. ${ }^{1}$

5-Bromo-N-(tert-butyl)-2-oxo-3-(2-oxoazetidin-1-yl)indoline-3-carboxamide (4faa):

Prepared using 5-bromoisatin, 3-aminopropanoic acid and tert-butyl isocyanide. CC: Hexane:EtOAc 2:1. The corresponding 4faa was obtained as a white solid $(68.9 \mathrm{mg}$, $41 \%$ yield). m.p. $=197.6-198.1 \stackrel{\circ}{\circ}$. ${ }^{1} \mathrm{H}$ NMR $\left(\mathrm{CDCl}_{3}, 400 \mathrm{MHz}\right): \delta 8.85$ (br s, $\left.1 \mathrm{H}\right), 7.78$ (d, $J=2.0 \mathrm{~Hz}, 1 \mathrm{H}), 7.38(\mathrm{~m}, 2 \mathrm{H}), 6.69(\mathrm{~d}, \mathrm{~J}=8.3 \mathrm{~Hz}, 1 \mathrm{H}), 3.62(\mathrm{~m}, 1 \mathrm{H}), 3.43(\mathrm{~m}, 1 \mathrm{H}), 2.97(\mathrm{~m}$, $2 \mathrm{H}), 1.37(\mathrm{~s}, 9 \mathrm{H}) \cdot{ }^{13} \mathrm{C} \mathrm{NMR}\left(\mathrm{CDCl}_{3}, 100 \mathrm{MHz}\right): \delta 172.2,167.2,161.0,139.8,133.0,129.6$, 127.8, 116.1, 112.4, 67.6, 52.4, 39.6, 35.9, 28.4 (3C). HRMS (ESI) calculated for $\mathrm{C}_{16} \mathrm{H}_{19} \mathrm{BrN}_{3} \mathrm{O}_{3}[\mathrm{MH}]^{+} 380.0604$, found 360.0605 .

N-(tert-Butyl)-5-iodo-2-oxo-3-(2-oxoazetidin-1-yl)indoline-3-carboxamide (4gaa):

Prepared using 5-iodoisatin, 3-aminopropanoic acid and tert-butyl isocyanide. CC: Hexane:EtOAc 2:1. The corresponding 4gaa was obtained as a white solid $(75.5 \mathrm{mg}$, $48 \%$ yield). m.p. $=204.0-205.3 \stackrel{\circ}{\circ} \mathrm{C} .{ }^{1} \mathrm{H}$ NMR $\left(\mathrm{CDCl}_{3}, 400 \mathrm{MHz}\right): \delta 8.58$ (br s, $\left.1 \mathrm{H}\right), 7.94$ (d, $J=1.7 \mathrm{~Hz}, 1 \mathrm{H}), 7.58$ (dd, J=8.2 Hz, 1.8, 1H), 7.35 (br s, $1 \mathrm{H}), 6.60$ (d, J=8.2 Hz, 1H), 3.62$3.59(\mathrm{~m}, 1 \mathrm{H}), 3.43-3.40(\mathrm{~m}, 1 \mathrm{H}), 3.03-2.91(\mathrm{~m}, 2 \mathrm{H}), 1.37(\mathrm{~s}, 9 \mathrm{H}) \cdot{ }^{13} \mathrm{C} \mathrm{NMR}\left(\mathrm{CDCl}_{3}, 100\right.$ $\mathrm{MHz}): \delta 172.0,167.0,161.0,140.3,139.0,135.2,128.0,112.8,86.1,67.4,52.4,39.6$, 36.0, 28.4 (3C). HRMS (ESI) calculated for $\mathrm{C}_{16} \mathrm{H}_{19} \mathrm{IN}_{3} \mathrm{O}_{3}[\mathrm{MH}]^{+}$428.0466, found 428.0463; and calculated for $\mathrm{C}_{16} \mathrm{H}_{18} \mathrm{IN}_{3} \mathrm{NaO}_{3}[\mathrm{MNa}]^{+} 450.0285$, found 450.0283 .

N-(tert-Butyl)-5-nitro-2-oxo-3-(2-oxoazetidin-1-yl)indoline-3-carboxamide (4haa):

Prepared using 5-nitroisatin, 3-aminopropanoic acid and tert-butyl isocyanide. CC: Hexane:EtOAc 2:1. The corresponding 4haa was obtained as a white solid $(52.0 \mathrm{mg}$, $29 \%$ yield). m.p.= 199.2-200.7 ํ․ ${ }^{1} \mathrm{H}$ NMR $\left(\left(\mathrm{CD}_{3}\right)_{2} \mathrm{CO}, 400 \mathrm{MHz}\right): \delta 8.50$ (d, J=2.4 Hz, 
$1 \mathrm{H}), 8.29$ (dd, J=8.7, 2.4 Hz, 1H), 7.37 (br s, $1 \mathrm{H}), 7.20(\mathrm{~d}, J=8.7,1 \mathrm{H}), 3.64-3.60(\mathrm{~m}, 1 \mathrm{H})$, 3.56-3.52 (m, $1 \mathrm{H}), 2.95-2.92(\mathrm{~m}, 2 \mathrm{H}), 1.38(\mathrm{~s}, 9 \mathrm{H}) .{ }^{13} \mathrm{C} \mathrm{NMR}\left(\left(\mathrm{CD}_{3}\right)_{2} \mathrm{CO}, 100 \mathrm{MHz}\right): \delta$ 175.1, 169.3, 164.0, 150.6, 145.6, 129.9, 1247, 124.5, 112.7, 70.7, 54.2, 41.3, 38.3, 30.1 (3C). HRMS (ESI) calculated for $\mathrm{C}_{16} \mathrm{H}_{19} \mathrm{~N}_{4} \mathrm{O}_{5}[\mathrm{MH}]^{+} 347.1350$, found 347.1343; and calculated for $\mathrm{C}_{16} \mathrm{H}_{18} \mathrm{~N}_{4} \mathrm{NaO}_{5}[\mathrm{MNa}]^{+}$369.1169, found 369.1159.

\section{N-(tert-Butyl)-5, 7-dimethyl-2-oxo-3-(2-oxoazetidin-1-yl)indoline-3-carboxamide} (4iaa):

Prepared using 5,7-dimethylisatin, 3-aminopropanoic acid and tert-butyl isocyanide. CC: Hexane:EtOAc 1:1. The corresponding 4iaa was obtained as a white solid (161.7 mg, 86\% yield). m.p.= 179.8-181.1 ํㅡ. ${ }^{1} \mathrm{H}$ NMR $\left(\mathrm{CDCl}_{3}, 400 \mathrm{MHz}\right): \delta 8.37$ (br s, 1H), $7.38(\mathrm{br} \mathrm{s}, 1 \mathrm{H}), 7.30(\mathrm{~s}, 1 \mathrm{H}), 6.90(\mathrm{~s}, 1 \mathrm{H}), 3.57-3.56(\mathrm{~m}, 1 \mathrm{H}), 3.37-3.35(\mathrm{~m}$, $1 \mathrm{H}), 2.94-2.92(\mathrm{~m}, 2 \mathrm{H}), 2.31(\mathrm{~s}, 3 \mathrm{H}), 2.12(\mathrm{~s}, 3 \mathrm{H}), 1.37(\mathrm{~s}, 9 \mathrm{H}) \cdot{ }^{13} \mathrm{C} \mathrm{NMR}\left(\mathrm{CDCl}_{3}, 100\right.$ $\mathrm{MHz}): \delta$ 173.0, 167.1, 162.1, 136.7, 133.0, 132.1, 125.8, 1247, 119.4, 68.8, 52.2, 39.3, $35.9,28.5,21.1,16.2$. HRMS (ESI) calculated for $\mathrm{C}_{18} \mathrm{H}_{23} \mathrm{~N}_{3} \mathrm{NaO}_{3}[\mathrm{MNa}]^{+} 352.1632$, found 352.1624 .

$\mathrm{N}$-(tert-Butyl)-2-oxo-3-(2-oxoazetidin-1-yl)-7-(trifluoromethyl)indoline-3carboxamide (4jaa):

Prepared using 7-trifluoromethylisatin, 3-aminopropanoic acid and tert-butyl isocyanide. CC: Hexane:EtOAc 2:1. The corresponding 4jaa was obtained as a white solid (80.4 mg, 47\% yield). m.p. = 106.0-107.3 ํ․ ${ }^{1} \mathrm{H} \mathrm{NMR}\left(\mathrm{CDCl}_{3}, 400 \mathrm{MHz}\right)$ : $\delta 8.13$ (br s, $1 \mathrm{H}), 7.88(\mathrm{~d}, J=8 \mathrm{~Hz}, 1 \mathrm{H}), 7.52(\mathrm{~d}, J=8 \mathrm{~Hz}, 1 \mathrm{H}), 7.23$ (t, J=8 Hz, 1H), 7.18 (br s, 1H), 3.57-3.54 (m, 1H), 3.40-3.38 (m, 1H), $2.96(\mathrm{t}, \mathrm{J}=4 \mathrm{~Hz}, 2 \mathrm{H}), 1.38(\mathrm{~s}, 9 \mathrm{H}) .{ }^{13} \mathrm{C} \mathrm{NMR}\left(\mathrm{CDCl}_{3}\right.$, $100 \mathrm{MHz}): \delta 172.4,166.9,160.8,137.6,130.8,127.3,127.0,126.9,123.5,113.0,112.6$, 67.1, 52.5, 39.4, 36.2, 28.4 (3C). HRMS (ESI) calculated for $\mathrm{C}_{17} \mathrm{H}_{18} \mathrm{~F}_{3} \mathrm{~N}_{3} \mathrm{NaO}_{3}[\mathrm{MNa}]^{+}$ 392.1192 , found 392.1183 .

N-Cyclohexyl-2-oxo-3-(2-oxoazetidin-1-yl)indoline-3-carboxamide (4aba):

Prepared using isatin, 3-aminopropanoic acid and cyclohexyl isocyanide. CC: Hexane:EtOAc 2:1. The corresponding 4aba was obtained as a pale yellow solid (152.7 mg, 69\% yield). m.p.= 187.8-189.1 으. ${ }^{1} \mathrm{H} \mathrm{NMR}\left(\mathrm{CDCl}_{3}, 400 \mathrm{MHz}\right): \delta 8.65$ (br s, $\left.1 \mathrm{H}\right), 7.66$ (d, J=8 Hz, 1H), 7.35 (d, J=4 Hz, 1H), 7.26 (t, J=8 Hz, 1H), 7.09 (t, J=8 Hz, 1H), 6.83 (d, $J=8 \mathrm{~Hz}, 1 \mathrm{H}), 3.79-3.74(\mathrm{~m}, 1 \mathrm{H}), 3.62-3.58(\mathrm{~m}, 1 \mathrm{H}), 3.40-3.36(\mathrm{~m}, 1 \mathrm{H}), 2.94-2.92(\mathrm{~m}, 1 \mathrm{H})$, 1.96-1.16 (m, 11H). ${ }^{13} \mathrm{C} \mathrm{NMR}\left(\mathrm{CDCl}_{3}, 100 \mathrm{MHz}\right): \delta 172.4,167.5,162.6,140.8,130.2$, 126.5, 126.0, 123.4, 110.8, 68.1, 49.1, 39.5, 35.9, 32.6, 32.4, 25.4, 24.6. HRMS (ESI) calculated for $\mathrm{C}_{18} \mathrm{H}_{21} \mathrm{~N}_{3} \mathrm{NaO}_{3}[\mathrm{MNa}]^{+} 350.1475$, found 350.1467 . 
2-Oxo-3-(2-oxoazetidin-1-yl)-N-(2,4,4-trimethylpentan-2-yl)indoline-3carboxamide (4aca):

Prepared using isatin, 3-aminopropanoic acid and tert-octyl isocyanide. CC: Hexane:EtOAc 1.5:1. The corresponding 4aca was obtained as a white solid (170.7 mg, $70 \%$ yield). m.p.= 139.8-140.7 ํㅡ. ${ }^{1} \mathrm{H}$ NMR $\left(\mathrm{CDCl}_{3}, 400 \mathrm{MHz}\right): \delta 8.51$ (br s, 1H), 7.67 (d, $J=8 \mathrm{~Hz}, 1 \mathrm{H}$ ), 7.26 (td, J=8, $4 \mathrm{~Hz}, 1 \mathrm{H}), 7.15$ (br s, 1H), 7.09 (td, J=7.6, $0.9 \mathrm{~Hz}, 1 \mathrm{H}), 6.82$ $(\mathrm{d}, \mathrm{J}=8 \mathrm{~Hz}, 1 \mathrm{H}), 3.65-3.61(\mathrm{~m}, 1 \mathrm{H}), 3.44-3.40(\mathrm{~m}, 1 \mathrm{H}), 2.95-2.92(\mathrm{~m}, 2 \mathrm{H}), 1.83(\mathrm{~d}, \mathrm{~J}=15$ $\mathrm{Hz}, 1 \mathrm{H}), 1.56$ (d, J=15 Hz, $1 \mathrm{H}), 1.44(\mathrm{~s}, 3 \mathrm{H}), 1.43(\mathrm{~s}, 3 \mathrm{H}), 0.94(9 \mathrm{H}) .{ }^{13} \mathrm{C} \mathrm{NMR}\left(\mathrm{CDCl}_{3}\right.$, $100 \mathrm{MHz}): \delta 172.4,167.3,161.8,140.7,130.1,126.7,126.0,123.4,110.7,68.5,56.3$, 52.1, 39.6, 36.0, 31.6, 31.3 (3C), 28.6, 28.5. HRMS (ESI) calculated for $\mathrm{C}_{20} \mathrm{H}_{27} \mathrm{~N}_{3} \mathrm{NaO}_{3}$ $[\mathrm{MNa}]^{+} 380.1945$, found 380.1935 .

N-Benzyl-2-oxo-3-(2-oxoazetidin-1-yl)indoline-3-carboxamide (4ada):

Prepared using isatin, 3-aminopropanoic acid and benzyl isocyanide. CC: Hexane:EtOAc 1.5:1. The corresponding 4ada was obtained as a white solid (131.2 mg, $58 \%$ yield). m.p. $=172.2-173.0 \stackrel{\circ}{\circ}$. ${ }^{1} \mathrm{H}$ NMR $\left(\mathrm{CDCl}_{3}, 400 \mathrm{MHz}\right): \delta 8.32$ (br s, $\left.1 \mathrm{H}\right), 7.69-$ $7.66(\mathrm{~m}, 2 \mathrm{H}), 7.34-7.26(\mathrm{~m}, 6 \mathrm{H}), 7.10(\mathrm{t}, \mathrm{J}=8 \mathrm{~Hz}, 1 \mathrm{H}), 6.84(\mathrm{~d}, \mathrm{~J}=8 \mathrm{~Hz}, 1 \mathrm{H}), 4.56-4.39(\mathrm{~m}$, $2 \mathrm{H}), 3.58-3.54(\mathrm{~m}, 1 \mathrm{H}), 3.34-3.30(\mathrm{~m}, 1 \mathrm{H}), 2.92(\mathrm{t}, \mathrm{J}=4 \mathrm{~Hz}, 2 \mathrm{H}) \cdot{ }^{13} \mathrm{C} \mathrm{NMR}\left(\mathrm{CDCl}_{3}, 100\right.$ $\mathrm{MHz}): \delta 172.0,167.6,163.7,140.6,137.3,130.4,128.8(2 \mathrm{C}), 127.8(2 \mathrm{C}), 127.7,126.7$, 125.8, 123.6, 110.8, 68.2, 44.2, 39.5, 36.1. HRMS (ESI) calculated for $\mathrm{C}_{19} \mathrm{H}_{17} \mathrm{~N}_{3} \mathrm{NaO}_{3}$ $[\mathrm{MNa}]^{+}$358.1162, found 358.1153.

1-Benzyl-2-oxo-3-(2-oxoazetidin-1-yl)-N-(2,4,4-trimethylpentan-2-yl)indoline-3carboxamide (4eca):

Prepared using $\mathrm{N}$-benzylisatin, 3-aminopropanoic acid and tert-octyl isocyanide. CC: Hexane:EtOAc 1.5:1. The corresponding 4eca was obtained as a white solid (80.6 mg, 43\% yield). m.p.= 113.1-113.9 $\stackrel{\circ}{ } \mathrm{C} .{ }^{1} \mathrm{H}$ NMR $\left(\mathrm{CDCl}_{3}, 400 \mathrm{MHz}\right): \delta 7.73$ (d, J=8 Hz, 1H), 7.35-7.21 (m, 6H), 7.12-7.08 (m, 2H), $6.73(\mathrm{~d}, J=8 \mathrm{~Hz}, 1 \mathrm{H}), 4.98(\mathrm{~d}, J=16,1 \mathrm{H}), 4.92$ (d, J=16, 1H), 3.63-3.60 (m, 1H), 3.39-3.36 (m, 1H), 2.93 (t, J=4 Hz, 2H), 1.83 (d, J=16 $\mathrm{Hz}, 1 \mathrm{H}), 1.53(\mathrm{~d}, \mathrm{~J}=16 \mathrm{~Hz}, 1 \mathrm{H}), 1.44(\mathrm{~s}, 3 \mathrm{H}), 1.43(\mathrm{~s}, 3 \mathrm{H}), 0.92$ (s, 9H). Compound previously described in the literature. ${ }^{1}$

N-Benzyl-2-oxo-3-(2-oxoazetidin-1-yl)-1-(prop-2-yn-1-yl)indoline-3-carboxamide (4cda):

Prepared using $\mathrm{N}$-propargylisatin, 3-aminopropanoic acid and benzyl isocyanide. CC: Hexane:EtOAc 2:1. The corresponding 4cda was obtained as a beige solid (82.7 
mg, $41 \%$ yield). m.p.= 167.3-168.1 ํ․ ${ }^{1} \mathrm{H}$ NMR $\left(\mathrm{CDCl}_{3}, 400 \mathrm{MHz}\right): \delta 7.76$ (d, J=8 Hz, $1 \mathrm{H}), 7.58$ (br s, $1 \mathrm{H}), 7.40$ (td, J=8, $4 \mathrm{~Hz}, 1 \mathrm{H}), 7.35-7.26(\mathrm{~m}, 5 \mathrm{H}), 7.18$ (td, J=7.6, $0.8 \mathrm{~Hz}$, $1 \mathrm{H}), 7.08(\mathrm{~d}, \mathrm{~J}=8 \mathrm{~Hz}, 1 \mathrm{H}), 4.58-4.39(\mathrm{~m}, 4 \mathrm{H}), 3.53-3.49(\mathrm{~m}, 1 \mathrm{H}), 3.27-3.24(\mathrm{~m}, 1 \mathrm{H}), 2.93-$ $2.90(\mathrm{~m}, 2 \mathrm{H}), 2.25$ (t, J=2.5, 1H). ${ }^{13} \mathrm{C} \mathrm{NMR}\left(\mathrm{CDCl}_{3}, 100 \mathrm{MHz}\right): \delta 170.0,167.5,163.6$, 141.6, 137.3, 130.4, 128.8 (2C), 127.8 (2C), 127.6, 126.8, 125.1, 124.1, 109.9, 76.0, 73.0, 68.1, 44.2, 39.2, 36.2, 29.9. HRMS (ESI) calculated for $\mathrm{C}_{22} \mathrm{H}_{19} \mathrm{~N}_{3} \mathrm{NaO}_{3}$ [MNa] ${ }^{+}$ 396.1319 , found 396.1311 .

N-Cyclohexyl-1-methyl-2-oxo-3-(2-oxoazetidin-1-yl)indoline-3-carboxamide (4bba):

Prepared using $\mathrm{N}$-methylisatin, 3-aminopropanoic acid and cyclohexyl isocyanide. CC: Hexane:EtOAc 1.5:1. The corresponding 4bba was obtained as a white solid (141.6 mg, 67\% yield). m.p.= 158.2-159.1 $\stackrel{\circ}{ } \mathrm{C} .{ }^{1} \mathrm{H} \mathrm{NMR}\left(\mathrm{CDCl}_{3}, 400 \mathrm{MHz}\right): \delta 7.73$ (d, J=8 Hz, $1 \mathrm{H}), 7.39-7.27$ (m, 2H), 7.14 (td, J=7.6, $0.9 \mathrm{~Hz}, 1 \mathrm{H}), 6.86$ (d, J=8 Hz, 1H), 3.79-3.73 (m, $1 \mathrm{H}), 3.54-3.49(\mathrm{~m}, 1 \mathrm{H}), 3.34-3.30(\mathrm{~m}, 1 \mathrm{H}), 3.26(\mathrm{~s}, 3 \mathrm{H}), 2.90(\mathrm{t}, J=4.3,2 \mathrm{H}), 1.97-1.94$ $(\mathrm{m}, 1 \mathrm{H}), 1.85-1.81(\mathrm{~m}, 1 \mathrm{H}), 1.74-1.66(\mathrm{~m}, 2 \mathrm{H}), 1.61-1.56(\mathrm{~m}, 1 \mathrm{H}), 1.39-1.19(\mathrm{~m}, 5 \mathrm{H}) .{ }^{13} \mathrm{C}$ $\mathrm{NMR}\left(\mathrm{CDCl}_{3}, 100 \mathrm{MHz}\right): \delta 171.2,167.1,162.4,143.3,130.2,126.7,125.3,123.7,108.8$, 67.7, 49.1, 39.2, 16.0, 32.6, 32.4, 26.8, 25.4, 24.6. HRMS (ESI) calculated for $\mathrm{C}_{19} \mathrm{H}_{23} \mathrm{~N}_{3} \mathrm{NaO}_{3}[\mathrm{MNa}]^{+}$364.1632, found 364.1622.

1-Methyl-2-oxo-3-(2-oxoazetidin-1-yl)-N-(2,4,4-trimethylpentan-2-yl)indoline-3carboxamide (4bca):

Prepared using $\mathrm{N}$-methylisatin, 3-aminopropanoic acid and tert-octyl isocyanide. CC: Hexane:EtOAc 1.5:1. The corresponding 4bca was obtained as a white solid (114.2 mg, $50 \%$ yield). m.p. $=107.2-108.5 \stackrel{\circ}{ } \mathrm{C} .{ }^{1} \mathrm{H} \mathrm{NMR}\left(\mathrm{CDCl}_{3}, 400 \mathrm{MHz}\right): \delta 7.73$ (d, J=8 Hz, $1 \mathrm{H}$ ), 7.36 (td, J=7.7, $1.2 \mathrm{~Hz}, 1 \mathrm{H}), 7.16-7.12(\mathrm{~m}, 2 \mathrm{H}), 6.86$ (d, J=8 Hz, 1H), 3.58-3.55 (m, 1H), 3.36-3.32 (m, 1H), 3.25 (s, 3H), 2.90 (t, J=4.3 Hz, 2H), 1.80 (d, J=15 Hz, 1H), 1.55

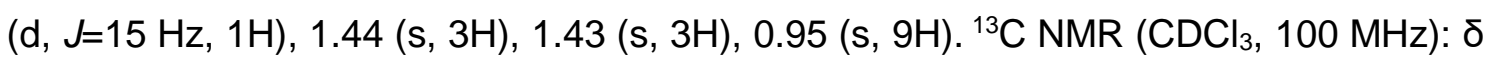
171.2, 167.0, 161.8, 143.2, 130.1, 126.8, 125.5, 123.6, 108.7, 68.4, 56.2, 52.2, 39.3, 36.0, 31.6, 31.3 (3C), 28.6, 28.4, 26.7. HRMS (ESI) calculated for $\mathrm{C}_{21} \mathrm{H}_{29} \mathrm{~N}_{3} \mathrm{NaO}_{3}[\mathrm{MNa}]^{+}$ 394.2101 , found 394.2092 .

\subsection{General procedure for the synthesis of $\mathrm{V}$-lactam-oxindole hybrids} obtained via U4c3CR (library 5). To a stirring solution of isatin derivative (100 mg, 1.0 equiv) and 4-aminobutanoic acid ( 1.0 equiv) in TFE ( $1 \mathrm{~mL}$ ), isocyanide (1.0 equiv) was added. The reaction was allowed to stir at room temperature and the progress monitored by TLC. Upon completion, or when no change was detected, the magnetic stir bar was 
removed, and the solvent evaporated under reduced pressure. The crude reaction mixture was purified by column chromatography (CC). The structural elucidation and purity evaluation was performed using NMR, HRMS, ATR-FTIR and melting point determination.

\section{$N$-(tert-Butyl)-2-oxo-3-(2-oxopyrrolidin-1-yl)indoline-3-carboxamide (5aab):}

Prepared using isatin, 4-aminobutanoic acid and tert-butyl isocyanide. CC: Hexane:EtOAc 2:1. The corresponding 5aab was obtained as a pale yellow solid (71.6 mg, 33\% yield). m.p. $=235.0^{\circ} \mathrm{C}$ (decomp.). ${ }^{1} \mathrm{H}$ NMR $\left(\mathrm{CDCl}_{3}, 400 \mathrm{MHz}\right): \delta 8.51$ (br s, $\left.1 \mathrm{H}\right)$, $7.50(\mathrm{~d}, J=7.0 \mathrm{~Hz}, 1 \mathrm{H}), 7.34(\mathrm{br} \mathrm{s}, 1 \mathrm{H}), 7.22$ (td, J=7.7, $1.0 \mathrm{~Hz}, 1 \mathrm{H}), 7.06$ (td, J=7.6, 0.7 $\mathrm{Hz}, 1 \mathrm{H}), 6.77(\mathrm{~d}, J=7.8 \mathrm{~Hz}, 1 \mathrm{H}), 3.87-3.81(\mathrm{~m}, 1 \mathrm{H}), 3.70-3.64(\mathrm{~m}, 1 \mathrm{H}), 2.48-2.40(\mathrm{~m}, 1 \mathrm{H})$, 2.34-2.26 (m, 1H), 2.19-2.12 (m, 2H), $1.35(\mathrm{~s}, 9 \mathrm{H}) .{ }^{13} \mathrm{C} \mathrm{NMR}\left(\mathrm{CDCl}_{3}, 100 \mathrm{MHz}\right): \delta 175.1$, 174.8, 161.6, 141.2, 129.6, 126.4, 126.2, 123.1, 110.5, 67.7, 52.1, 46.7, 30.7, 28.4 (3C), 18.8. HRMS (ESI) calculated for $\mathrm{C}_{17} \mathrm{H}_{22} \mathrm{~N}_{3} \mathrm{O}_{3}[\mathrm{MH}]^{+}$316.1656, found 316.1659; and calculated for $\mathrm{C}_{17} \mathrm{H}_{21} \mathrm{~N}_{3} \mathrm{NaO}_{3}[\mathrm{MNa}]^{+} 338.1475$, found 338.1477.

\section{N-(tert-Butyl)-1-methyl-2-oxo-3-(2-oxopyrrolidin-1-yl)indoline-3-carboxamide} (5bab):

Prepared using $\mathrm{N}$-methylisatin, 4-aminobutanoic acid and tert-butyl isocyanide. CC: Hexane:EtOAc 2:1. The corresponding 5 bab was obtained as a white solid (52.9 mg, $26 \%$ yield). m.p.=179.2-180.1 ํ․ ${ }^{1} \mathrm{H}$ NMR $\left(\mathrm{CDCl}_{3}, 400 \mathrm{MHz}\right): \delta 7.55$ (d, J=7.4 Hz, $1 \mathrm{H}), 7.35-7.31(\mathrm{~m}, 2 \mathrm{H}), 7.11(\mathrm{t}, J=7.5 \mathrm{~Hz}, 1 \mathrm{H}), 6.85(\mathrm{~d}, J=7.8 \mathrm{~Hz}, 1 \mathrm{H}), 3.81-3.75(\mathrm{~m}, 1 \mathrm{H})$, 3.65-3.59 (m, $1 \mathrm{H}), 3.28(\mathrm{~s}, 3 \mathrm{H}), 2.41-2.33(\mathrm{~m}, 1 \mathrm{H}), 2.28-2.19(\mathrm{~m}, 1 \mathrm{H}), 2.17-2.07(\mathrm{~m}, 2 \mathrm{H})$, 1.37 (s, 9H). ${ }^{13} \mathrm{C} \mathrm{NMR}\left(\mathrm{CDCl}_{3}, 100 \mathrm{MHz}\right): \delta 174.9,173.5,161.9,143.9,129.7,1263$, 125.8, 123.3, 108.4, 67.4, 52.1, 46.4, 30.4, 28.4 (3C), 27.0, 18.8. HRMS (ESI) calculated for $\mathrm{C}_{18} \mathrm{H}_{23} \mathrm{~N}_{3} \mathrm{NaO}_{3}[\mathrm{MNa}]^{+}$352.1632, found 352.1622.

\section{N-(tert-Butyl)-2-oxo-3-(2-oxopyrrolidin-1-yl)-1-phenylindoline-3-carboxamide} (5dab):

Prepared using $\mathrm{N}$-phenylisatin, 4-aminobutanoic acid and tert-butyl isocyanide. CC: Hexane:EtOAc 2:1. The corresponding 5dab was obtained as a beige solid (52.3 mg, 30\% yield). m.p.= 185.7-186.3 ${ }^{\circ} \mathrm{C} .{ }^{1} \mathrm{H}$ NMR $\left(\mathrm{CDCl}_{3}, 400 \mathrm{MHz}\right): \delta 7.61$ (dd, $\mathrm{J}=7.4,0.9$ $\mathrm{Hz}, 1 \mathrm{H}), 7.56-7.41(\mathrm{~m}, 6 \mathrm{H}), 7.24(\mathrm{td}, J=7.8,1.3 \mathrm{~Hz}, 1 \mathrm{H}), 7.13(\mathrm{td}, J=7.6,1.0 \mathrm{~Hz}, 1 \mathrm{H})$, $6.75(\mathrm{~d}, J=7.8 \mathrm{~Hz}, 1 \mathrm{H}), 3.89-3.83(\mathrm{~m}, 1 \mathrm{H}), 3.75-3.69(\mathrm{~m}, 1 \mathrm{H}), 2.44-2.40(\mathrm{~m}, 1 \mathrm{H}), 2.32-$ $2.16(\mathrm{~m}, 3 \mathrm{H}), 1.38(\mathrm{~s}, 9 \mathrm{H}) .{ }^{13} \mathrm{C}$ NMR $\left(\mathrm{CDCl}_{3}, 100 \mathrm{MHz}\right): \delta$ 175.0, 173.2, 161.7, 144.3, 134.4, 129.7 (2C), 129.6, 128.5, 126.9 (2C), 126.6, 125.3, 123.6, 109.6, 67.3, 52.2, 46.4, 
30.4, 28.4 (3C), 19.0. HRMS (ESI) calculated for $\mathrm{C}_{23} \mathrm{H}_{25} \mathrm{~N}_{3} \mathrm{NaO}_{3}[\mathrm{MNa}]^{+} 414.1788$, found 414.1779 .

1-Benzyl-N-(tert-butyl)-2-oxo-3-(2-oxopyrrolidin-1-yl)indoline-3-carboxamide (5eab):

Prepared using $\mathrm{N}$-benzylisatin, 4-aminobutanoic acid and tert-butyl isocyanide. CC: Hexane:EtOAc 1.5:1. The corresponding 5eab was obtained as a white solid (81.4 mg, 48\% yield). m.p.= 201.1-202.3 $\stackrel{\circ}{ } \mathrm{C} .{ }^{1} \mathrm{H}$ NMR $\left(\mathrm{CDCl}_{3}, 400 \mathrm{MHz}\right): \delta 7.54$ (dd, J=7.4, 0.8 $\mathrm{Hz}, 1 \mathrm{H}), 7.37-7.23(\mathrm{~m}, 6 \mathrm{H}), 7.18$ (td, J=7.8, $1.2 \mathrm{~Hz}, 1 \mathrm{H}), 7.06$ (td, J=7.6, $0.8 \mathrm{~Hz}, 1 \mathrm{H})$, $6.66(\mathrm{~d}, \mathrm{~J}=7.8 \mathrm{~Hz}, 1 \mathrm{H}), 4.98(\mathrm{~s}, 2 \mathrm{H}), 3.85-3.79(\mathrm{~m}, 1 \mathrm{H}), 3.68-3.62(\mathrm{~m}, 1 \mathrm{H}), 2.47-2.39(\mathrm{~m}$, $1 \mathrm{H}), 2.34-2.25(\mathrm{~m}, 1 \mathrm{H}), 2.19-2.10(\mathrm{~m}, 2 \mathrm{H}), 1.37(\mathrm{~s}, 9 \mathrm{H}) .{ }^{13} \mathrm{C} \mathrm{NMR}\left(\mathrm{CDCl}_{3}, 100 \mathrm{MHz}\right): \delta$ 174.9, 173.6, 161.8, 142.7, 135.3, 129.5, 128.8 (2C), 1276, 127.1 (2C), 126.3, 125.9, 123.4, 109.5, 67.9, 52.1, 46.6, 44.5, 30.5, 28.4 (3C), 18.9. HRMS (ESI) calculated for $\mathrm{C}_{24} \mathrm{H}_{27} \mathrm{~N}_{3} \mathrm{NaO}_{3}[\mathrm{MNa}]^{+}$428.1945, found 428.1936.

\section{5-Bromo-N-(tert-butyl)-2-oxo-3-(2-oxopyrrolidin-1-yl)indoline-3-carboxamide} (5fab):

Prepared using 5-bromoisatin, 4-aminobutanoic acid and tert-butyl isocyanide. CC: Hexane:EtOAc 1.5:1. The corresponding $5 \mathrm{fab}$ was obtained as a beige solid $(38.0 \mathrm{mg}$, $22 \%$ yield). m.p.= 240.1-241.3 ${ }^{\circ} \mathrm{C} .{ }^{1} \mathrm{H}$ NMR $\left(\mathrm{CDCl}_{3}, 400 \mathrm{MHz}\right): \delta 8.40$ (br s, 1H), 7.62 (d, $J=1.8 \mathrm{~Hz}, 1 \mathrm{H}), 7.35$ (dd, J=8.3, $2.0 \mathrm{~Hz}, 1 \mathrm{H}), 7.33$ (br s, 1H), 6.67 (d, J=8.3 Hz, 1H), 3.84$3.78(\mathrm{~m}, 1 \mathrm{H}), 3.70-3.64(\mathrm{~m}, 1 \mathrm{H}), 2.49-2.41(\mathrm{~m}, 1 \mathrm{H}), 2.37-2.28(\mathrm{~m}, 1 \mathrm{H}), 2.21-2.14(\mathrm{~m}, 2 \mathrm{H})$, $1.36(\mathrm{~s}, 9 \mathrm{H}) .{ }^{13} \mathrm{C} \mathrm{NMR}\left(\mathrm{CDCl}_{3}, 100 \mathrm{MHz}\right): \delta 175.2,174.5,160.9,140.2,132.4,129.6$, 128.0, 115.8, 111.8, 67.5, 52.3, 46.6, 30.5, 28.4 (3C), 18.8. HRMS (ESI) calculated for $\mathrm{C}_{17} \mathrm{H}_{21} \mathrm{~N}_{3} \mathrm{O}_{3} \mathrm{Br}[\mathrm{MH}]^{+}$394.0761, found 394.0756; and calculated for $\mathrm{C}_{17} \mathrm{H}_{20} \mathrm{BrN}_{3} \mathrm{NaO}_{3}$ $[\mathrm{MNa}]^{+}$416.0580, found 416.0573.

N-(tert-Butyl)-5-iodo-2-oxo-3-(2-oxopyrrolidin-1-yl)indoline-3-carboxamide (5gab):

Prepared using 5-iodoisatin, 4-aminobutanoic acid and tert-butyl isocyanide. CC: Hexane:EtOAc 1.5:1. The corresponding $5 \mathrm{gab}$ was obtained as a white solid (34.8 $\mathrm{mg}$, $22 \%$ yield). m.p. $=239.5-241.0 \stackrel{\circ}{\circ} .{ }^{1} \mathrm{H}$ NMR $\left(\mathrm{CDCl}_{3}, 400 \mathrm{MHz}\right): \delta 8.19$ (br s, $\left.1 \mathrm{H}\right), 7.78$ (d, $J=1.6 \mathrm{~Hz}, 1 \mathrm{H}$ ), 7.55 (dd, $J=8.2,1.8 \mathrm{~Hz}, 1 \mathrm{H}$ ), 7.31 (br s, $1 \mathrm{H}), 6.58(\mathrm{~d}, J=8.2 \mathrm{~Hz}, 1 \mathrm{H}), 3.83$ $3.77(\mathrm{~m}, 1 \mathrm{H}), 3.68-3.63(\mathrm{~m}, 1 \mathrm{H}), 2.48-2.40(\mathrm{~m}, 1 \mathrm{H}), 2.36-2.28(\mathrm{~m}, 1 \mathrm{H}), 2.21-2.13(\mathrm{~m}, 2 \mathrm{H})$, $1.36(\mathrm{~s}, 9 \mathrm{H}) .{ }^{13} \mathrm{C} \mathrm{NMR}\left(\mathrm{CDCl}_{3}, 100 \mathrm{MHz}\right): \delta$ 175.2, 174.3, 160.9, 140.8, 138.4,135.2, 128.2, 112.3, 85.8, 67.4, 52.3, 46.6, 30.5, 28.4 (3C), 18.8. HRMS (ESI) calculated for $\mathrm{C}_{17} \mathrm{H}_{20} \mathrm{IN}_{3} \mathrm{NaO}_{3}[\mathrm{MNa}]^{+}$464.0442, found 464.0436. 
$\mathrm{N}$-(tert-Butyl)-5-nitro-2-oxo-3-(2-oxopyrrolidin-1-yl)indoline-3-carboxamide (5hab):

Prepared using 5-nitroisatin, 4-aminobutanoic acid and tert-butyl isocyanide. CC: Hexane:EtOAc 2:1. The corresponding 5 hab was obtained as a beige solid $(101.0 \mathrm{mg}$, $54 \%$ yield). m.p. $=218.5$ (decomp.). ${ }^{1} \mathrm{H} \mathrm{NMR}\left(\mathrm{CDCl}_{3}, 400 \mathrm{MHz}\right.$ ): $\delta 9.00$ (br s, $\left.1 \mathrm{H}\right), 8.38$ (d, J=2.2 Hz, $1 \mathrm{H}), 8.22-8.19(\mathrm{~m}, 1 \mathrm{H}), 7.26-7.23(\mathrm{~m}, 1 \mathrm{H}), 6.86(\mathrm{dd}, J=8.6,3.1 \mathrm{~Hz}, 1 \mathrm{H})$, 3.95-3.89 (m, 1H), 3.78-3.73 (m, 1H), 2.54-2.45 (m, 1H), 2.40-2.32 (m, 1H), 2.28-2.23

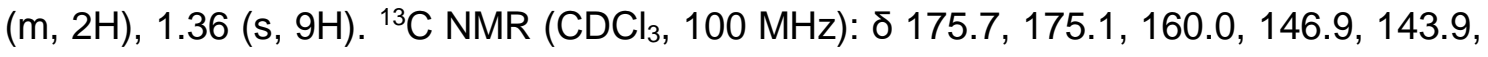
126.9, 126.4, 122.6, 110.3, 67.2, 52.6, 46.8, 30.4, 28.3 (3C), 18.8. HRMS (ESI) calculated for $\mathrm{C}_{17} \mathrm{H}_{20} \mathrm{~N}_{4} \mathrm{NaO}_{5}[\mathrm{MNa}]^{+} 383.1326$, found 383.1320 .

$N$-(tert-Butyl)-5,7-dimethyl-2-oxo-3-(2-oxopyrrolidin-1-yl)indoline-3-carboxamide (5iab):

Prepared using 5,7-dimethylisatin, 4-aminobutanoic acid and tert-butyl isocyanide. CC: Hexane:EtOAc 1.5:1. The corresponding 5iab was obtained as a beige solid (73.6 $\mathrm{mg}, 38 \%$ yield). m.p. $=231.4-232.7^{\circ} \mathrm{C}$. ${ }^{1} \mathrm{H} \mathrm{NMR}\left(\mathrm{CDCl}_{3}, 400 \mathrm{MHz}\right): \delta 7.87$ (br s, $\left.1 \mathrm{H}\right), 7.31$ (br s, $1 \mathrm{H}), 7.15(\mathrm{~s}, 1 \mathrm{H}), 6.88(\mathrm{~s}, 1 \mathrm{H}), 3.83-3.77(\mathrm{~m}, 1 \mathrm{H}), 3.65-3.60(\mathrm{~m}, 1 \mathrm{H}), 2.46-2.37(\mathrm{~m}$, $1 \mathrm{H}), 2.33-2.25(\mathrm{~m}, 1 \mathrm{H}), 2.29(\mathrm{~s}, 3 \mathrm{H}), 2.17-2.10(\mathrm{~m}, 2 \mathrm{H}), 2.14(\mathrm{~s}, 3 \mathrm{H}), 1.36(\mathrm{~s}, 9 \mathrm{H}) \cdot{ }^{13} \mathrm{C}$ $\mathrm{NMR}\left(\mathrm{CDCl}_{3}, 100 \mathrm{MHz}\right): \delta$ 175.0, 174.9, 161.9, 137.0, 132.6, 131.7, 125.9, 124.6, 118.7, $68.2,52.0,46.5,30.5,28.4$ (3C), 21.1, 18.8, 16.2. HRMS (ESI) calculated for $\mathrm{C}_{19} \mathrm{H}_{25} \mathrm{~N}_{3} \mathrm{NaO}_{3}[\mathrm{MNa}]^{+} 366.1788$, found 366.1781 .

$\mathrm{N}$-(tert-Butyl)-2-oxo-3-(2-oxopyrrolidin-1-yl)-7-(trifluoromethyl)indoline-3carboxamide (5jab):

Prepared using 7-trifluoromethylisatin, 4-aminobutanoic acid and tert-butyl isocyanide. CC: Hexane:EtOAc 2:1. The corresponding 5jab was obtained as a white solid (72.8 mg, 41\% yield). m.p. $=169.8-171.2{ }^{\circ} \mathrm{C} .{ }^{1} \mathrm{H} \mathrm{NMR}\left(\mathrm{CDCl}_{3}, 400 \mathrm{MHz}\right): \delta 7.95$ (br s, $1 \mathrm{H}), 7.68(\mathrm{~d}, J=7.5 \mathrm{~Hz}, 1 \mathrm{H}), 7.48(\mathrm{~d}, J=8.0 \mathrm{~Hz}, 1 \mathrm{H}), 7.20-7.17(\mathrm{~m}, 2 \mathrm{H}), 3.83-3.77(\mathrm{~m}$, $1 \mathrm{H}), 3.68-3.62(\mathrm{~m}, 1 \mathrm{H}), 2.46-2.38(\mathrm{~m}, 1 \mathrm{H}), 2.32-2.24(\mathrm{~m}, 1 \mathrm{H}), 2.21-2.12(\mathrm{~m}, 2 \mathrm{H}), 1.38(\mathrm{~s}$, 9H). ${ }^{13} \mathrm{C} \mathrm{NMR}\left(\mathrm{CDCl}_{3}, 100 \mathrm{MHz}\right): \delta 175.2,174.6,160.7,138.1,130.2,127.9,126.5$, 126.4, 123.1, 112.5, 112.2, 66.7, 52.4, 46.4, 30.2, 28.4 (3C), 19.0. HRMS (ESI) calculated for $\mathrm{C}_{18} \mathrm{H}_{20} \mathrm{~F}_{3} \mathrm{~N}_{3} \mathrm{NaO}_{3}[\mathrm{MNa}]^{+} 406.1349$, found 406.1340 .

\section{N-Cyclohexyl-2-oxo-3-(2-oxopyrrolidin-1-yl)indoline-3-carboxamide (5abb):}

Prepared using isatin, 4-aminobutanoic acid and cyclohexyl isocyanide. CC: Hexane:EtOAc 2:1. The corresponding 5abb was obtained as a white solid $(65.7 \mathrm{mg}$, $28 \%$ yield). m.p. $=214.7-215.8 \stackrel{\circ}{\circ} \mathrm{C} .{ }^{1} \mathrm{H} \mathrm{NMR}\left(\mathrm{CDCl}_{3}, 400 \mathrm{MHz}\right)$ : $\delta 8.17$ (br s, $\left.1 \mathrm{H}\right), 7.53$ (d, $J=7.4 \mathrm{~Hz}, 1 \mathrm{H}), 7.28-7.22(\mathrm{~m}, 2 \mathrm{H}), 7.07(\mathrm{t}, J=7.6 \mathrm{~Hz}, 1 \mathrm{H}), 6.80(\mathrm{~d}, J=7.8 \mathrm{~Hz}, 1 \mathrm{H}), 3.84-$ 
$3.72(\mathrm{~m}, 2 \mathrm{H}), 3.64-3.58(\mathrm{~m}, 1 \mathrm{H}), 2.45-2.38(\mathrm{~m}, 1 \mathrm{H}), 2.35-2.26(\mathrm{~m}, 1 \mathrm{H}), 2.17-2.09(\mathrm{~m}, 2 \mathrm{H})$,

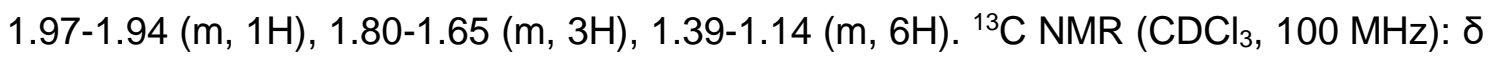
175.1, 174.6, 161.8, 141.0, 129.6, 126.5, 126.2, 123.2, 110.3, 67.3, 49.1, 46.8, 32.6, 32.3, 30.7, 25.4, 24.6, 24.5, 18.8. HRMS (ESI) calculated for $\mathrm{C}_{19} \mathrm{H}_{23} \mathrm{~N}_{3} \mathrm{NaO}_{3}[\mathrm{MNa}]^{+}$ 364.1632 , found 364.1623 .

2-Oxo-3-(2-oxopyrrolidin-1-yl)-N-(2,4,4-trimethylpentan-2-yl)indoline-3carboxamide (5acb):

Prepared using isatin, 4-aminobutanoic acid and tert-octyl isocyanide. CC: Hexane:EtOAc 1.5:1. The corresponding 5acb was obtained as a white solid (78.4 mg, $31 \%$ yield). m.p. $=198.5-199.2 \stackrel{\circ}{\circ} \mathrm{C} .{ }^{1} \mathrm{H}$ NMR $\left(\mathrm{CDCl}_{3}, 400 \mathrm{MHz}\right): \delta 8.35$ (br s, $\left.1 \mathrm{H}\right), 7.51$ (d, $J=7.4 \mathrm{~Hz}, 1 \mathrm{H}$ ), 7.25 (br s, 1H), 7.21 (td, J=7.7, $1.1 \mathrm{~Hz}, 1 \mathrm{H}), 7.06$ (td, $J=7.6,0.8 \mathrm{~Hz}, 1 \mathrm{H}$ ), $6.76(\mathrm{~d}, J=7.8 \mathrm{~Hz}, 1 \mathrm{H}), 3.86-3.80(\mathrm{~m}, 1 \mathrm{H}), 3.69-3.64(\mathrm{~m}, 1 \mathrm{H}), 2.47-2.38(\mathrm{~m}, 1 \mathrm{H}), 2.34-$ $2.26(\mathrm{~m}, 1 \mathrm{H}), 2.17-2.10(\mathrm{~m}, 2 \mathrm{H}), 1.89(\mathrm{~d}, \mathrm{~J}=14.9 \mathrm{~Hz}, 1 \mathrm{H}), 1.46-1.42(\mathrm{~m}, 7 \mathrm{H}), 0.93(\mathrm{~s}, 9 \mathrm{H})$. ${ }^{13} \mathrm{C} \mathrm{NMR}\left(\mathrm{CDCl}_{3}, 100 \mathrm{MHz}\right): \delta 175.1,174.6,161.2,141.0,129.5,126.4,126.3,123.1$, 110.4, 68.0, 56.1, 52.2, 46.7, 31.5, 31.3 (3C), 30.7, 28.6, 28.0, 18.9. HRMS (ESI) calculated for $\mathrm{C}_{21} \mathrm{H}_{29} \mathrm{~N}_{3} \mathrm{NaO}_{3}[\mathrm{MNa}]^{+} 394.2101$, found 394.2093.

\section{N-Benzyl-2-oxo-3-(2-oxopyrrolidin-1-yl)indoline-3-carboxamide (5adb):}

Prepared using isatin, 4-aminobutanoic acid and benzyl isocyanide. CC: Hexane:EtOAc 2:1. The corresponding 5adb was obtained as a white solid (48.7 $\mathrm{mg}$, $21 \%$ yield). m.p. $=215.8-217.1^{\circ} \mathrm{C} .{ }^{1} \mathrm{H}$ NMR $\left(\mathrm{CDCl}_{3}, 400 \mathrm{MHz}\right): \delta 8.08$ (br s, $\left.1 \mathrm{H}\right), 7.57-$ $7.56(\mathrm{~m}, 2 \mathrm{H}), 7.31-7.23(\mathrm{~m}, 6 \mathrm{H}), 7.08(\mathrm{td}, J=7.6,0.9 \mathrm{~Hz}, 1 \mathrm{H}), 6.81(\mathrm{~d}, J=7.8 \mathrm{~Hz}, 1 \mathrm{H}), 4.52$ (dd, J=14.7, $5.9 \mathrm{~Hz}, 1 \mathrm{H}$ ), 4.40 (dd, J=14.7, $5.7 \mathrm{~Hz}, 1 \mathrm{H}$ ), 3.76-3.70 (m, 1H), 3.47-3.42 (m, $1 \mathrm{H}), 2.44-2.26(\mathrm{~m}, 2 \mathrm{H}), 2.11-2.04(\mathrm{~m}, 2 \mathrm{H}) .{ }^{13} \mathrm{C} \mathrm{NMR}\left(\mathrm{CDCl}_{3}, 100 \mathrm{MHz}\right): \delta 175.3,174.1$, 163.2, 140.9, 137.3, 129.9, 128.8 (2C), 127.9 (2C), 127.7, 126.6, 126.0, 123.4, 110.4, 67.5, 46.7, 44.2, 30.6, 18.7. HRMS (ESI) calculated for $\mathrm{C}_{20} \mathrm{H}_{19} \mathrm{~N}_{3} \mathrm{NaO}_{3}[\mathrm{MNa}]^{+}$372.1319, found 372.1311 .

1-Methyl-2-oxo-3-(2-oxopyrrolidin-1-yl)-N-(2,4,4-trimethylpentan-2-yl)indoline-3carboxamide (5bcb):

Prepared using $\mathrm{N}$-methylisatin, 4-aminobutanoic acid and tert-octyl isocyanide. CC: Hexane:EtOAc 2:1. The corresponding 5 bcb was obtained as a beige solid (70.8 mg, 30\% yield). m.p.= 152.7-153.8 $\stackrel{\circ}{\mathrm{C} .}{ }^{1} \mathrm{H} \mathrm{NMR}\left(\mathrm{CDCl}_{3}, 400 \mathrm{MHz}\right): \delta 7.55$ (dd, J=7.4, 0.8 $\mathrm{Hz}, 1 \mathrm{H}$ ), 7.32 (td, J=7.8, 1.2 Hz, 1H), 7.29 (br s, 1H), 7.10 (td, J=7.6, $0.8 \mathrm{~Hz}, 1 \mathrm{H}$ ), 6.83 (d, J=7.8 Hz, 1H), 3.82-3.76 (m, 1H), 3.64-3.58 (m, 1H), $3.25(\mathrm{~s}, 3 \mathrm{H}), 2.41-2.33(\mathrm{~m}, 1 \mathrm{H})$, 2.29-2.20 (m, 1H), 2.15-2.09 (m, 2H), $1.89(\mathrm{~d}, \mathrm{~J}=15.0 \mathrm{~Hz}, 1 \mathrm{H}), 1.44-1.41(\mathrm{~m}, 7 \mathrm{H}), 0.92$ 
(s, 9H). ${ }^{13} \mathrm{C} \mathrm{NMR}\left(\mathrm{CDCl}_{3}, 100 \mathrm{MHz}\right): \delta 175.0,173.3,161.4,143.7,129.6,126.3,126.0$, $123.4,108.3,67.8,56.0,52.2,46.6,31.5,31.2$ (3C), 30.5, 28.6, 28.0, 26.8, 18.9. HRMS (ESI) calculated for $\mathrm{C}_{22} \mathrm{H}_{31} \mathrm{~N}_{3} \mathrm{NaO}_{3}[\mathrm{MNa}]^{+} 408.2258$, found 408.2249 .

N-Benzyl-2-oxo-3-(2-oxopyrrolidin-1-yl)-1-(prop-2-yn-1-yl)indoline-3-carboxamide (5cdb):

Prepared using $N$-propargylisatin, 4-aminobutanoic acid and benzyl isocyanide. CC: Hexane:EtOAc 2:1. The corresponding $5 \mathbf{c d b}$ was obtained as a beige solid (50.2 mg, 24\% yield). m.p.= $194.2{ }^{\circ} \mathrm{C}$ (decomp.). ${ }^{1} \mathrm{H} \mathrm{NMR}\left(\mathrm{CDCl}_{3}, 400 \mathrm{MHz}\right): \delta 7.62$ (dd, J=7.5, $0.7 \mathrm{~Hz}, 1 \mathrm{H}), 7.52$ (br s, 1H), 7.39-7.26 (m, 6H), 7.15 (td, J=7.6, $0.9 \mathrm{~Hz}, 1 \mathrm{H}), 7.07$ (d, $J=7.9 \mathrm{~Hz}, 1 \mathrm{H}$ ), 4.63 (dd, J=17.7, $2.6 \mathrm{~Hz}, 1 \mathrm{H}), 4.54$ (dd, $J=14.8,6.0 \mathrm{~Hz}, 1 \mathrm{H}$ ), 4.47 (dd, $J=17.7,2.5 \mathrm{~Hz}, 1 \mathrm{H}), 4.39$ (dd, $J=14.8,5.6 \mathrm{~Hz}, 1 \mathrm{H}), 3.72-3.67(\mathrm{~m}, 1 \mathrm{H}), 3.42-3.37(\mathrm{~m}, 1 \mathrm{H})$, 2.39-2.22 (m, 3H), 2.10-2.02 (m, 2H). ${ }^{13} \mathrm{C} \mathrm{NMR}\left(\mathrm{CDCl}_{3}, 100 \mathrm{MHz}\right): \delta$ 175.3, 172.0, 163.0, 142.0, 137.3, 129.9, 128.8 (2C), 127.9 (2C), 127.7, 126.5, 125.5, 123.9, 109.5, 76.2, 72.8, 67.3, 46.4, 44.2, 30.4, 30.1, 18.8. HRMS (ESI) calculated for $\mathrm{C}_{23} \mathrm{H}_{21} \mathrm{~N}_{3} \mathrm{NaO}_{3}[\mathrm{MNa}]^{+}$ 410.1475 , found 410.1469 .

1.3. Cholinesterase evaluation assay. Inhibition of AChE (electric eel) and BuChE (equine serum) was meaured using the Ellman's cholorimetric assay, ${ }^{3}$ with the minor modifications reported by us. ${ }^{4}$ DMSO (1.25\% final concentration in cuvette) was used for preparing the stock inhibitor solutions. Enzymatic kinetics were monitored by UV-Vis spectroscopy using DTNB (5,5'-dithiobis(2-nitrobenzoic acid), $0.975 \mathrm{mM}$ ) as the chromogenic agent. Reaction took place in a buffered medium ( $50 \mu \mathrm{M}$ phosphate buffer, $\mathrm{pH}$ 8.0), $\mathrm{T}=25^{\circ} \mathrm{C}$, being monitored for $125 \mathrm{~s}$ at $405 \mathrm{~nm}$.

For determining the percentage of inhibition, the substrate concentration (acetylthiocholine iodide for AChE; S-butyrylthiocholine iodide for BuChE) was fixed at $121 \mu \mathrm{M}$ for AChE and $112 \mu \mathrm{M}$ for BuChE. IC $_{50}$ values were calculated by plotting the percentage of inhibition vs. log[l] using 5-7 different inhibitor concentrations, and adjusting to a second-order equation. Cornish-Bowden plots (1/V vs. [I] and [S]/V vs. [I]) were used for estimating the mode of action, and the kinetic parameters ( $K_{\mathrm{M}}, K_{\mathrm{M} \text {,app }}, V_{\max }$, $\left.V_{\max , a p p}\right)$ were calculated using non-linear regression analysis (least squares fit) implemented in GraphPad Prism 8.01 software. Inhibition constants $\left(K_{i}\right)$ were calculated using the same equations as previously. ${ }^{4}$

1.4. Monoamine oxidases inhibition assay. Assessment of potential MAO A and $B$ inhibition was conducted. One-point screening of compounds were carried out for both isoforms using a discontinuous fluorimetric assay as described previously. ${ }^{5}$ In brief, MAO 
inhibition assay was conducted using recombinant membrane-bound MAO A and MAO $B$ (Sigma-Aldrich, MO, USA), Kynuramine was used as substrate in 2-fold $\mathrm{K}_{M}$ concentrations $\left(K_{M}=30 \mu \mathrm{M}\right.$ for $\mathrm{MAO} A$ and $K_{M}=20 \mu \mathrm{M}$ for $\left.\mathrm{MAO} B\right)$. After initiation of enzyme reaction by addition of kynuramine, mixture was incubated $\left(20 \mathrm{~min}, 37^{\circ} \mathrm{C}\right)$ and stopped by adding $35 \mu \mathrm{L}$ sodium hydroxide $(2 \mathrm{~N})$. Enzyme activity was measured by detection of 4-hydroxyquinoline ( $\left.\lambda_{E x}=320 \pm 20 \mathrm{~nm}, \lambda_{E m}=405 \pm 20 \mathrm{~nm}\right)$ using an infinite M1000 Pro microplate reader (Tecan Trading AG, Switzerland). Data of one-point measurements were calculated as percentage of control (product formation in absence of inhibitor) and expressed as mean \pm standard deviation (\%) performing at least two independent experiments in duplicates. Clorgyline and Safinamide were used as references. 


\section{NMR Spectra}

2.1. ${ }^{1} \mathrm{H}$ and ${ }^{13} \mathrm{C}$ NMR spectra of $\beta$-lactam-oxindole hybrids

${ }^{1} \mathrm{H}$ NMR Spectra of (4aaa) $\left(400 \mathrm{MHz}, \mathrm{CDCl}_{3}\right)$ :

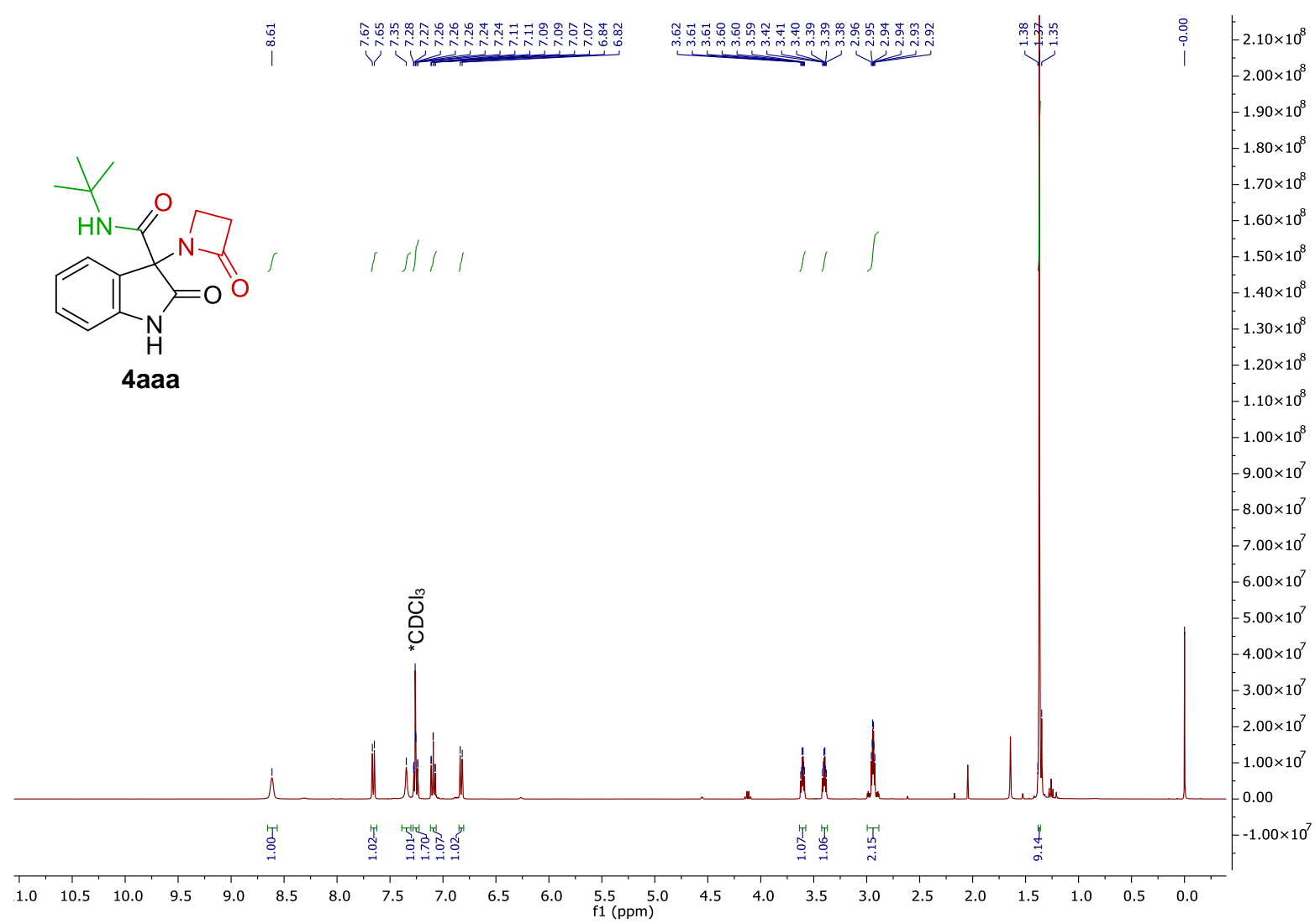

${ }^{13} \mathrm{C}$ NMR Spectra of (4aaa) (100 MHz, $\mathrm{CDCl}_{3}$ ):

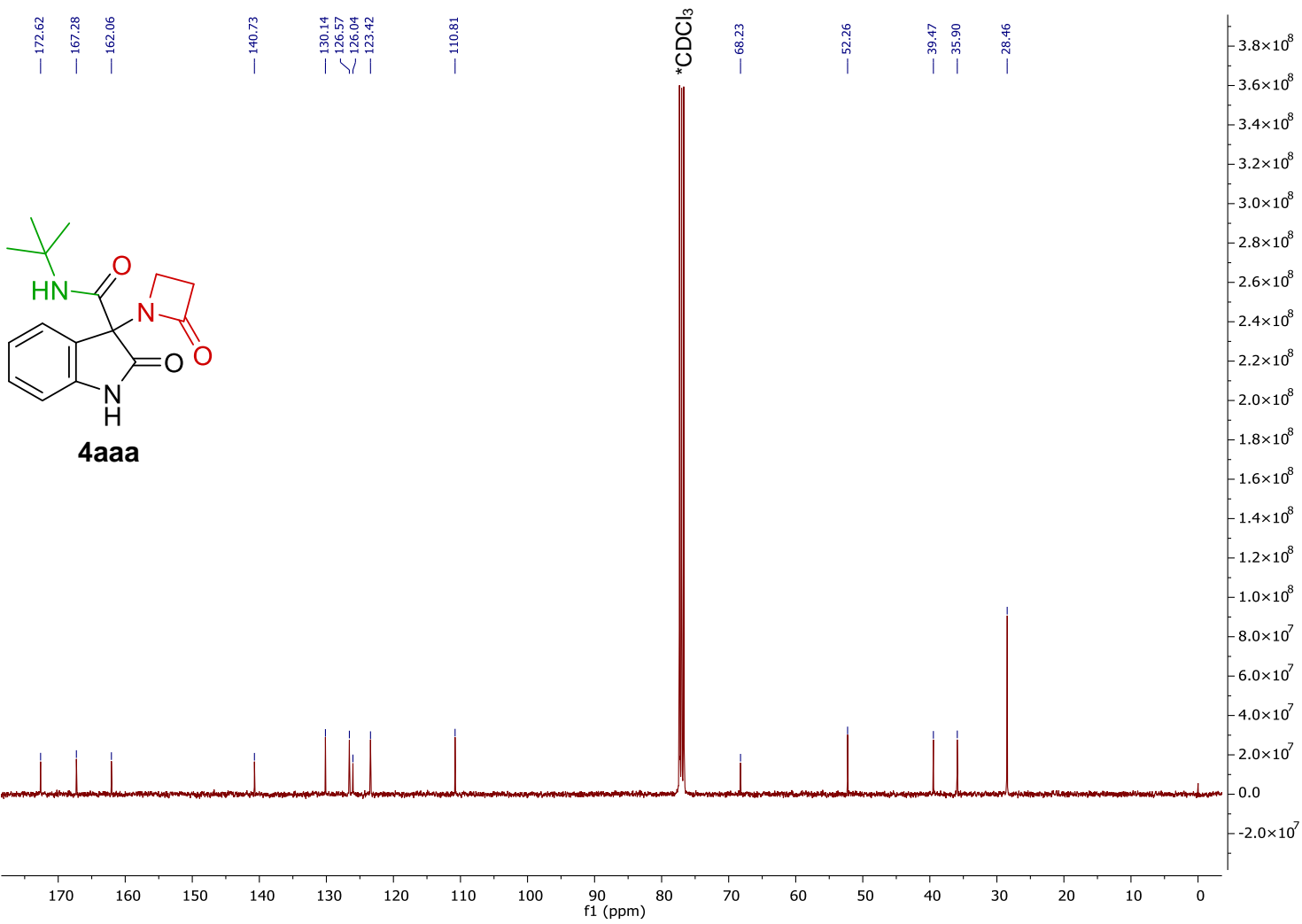


${ }^{1} \mathrm{H}$ NMR Spectra of (4baa) (400 MHz, $\left.\mathrm{CDCl}_{3}\right)$ :
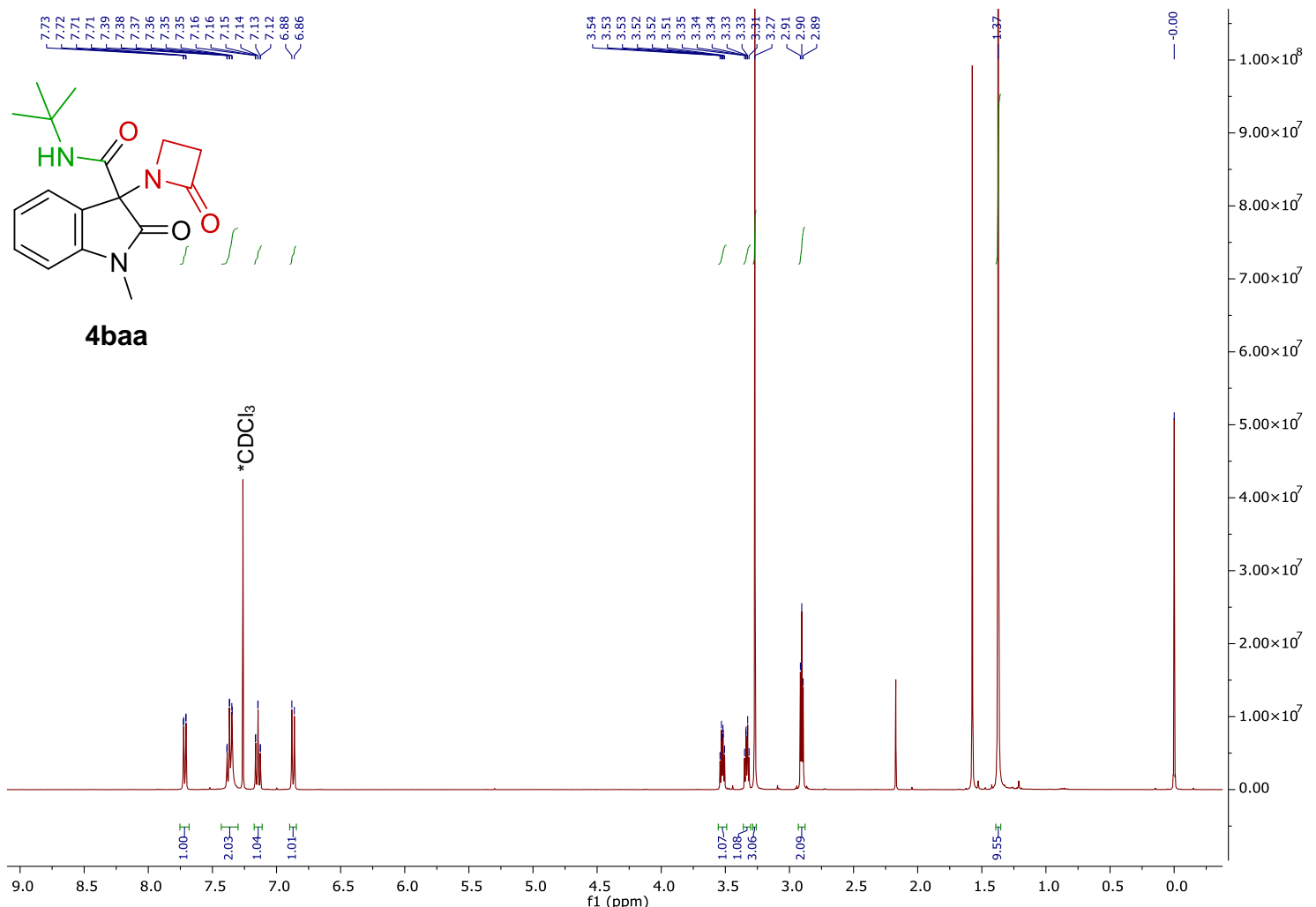

${ }^{13} \mathrm{C}$ NMR Spectra of (4baa) (100 MHz, $\mathrm{CDCl}_{3}$ ):

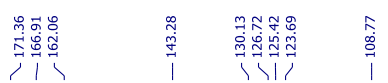

잉.

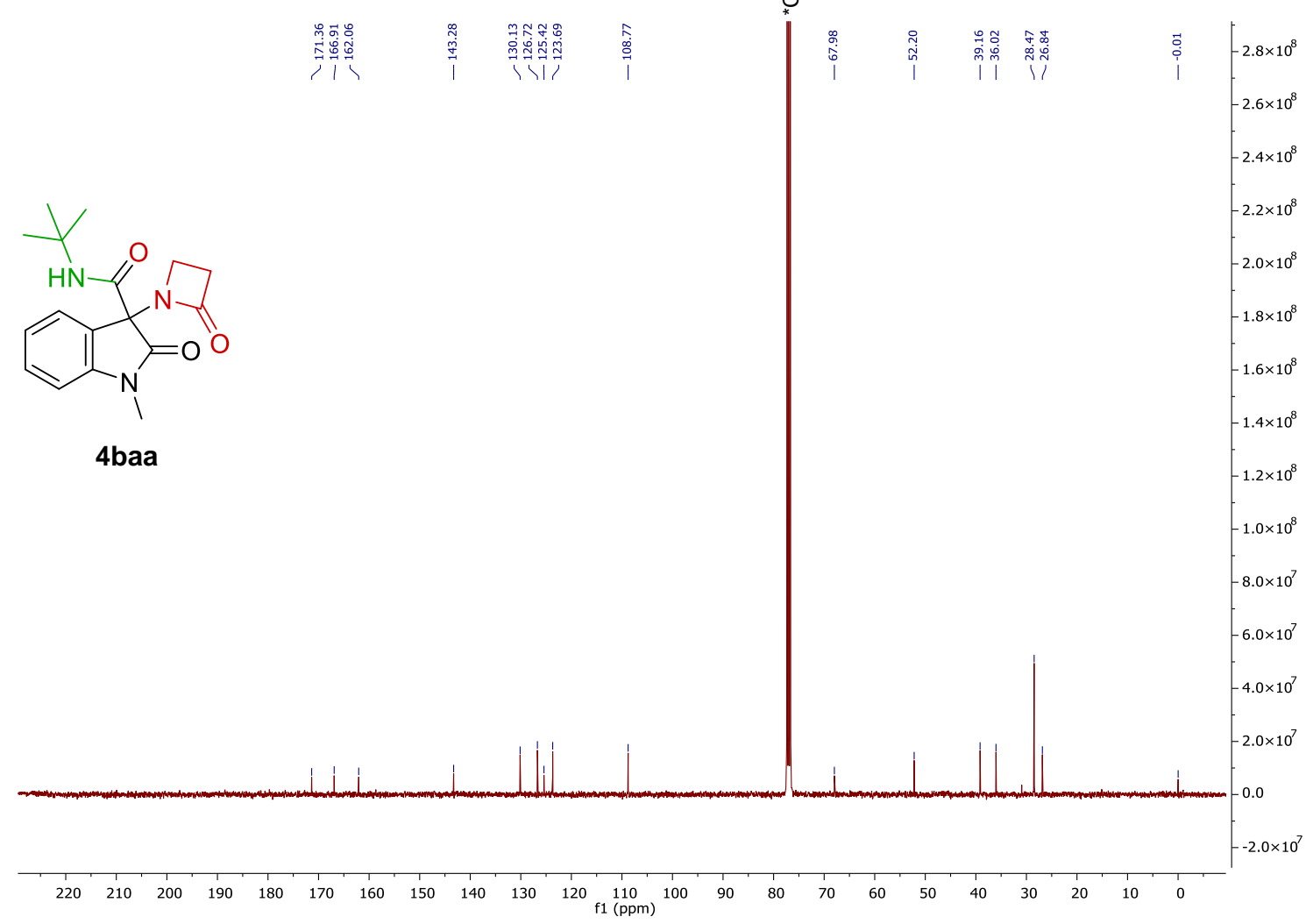

ญั

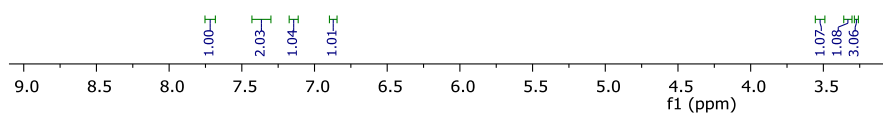


${ }^{1} \mathrm{H}$ NMR Spectra of (4caa) $\left(400 \mathrm{MHz}, \mathrm{CDCl}_{3}\right)$ :

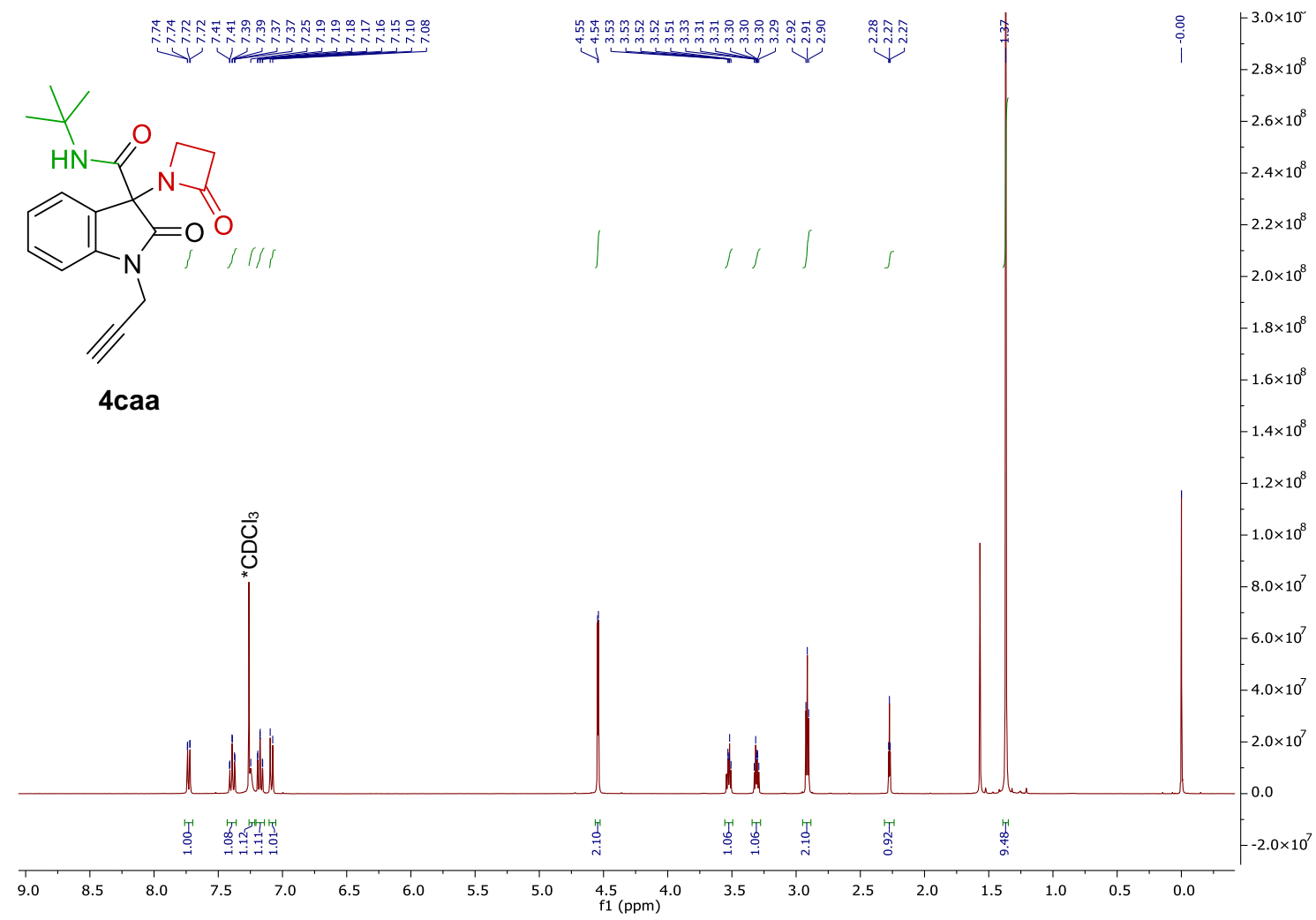

${ }^{13} \mathrm{C}$ NMR Spectra of (4caa) (100 MHz, $\left.\mathrm{CDCl}_{3}\right)$ :

品

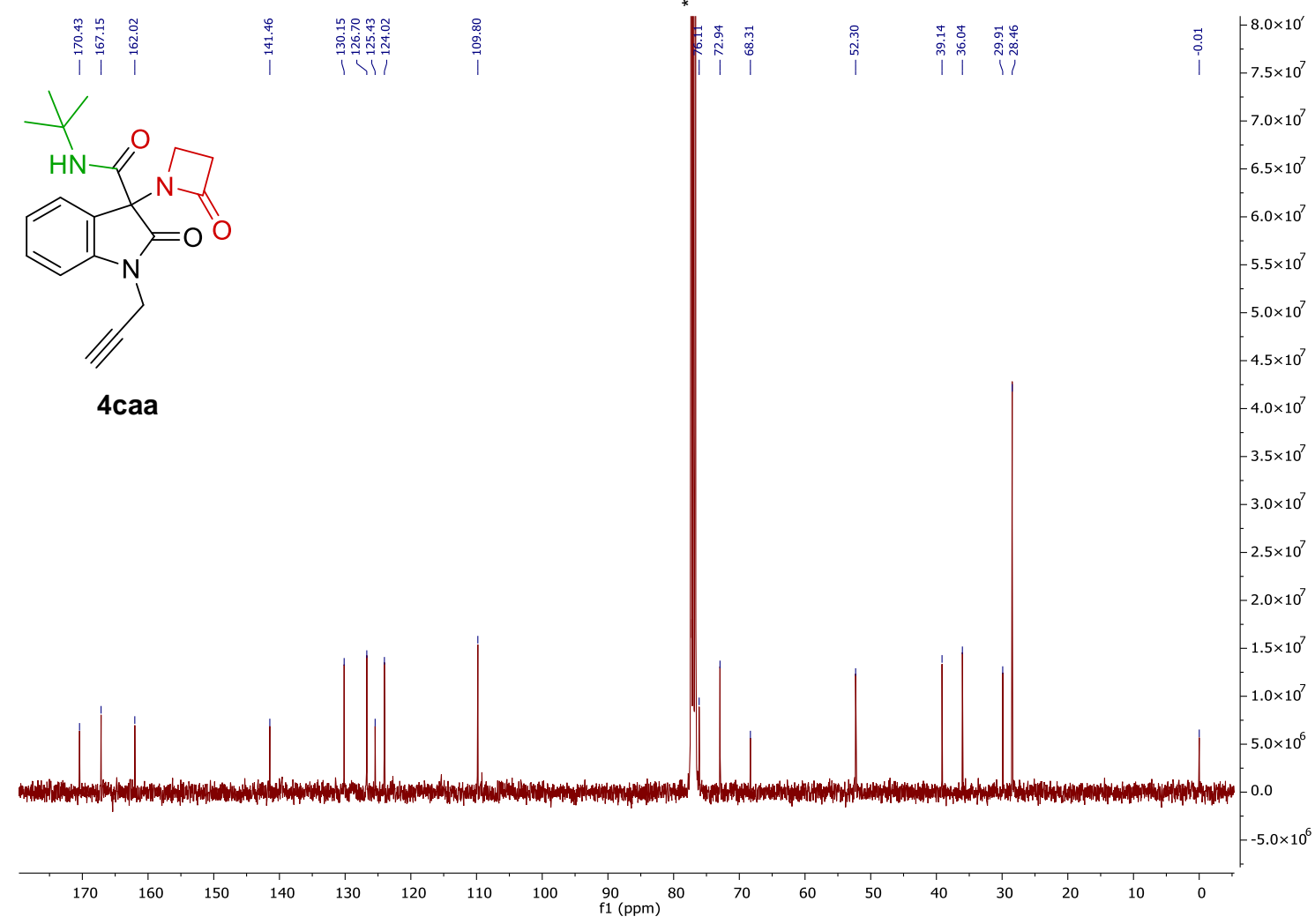


${ }^{1} \mathrm{H}$ NMR Spectra of (4daa) (400 MHz, $\mathrm{CDCl}_{3}$ ):

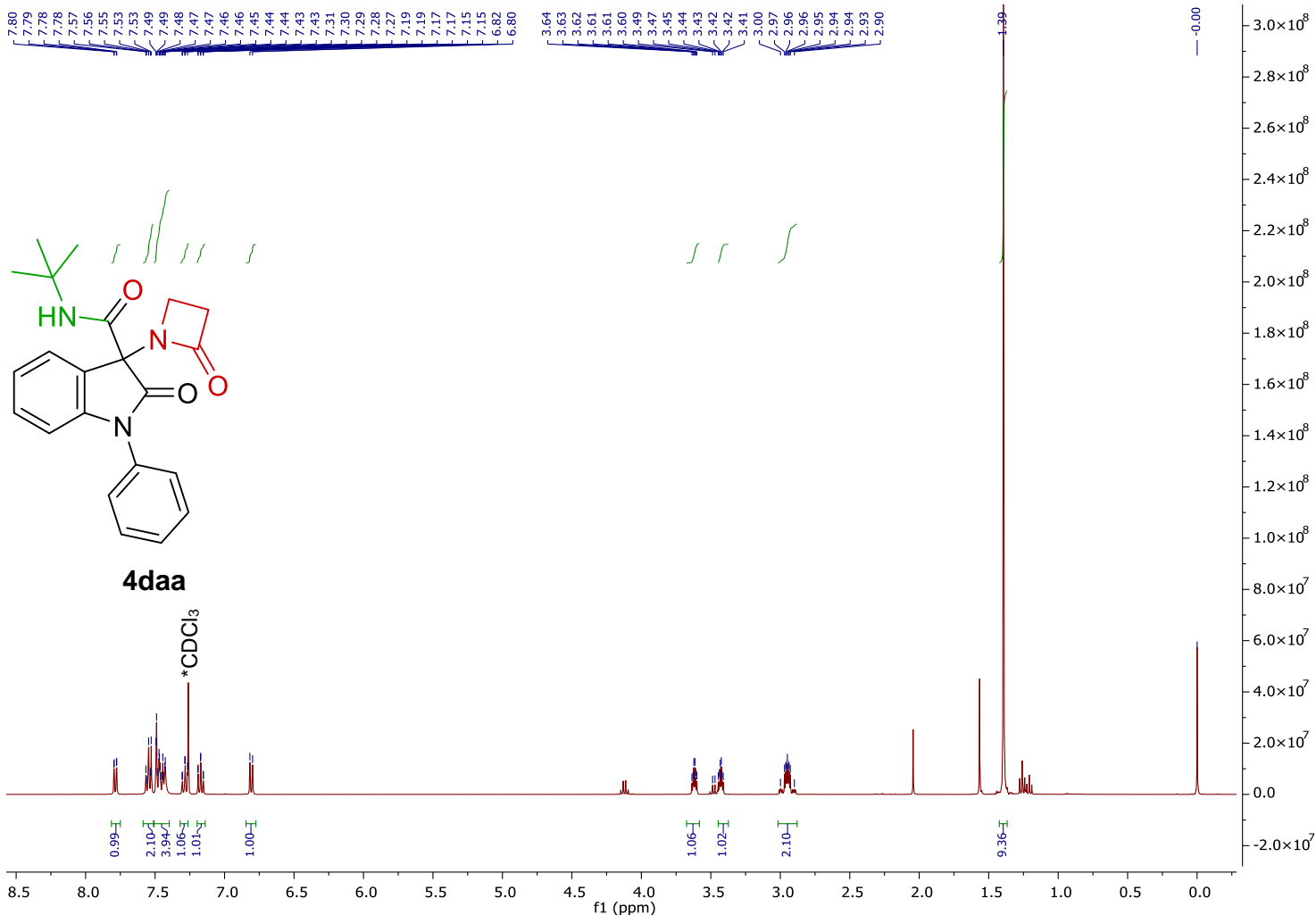

${ }^{13} \mathrm{C}$ NMR Spectra of (4daa) (100 MHz, $\mathrm{CDCl}_{3}$ ):

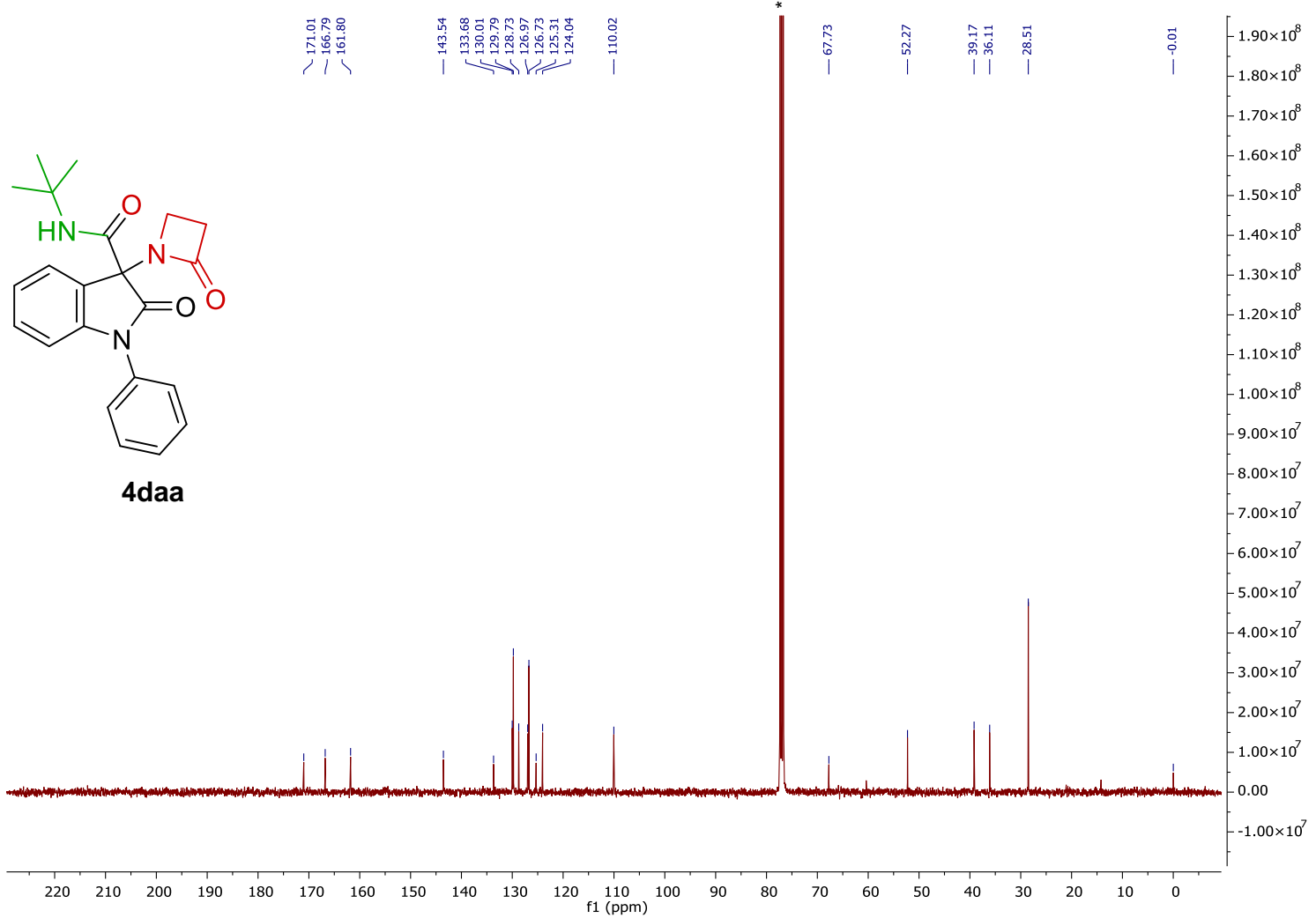


${ }^{1} \mathrm{H}$ NMR Spectra of (4eaa) $\left(400 \mathrm{MHz}, \mathrm{CDCl}_{3}\right)$ :

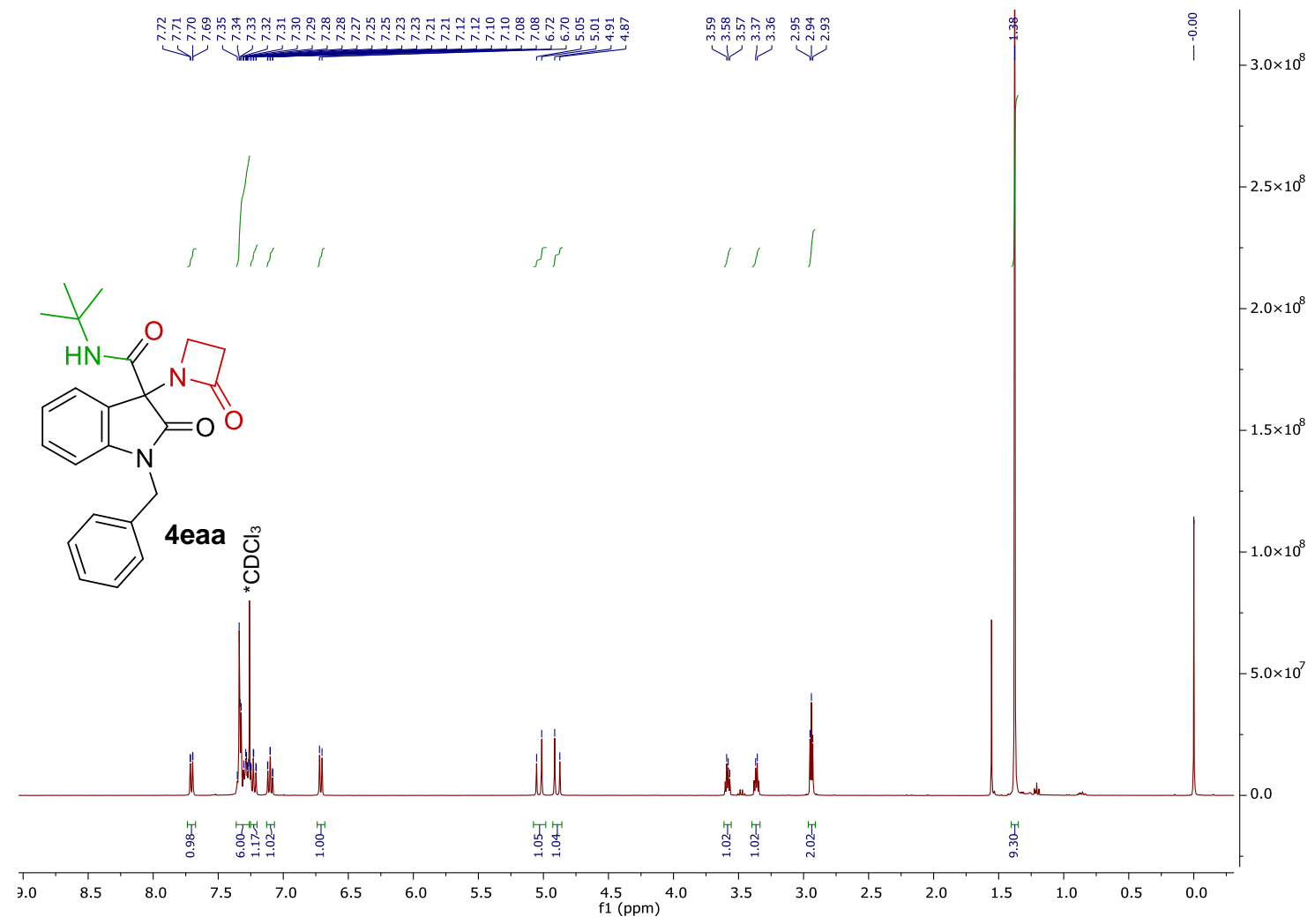

${ }^{13} \mathrm{C}$ NMR Spectra of (4eaa) (100 MHz, $\mathrm{CDCl}_{3}$ ):

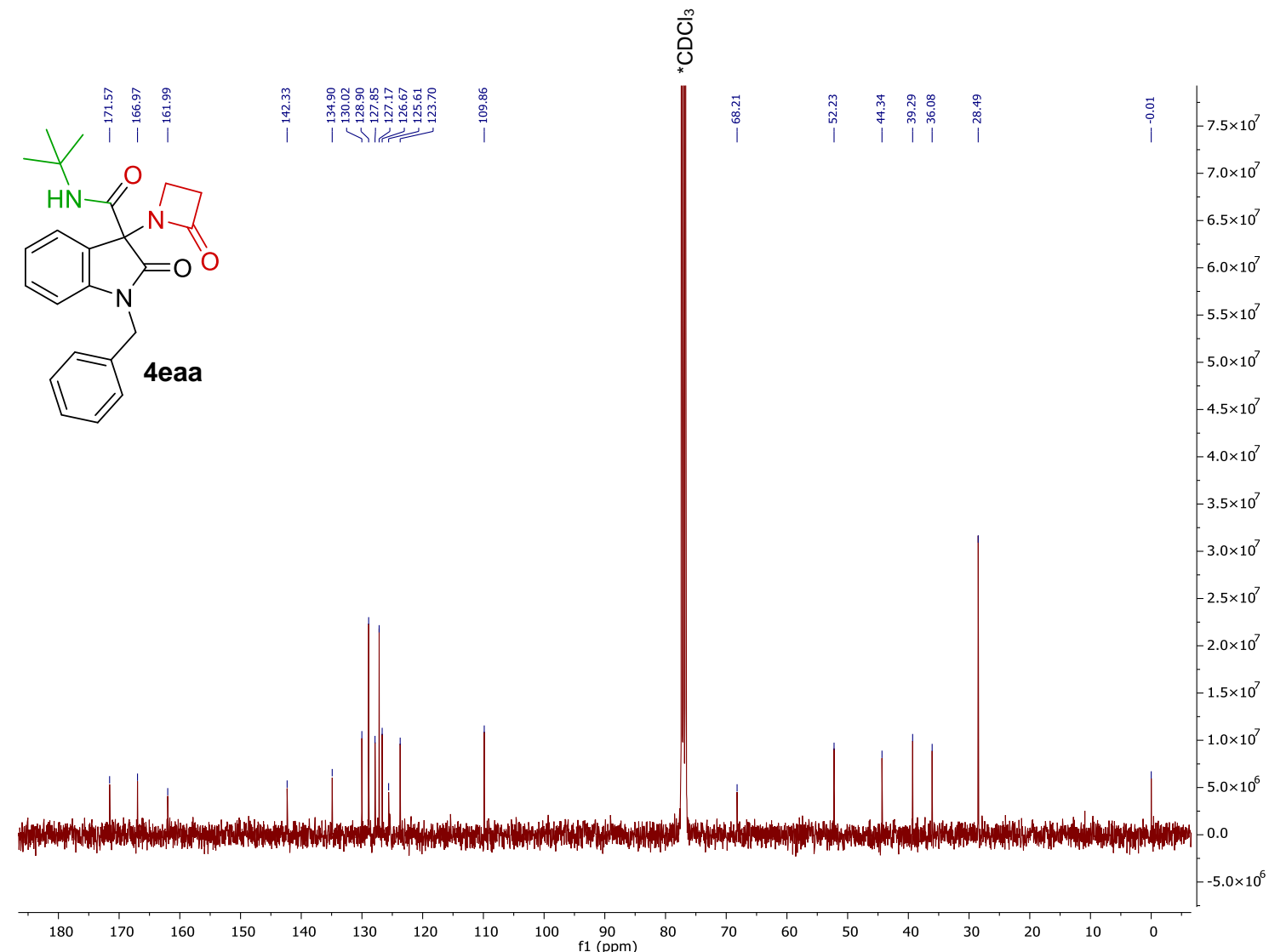


${ }^{1} \mathrm{H}$ NMR Spectra of (4faa) (400 MHz, $\left.\mathrm{CDCl}_{3}\right)$ :

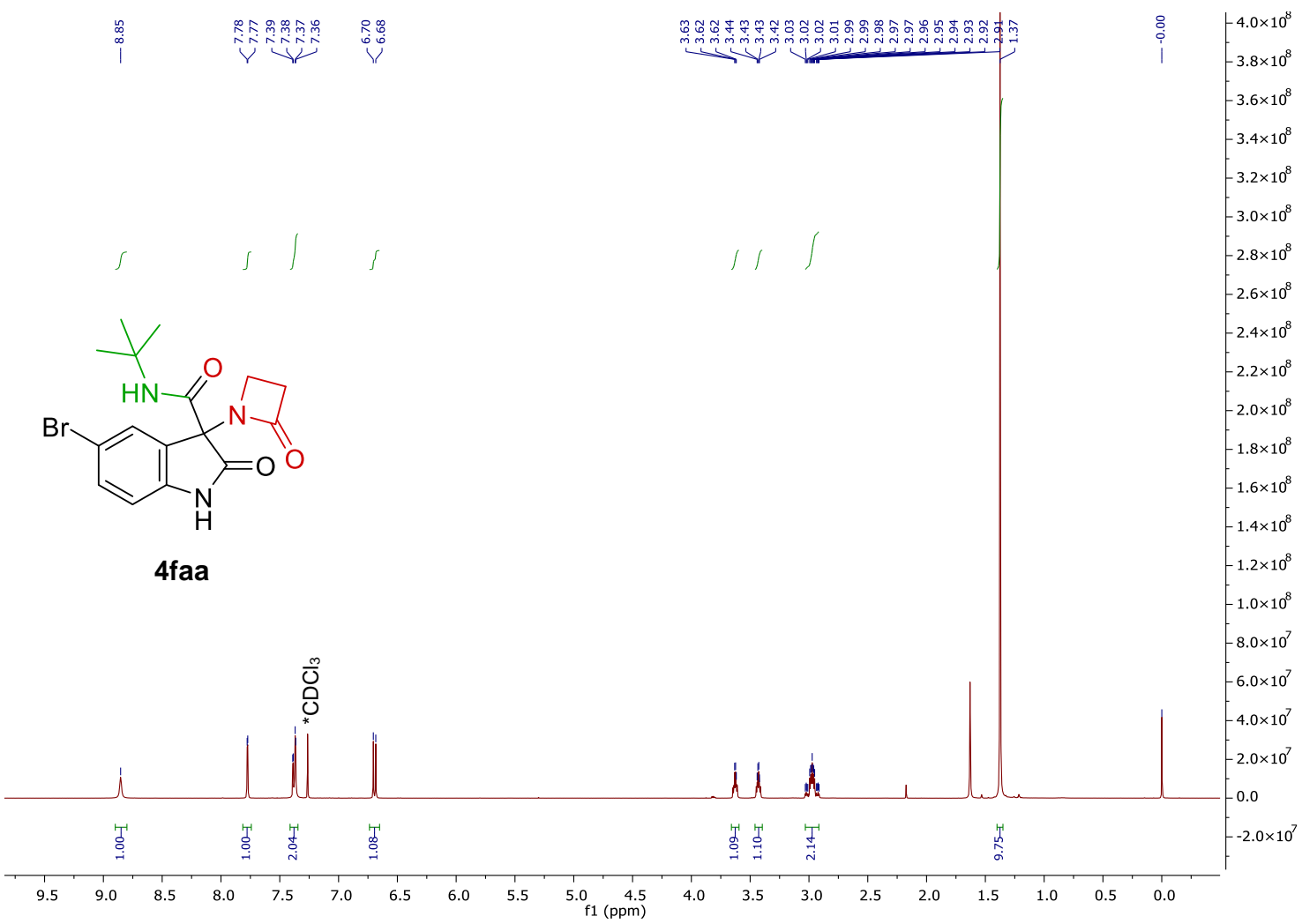

${ }^{13} \mathrm{C}$ NMR Spectra of (4faa) $\left(100 \mathrm{MHz}, \mathrm{CDCl}_{3}\right)$ :

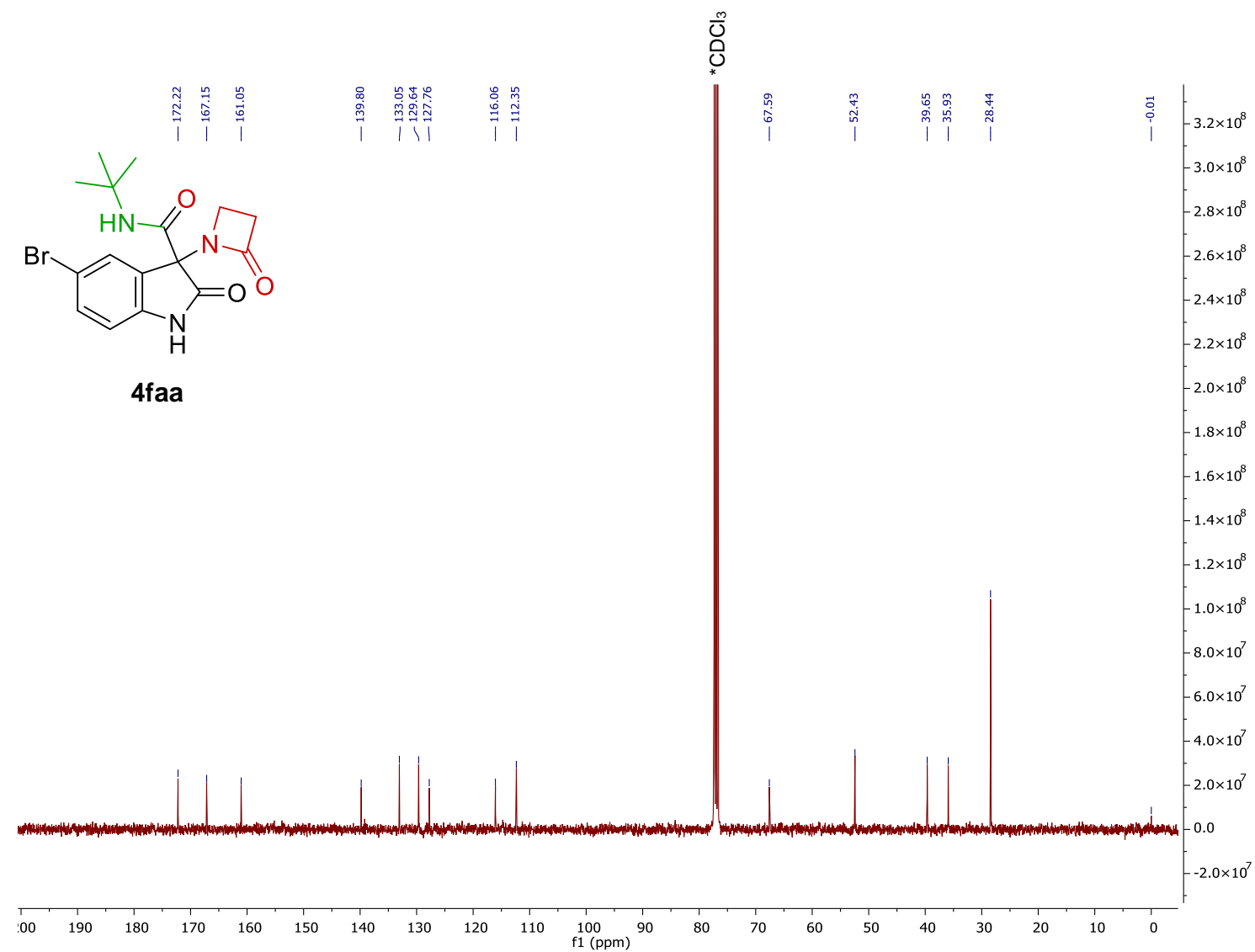


${ }^{1} \mathrm{H}$ NMR Spectra of (4gaa) (400 MHz, $\left.\mathrm{CDCl}_{3}\right)$ :

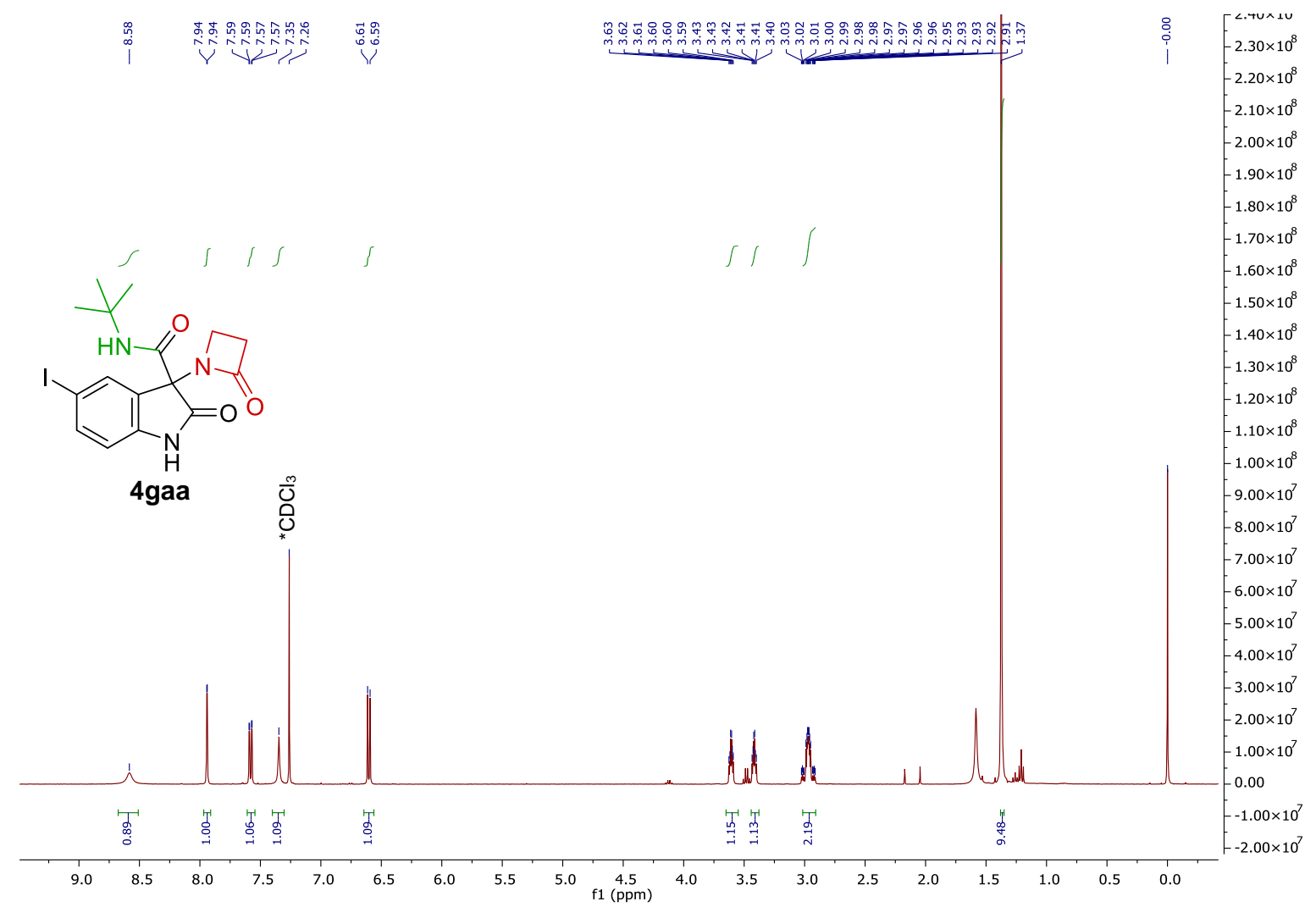

${ }^{13} \mathrm{C}$ NMR Spectra of (4gaa) (100 MHz, $\mathrm{CDCl}_{3}$ ):

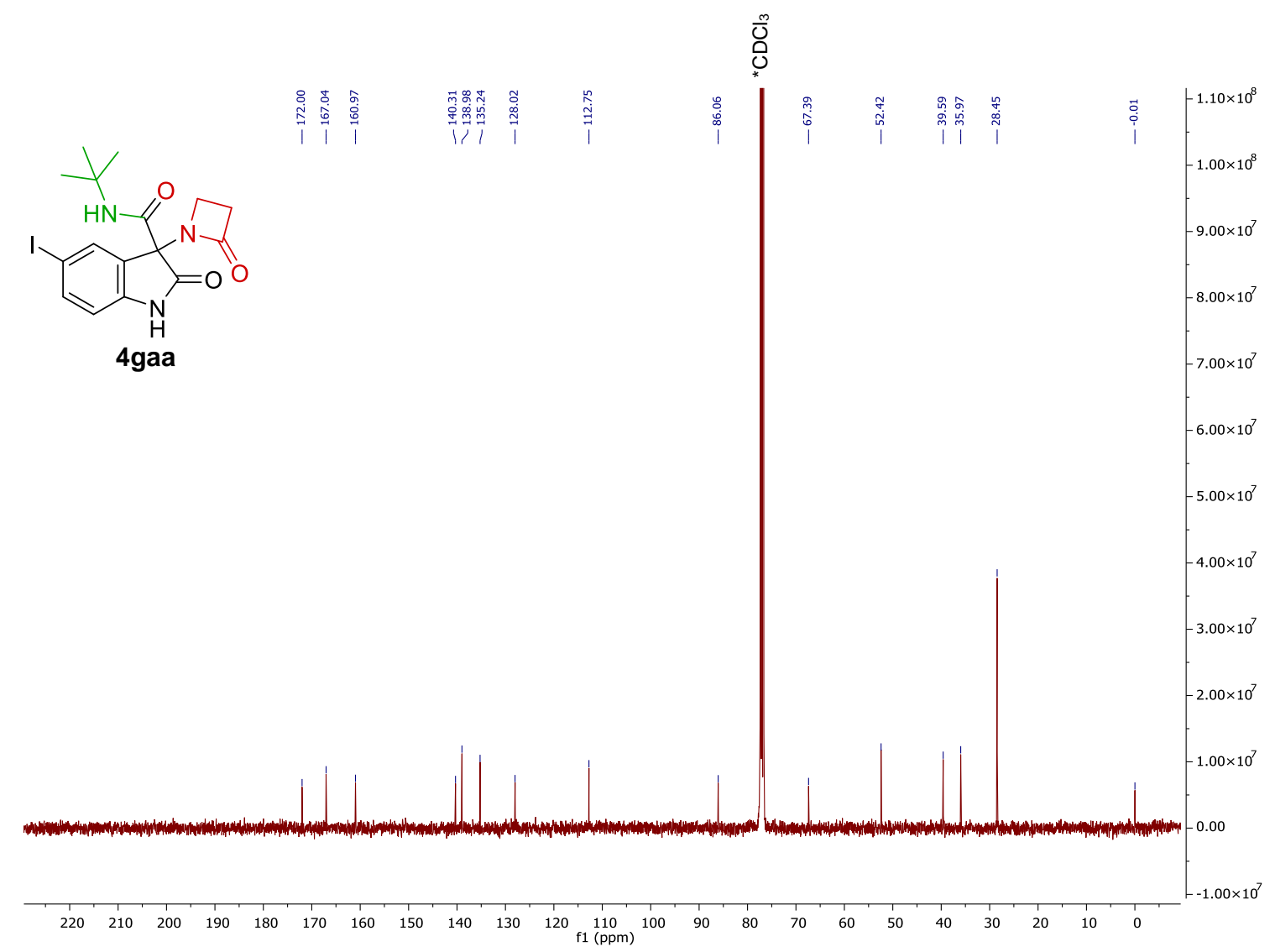


${ }^{1} \mathrm{H}$ NMR Spectra of (4haa) $\left(400 \mathrm{MHz},\left(\mathrm{CD}_{3}\right)_{2} \mathrm{CO}\right)$ :
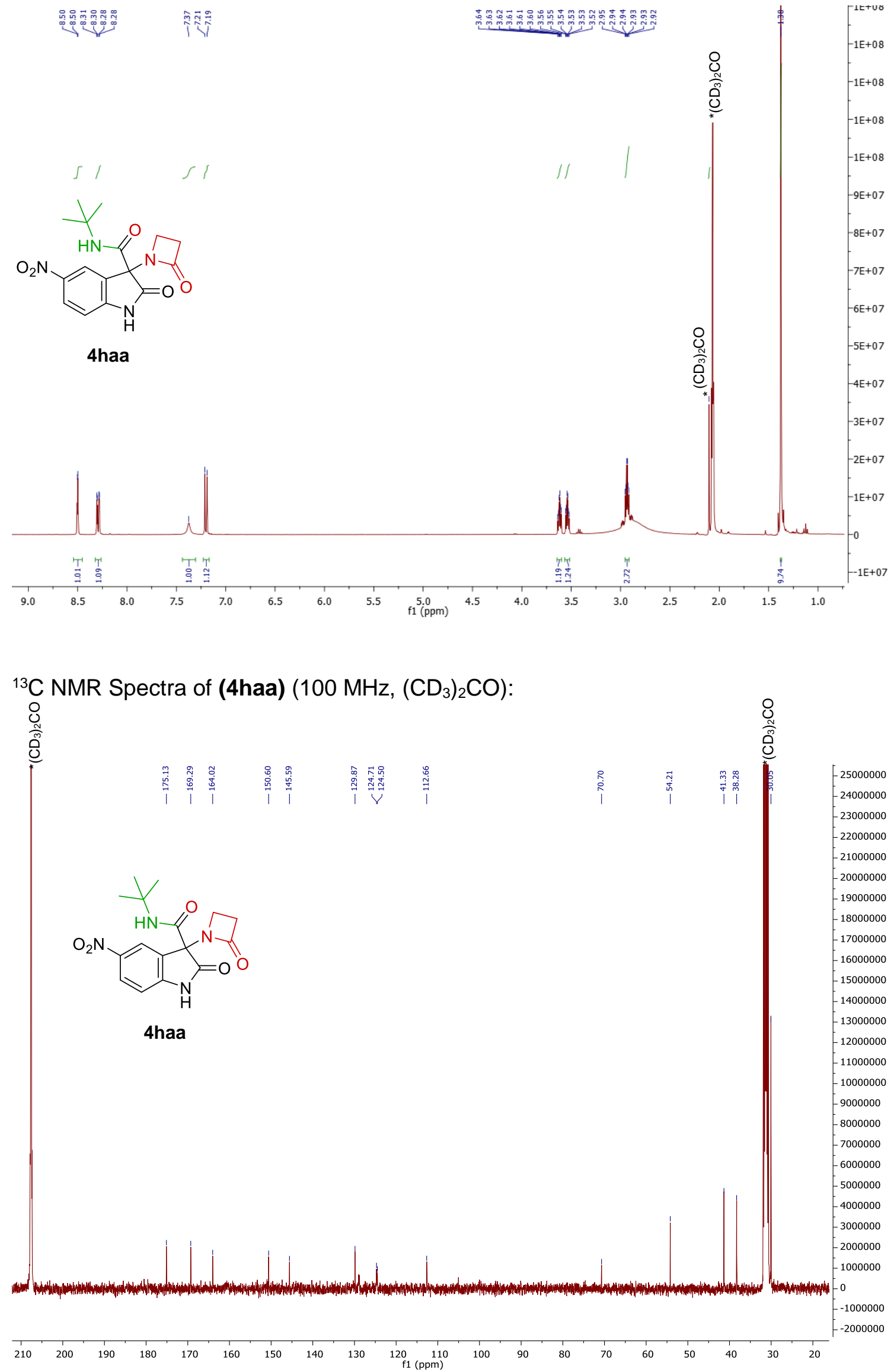
${ }^{1} \mathrm{H}$ NMR Spectra of (4iaa) (400 MHz, $\mathrm{CDCl}_{3}$ ):

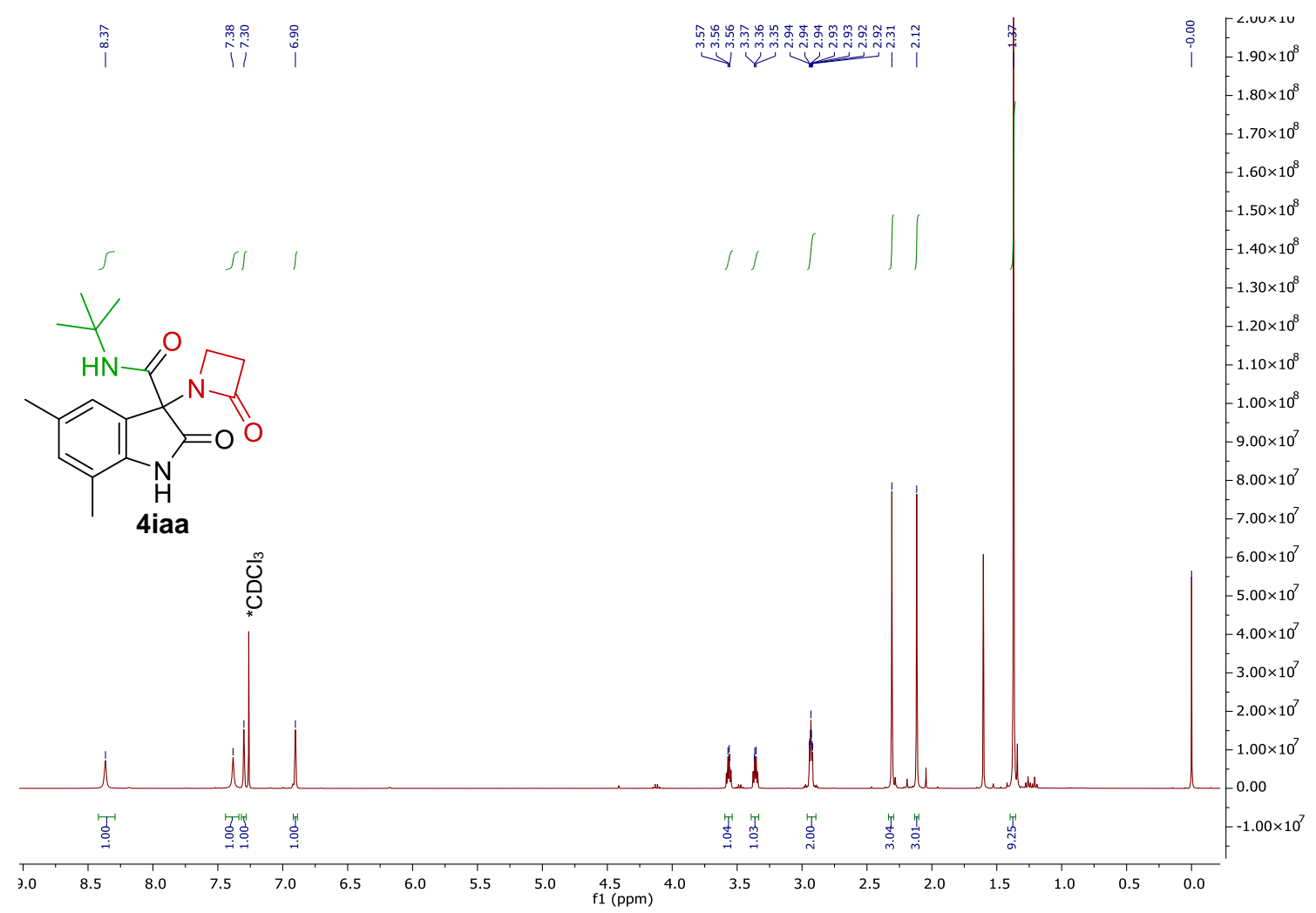

${ }^{13} \mathrm{C}$ NMR Spectra of (4iaa) (100 MHz, $\left.\mathrm{CDCl}_{3}\right)$ :

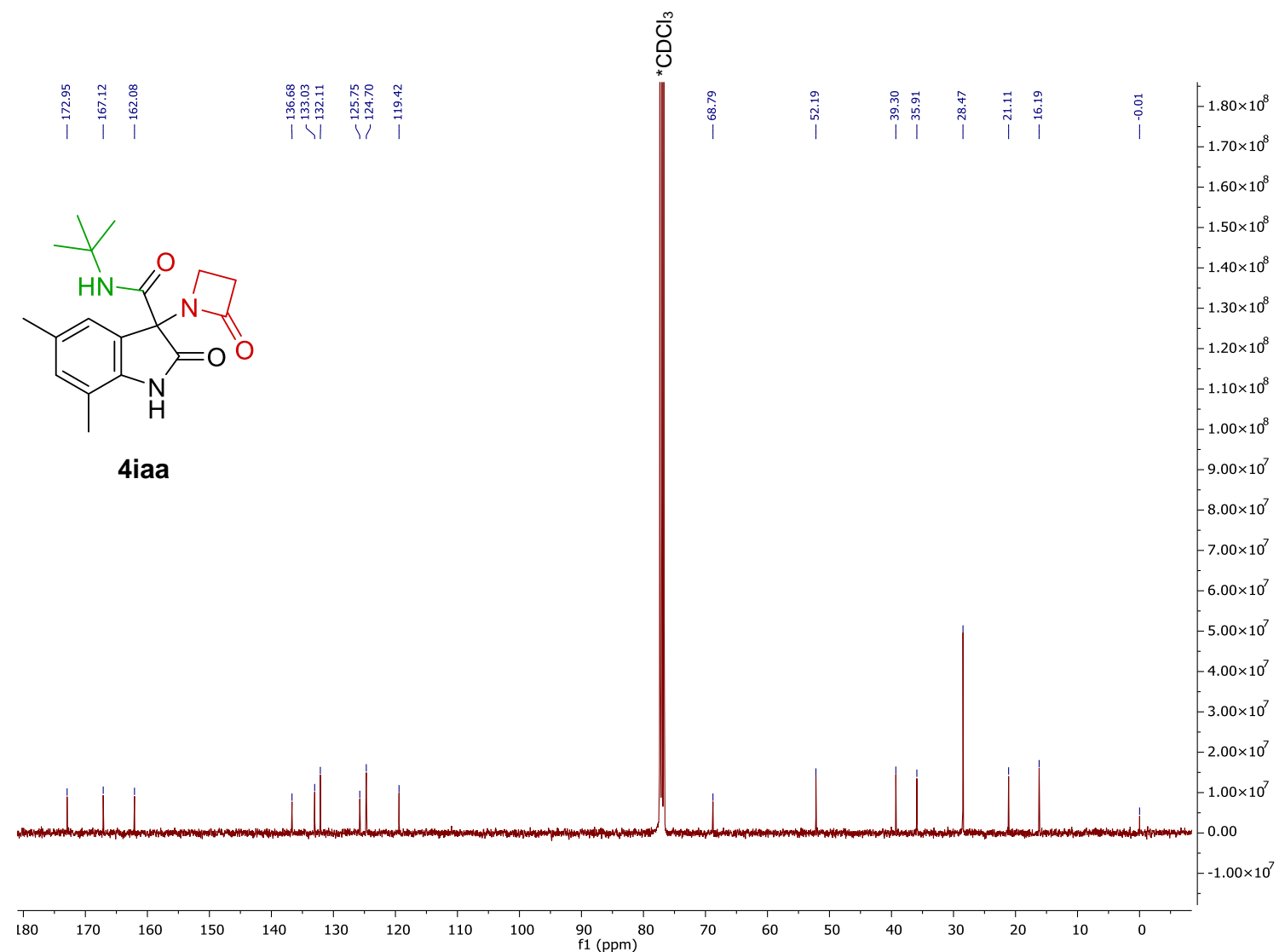


${ }^{1} \mathrm{H}$ NMR Spectra of (4jaa) (400 MHz, $\left.\mathrm{CDCl}_{3}\right)$ :

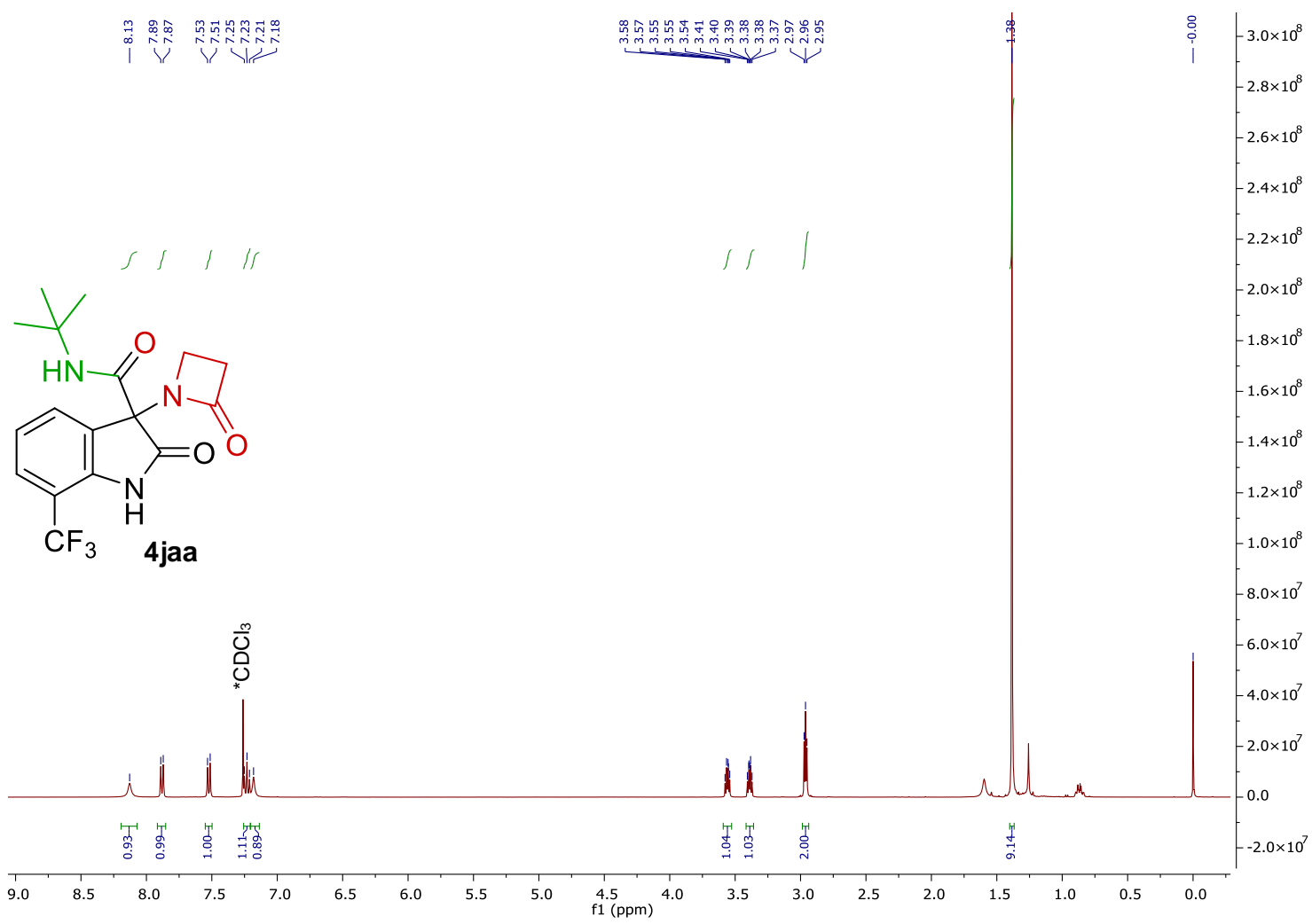

${ }^{13} \mathrm{C}$ NMR Spectra of (4jaa) $\left(100 \mathrm{MHz}, \mathrm{CDCl}_{3}\right)$ :

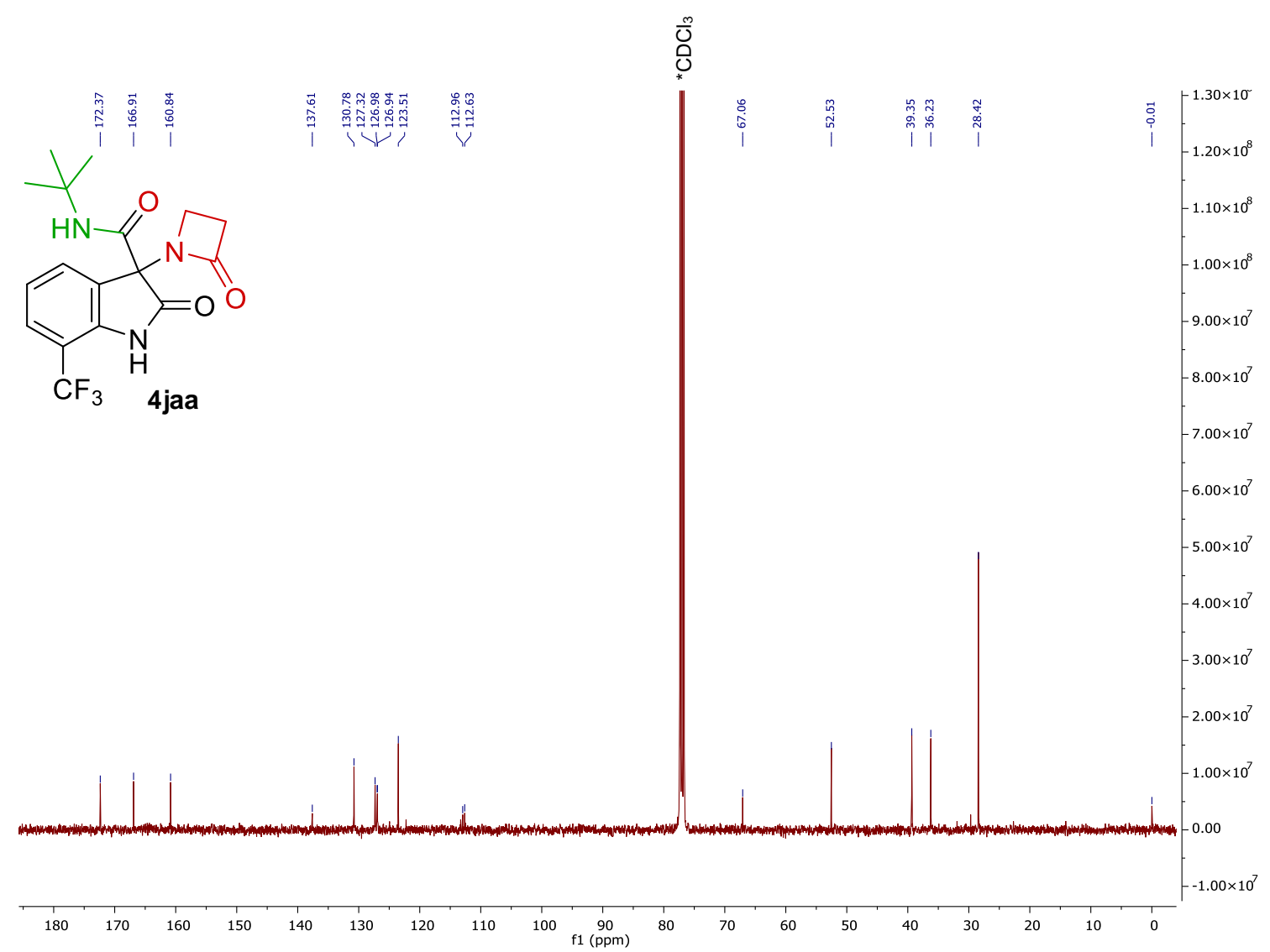


${ }^{1} \mathrm{H}$ NMR Spectra of (4aba) (400 MHz, $\left.\mathrm{CDCl}_{3}\right)$ :

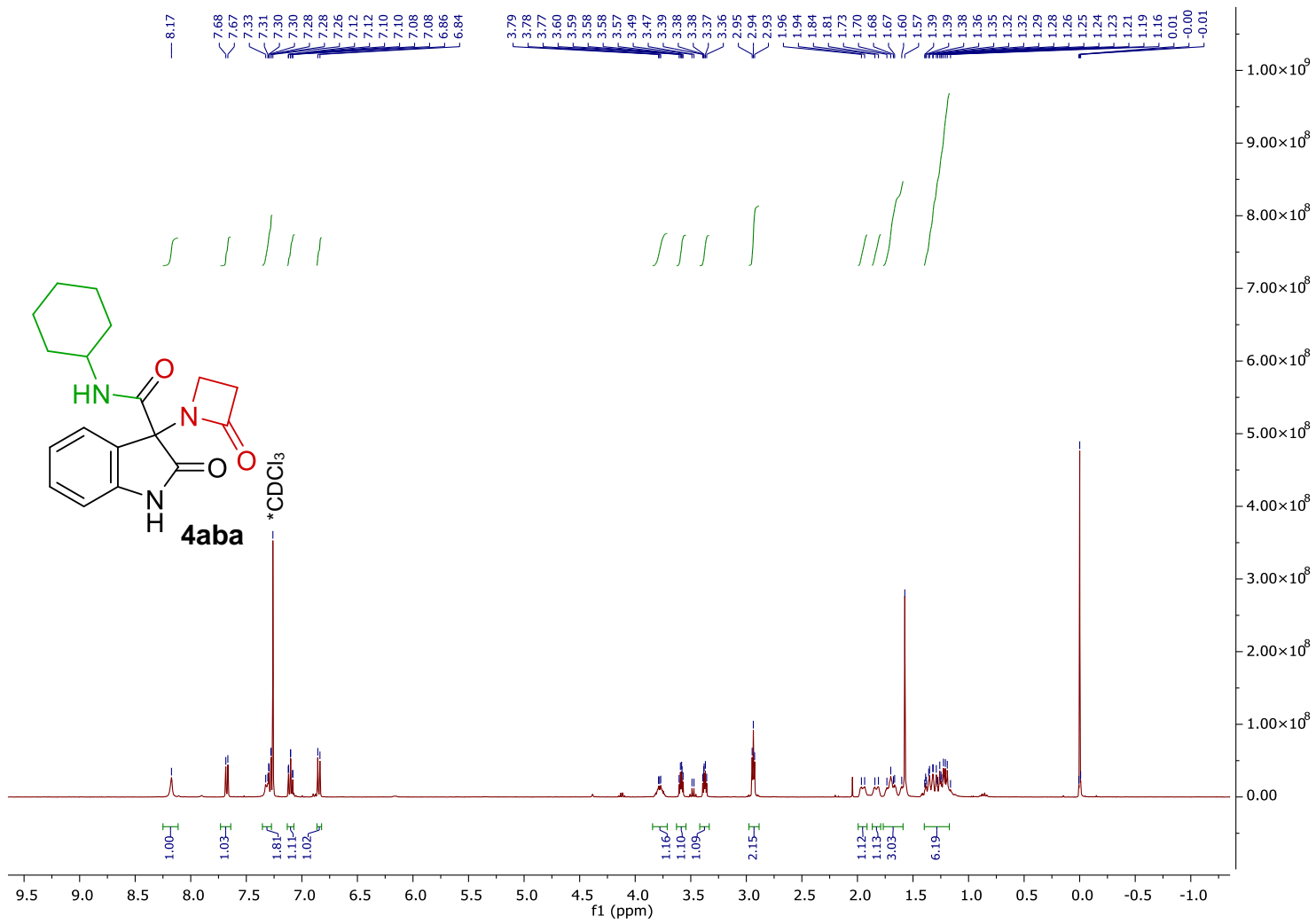

${ }^{13} \mathrm{C}$ NMR Spectra of (4aba) (100 MHz, $\mathrm{CDCl}_{3}$ ):

离

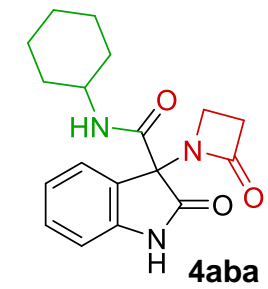

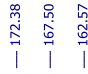

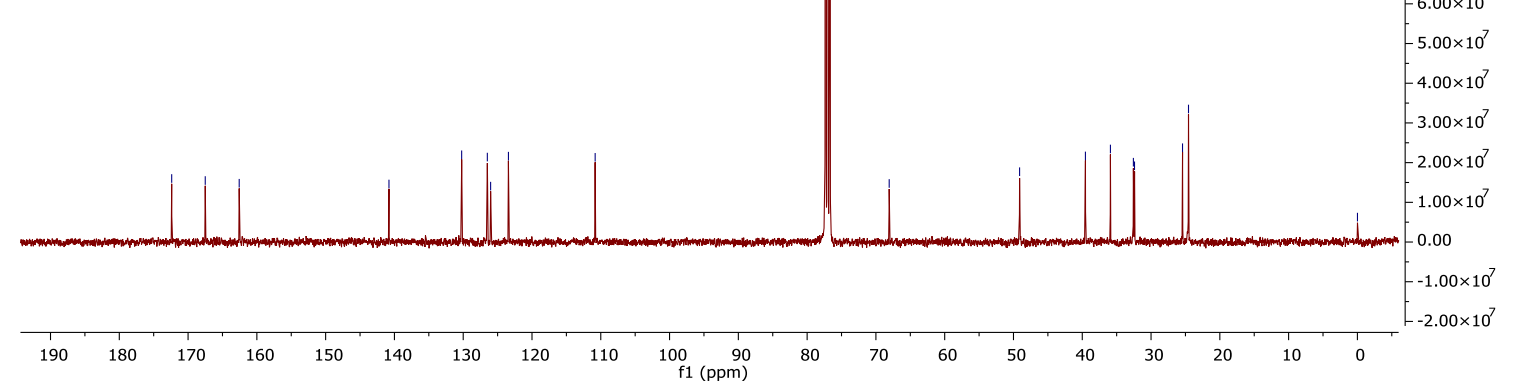


${ }^{1} \mathrm{H}$ NMR Spectra of (4aca) $\left(400 \mathrm{MHz}, \mathrm{CDCl}_{3}\right)$ :

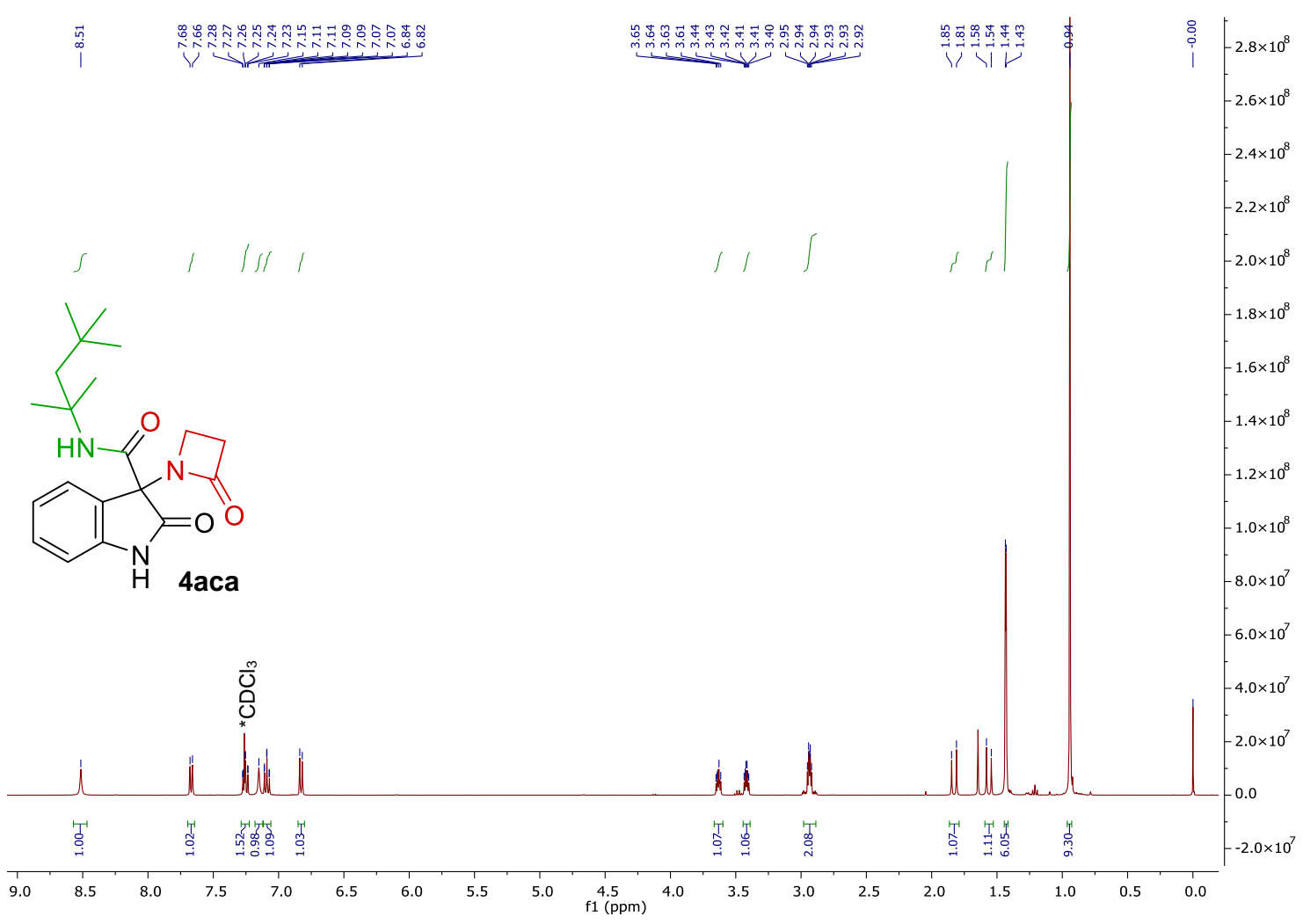

${ }^{13} \mathrm{C}$ NMR Spectra of (4aca) (100 MHz, $\mathrm{CDCl}_{3}$ ):

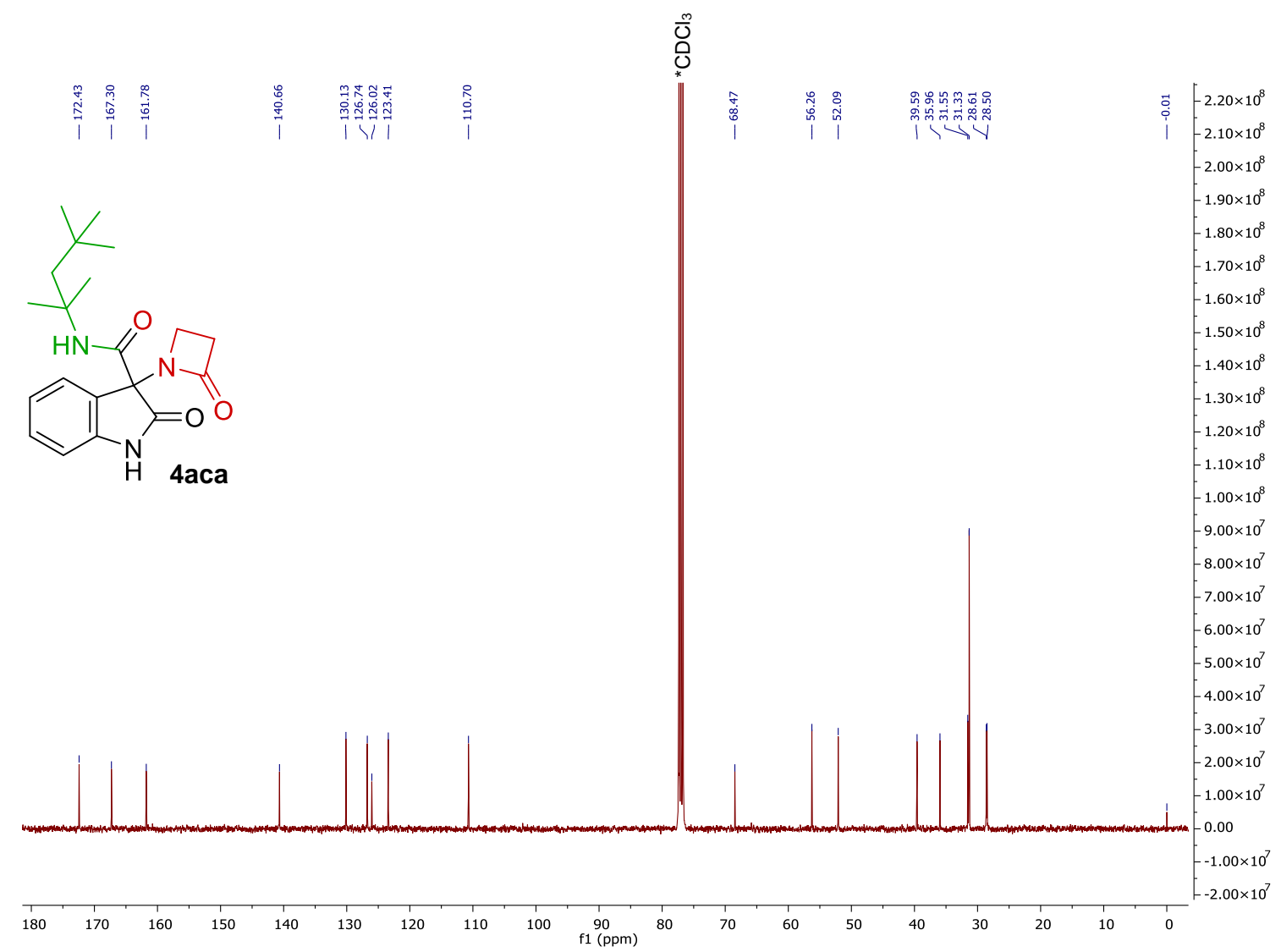


${ }^{1} \mathrm{H}$ NMR Spectra of (4ada) (400 MHz, $\left.\mathrm{CDCl}_{3}\right)$ :

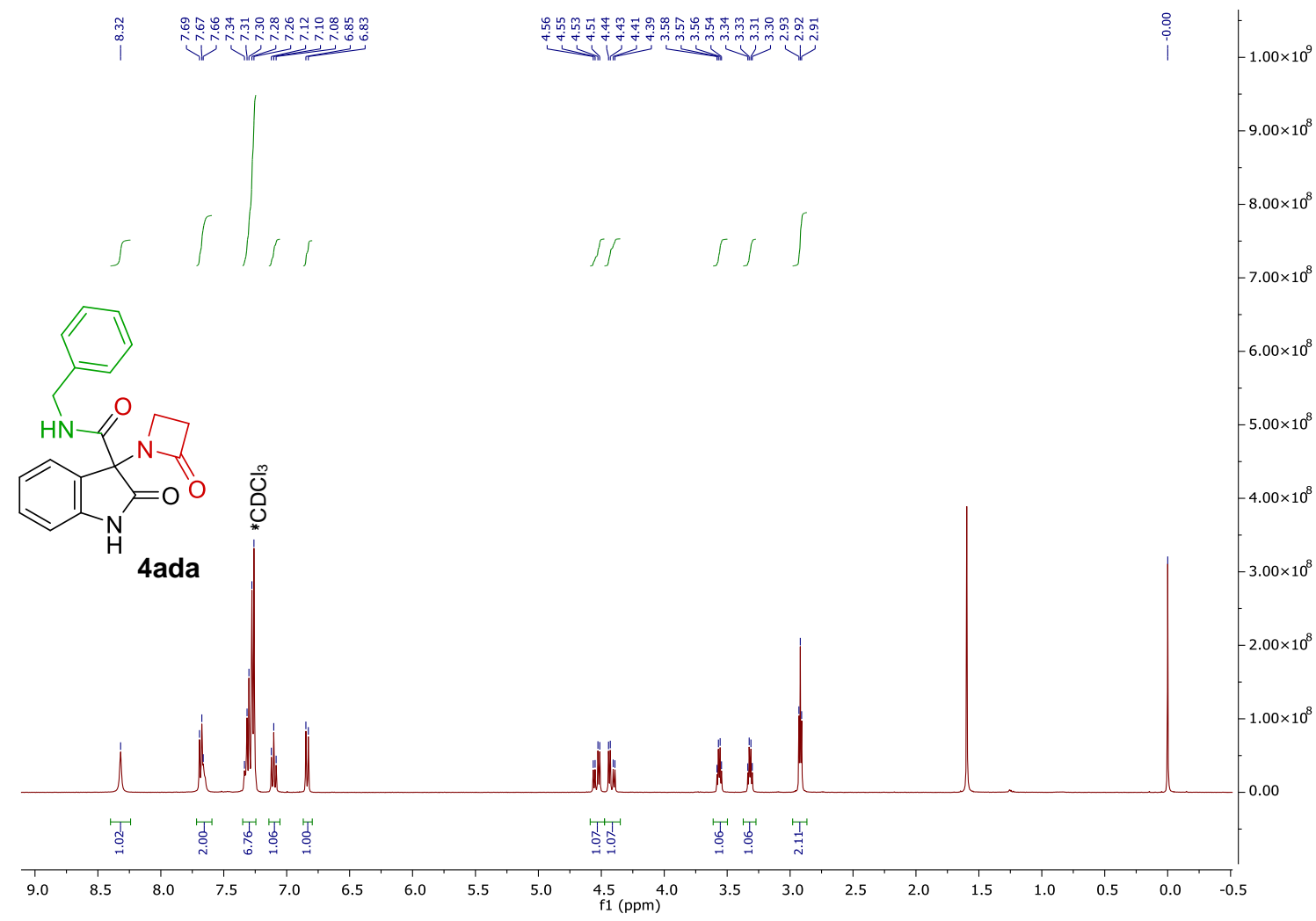

${ }^{13} \mathrm{C}$ NMR Spectra of (4ada) (100 MHz, $\mathrm{CDCl}_{3}$ ):

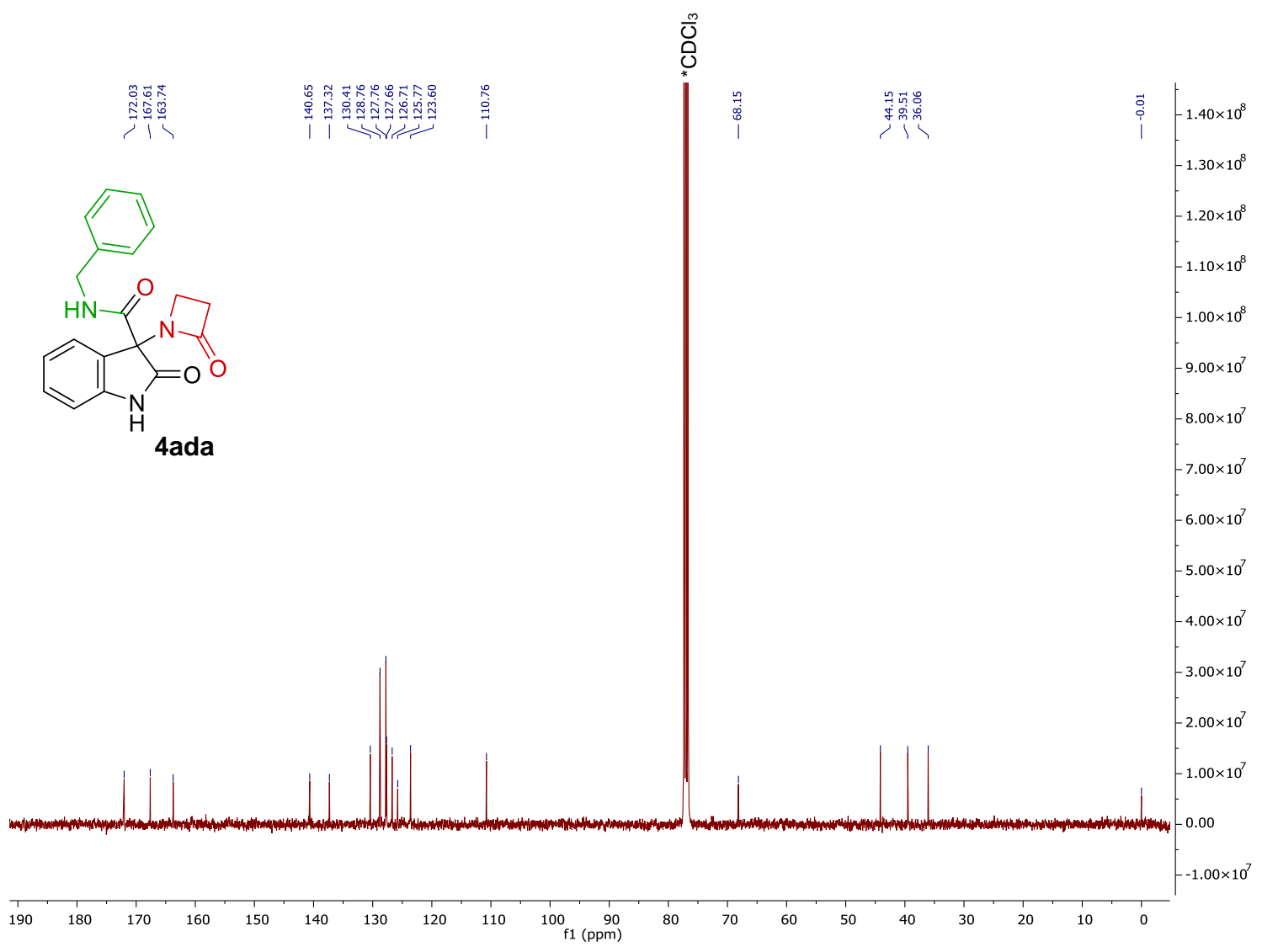


${ }^{1} \mathrm{H}$ NMR Spectra of (4eca) $\left(400 \mathrm{MHz}, \mathrm{CDCl}_{3}\right)$ :

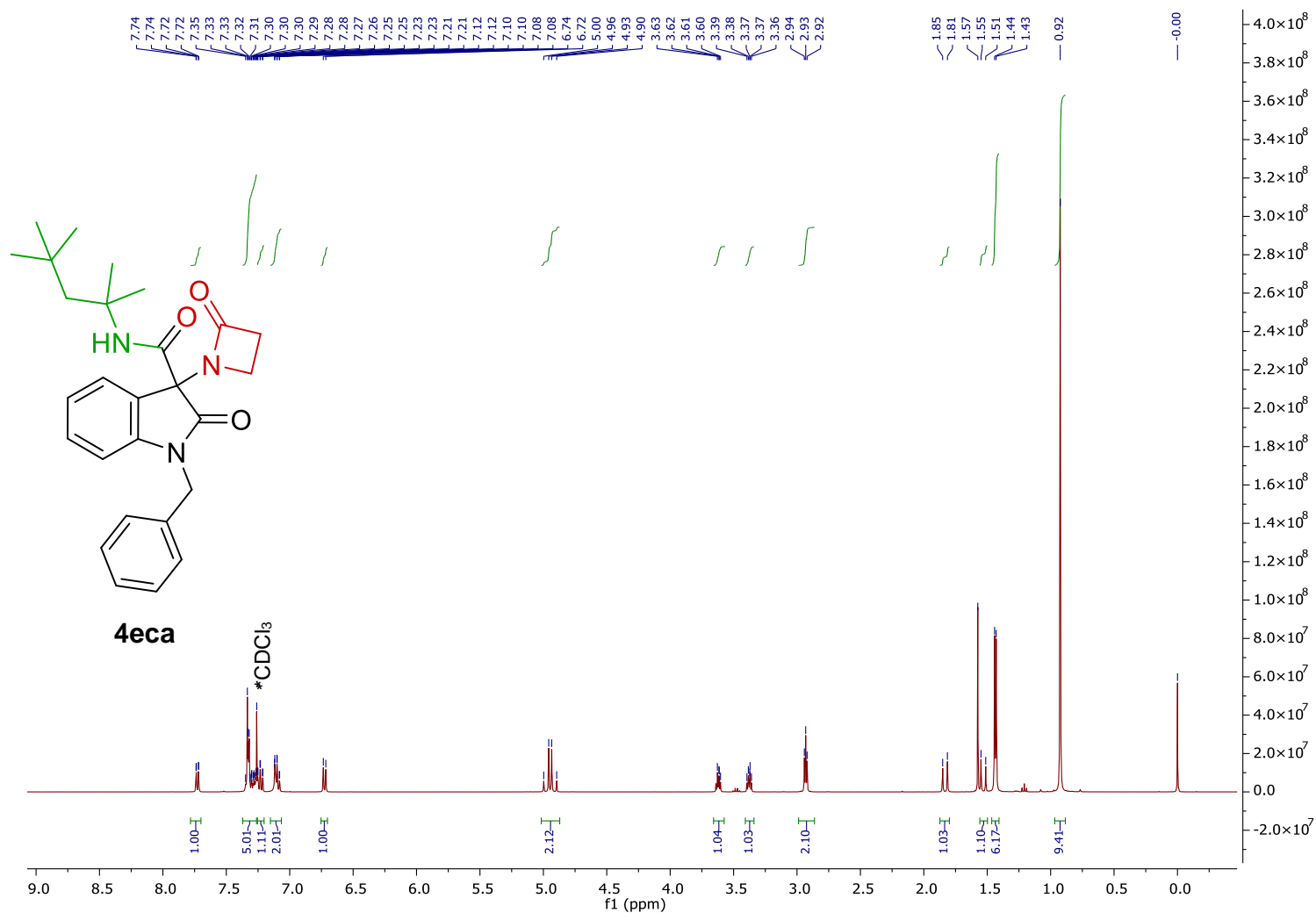


${ }^{1} \mathrm{H}$ NMR Spectra of (4cda) $\left(400 \mathrm{MHz}, \mathrm{CDCl}_{3}\right)$ :

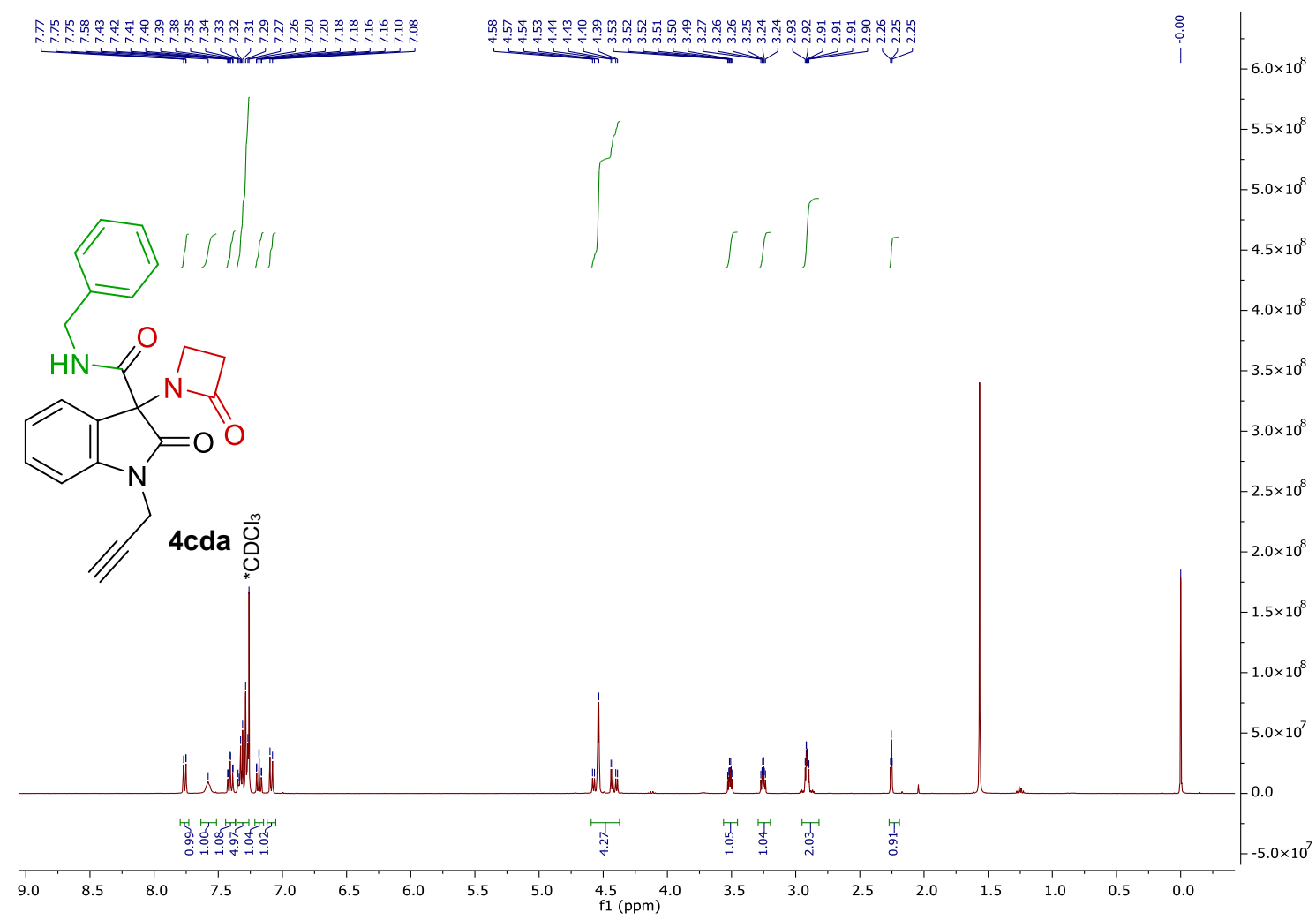

${ }^{13} \mathrm{C}$ NMR Spectra of (4cda) (100 MHz, $\left.\mathrm{CDCl}_{3}\right)$ :

잉

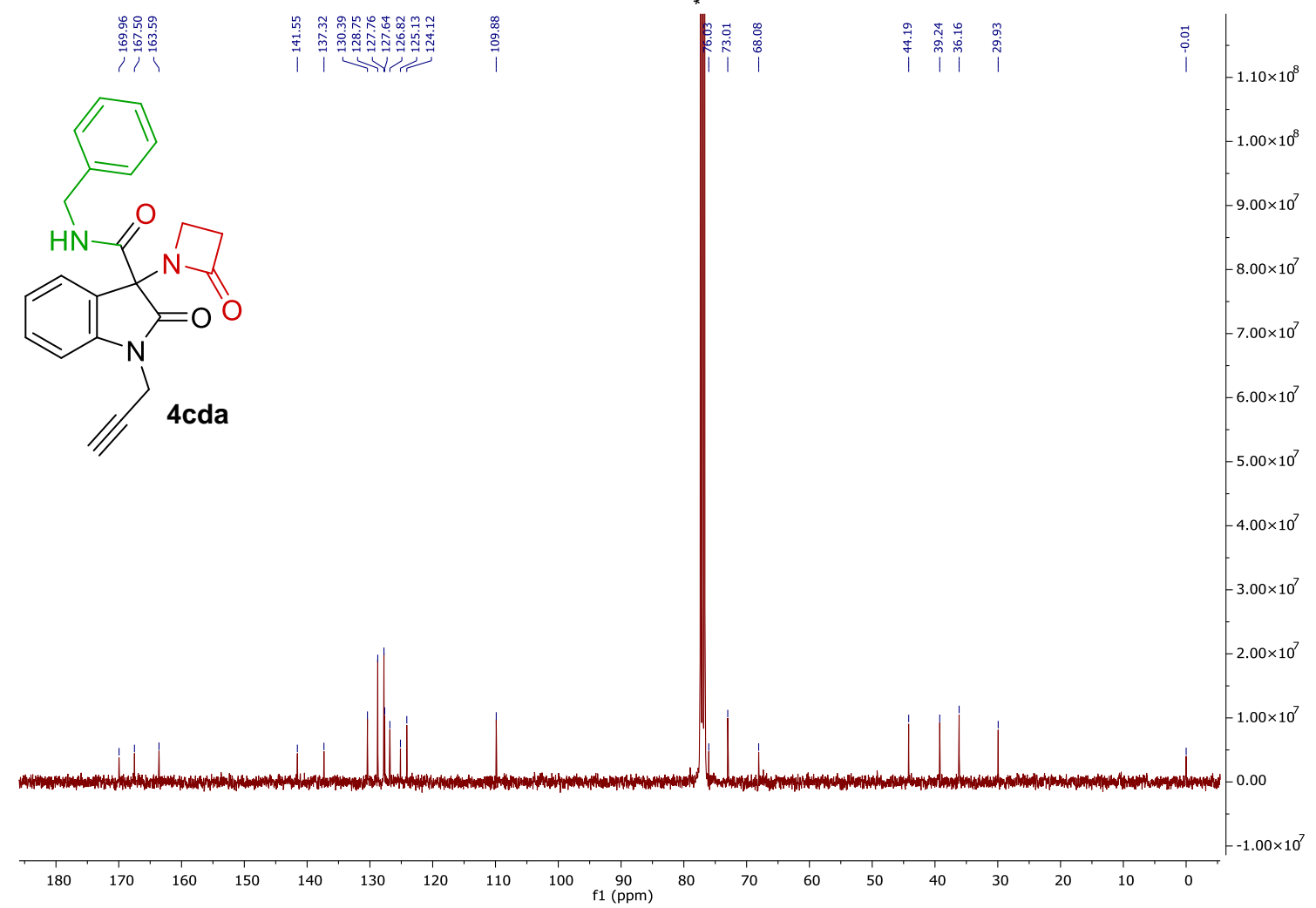


${ }^{1} \mathrm{H}$ NMR Spectra of (4bba) $\left(400 \mathrm{MHz}, \mathrm{CDCl}_{3}\right)$ :

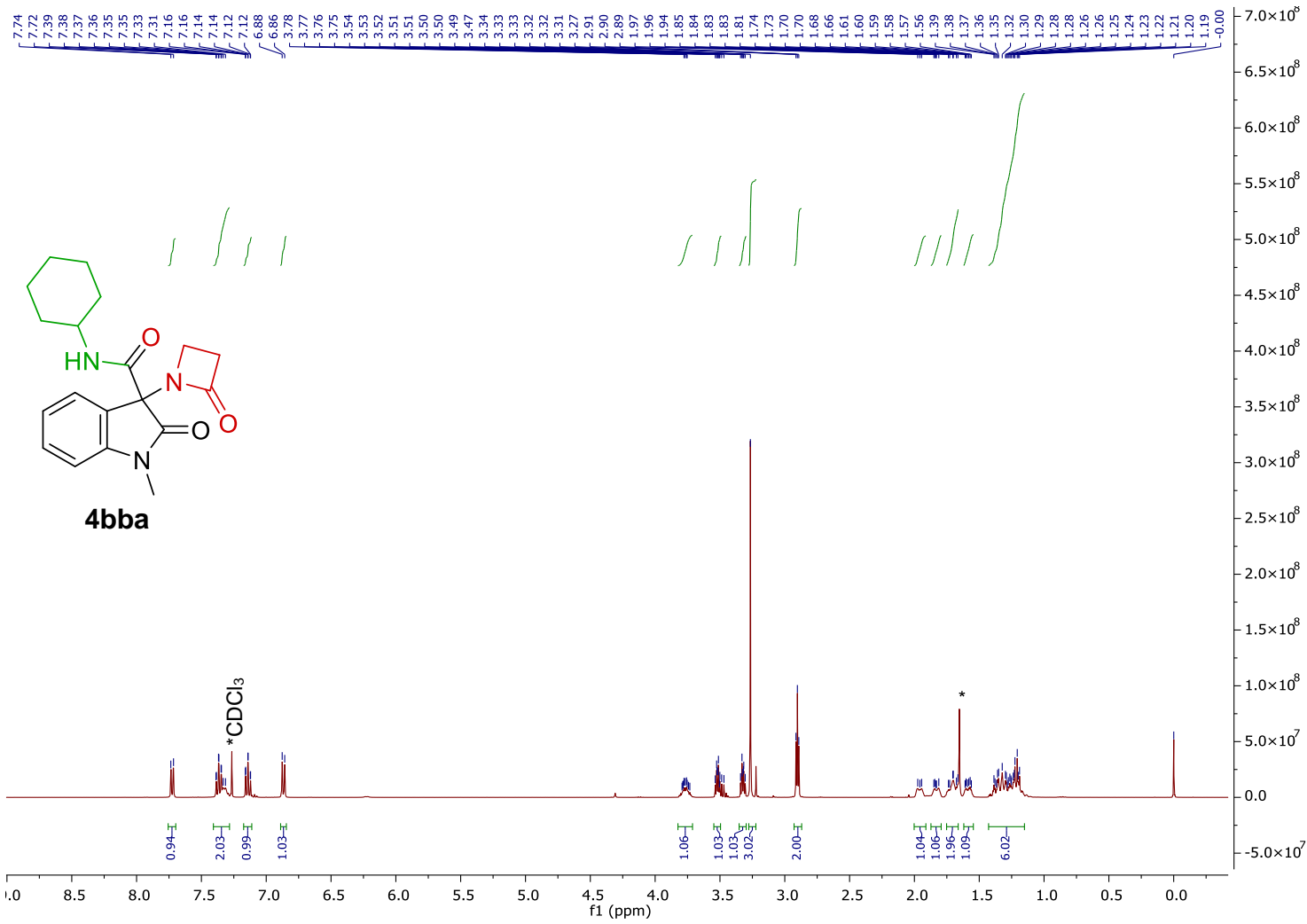

*acetone residue

${ }^{13} \mathrm{C}$ NMR Spectra of (4bba) (100 MHz, $\left.\mathrm{CDCl}_{3}\right)$ :

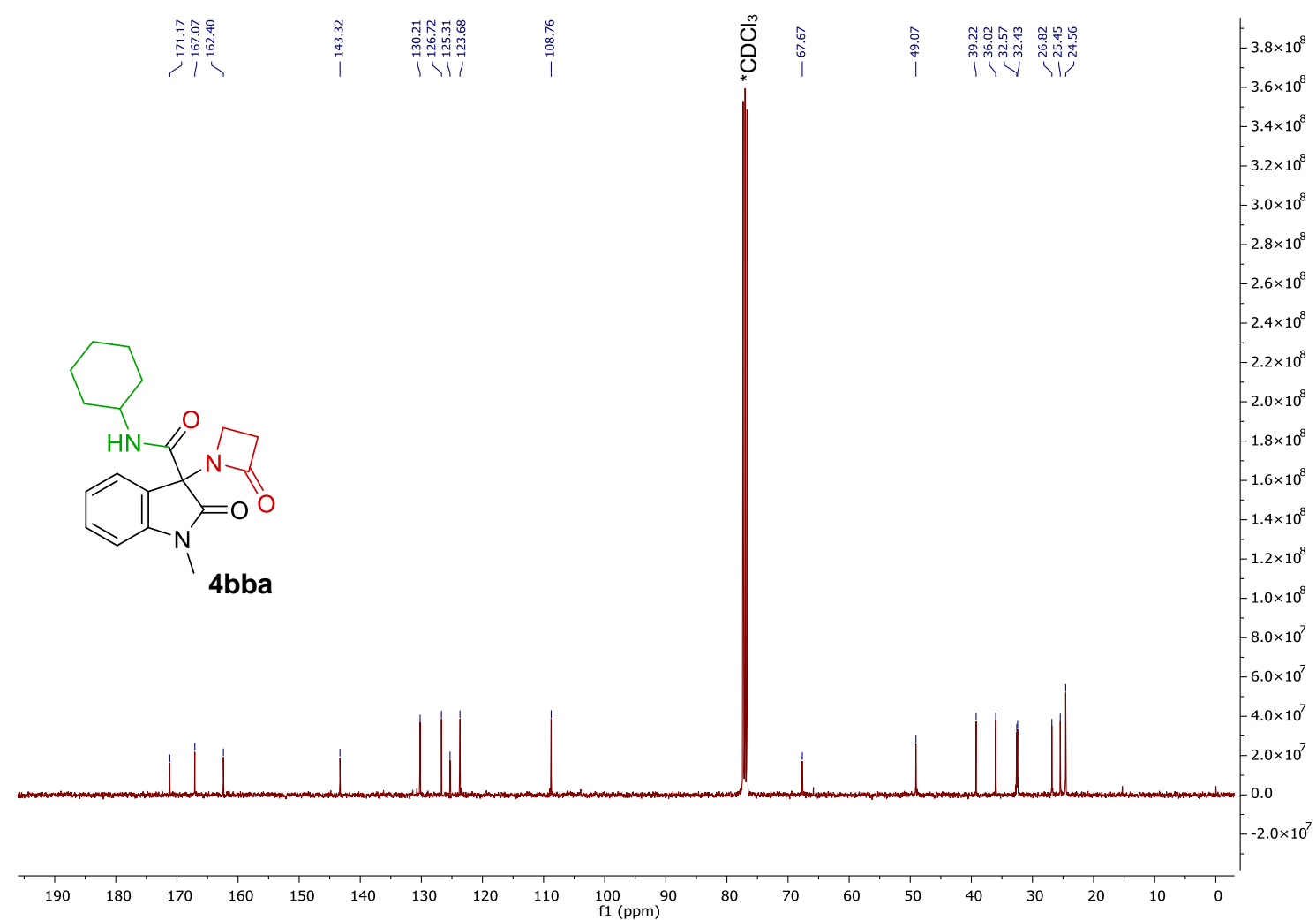


${ }^{1} \mathrm{H}$ NMR Spectra of (4bca) (400 MHz, $\left.\mathrm{CDCl}_{3}\right)$ :

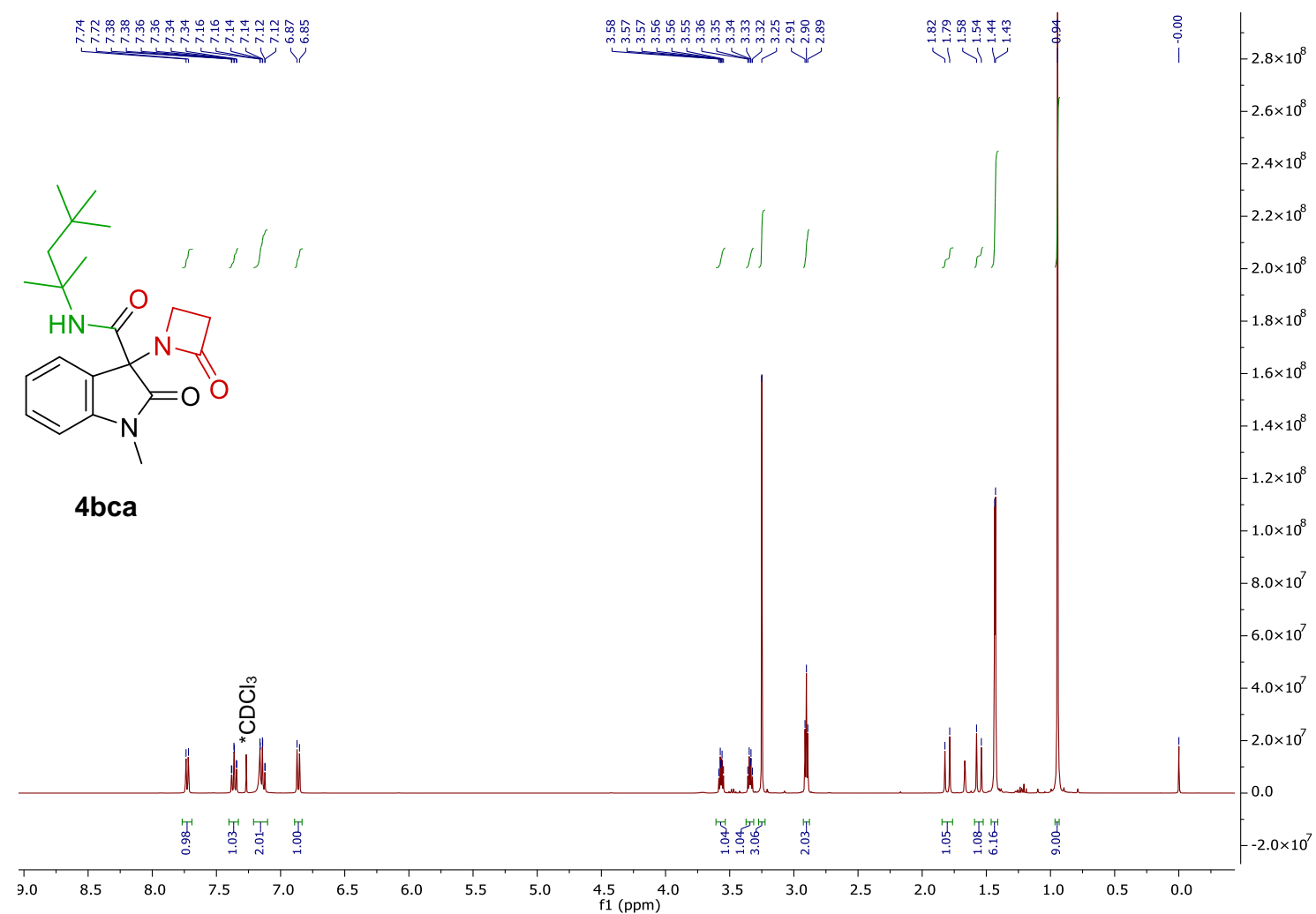

${ }^{13} \mathrm{C}$ NMR Spectra of (4bca) (100 MHz, $\mathrm{CDCl}_{3}$ ):

\section{号}

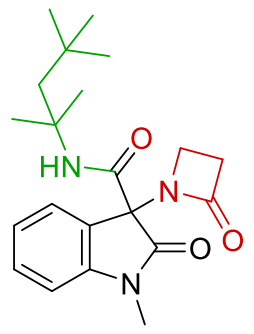

|

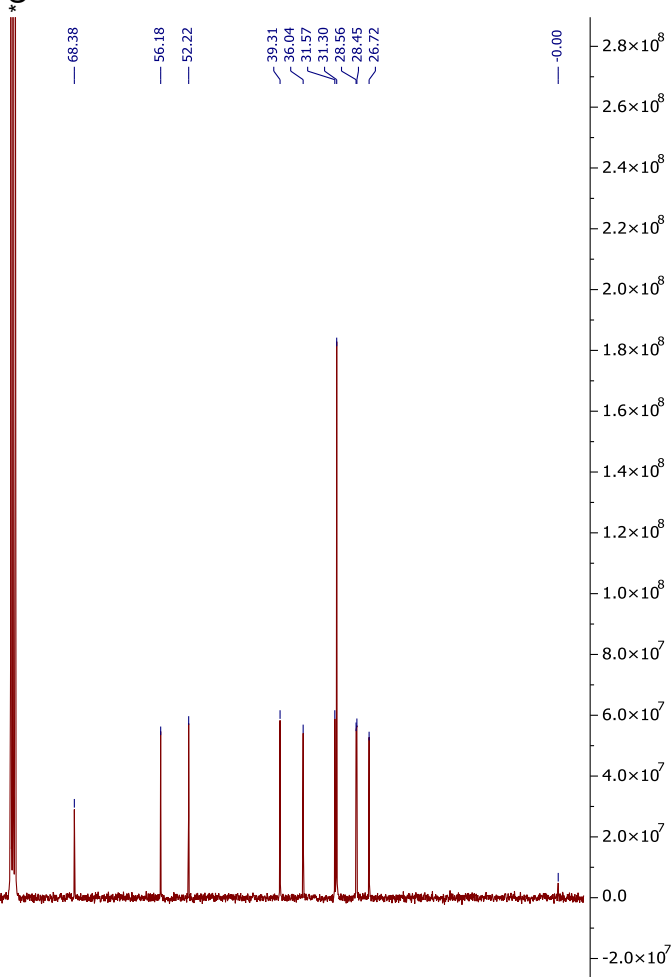

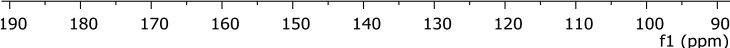

4 bca

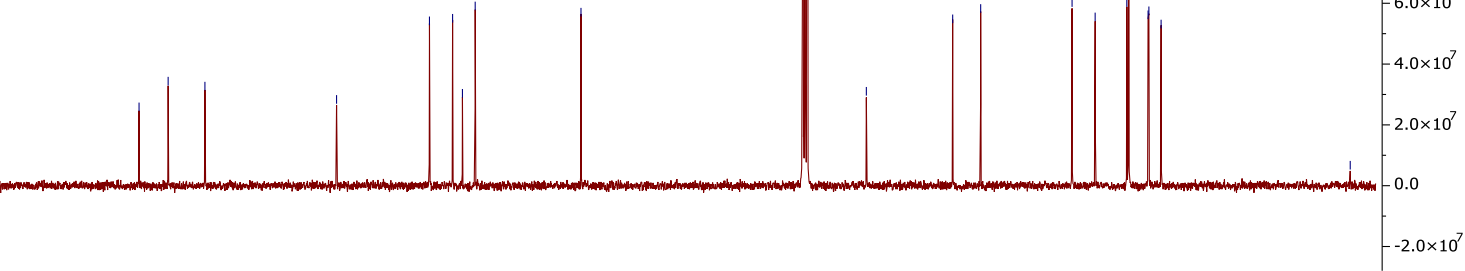


2.2. ${ }^{1} \mathrm{H}$ and ${ }^{13} \mathrm{C}$ NMR spectra of $\mathrm{y}$-lactam-oxindole hybrids

${ }^{1} \mathrm{H}$ NMR Spectra of (5aab) $\left(400 \mathrm{MHz}, \mathrm{CDCl}_{3}\right)$ :

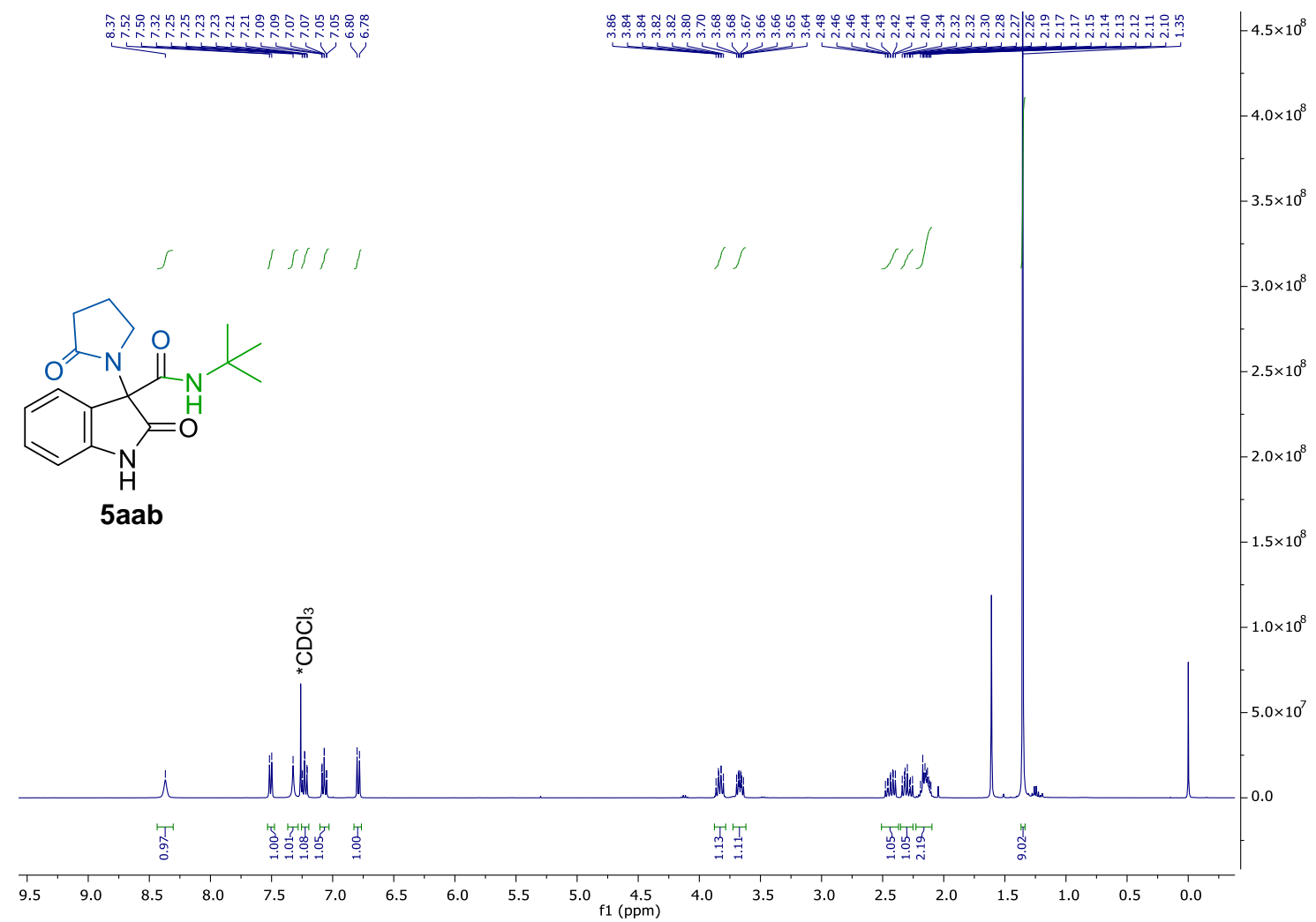

${ }^{13} \mathrm{C}$ NMR Spectra of (5aab) (100 MHz, $\left.\mathrm{CDCl}_{3}\right)$ :

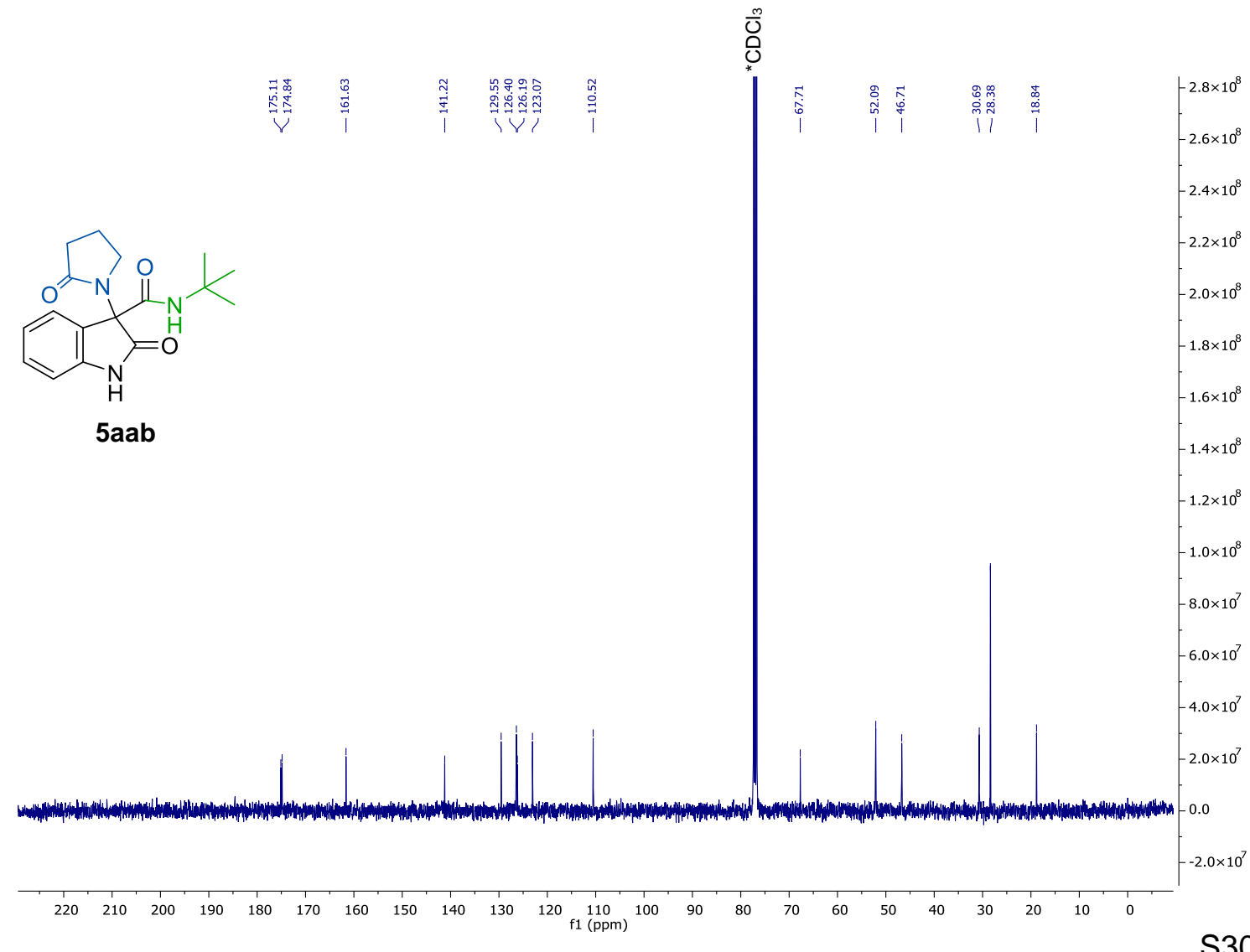


${ }^{1} \mathrm{H}$ NMR Spectra of (5bab) $\left(400 \mathrm{MHz}, \mathrm{CDCl}_{3}\right)$ :

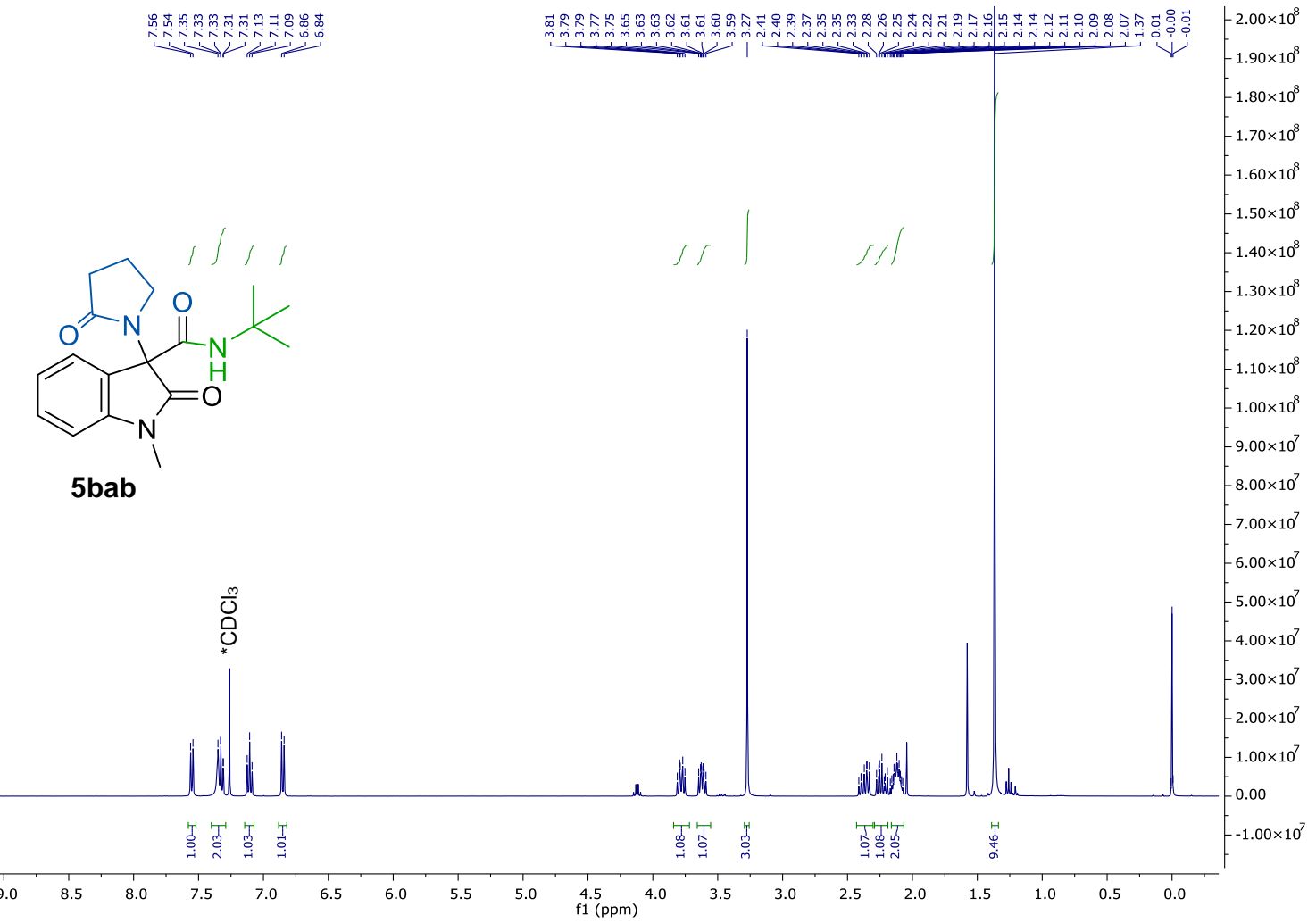

${ }^{13} \mathrm{C}$ NMR Spectra of (5bab) $\left(100 \mathrm{MHz}, \mathrm{CDCl}_{3}\right)$ :

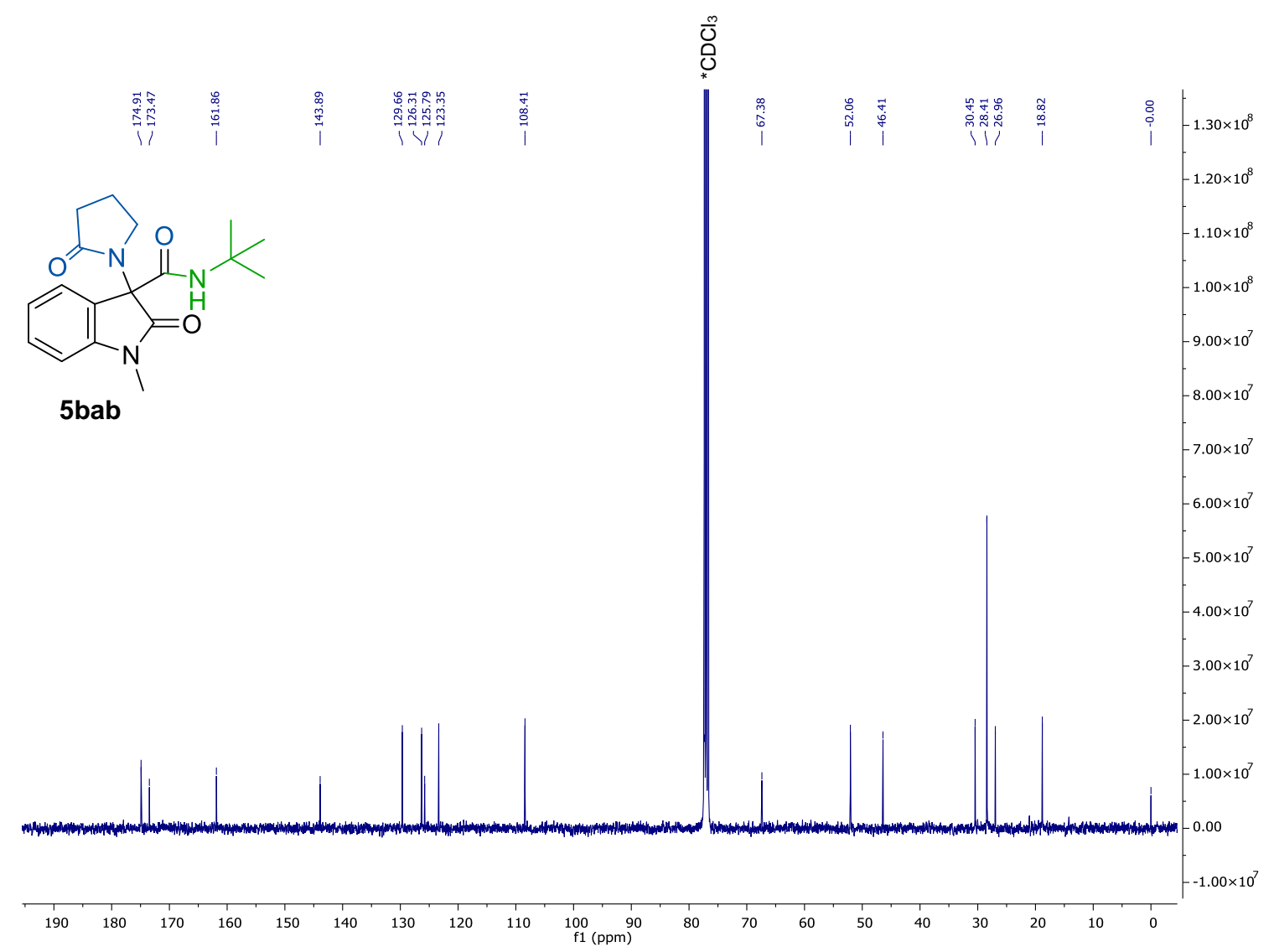


${ }^{1} \mathrm{H}$ NMR Spectra of (5dab) $\left(400 \mathrm{MHz}, \mathrm{CDCl}_{3}\right)$ :

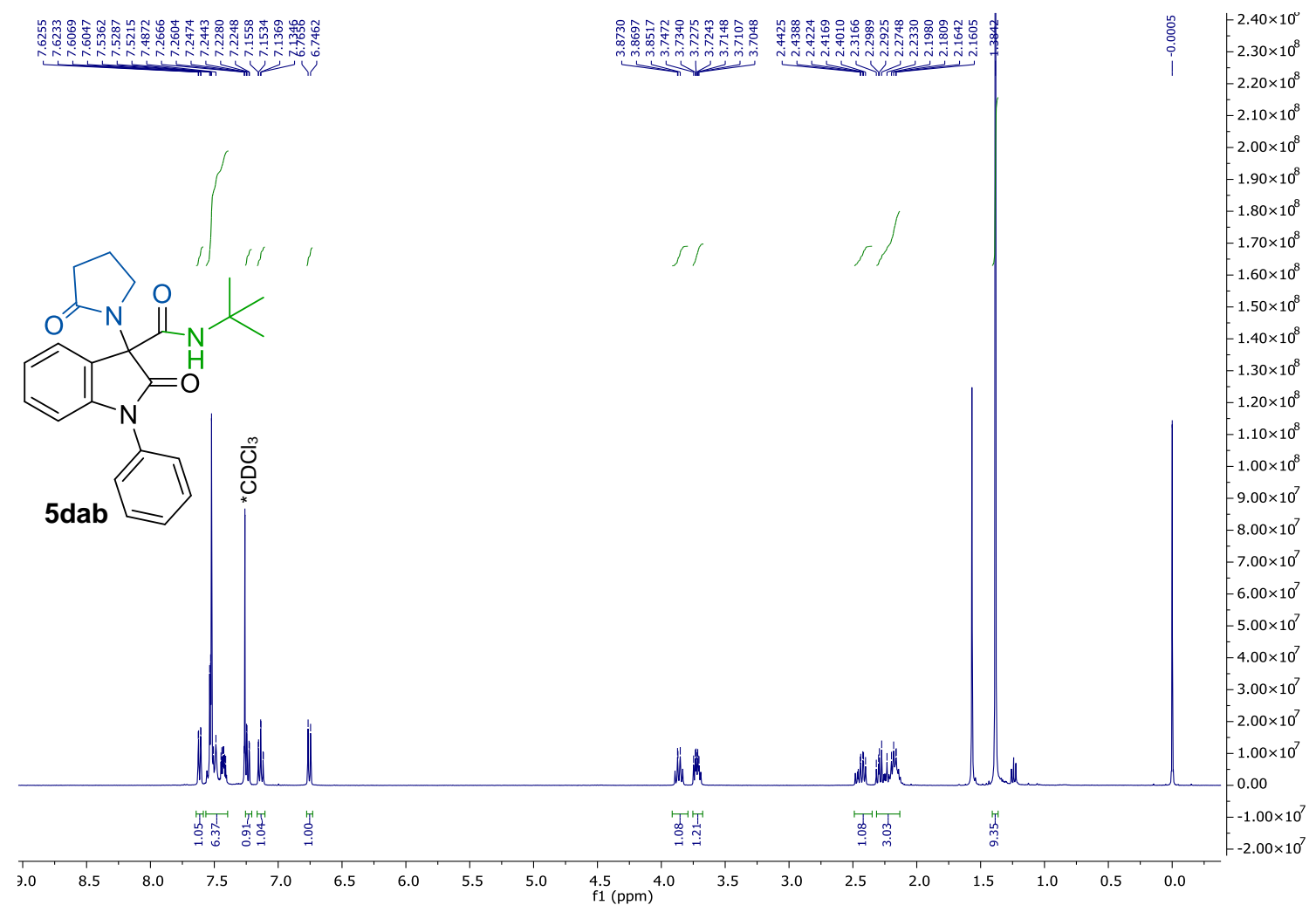

${ }^{13} \mathrm{C}$ NMR Spectra of (5dab) $\left(100 \mathrm{MHz}, \mathrm{CDCl}_{3}\right)$ :

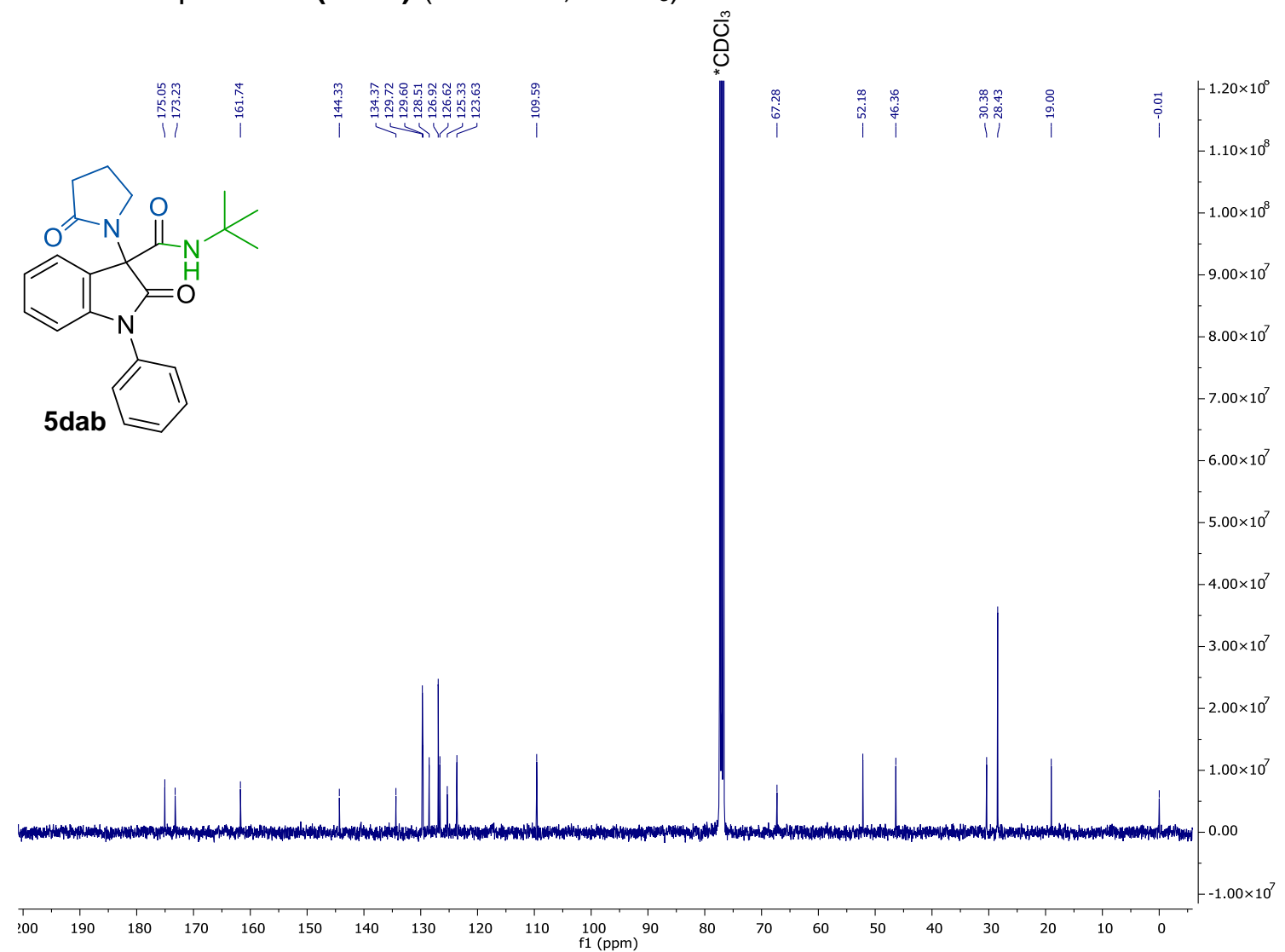


${ }^{1} \mathrm{H}$ NMR Spectra of (5eab) $\left(400 \mathrm{MHz}, \mathrm{CDCl}_{3}\right)$ :

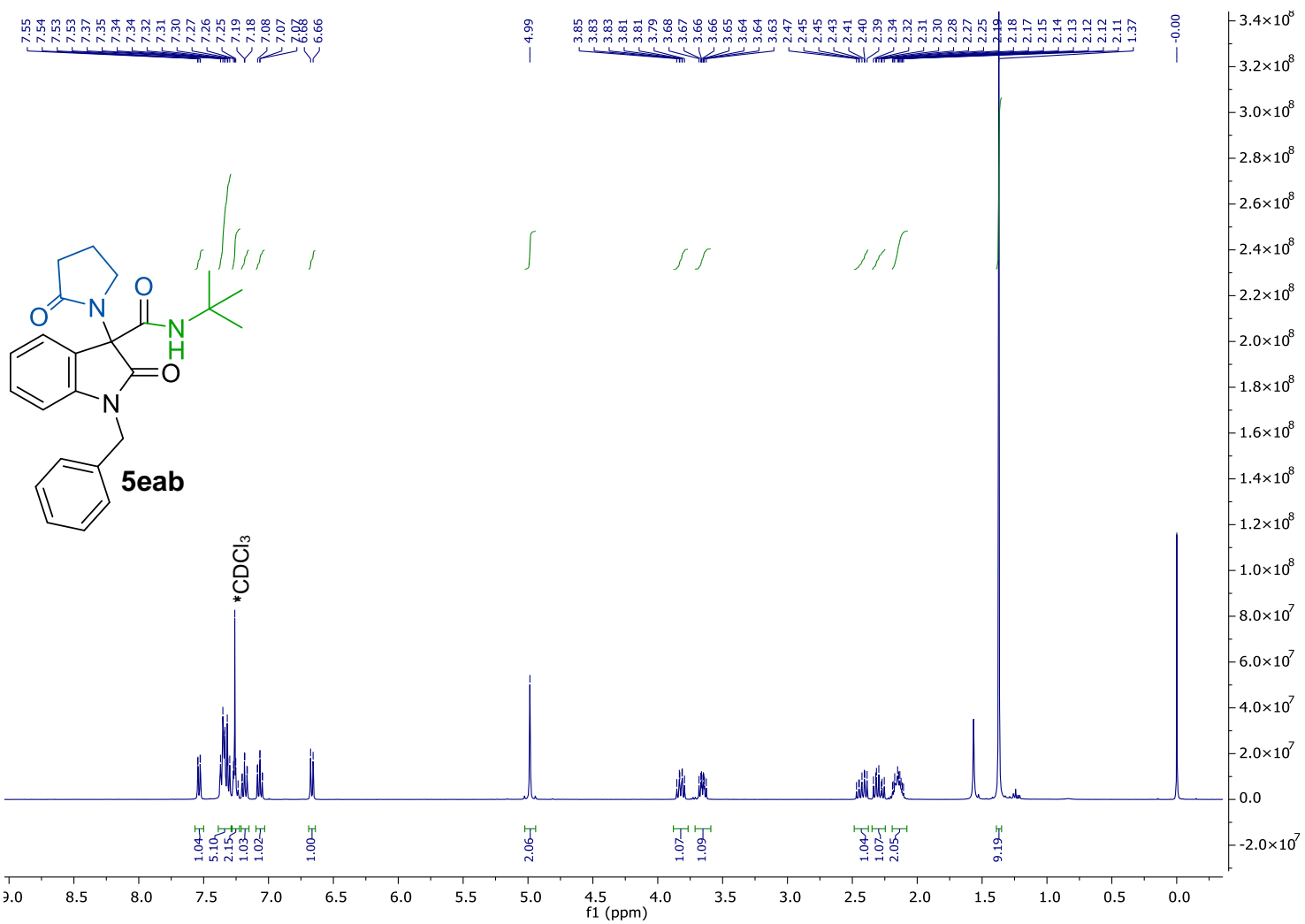

${ }^{13} \mathrm{C}$ NMR Spectra of (5eab) $\left(100 \mathrm{MHz}, \mathrm{CDCl}_{3}\right)$ :

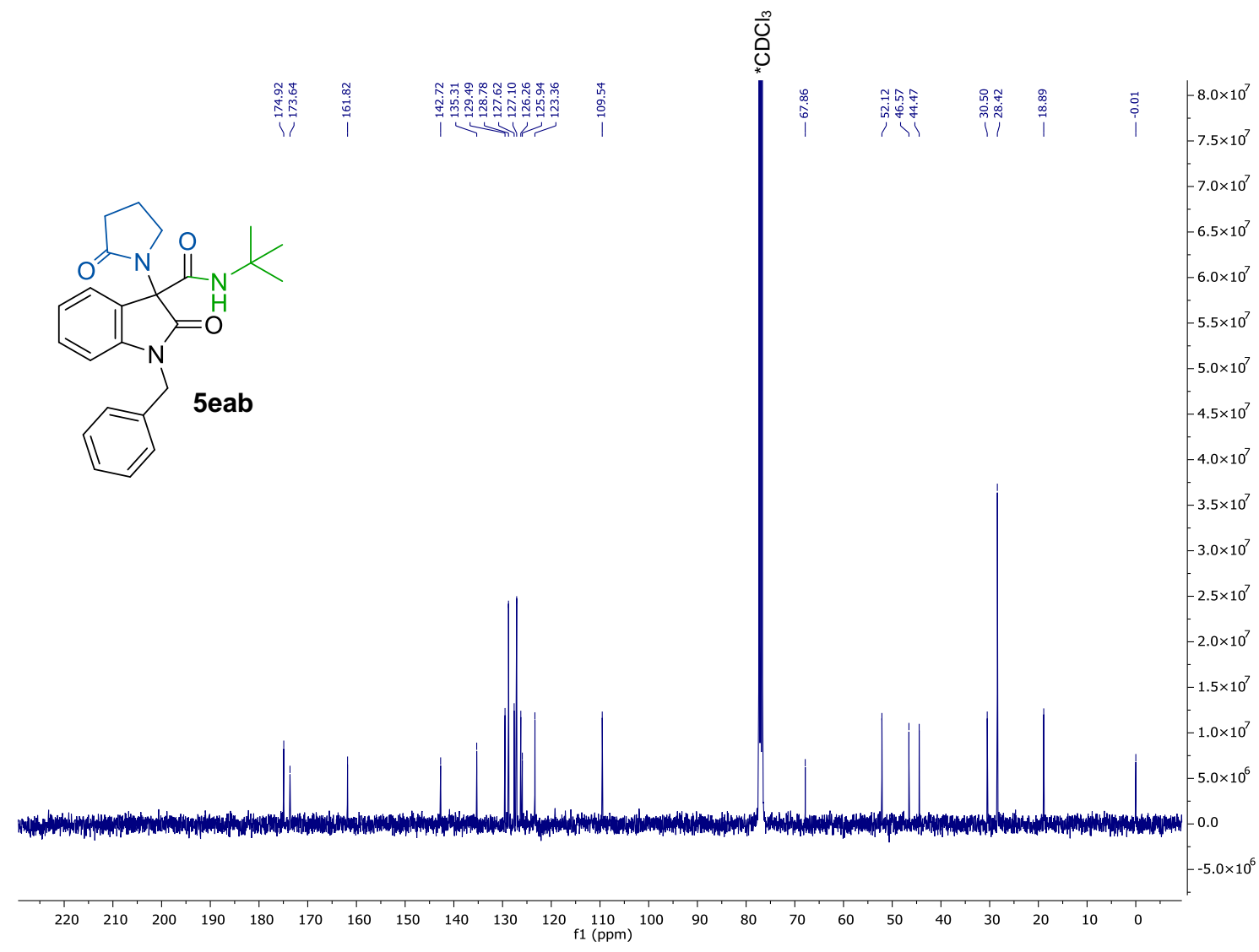


${ }^{1} \mathrm{H}$ NMR Spectra of (5fab) $\left(400 \mathrm{MHz}, \mathrm{CDCl}_{3}\right)$ :

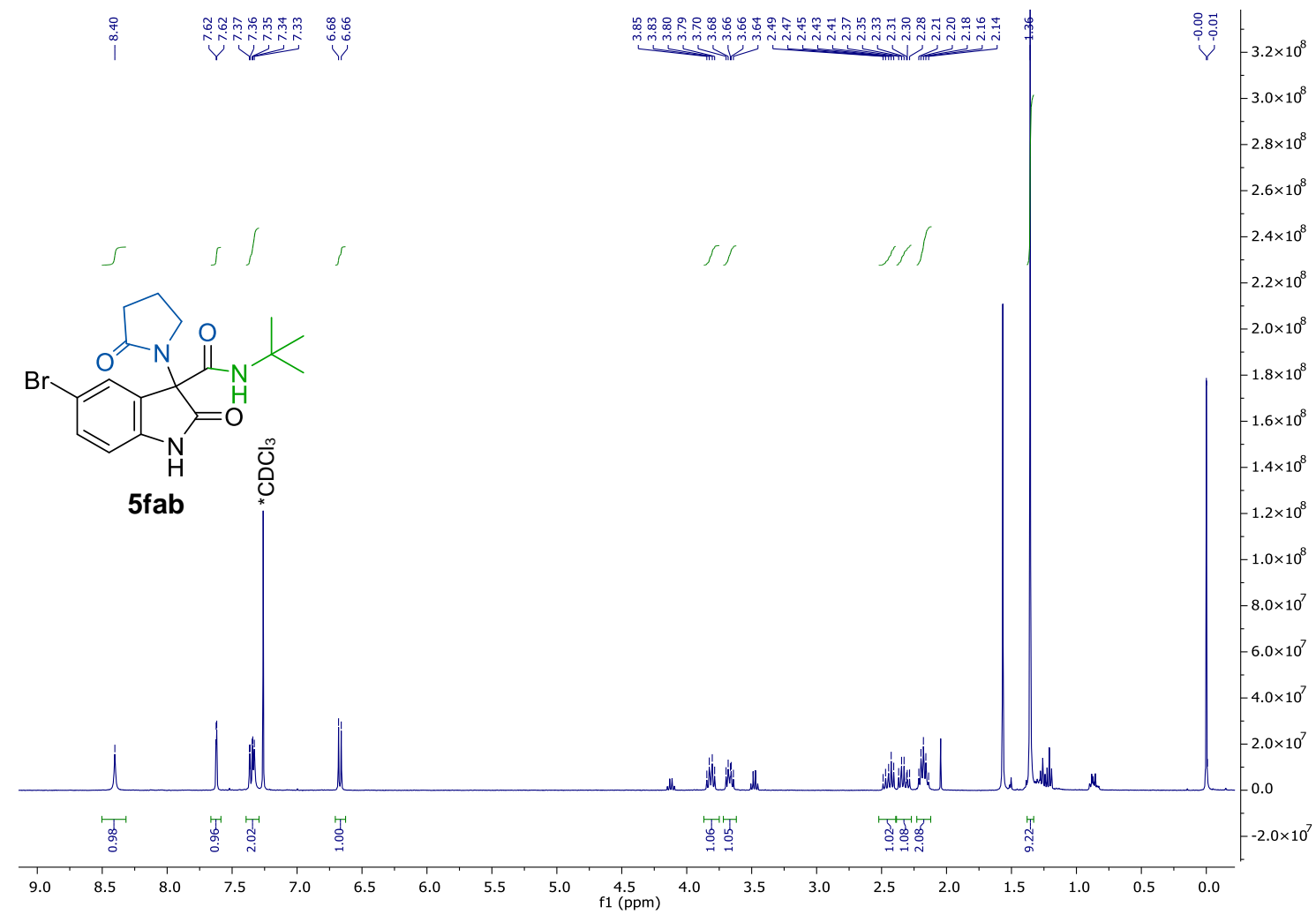

${ }^{13} \mathrm{C}$ NMR Spectra of (5fab) $\left(100 \mathrm{MHz}, \mathrm{CDCl}_{3}\right)$ :
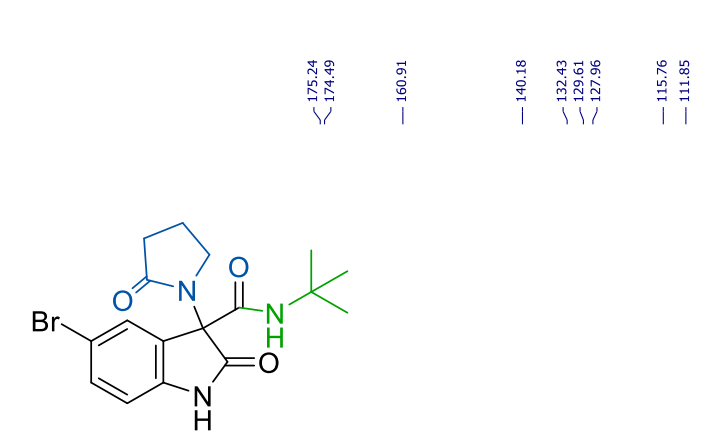

$5 \mathrm{fab}$

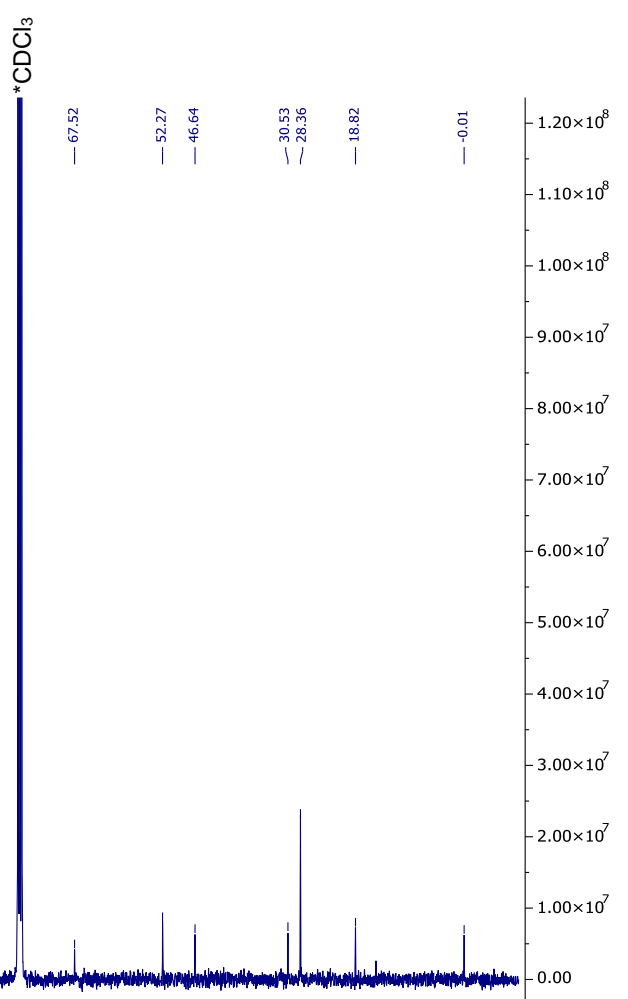

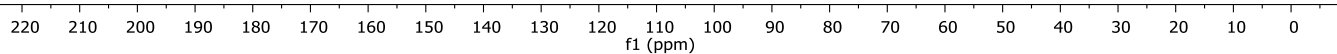

$-1.00 \times 10^{7}$ 
${ }^{1} \mathrm{H}$ NMR Spectra of (5gab) (400 MHz, $\left.\mathrm{CDCl}_{3}\right)$ :

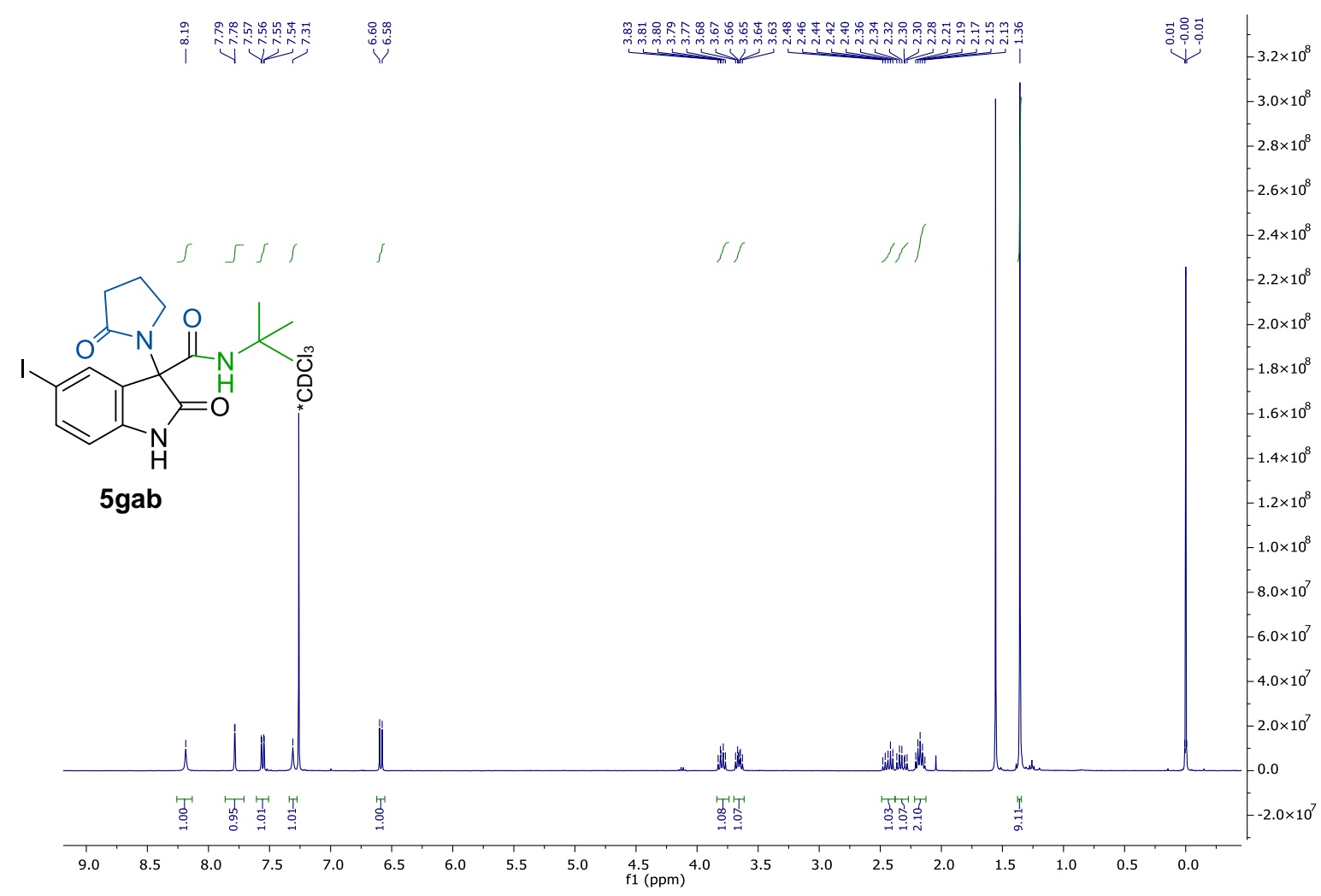

${ }^{13} \mathrm{C}$ NMR Spectra of (5gab) $\left(100 \mathrm{MHz}, \mathrm{CDCl}_{3}\right)$ :

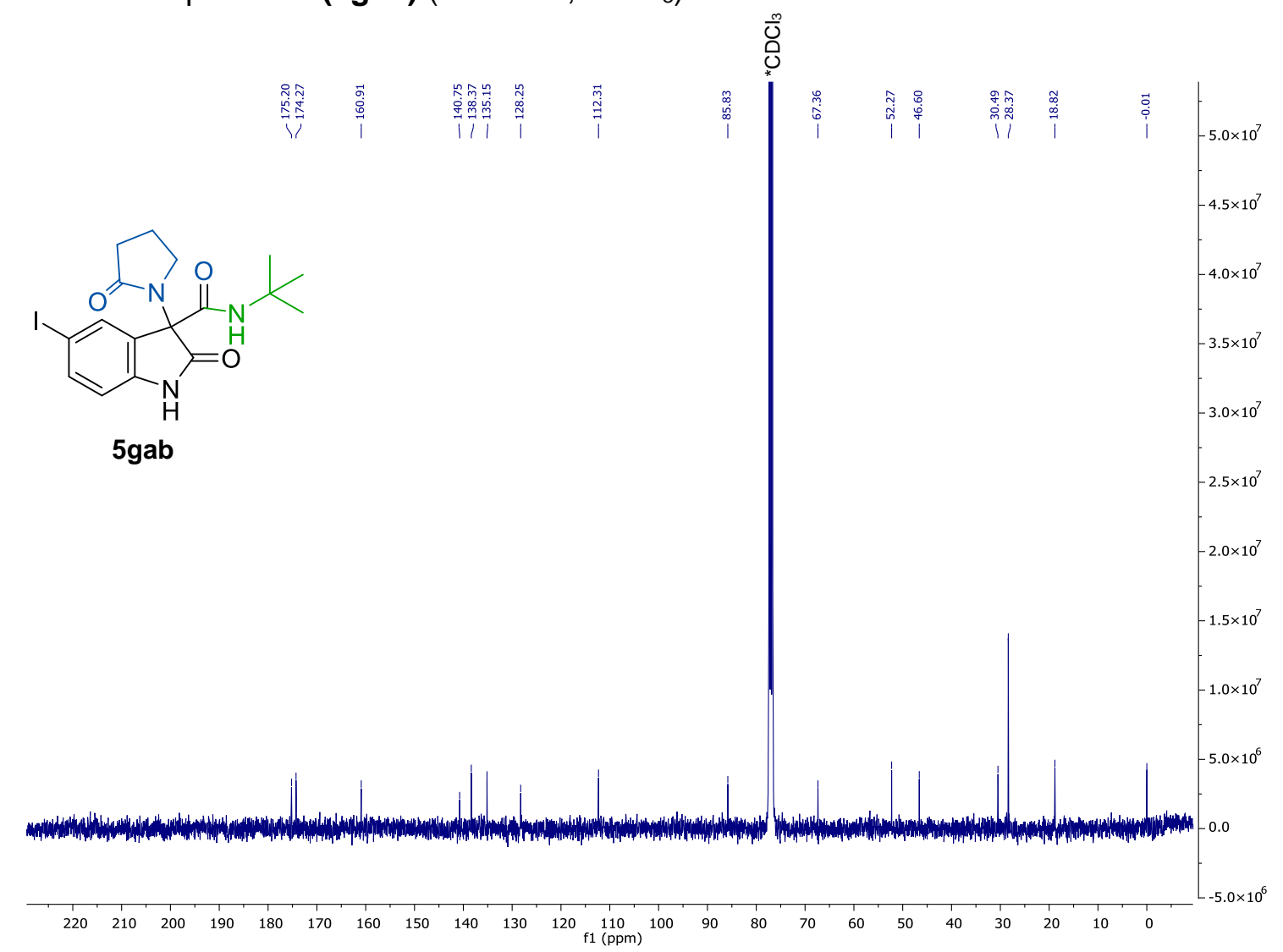


${ }^{1} \mathrm{H}$ NMR Spectra of (5hab) (400 MHz, $\left.\mathrm{CDCl}_{3}\right)$ :

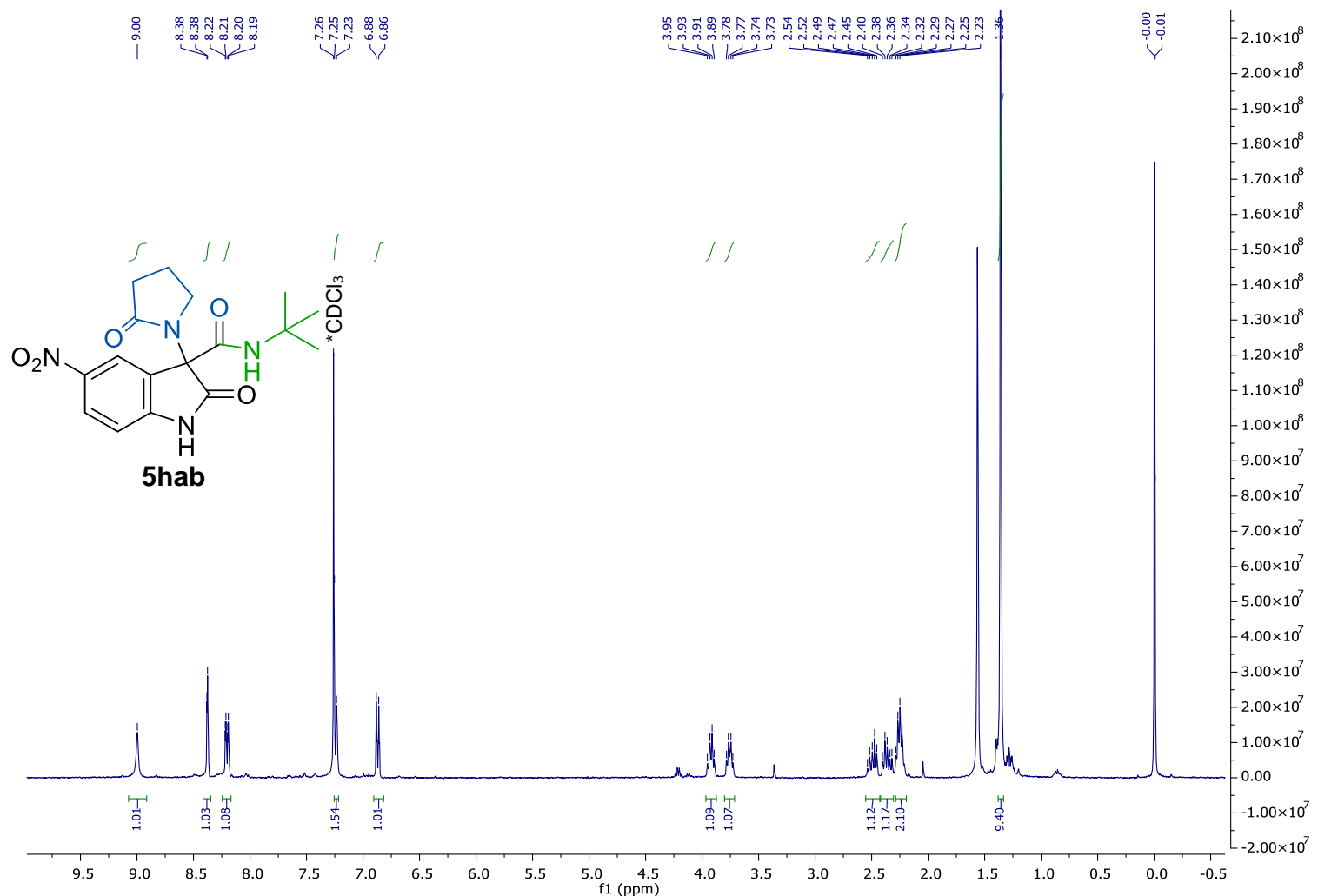

${ }^{13} \mathrm{C}$ NMR Spectra of (5hab) $\left(100 \mathrm{MHz}, \mathrm{CDCl}_{3}\right)$ :

迹<smiles>CC(C)(C)NC(=O)C1(N2CCCC2=O)C(=O)Nc2ccc([N+](=O)[O-])cc21</smiles>

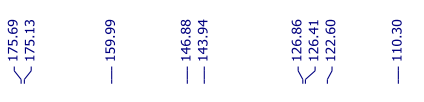

5hab

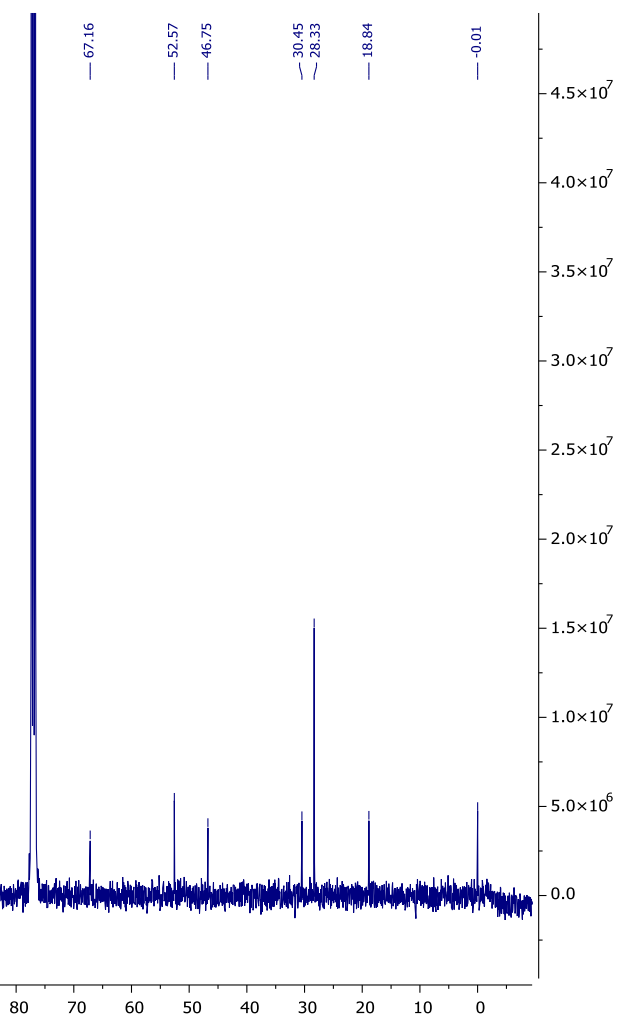


${ }^{1} \mathrm{H}$ NMR Spectra of (5iab) $\left(400 \mathrm{MHz}, \mathrm{CDCl}_{3}\right)$ :

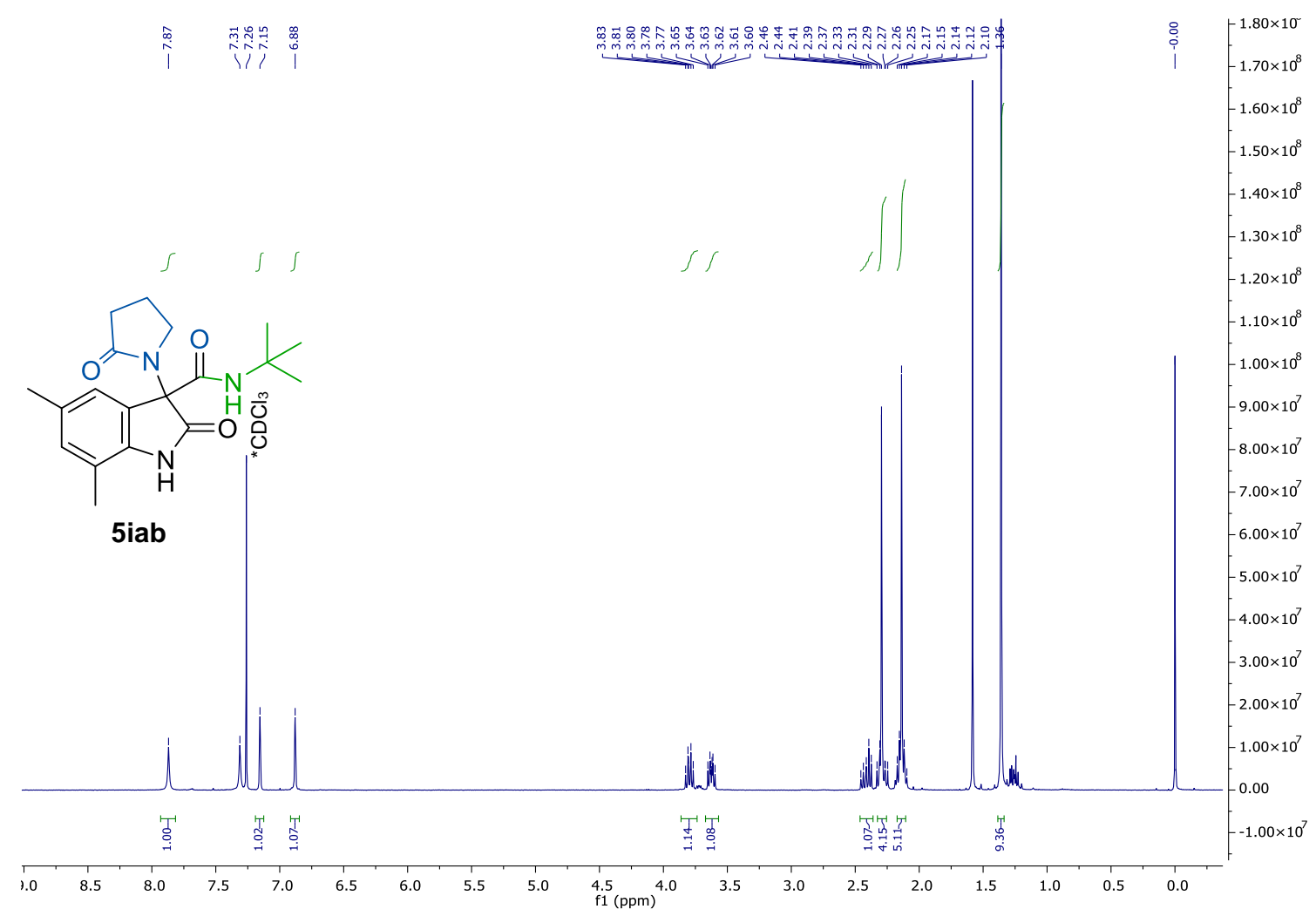

${ }^{13} \mathrm{C}$ NMR Spectra of (5iab) $\left(100 \mathrm{MHz}, \mathrm{CDCl}_{3}\right)$ :

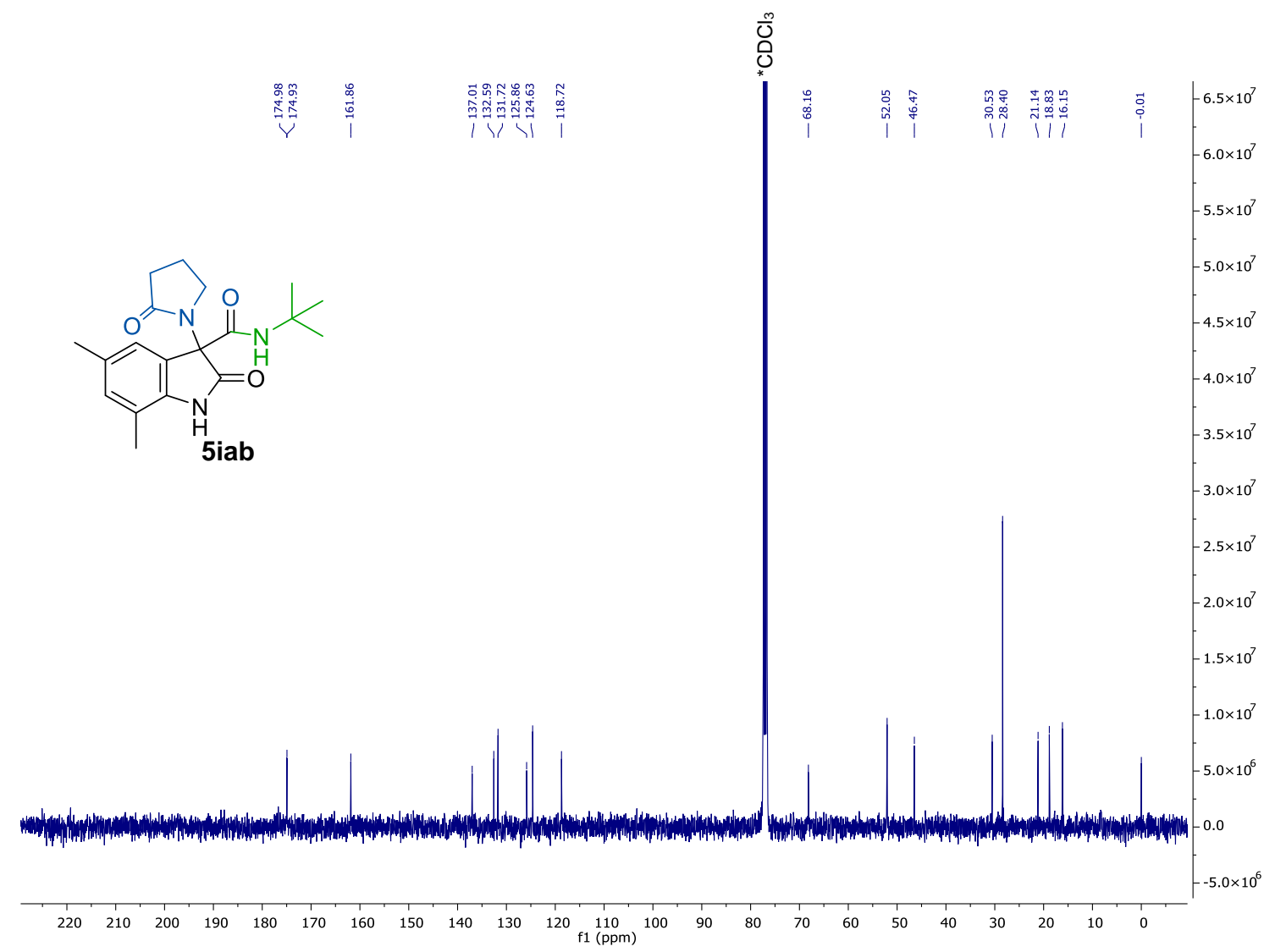


${ }^{1} \mathrm{H}$ NMR Spectra of (5jab) $\left(400 \mathrm{MHz}, \mathrm{CDCl}_{3}\right)$ :

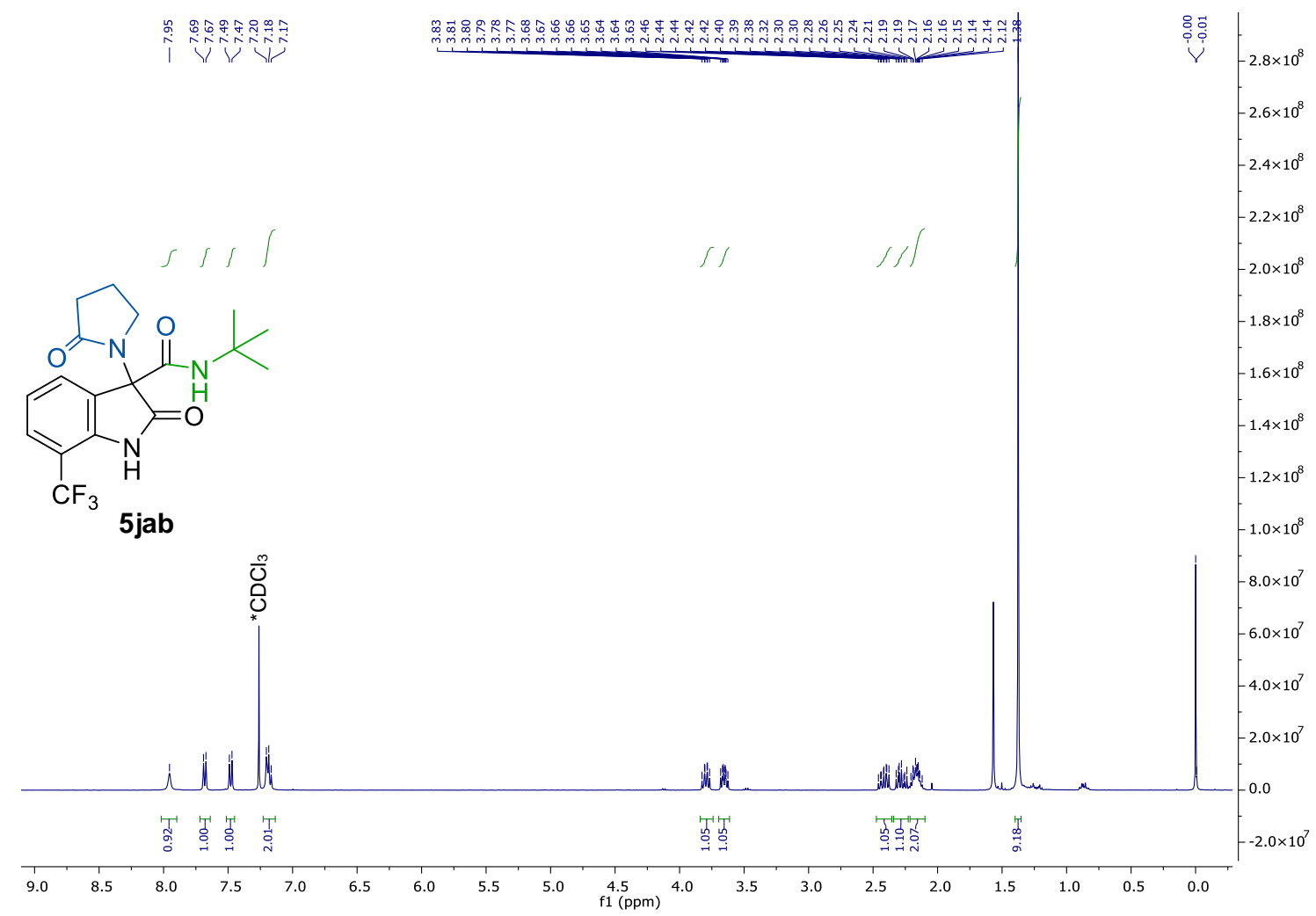

${ }^{13} \mathrm{C}$ NMR Spectra of (5jab) $\left(100 \mathrm{MHz}, \mathrm{CDCl}_{3}\right)$ :

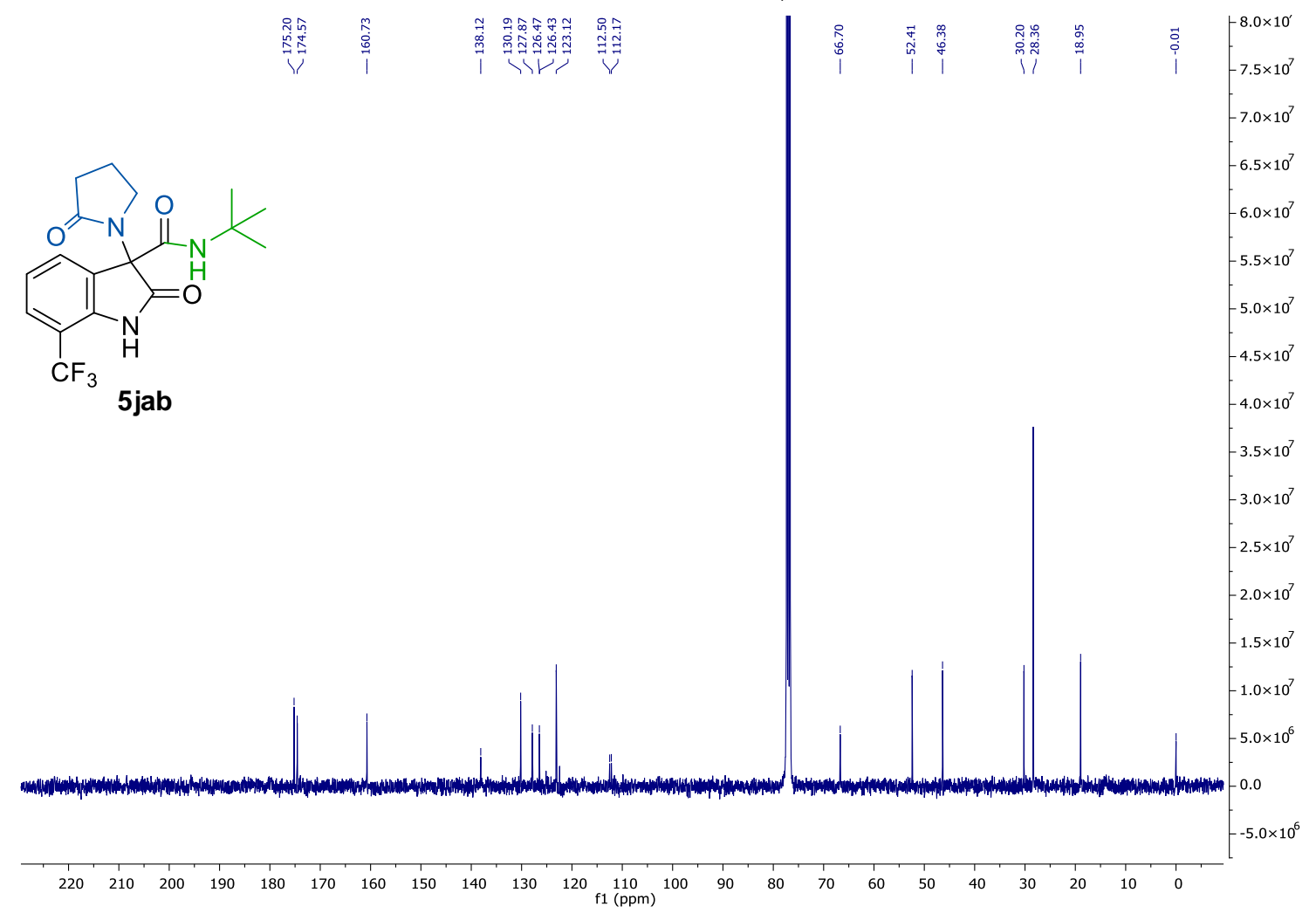

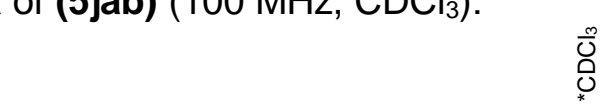


${ }^{1} \mathrm{H}$ NMR Spectra of (5abb) (400 MHz, $\left.\mathrm{CDCl}_{3}\right)$ :
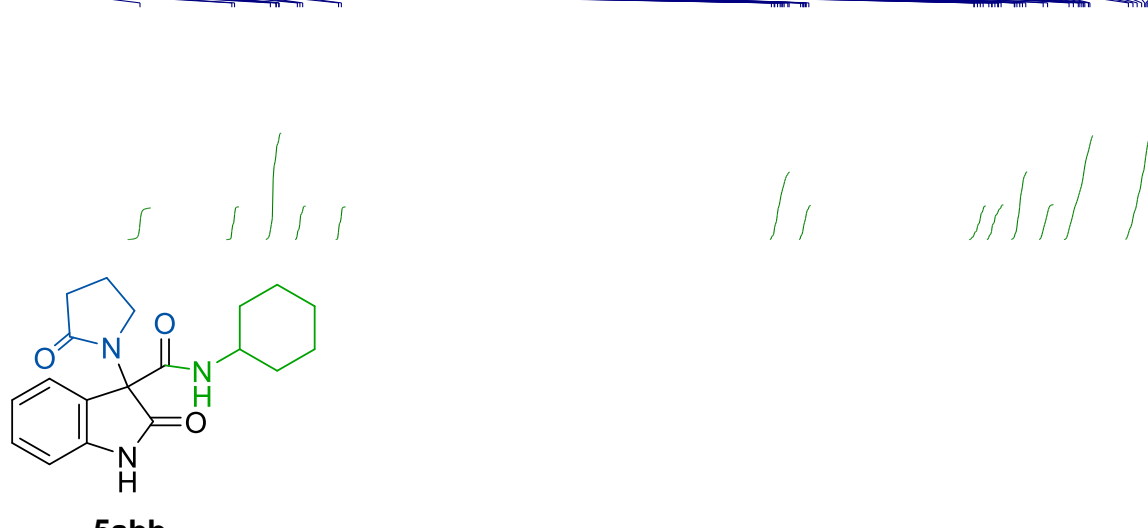

$1.50 \times 10^{9}$

$5 a b b$

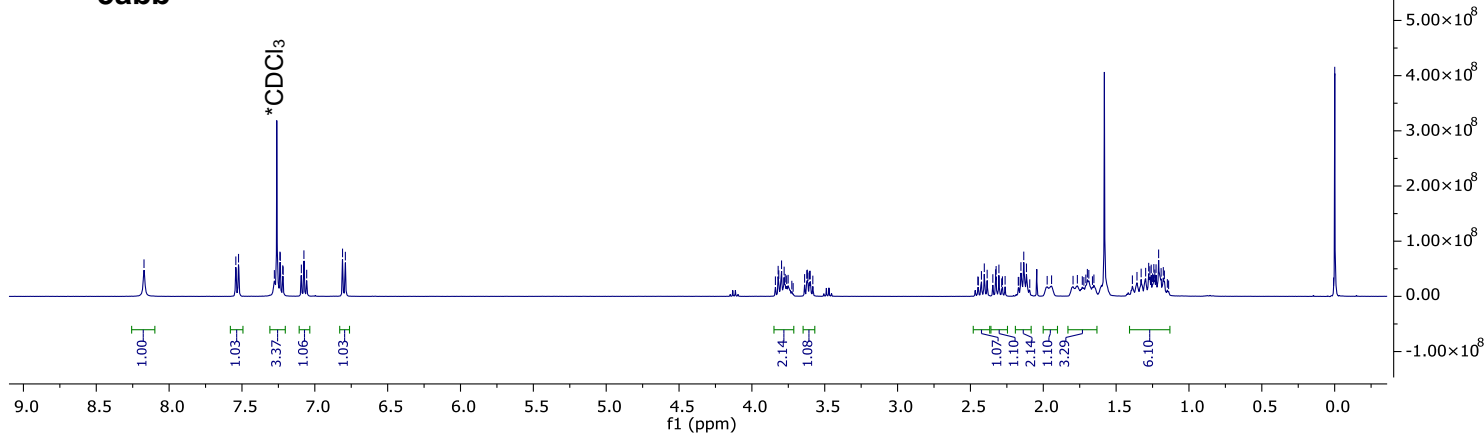

${ }^{13} \mathrm{C}$ NMR Spectra of (5abb) $\left(100 \mathrm{MHz}, \mathrm{CDCl}_{3}\right)$ :

足<smiles>O=C1Nc2ccccc2C1(C(=O)NC1CCCCC1)N1CCCC1</smiles>

$5 a b b$

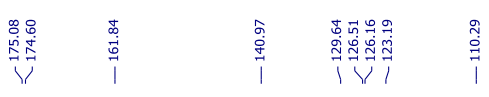

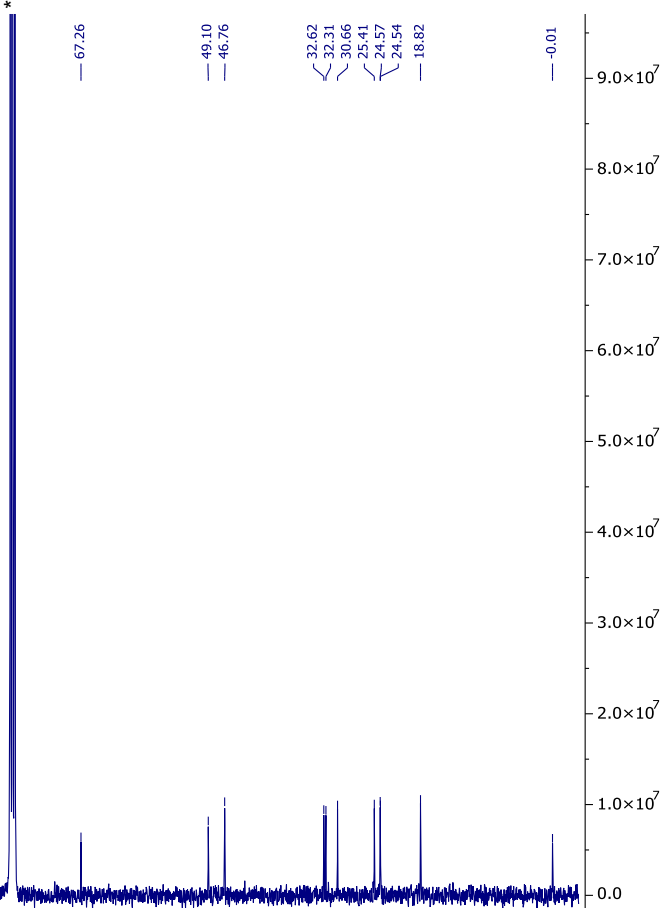

$\begin{array}{llllllllll}190 & 180 & 170 & 160 & 150 & 140 & 130 & 120 & 110 & 100 \\ f 1(\mathrm{ppm})\end{array}$ 
${ }^{1} \mathrm{H}$ NMR Spectra of (5acb) (400 MHz, $\left.\mathrm{CDCl}_{3}\right)$ :

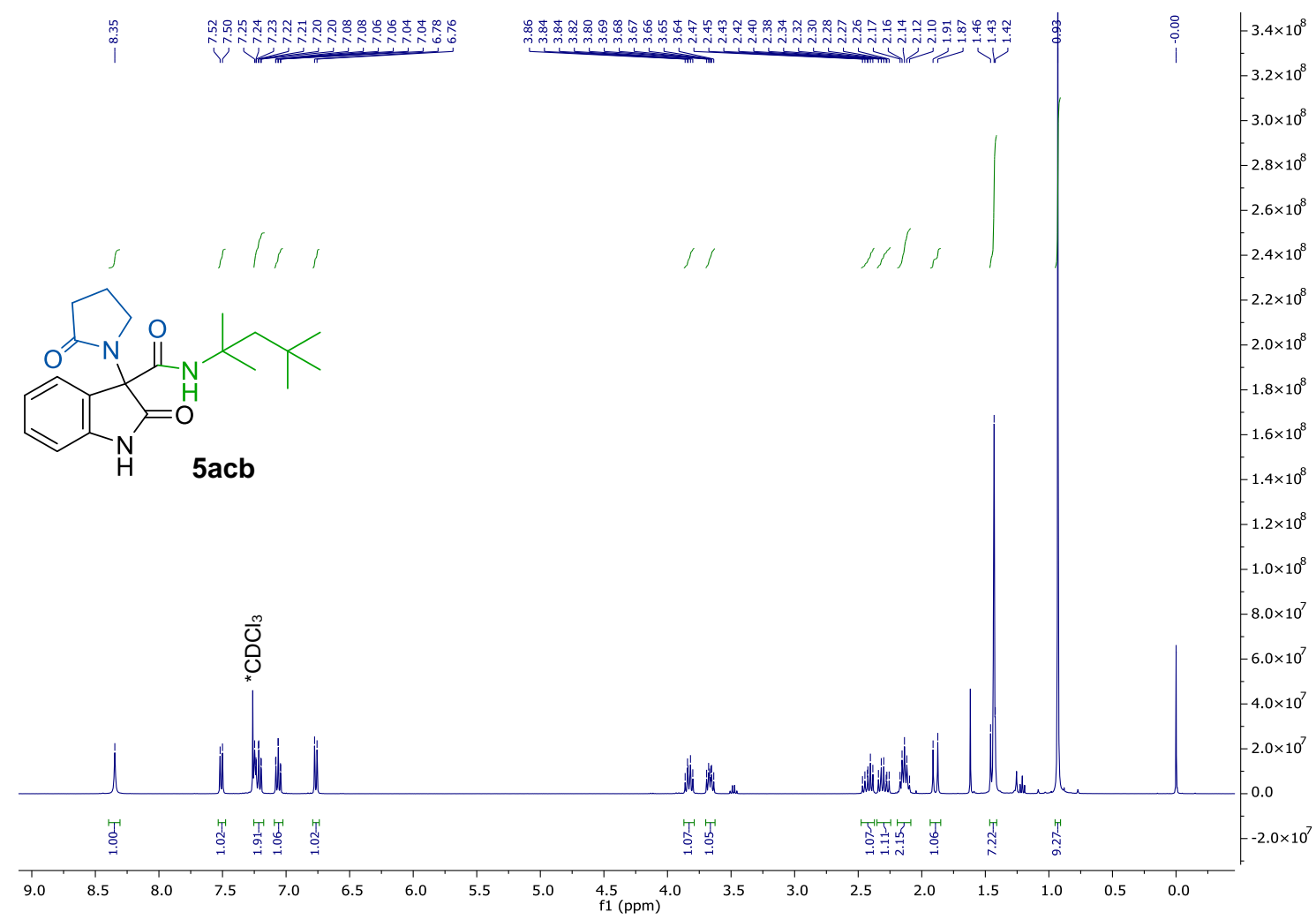

${ }^{13} \mathrm{C}$ NMR Spectra of (5acb) (100 MHz, $\left.\mathrm{CDCl}_{3}\right)$ :

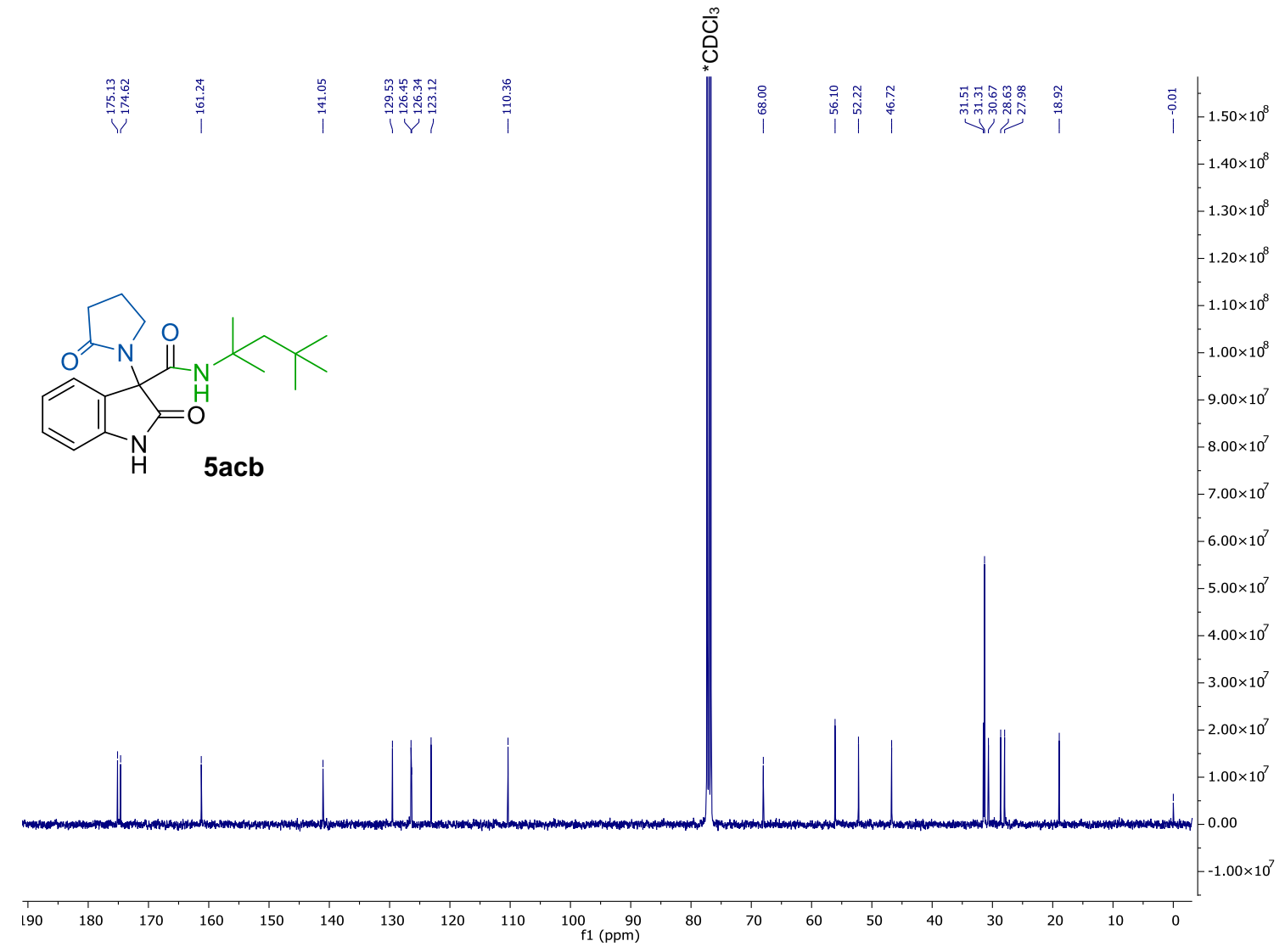


${ }^{1} \mathrm{H}$ NMR Spectra of (5adb) $\left(400 \mathrm{MHz}, \mathrm{CDCl}_{3}\right)$ :

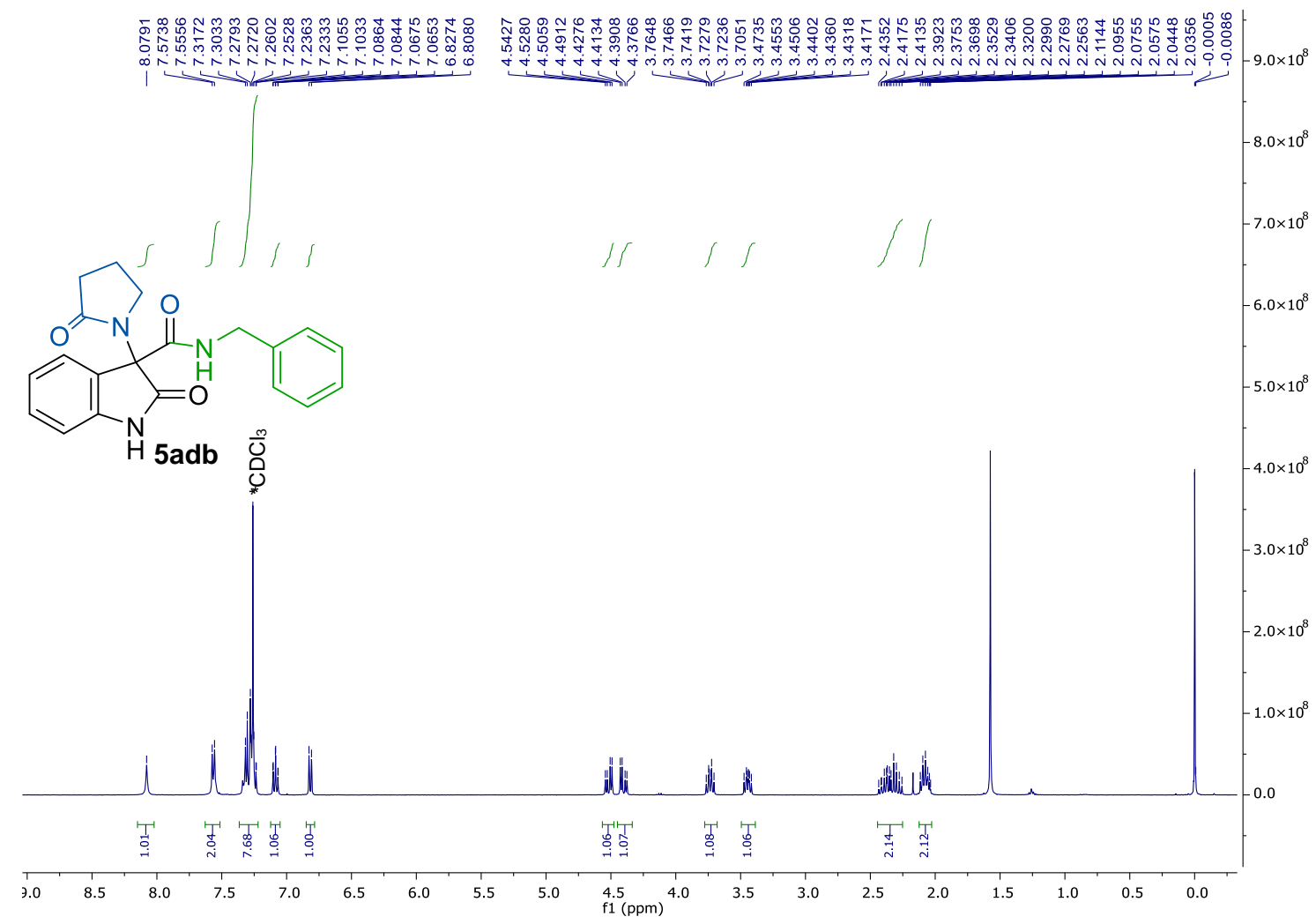

${ }^{13} \mathrm{C}$ NMR Spectra of (5adb) $\left(100 \mathrm{MHz}, \mathrm{CDCl}_{3}\right)$ :

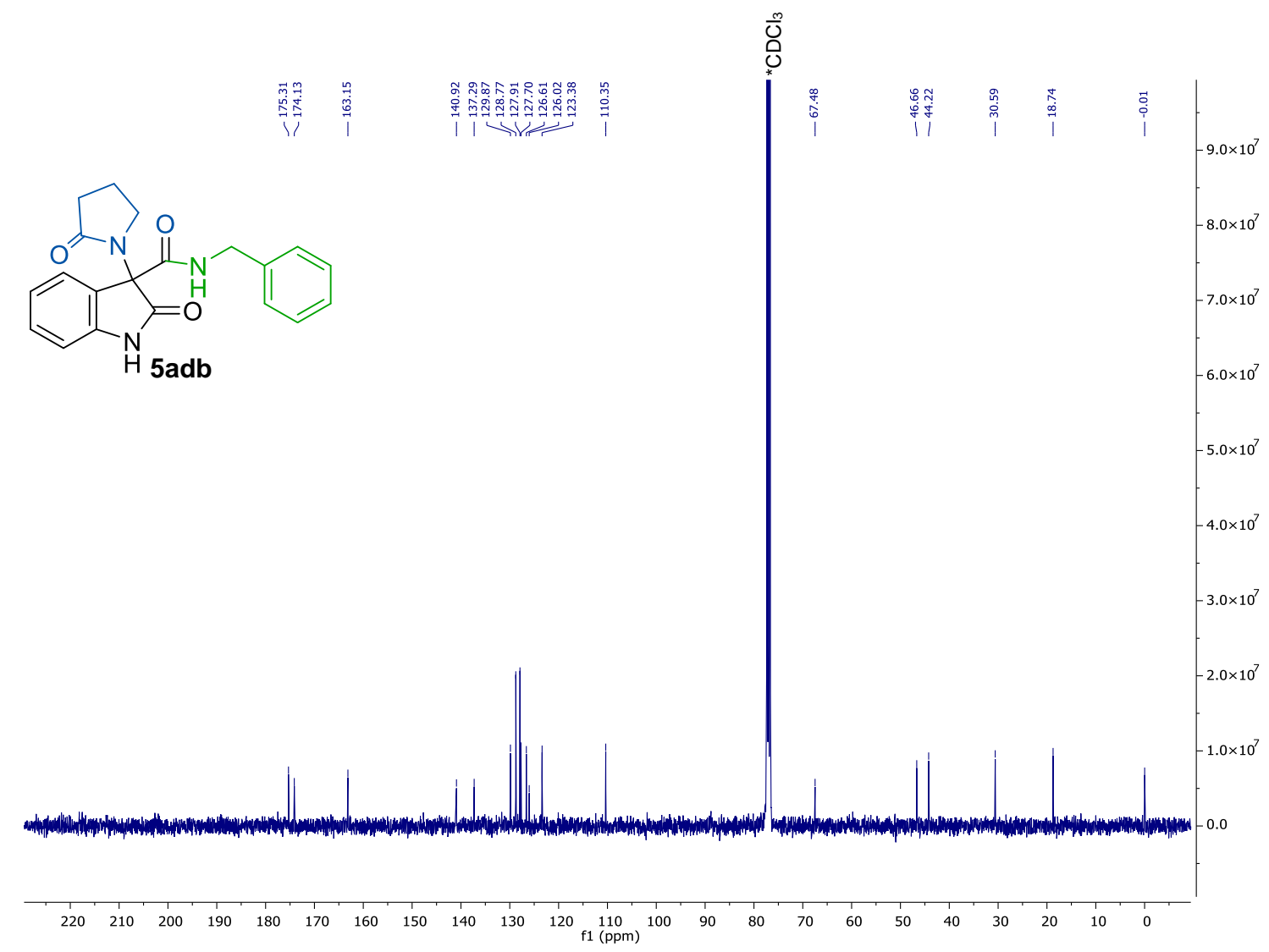


${ }^{1} \mathrm{H}$ NMR Spectra of (5bcb) (400 MHz, $\left.\mathrm{CDCl}_{3}\right)$ :

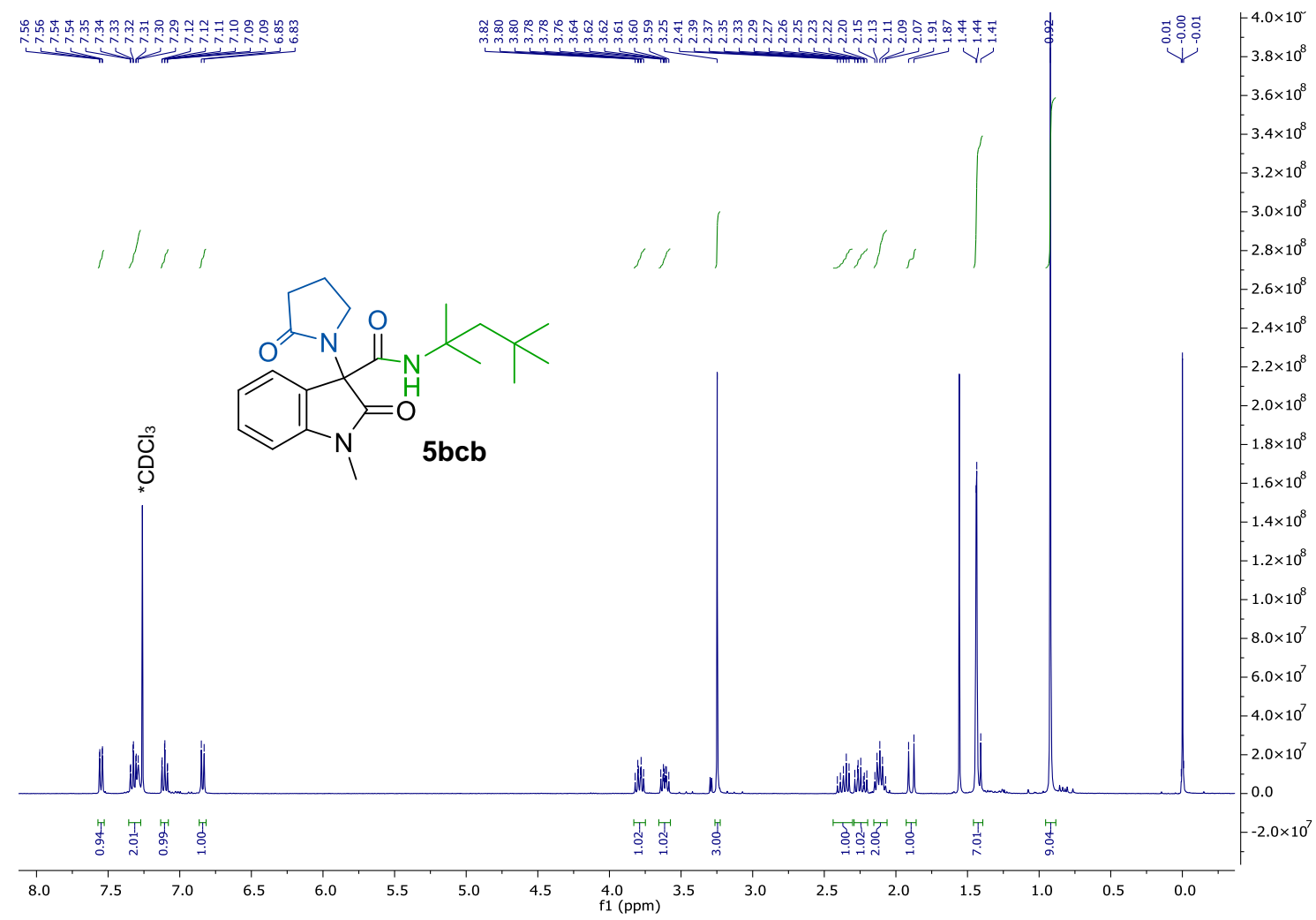

${ }^{13} \mathrm{C}$ NMR Spectra of (5bcb) $\left(100 \mathrm{MHz}, \mathrm{CDCl}_{3}\right)$ :

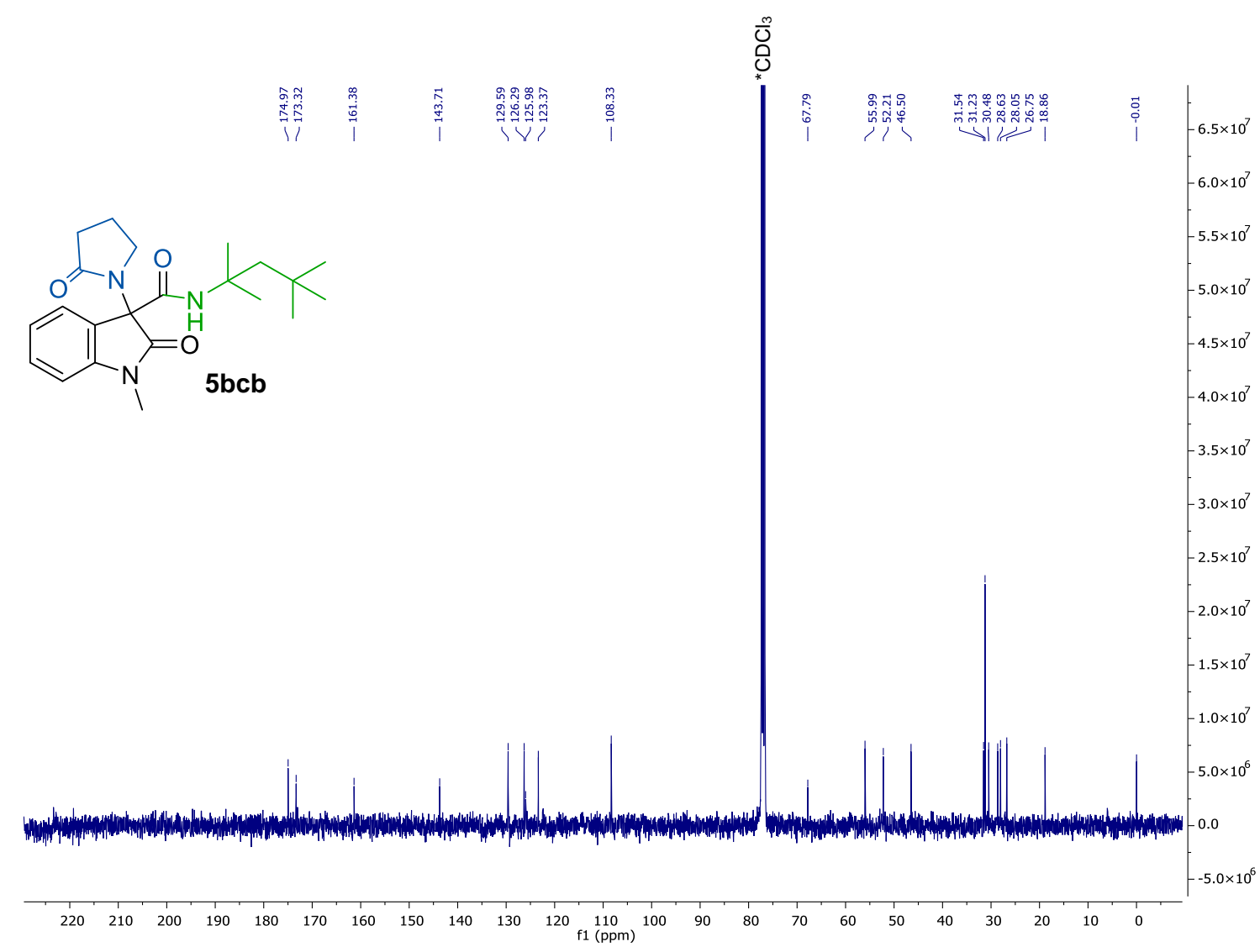


${ }^{1} \mathrm{H}$ NMR Spectra of $(\mathbf{5} \mathbf{c d b})\left(400 \mathrm{MHz}, \mathrm{CDCl}_{3}\right)$ :

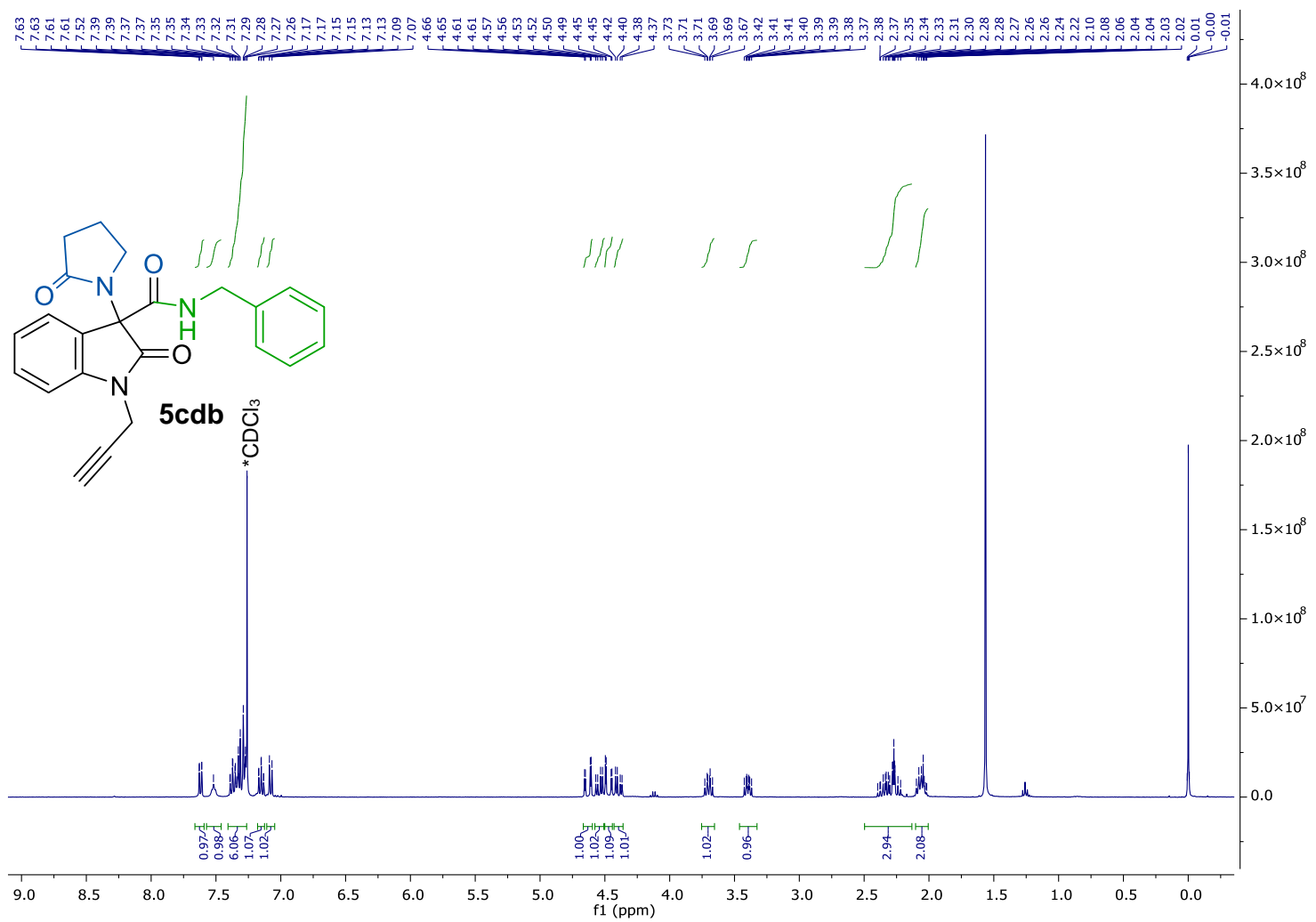

${ }^{13} \mathrm{C}$ NMR Spectra of $(\mathbf{5 c d b})\left(100 \mathrm{MHz}, \mathrm{CDCl}_{3}\right)$ :

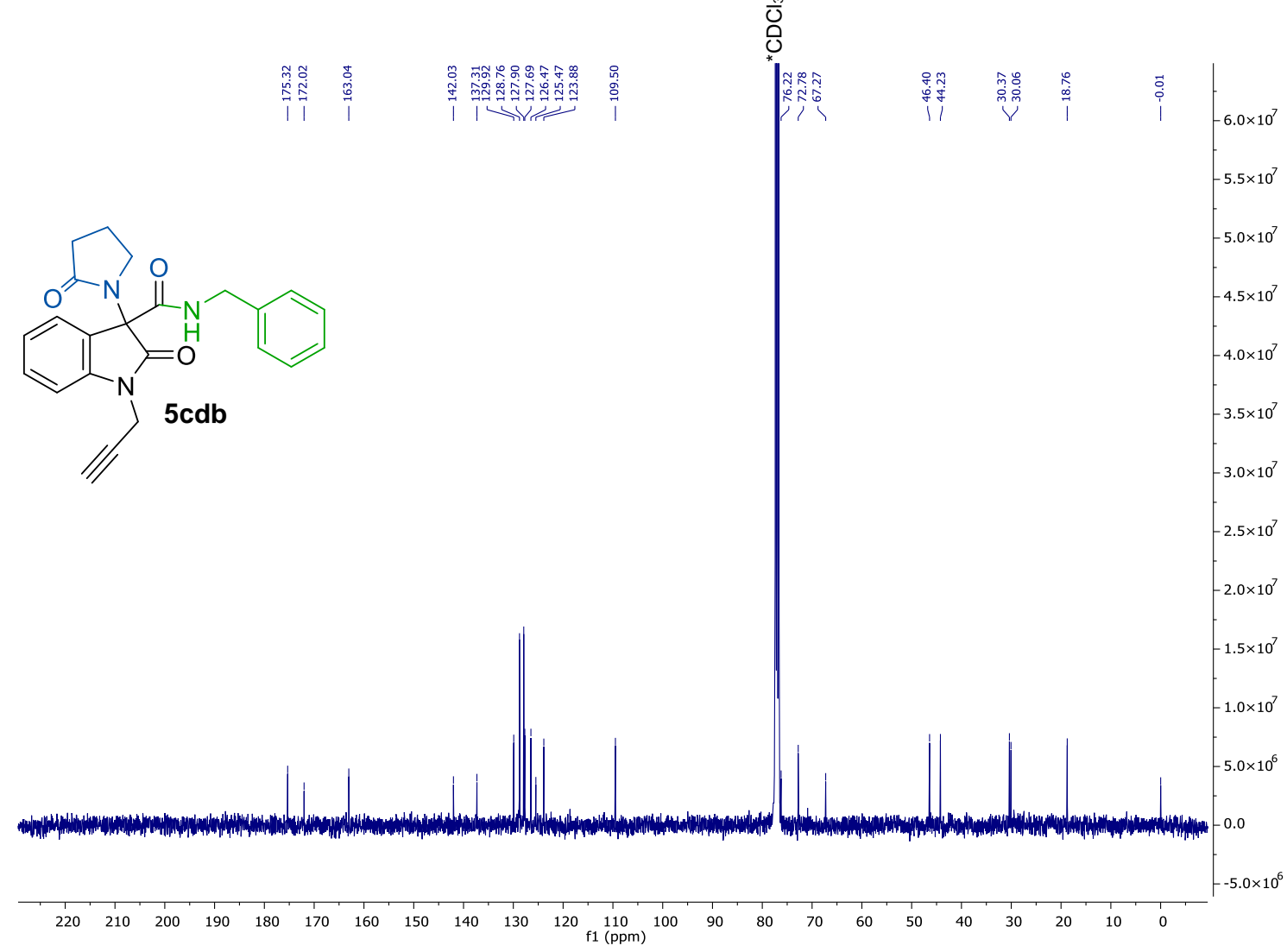




\section{High-Resolution Mass Spectra}

3.1. High-Resolution Mass Spectra of $\beta$-lactam-oxindole hybrids

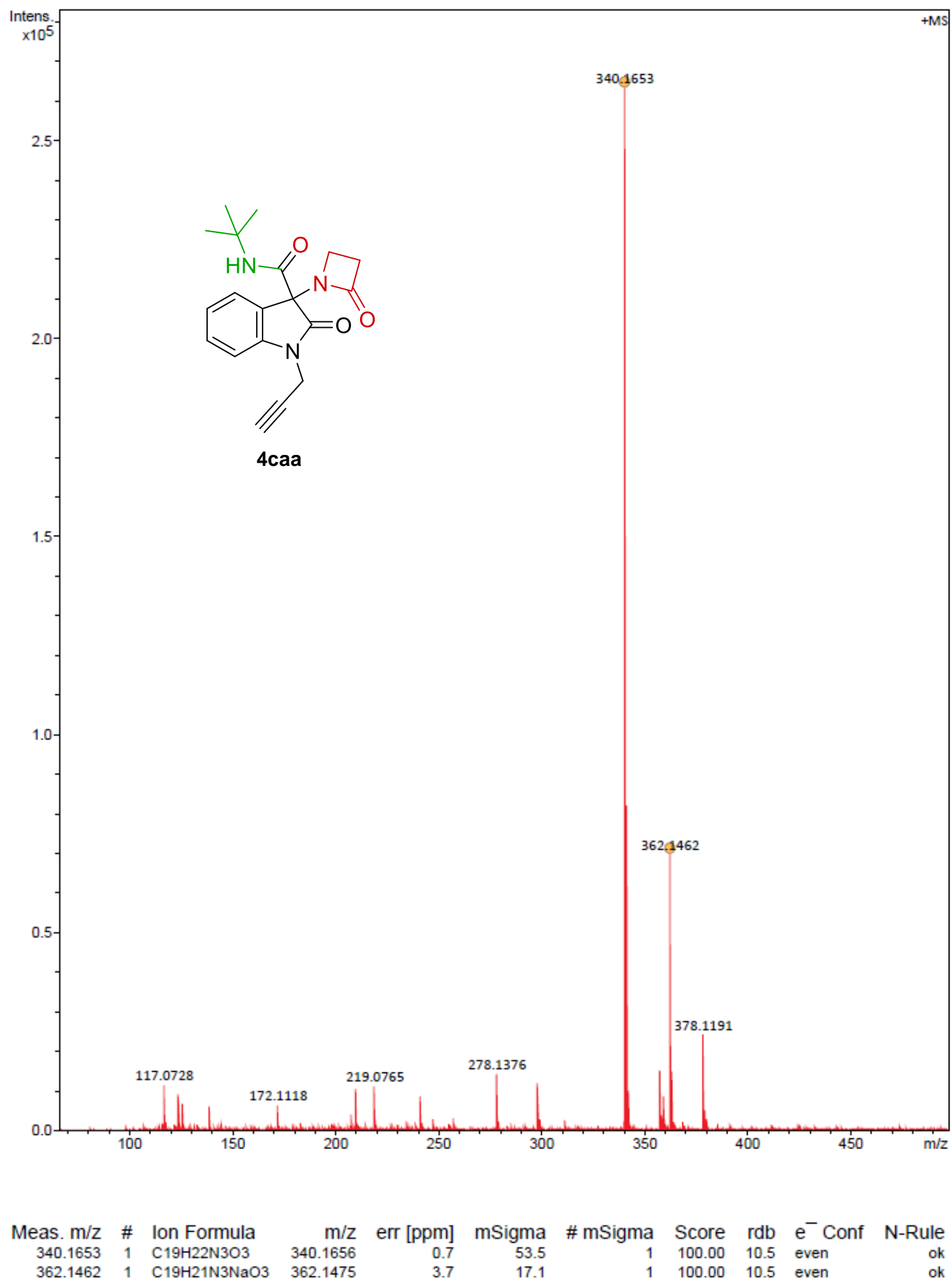


210203_055 \#15 RT: 0.14 AV: 1 NL: $2.40 E+009$

T: FTMS + p ESI Full ms [100.0000-1500.0000]
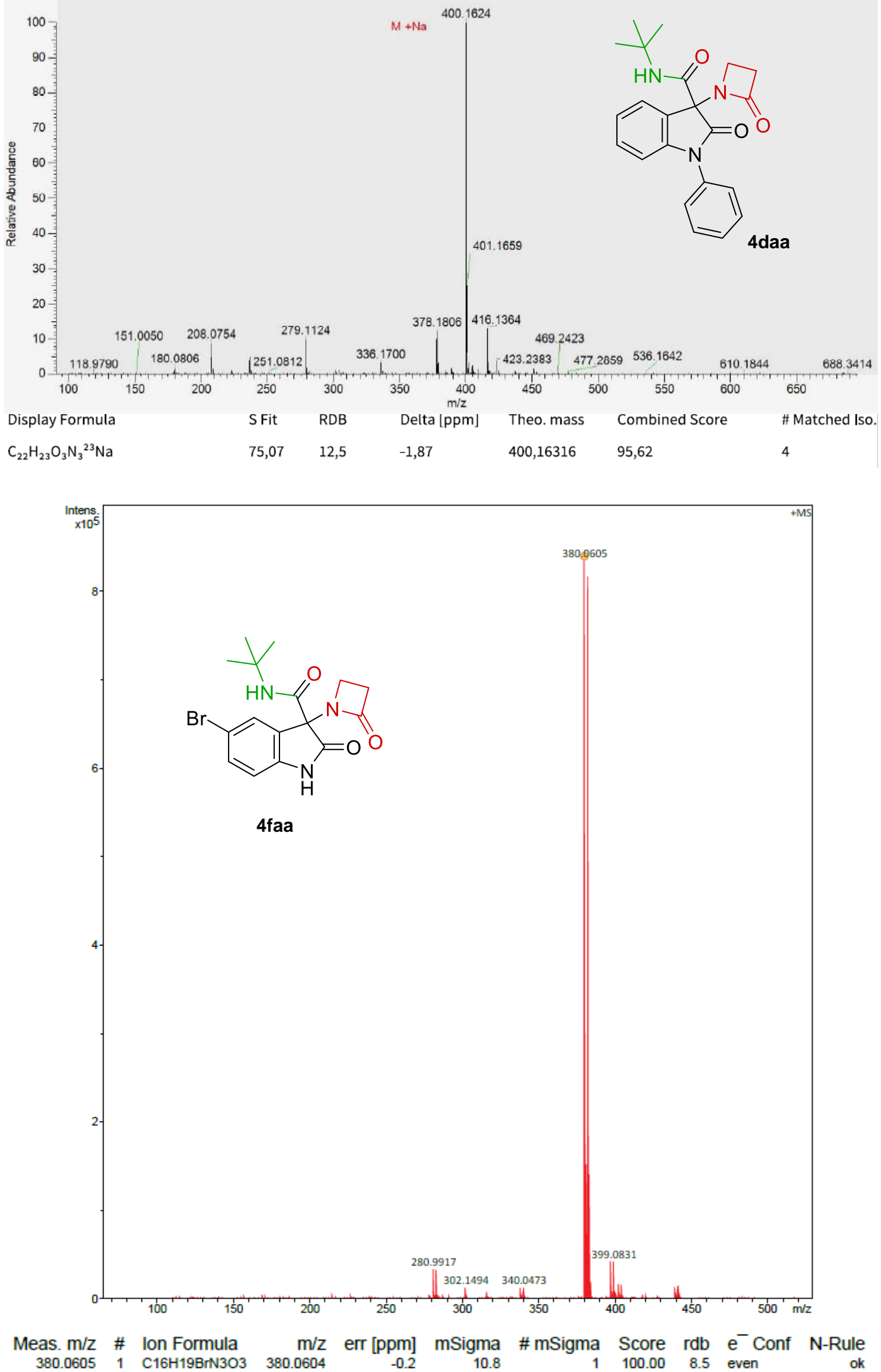

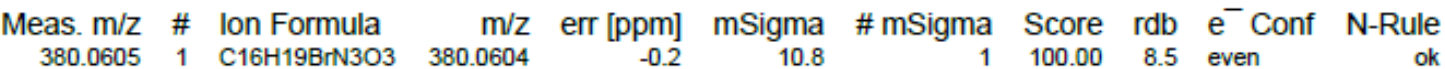




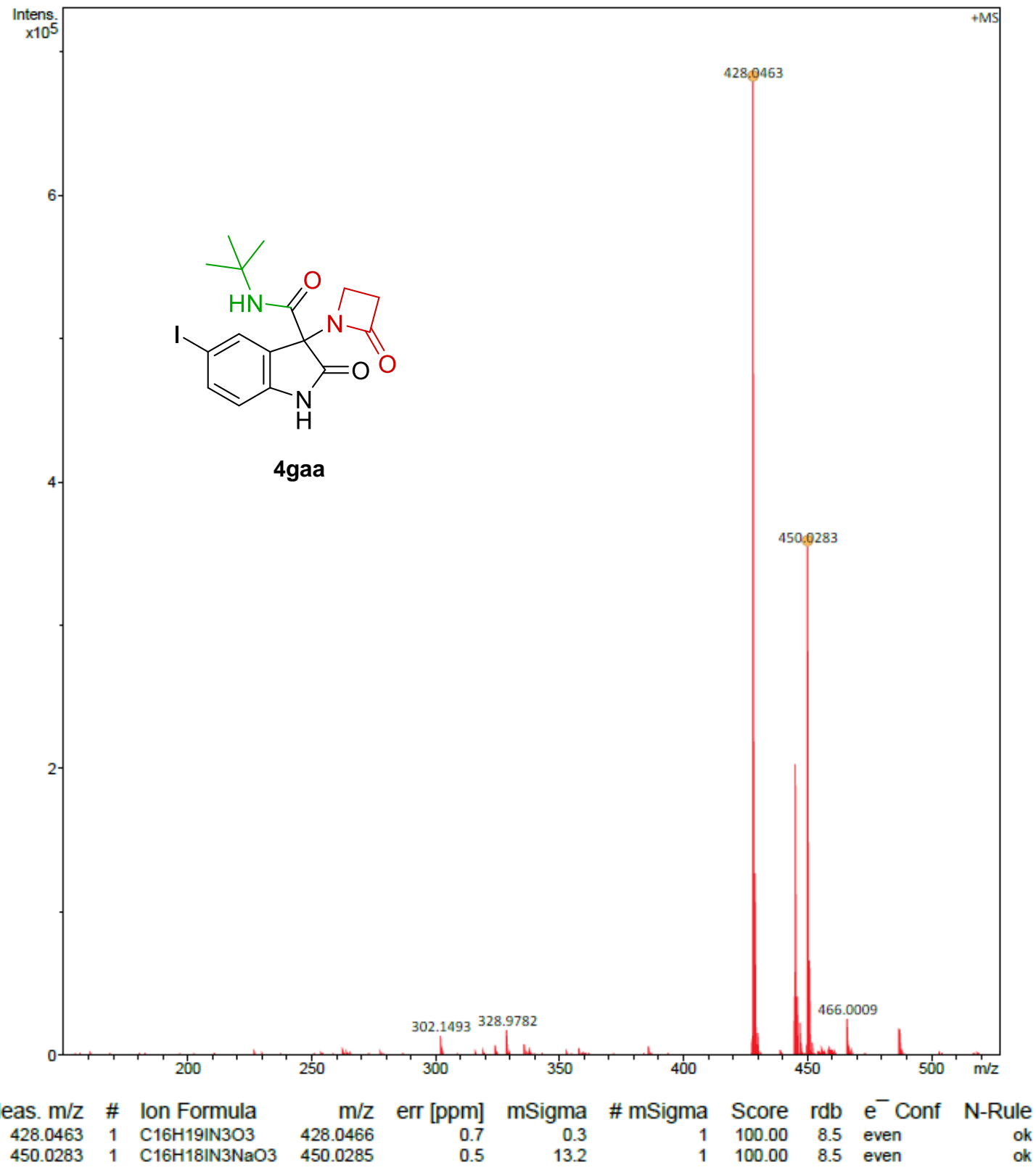




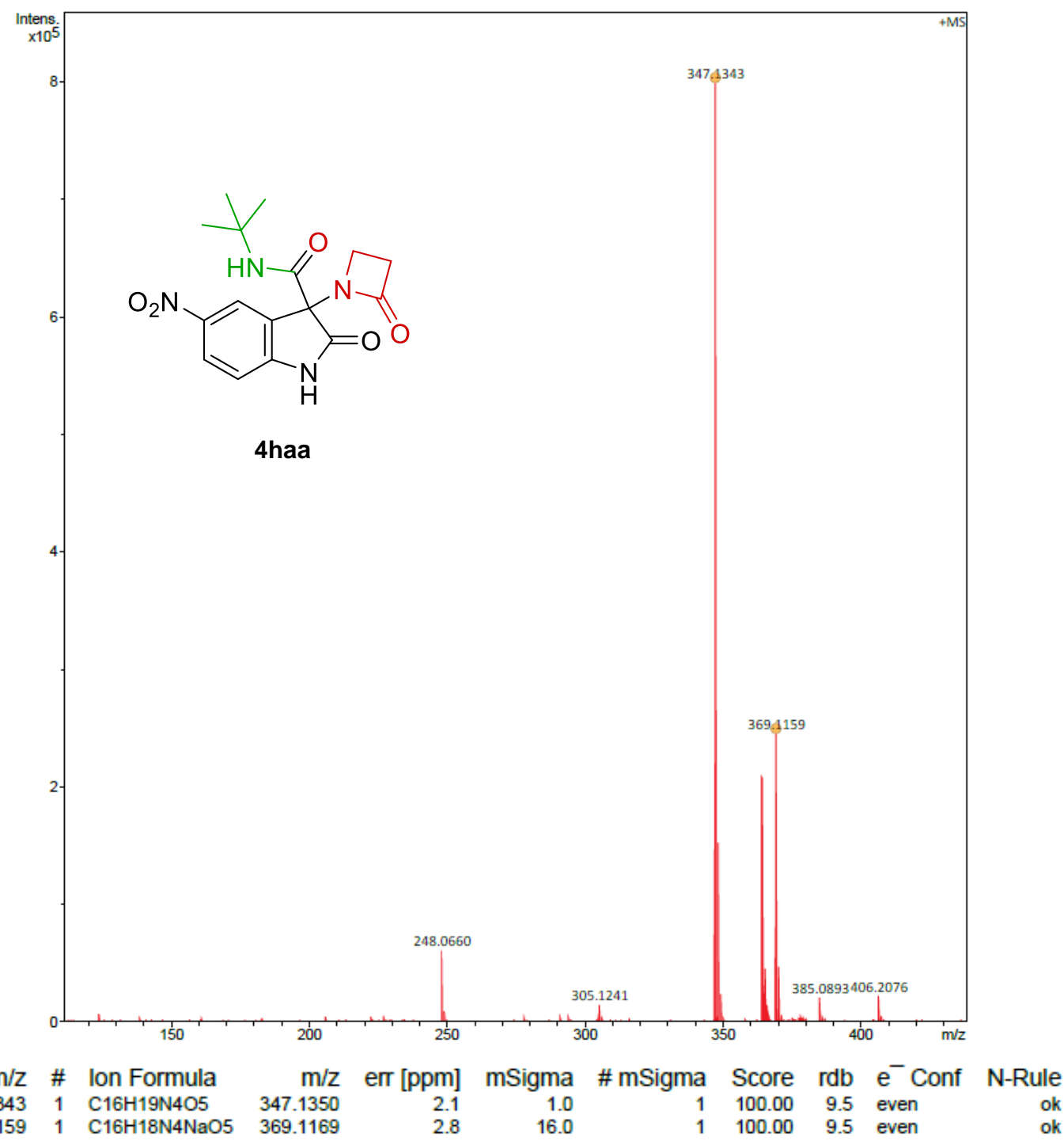

210203_056 \#15 RT: 0.14 AV: 1 NL: 3.12E+009

T: FTMS + p ESI Full ms [100.0000-1500.0000]

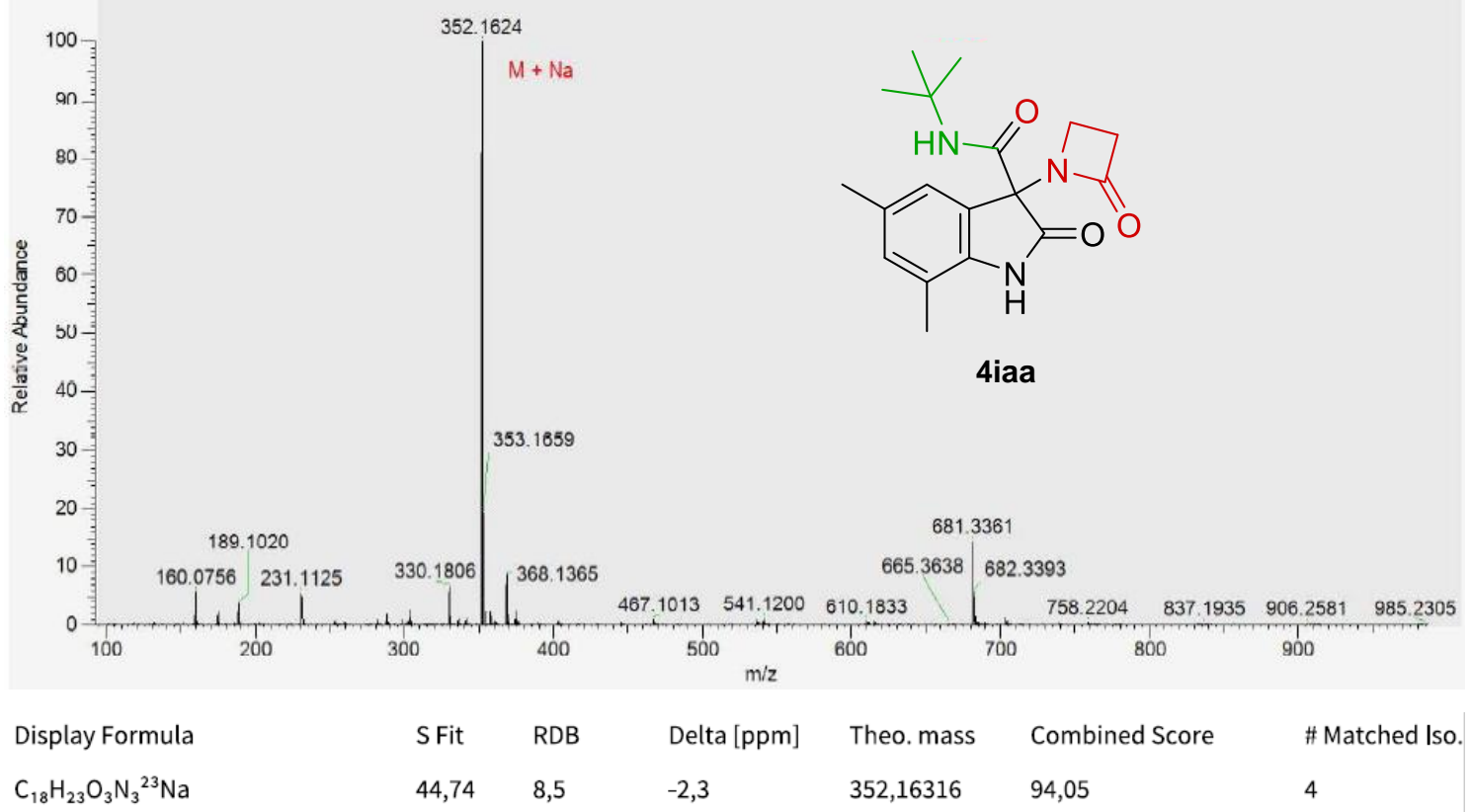


210203_057 \#15 RT: 0.14 AV: 1 NL: $2.30 E+009$

T: FTMS + p ESI Full ms [100.0000-1500.0000]

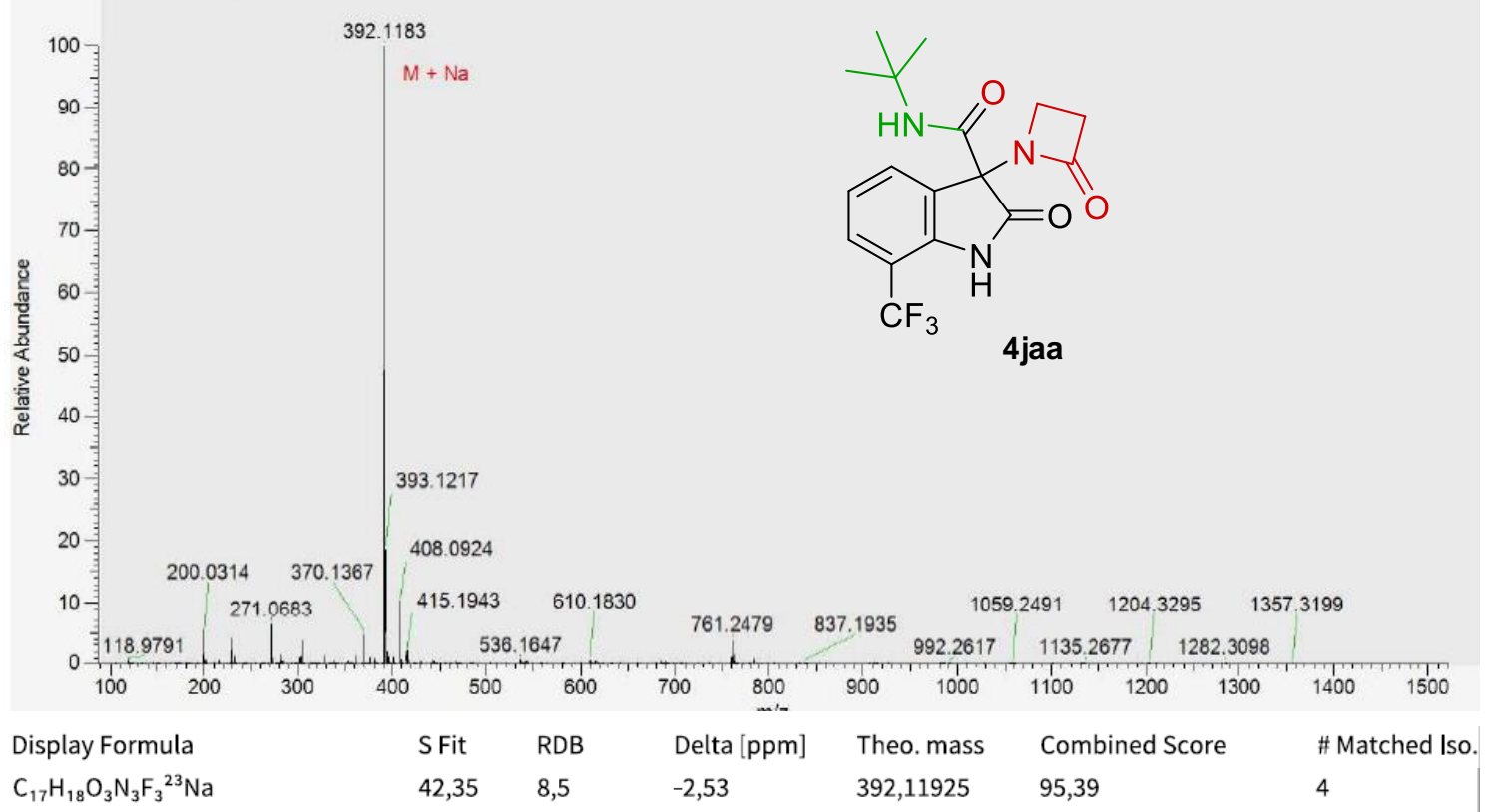

210203_058 \#15 RT: 0.14 AV: 1 NL: $2.32 E+009$

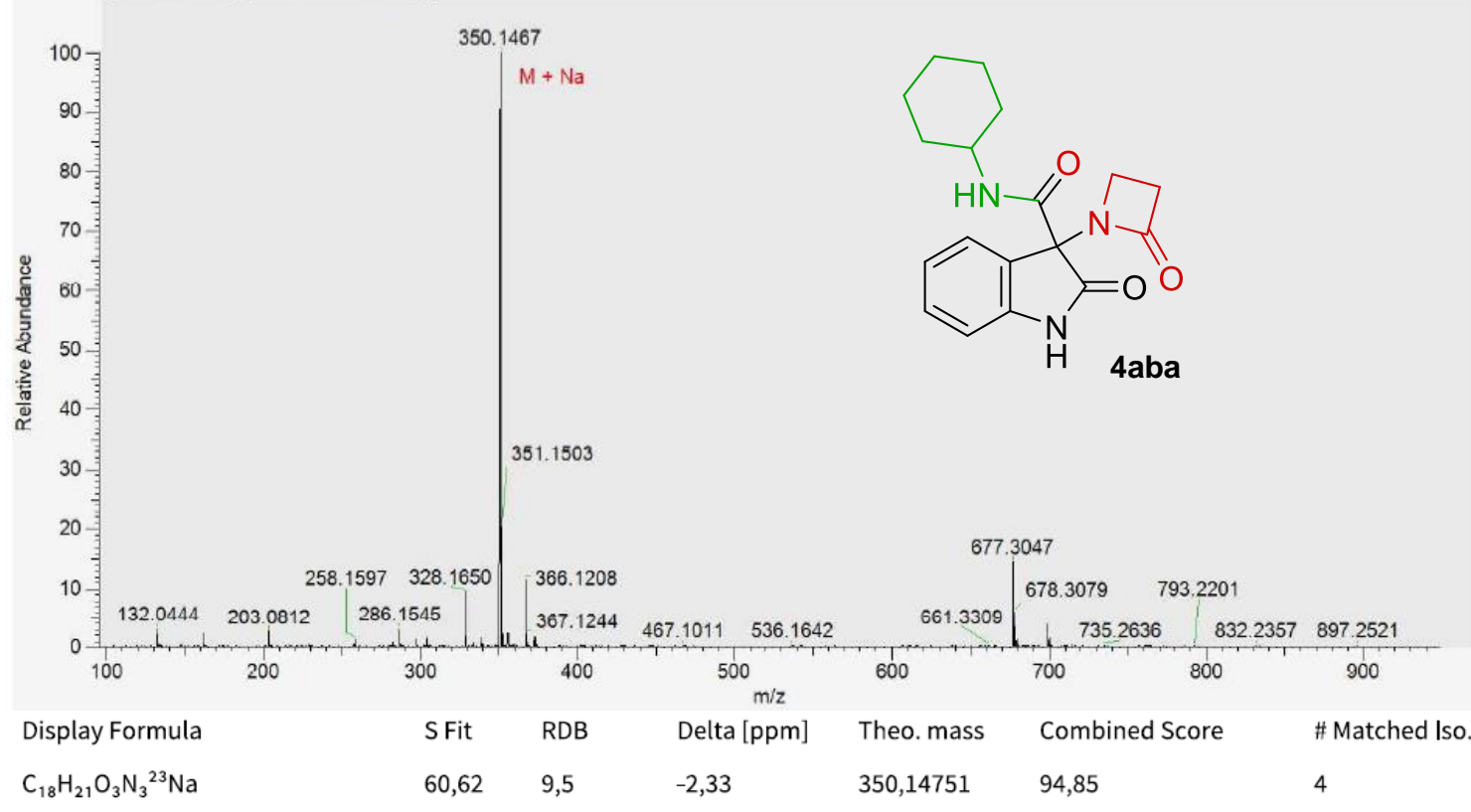


210203_059 \#15 RT: 0.14 AV: 1 NL: $1.85 E+009$

T: FTMS + p ESI Full ms [100,0000-1500,0000]

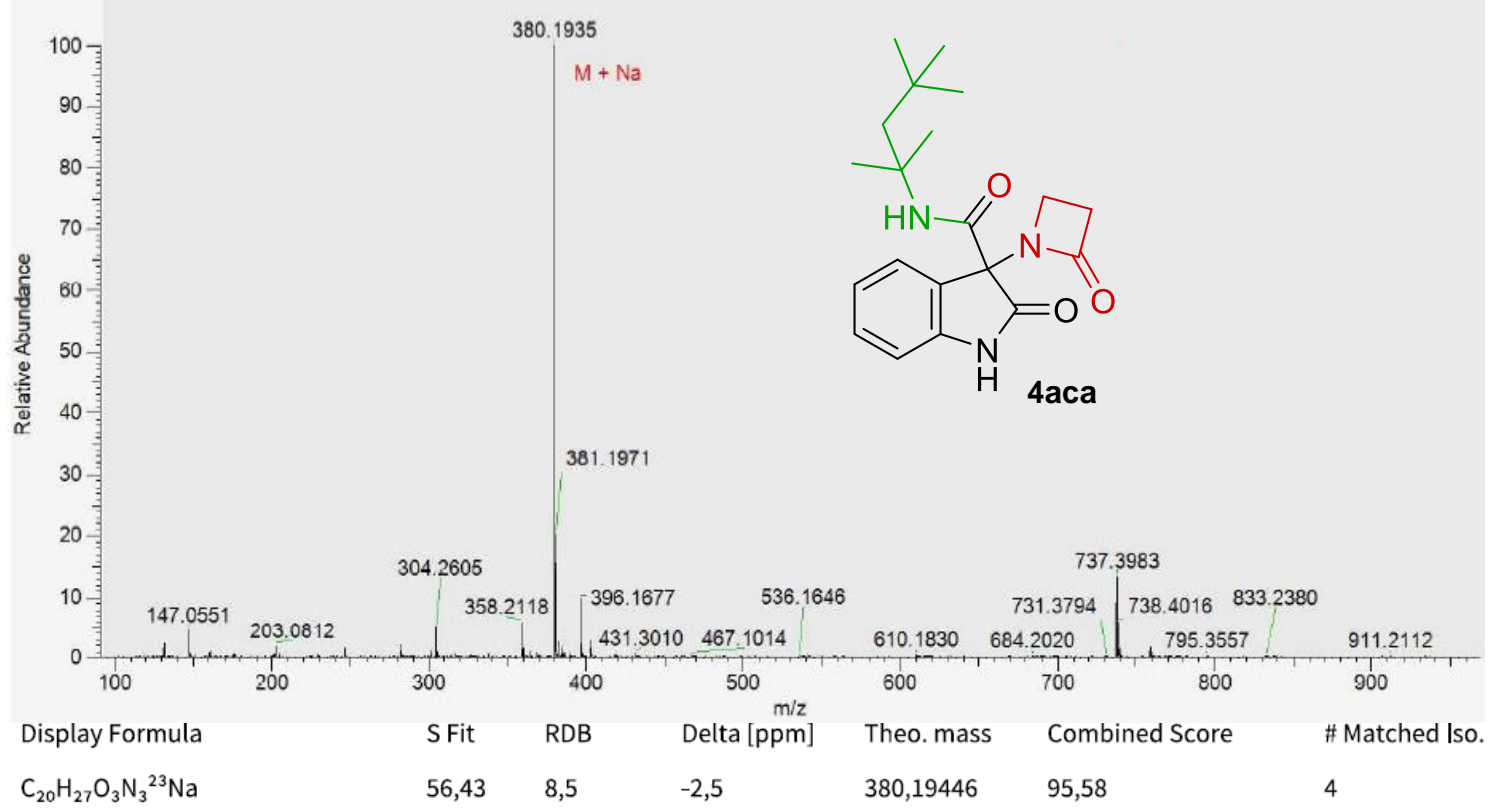

210203_062 \#15 RT: 0.14 AV: 1 NL: $1.28 E+009$

T. FTMS + PESI Full ms $100.0000-1500.0000$

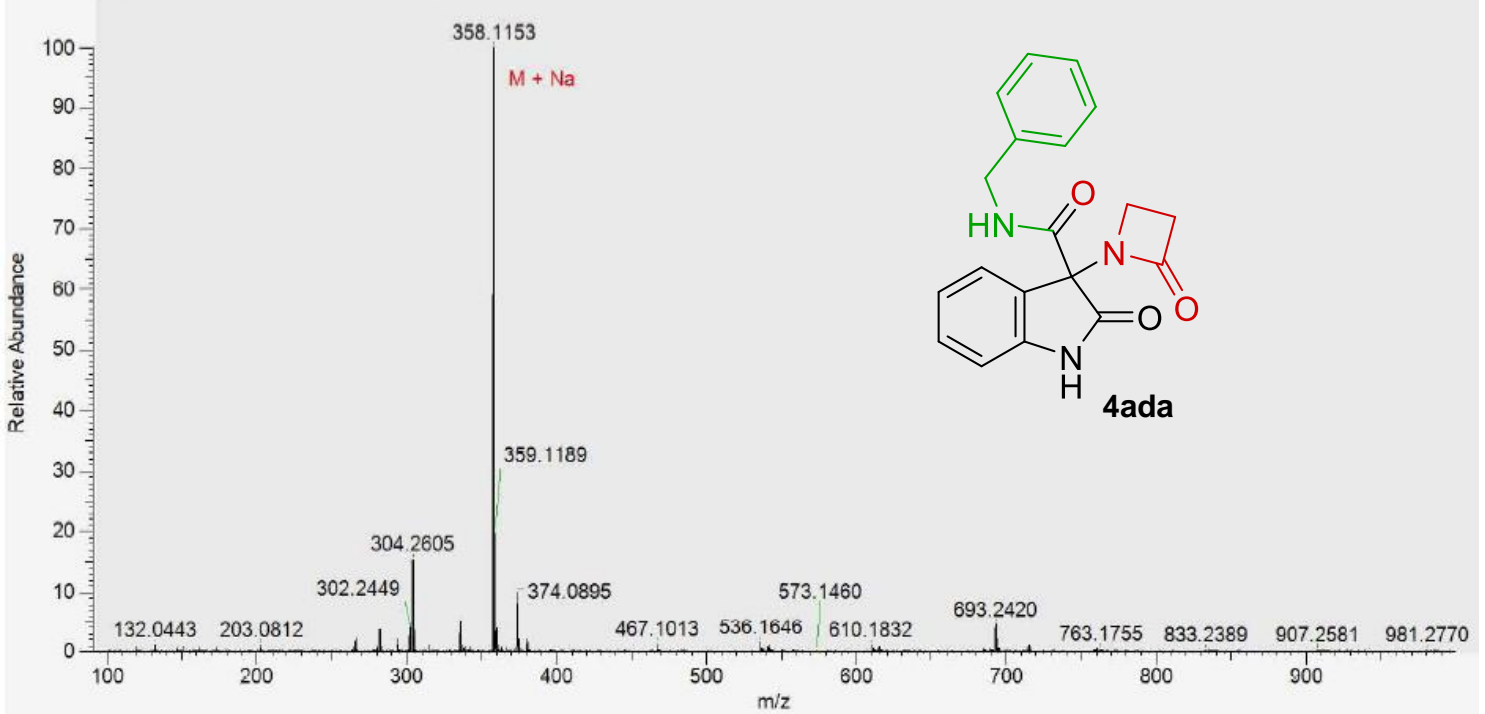

Display Formula

$\mathrm{C}_{19} \mathrm{H}_{17} \mathrm{O}_{3} \mathrm{~N}_{3}{ }^{23} \mathrm{Na}$
S Fit RDB

$44,23 \quad 12,5$
Delta [ppm] Theo. mass

$-2,48$

358,11621
Combined Score

92,45
\# Matched Iso.

4 
210203_063 \#15 RT: 0.14 AV: 1 NL: $2.01 E+009$

T: FTMS + p ESI Full ms [100.0000-1500.0000]

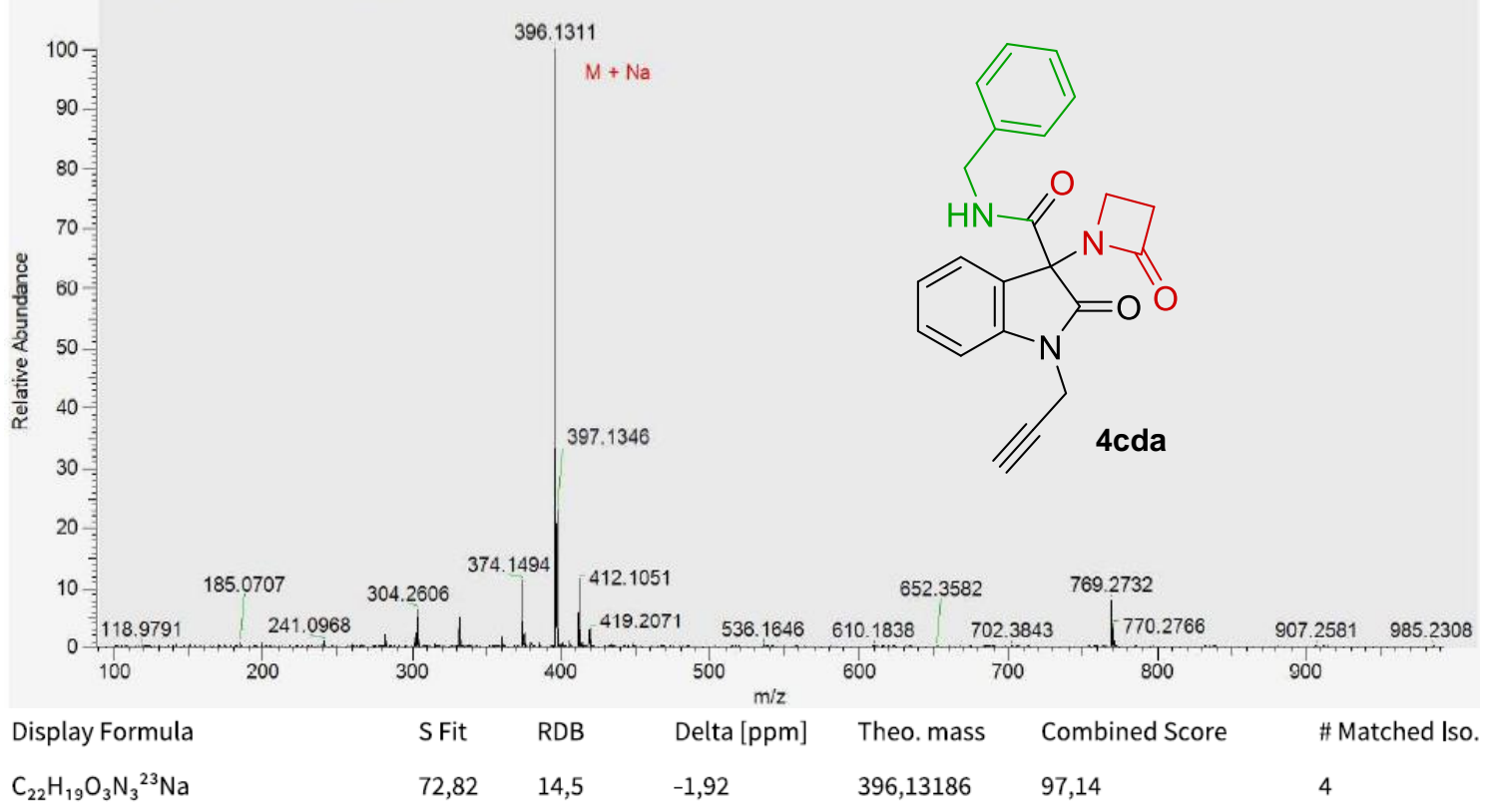

210203_061 \#15 RT: 0.14 AV: 1 NL: $3.71 E+009$

T. FTMS + p ESI Full ms [100.0000-1500.0000]

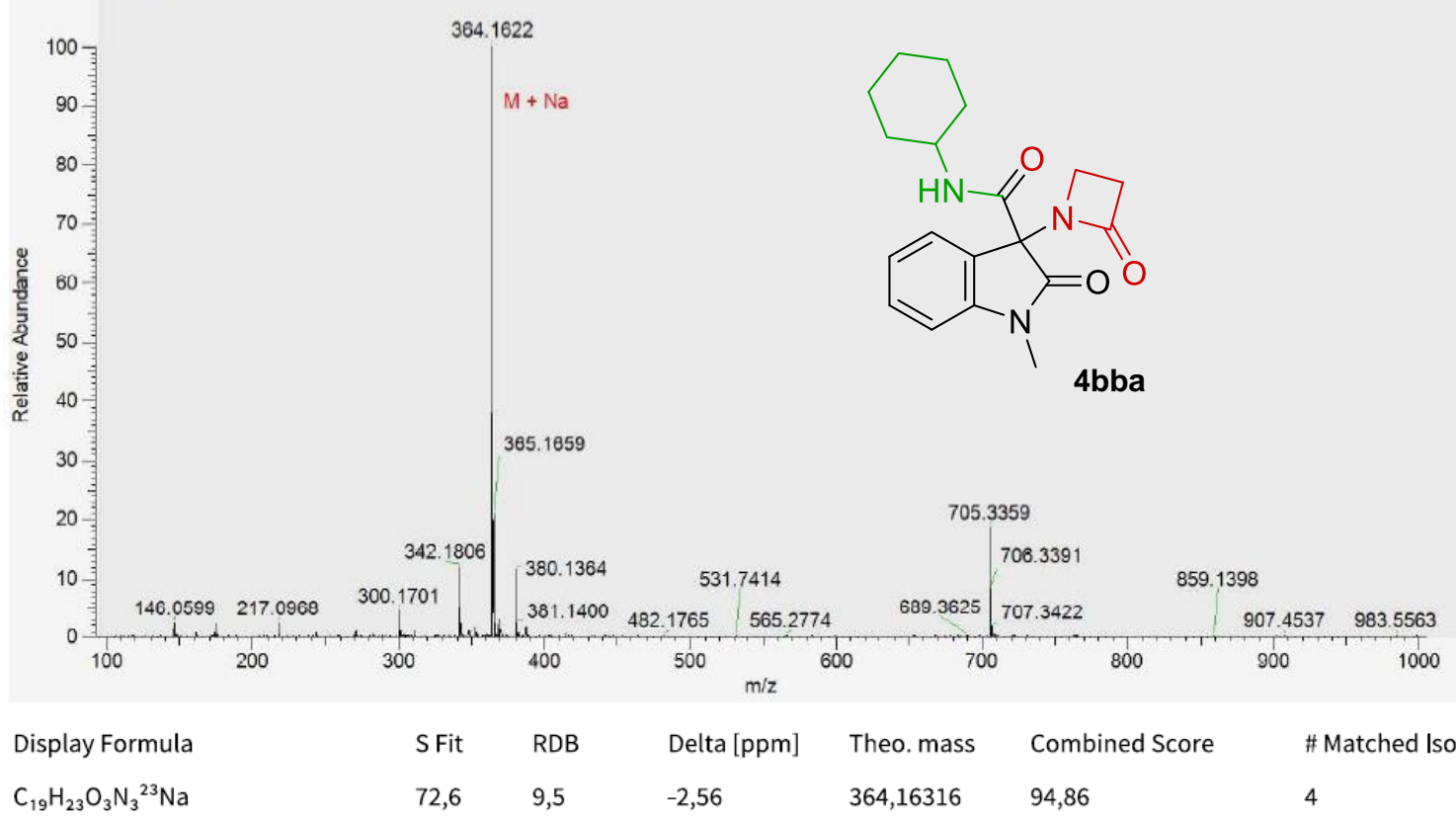


210203_060 \#15 RT: 0.14 AV: 1 NL: $2.68 E+009$

T: FTMS + p ESI Full ms [100.0000-1500.0000]

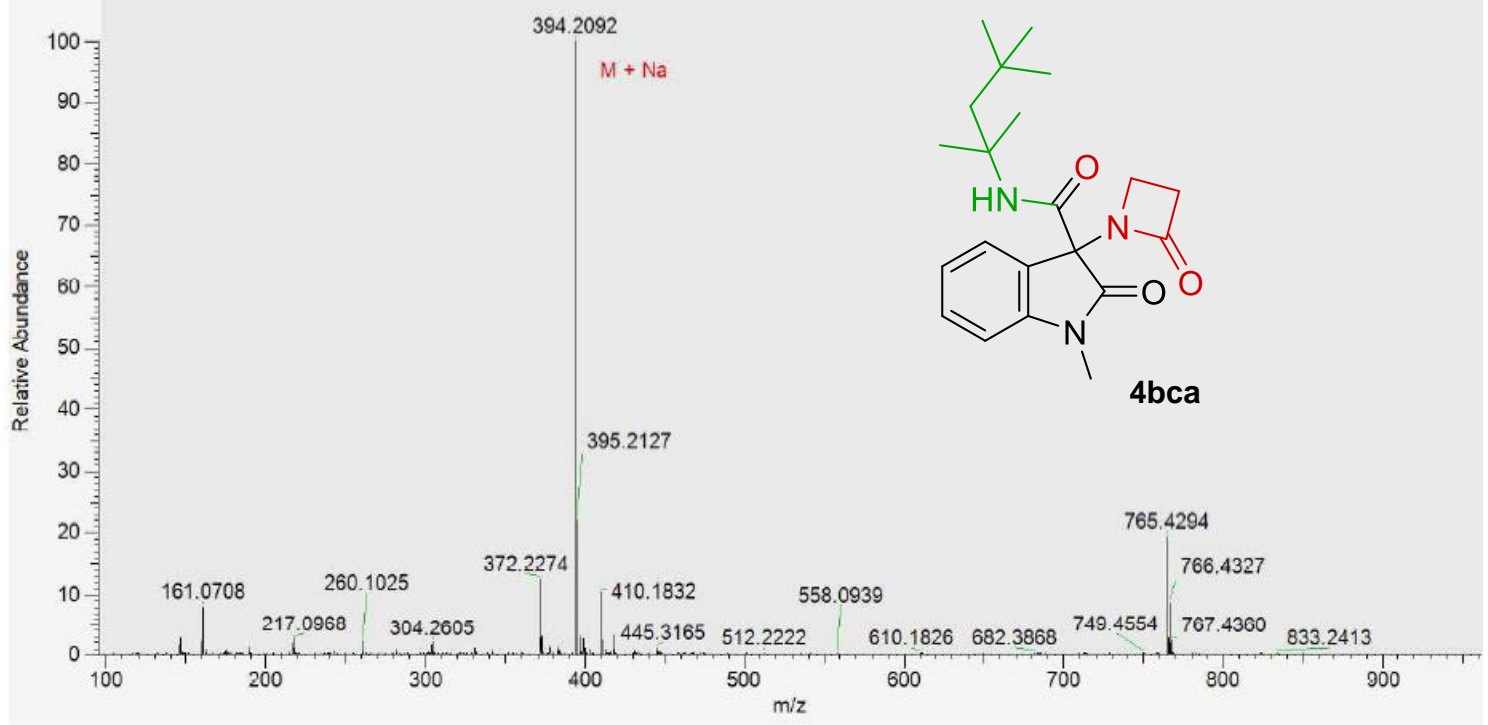

Display Formula

S Fit RDB

$\mathrm{C}_{21} \mathrm{H}_{29} \mathrm{O}_{3} \mathrm{~N}_{3}{ }^{23} \mathrm{Na}$

$74,32 \quad 8,5$

Delta [ppm]

Theo. mass

Combined Score

\# Matched Iso.

$-2,24$

394,21011

95,34

4 
3.2. High-Resolution Mass Spectra of $y$-lactam-oxindole hybrids

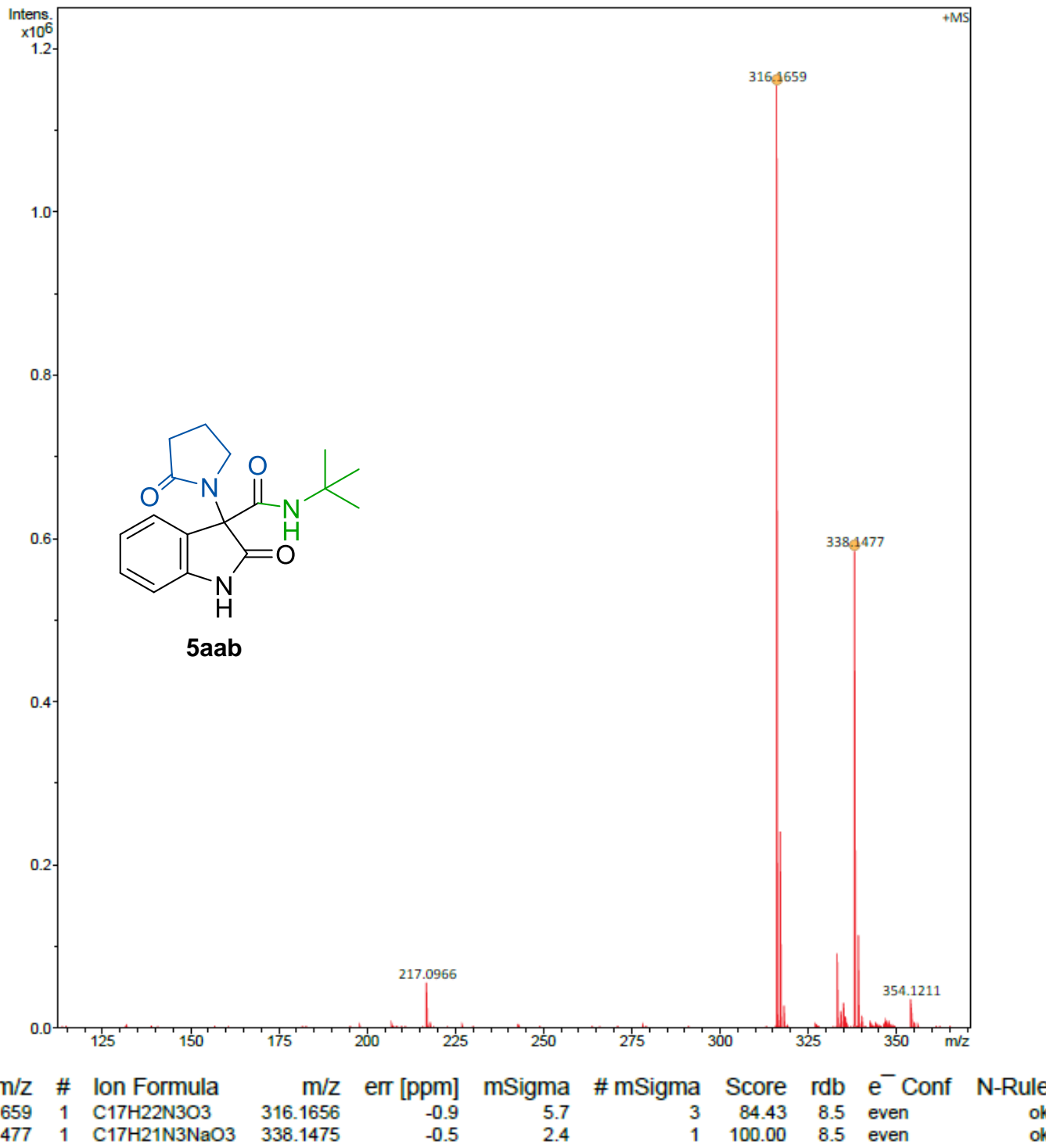

\begin{tabular}{|c|c|c|c|c|c|c|c|c|c|c|}
\hline Meas $m / z$ & \# & Ion Formula & $\mathrm{m} / \mathrm{z}$ & err [ppm] & mSigma & \# mSigma & Score & $\mathrm{rdb}$ & $\mathrm{e}^{-}$Conf & tule \\
\hline 3 & 1 & $\mathrm{C} 17 \mathrm{H} 22 \mathrm{~N} 3 \mathrm{O} 3$ & 316.1656 & -0.9 & 5.7 & 3 & 43 & & even & \\
\hline 38.147 & 1 & $\mathrm{C} 17 \mathrm{H} 21 \mathrm{~N} 3 \mathrm{NaO} 3$ & 338.1475 & -0.5 & 2.4 & 1 & 100.00 & 8.5 & even & \\
\hline
\end{tabular}

210203_064 \#15 RT: 0.14 AV: 1 NL: $2.40 E+009$

T: FTMS + p ESI Full ms [100.0000-1500.0000]

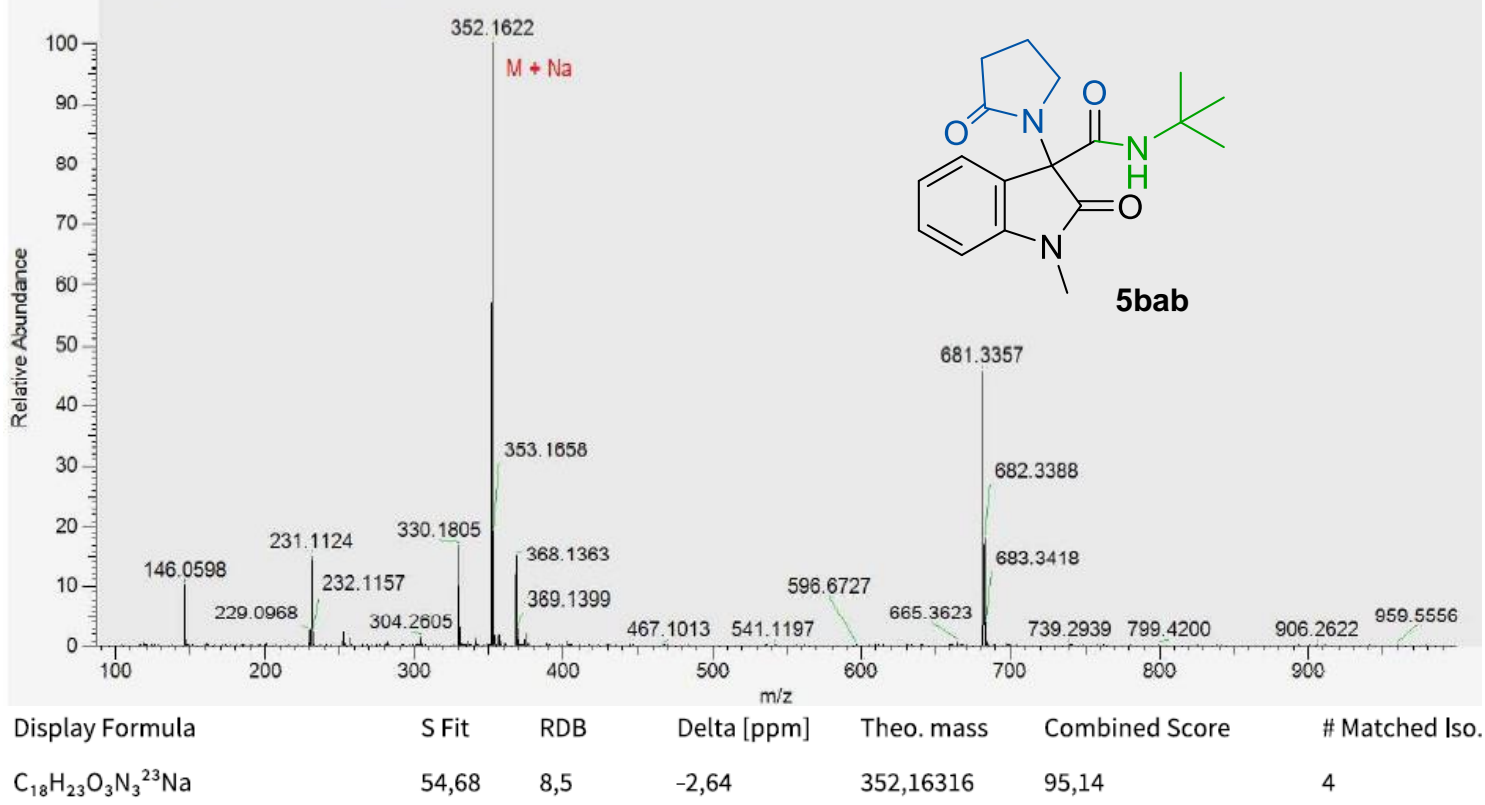


210203068 \#15 RT: 0.14 AV: 1 NL: $1.65 E+009$

T: FTMS + p ESI Full ms [100.0000-1500.0000]

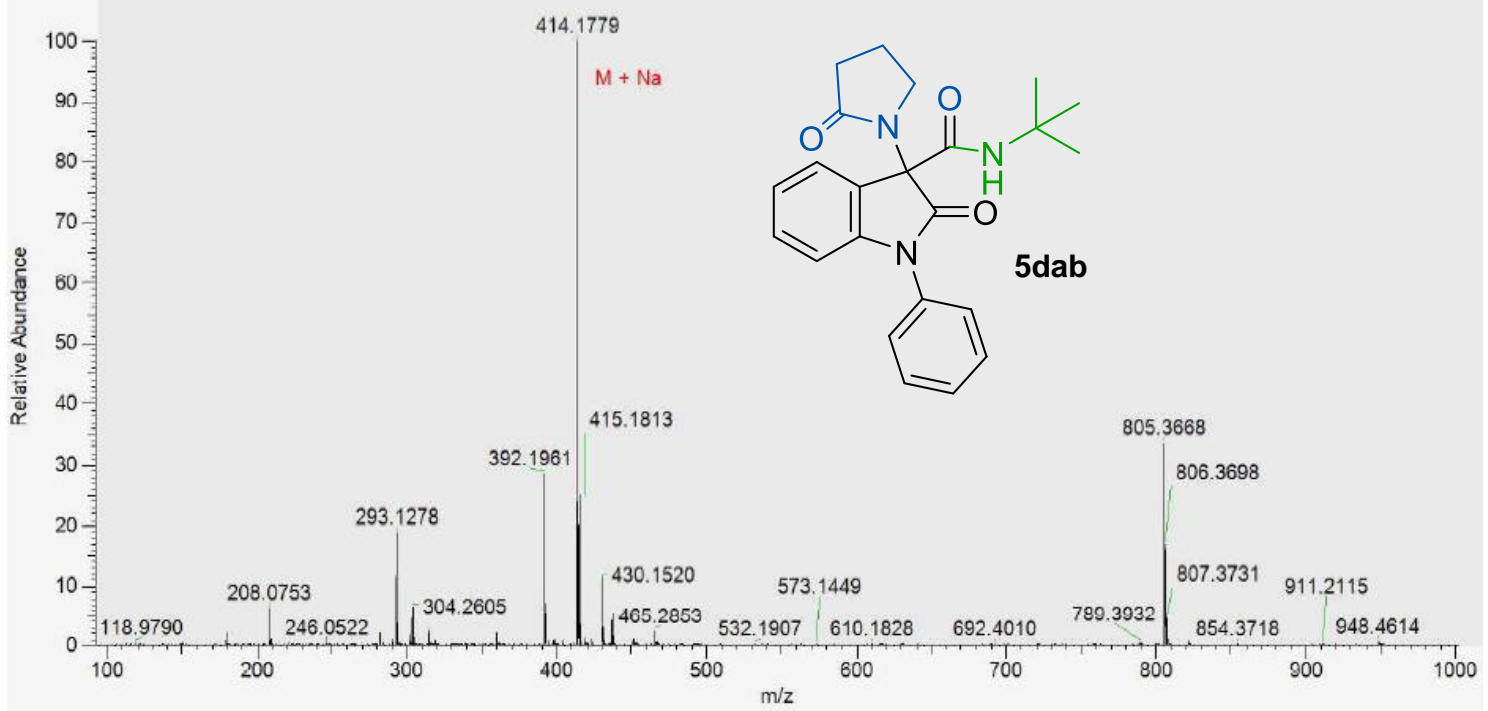

$\begin{array}{lllllll}\text { Display Formula } & \text { S Fit } & \text { RDB } & \text { Delta [ppm] } & \text { Theo. mass } & \text { Combined Score } \\ \mathrm{C}_{23} \mathrm{H}_{25} \mathrm{O}_{3} \mathrm{~N}_{3}{ }^{23} \mathrm{Na} & 58,16 & 12,5 & -2,24 & 414,17881 & 96,15\end{array}$

210203_069 \#15 RT: 0.14 AV: 1 NL: 2.16E+009

T: FTMS + p ESI Full ms [100.0000-1500.0000]

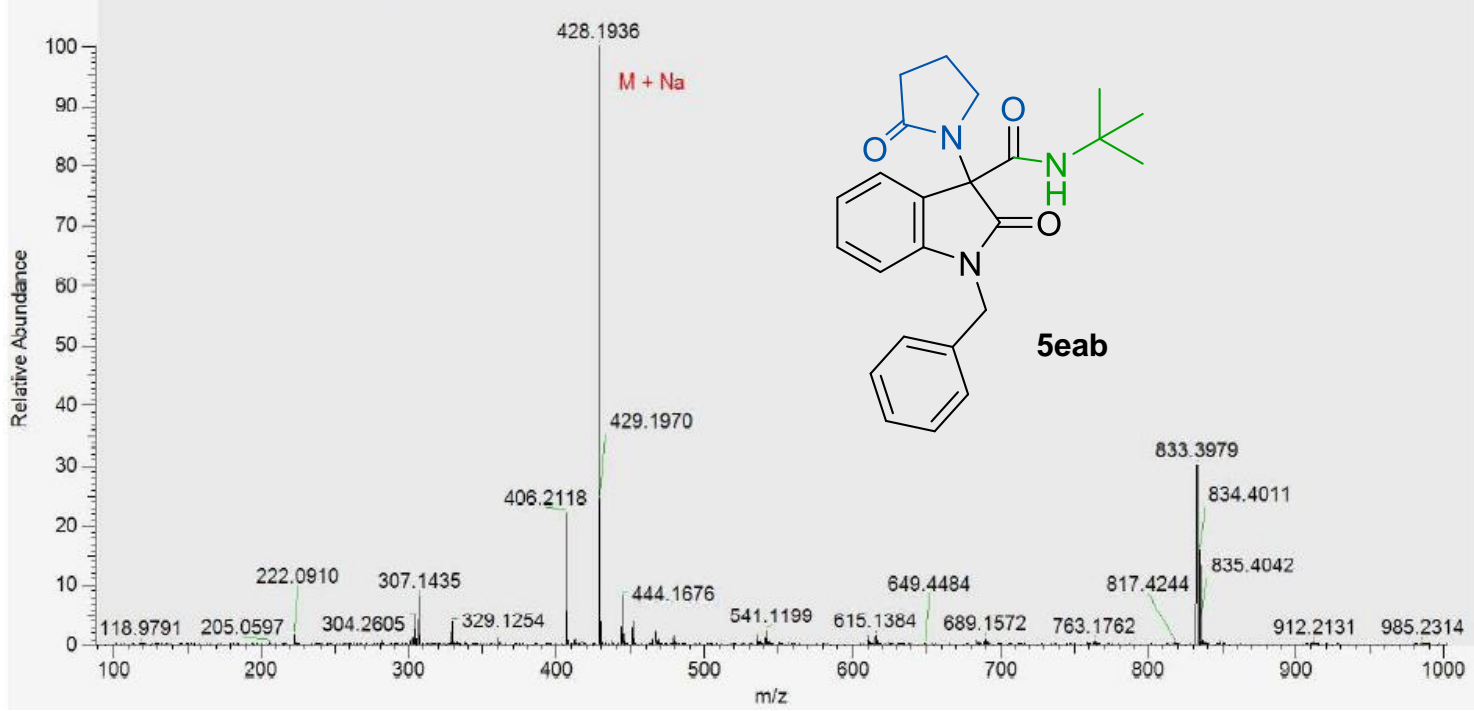

Display Formula

S Fit $\quad$ RD

Delta [ppm] Theo. mass

Combined Score

\# Matched Iso.

$\mathrm{C}_{24} \mathrm{H}_{27} \mathrm{O}_{3} \mathrm{~N}_{3}{ }^{23} \mathrm{Na}$

76,7

12,5

$-1,94$

428,19446

97,89

4 


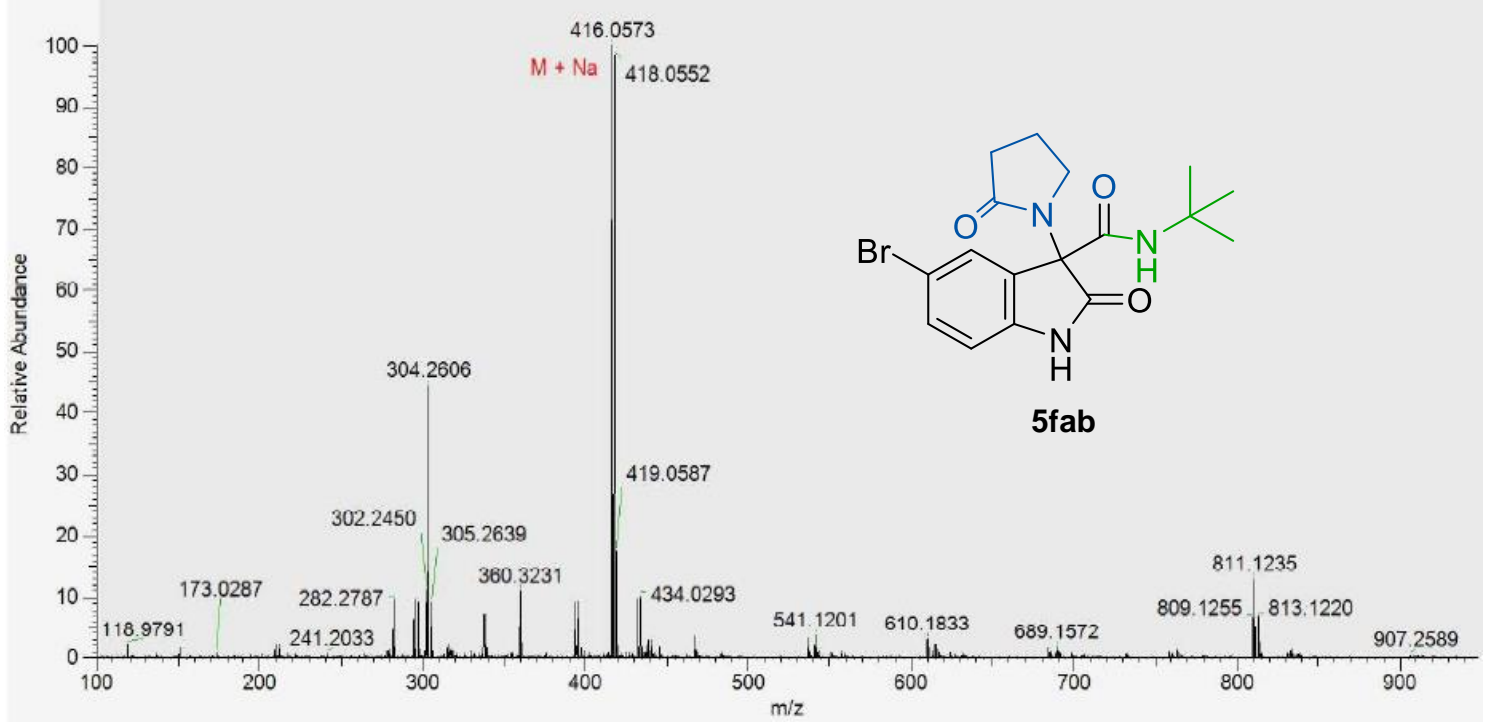

$\begin{array}{lllllll}\text { Display Formula } & \text { S Fit } & \text { RDB } & \text { Delta [ppm] } & \text { Theo. mass } & \text { Combined Score } & \text { \# Matched Iso. } \\ \mathrm{C}_{17} \mathrm{H}_{20} \mathrm{O}_{3} \mathrm{~N}_{3}{ }^{79} \mathrm{Br}^{23} \mathrm{Na} & 53,36 & 8,5 & -1,64 & 416,05803 & 96,76 & 6\end{array}$
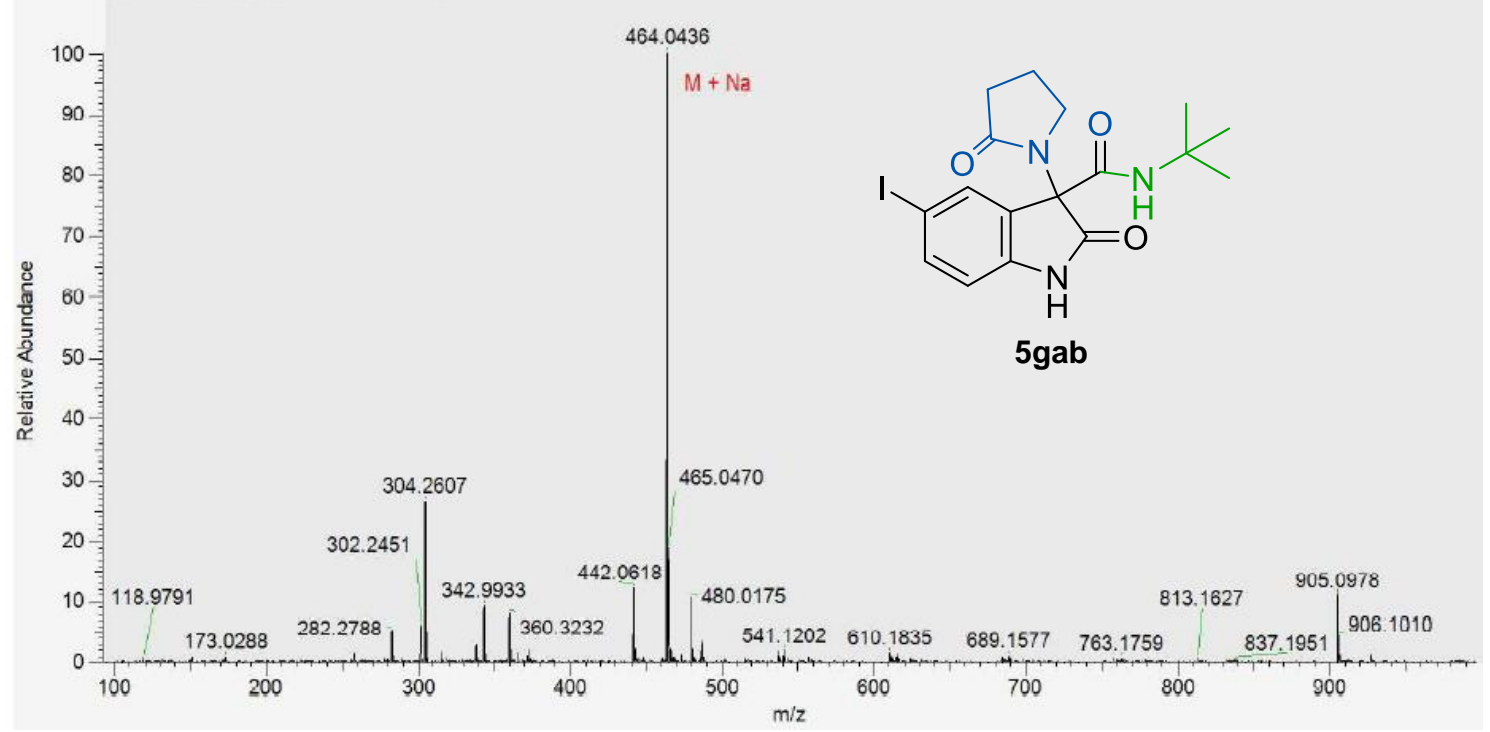

Display Formula

$\begin{array}{ll}\text { S Fit } & \text { RDB } \\ 74,46 & 8,5\end{array}$

Delta [ppm

Theo. mass

Combined Score

\# Matched Iso.

$\left.\mathrm{C}_{17} \mathrm{H}_{20} \mathrm{O}_{3} \mathrm{~N}_{3}{ }^{127}\right|^{23} \mathrm{Na}$

$74,46 \quad 8,5$

$-1,24$

$464,04416 \quad 95,36$

4 
210203_074 \#15 RT: 0.14 AV: 1 NL: $4.02 E+008$

T: FTMS $+p$ ESI Full ms [100.0000-1500.0000]

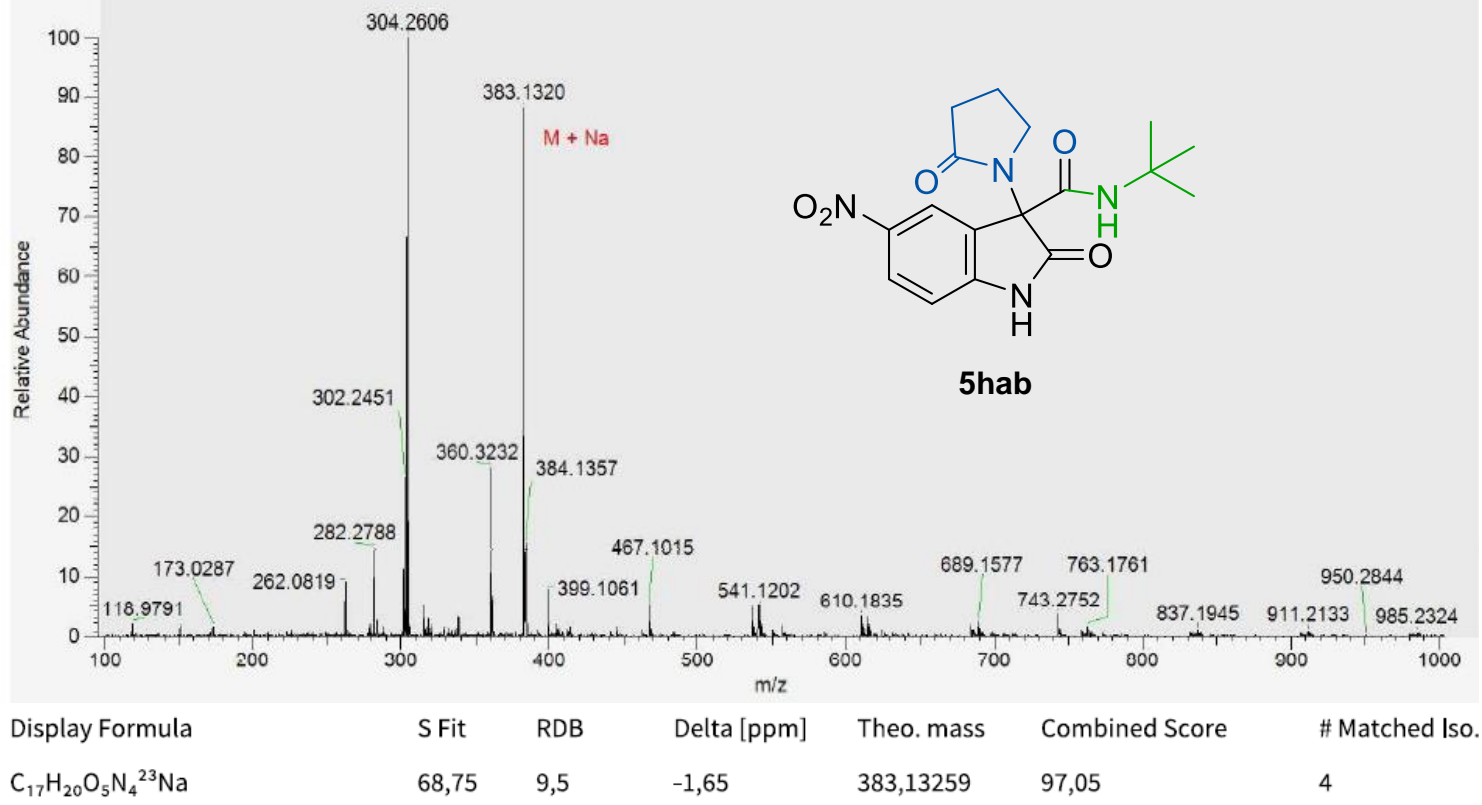

210203_070 \#15 RT: 0.14 AV: 1 NL: $1.88 E+009$ T: FTMS + p ESI Full ms [100.0000-1500.0000]

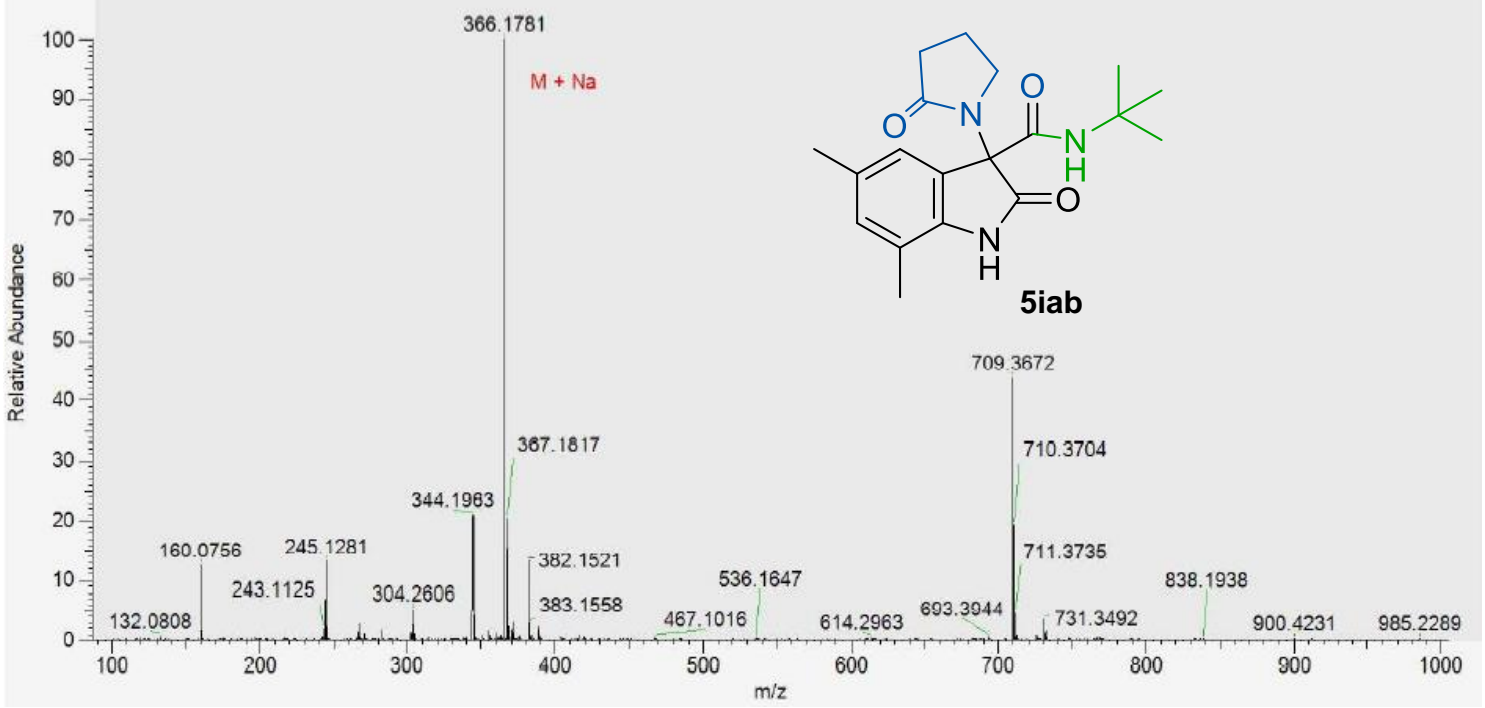

Display Formula

S Fit RDB

Theo. mass

Combined Score

\# Matched Iso.

$\mathrm{C}_{19} \mathrm{H}_{25} \mathrm{O}_{3} \mathrm{~N}_{3}{ }^{23} \mathrm{Na}$

$77,24 \quad 8,5$

$-1,95$

366,17881

95,02

4 
210203_072 \#15 RT: 0.14 AV: 1 NL: $7.99 E+008$

T: FTMS + p ESI Full ms [100.0000-1500.0000]

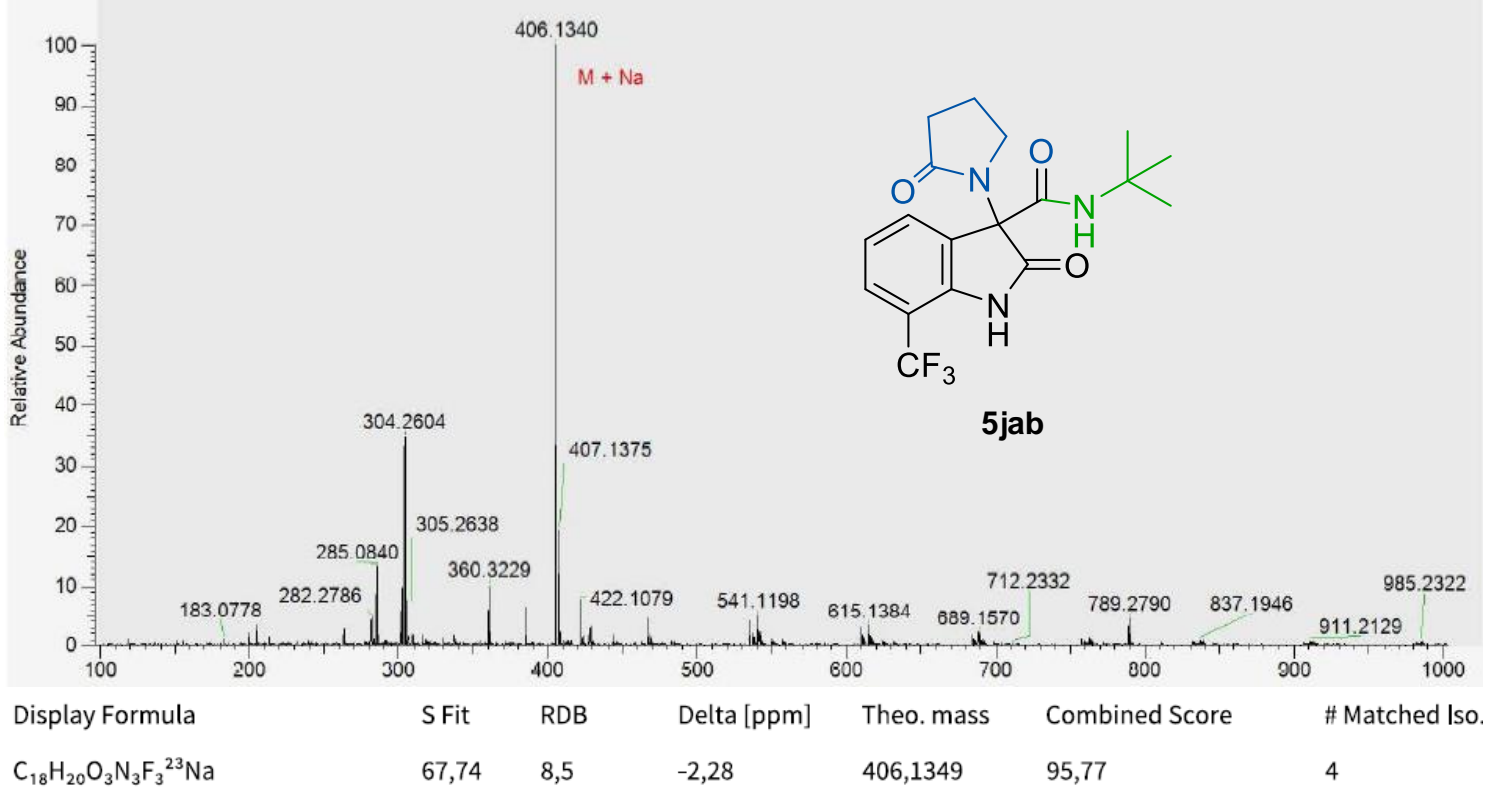

210203_065 \#15 RT: 0.14 AV: 1 NL: $1.13 E+009$

T. FTMS + p ESI Full ms [100.0000-1500.0000]

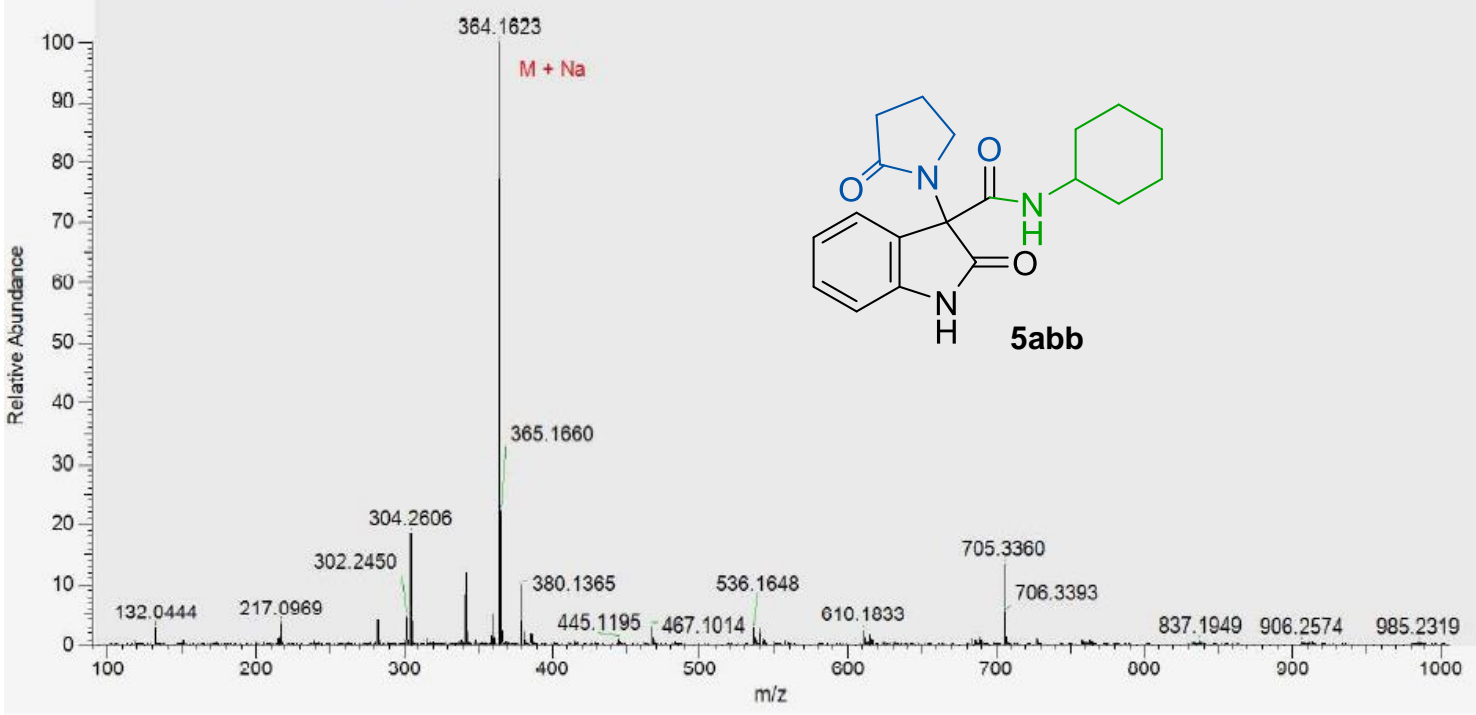

Display Formula

RDB

Delta $[p p m]$

Theo. mass

Combined Score

\# Matched Iso.

$\mathrm{C}_{19} \mathrm{H}_{23} \mathrm{O}_{3} \mathrm{~N}_{3}{ }^{23} \mathrm{Na}$

$65,21 \quad 9,5 \quad-2,31$

$364,16316 \quad 97,47$ 
210203_066 \#15 RT: 0.14 AV: 1 NL: $1.75 E+009$

T: FTMS + p ESI Full ms [100.0000-1500.0000]

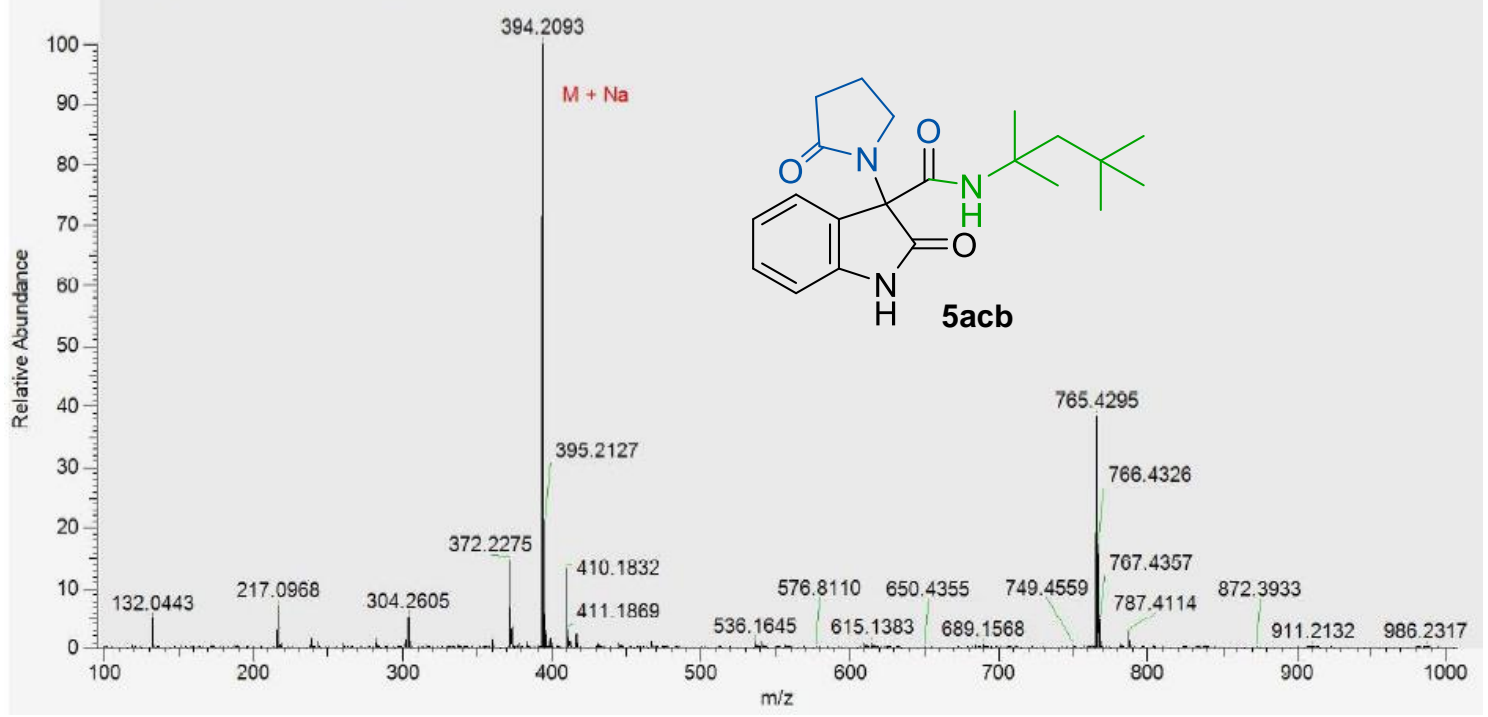

Display Formula

ombined Score

\# Matched Iso.

$\mathrm{C}_{21} \mathrm{H}_{29} \mathrm{O}_{3} \mathrm{~N}_{3}{ }^{23} \mathrm{Na}$

$58,16 \quad 8,5 \quad-2,09$

394,21011

95,91

4

210203_067 \#15 RT: 0.14 AV: 1 NL: $1.72 E+009$

T: FTMS + p ESI Full ms [100.0000-1500.0000]

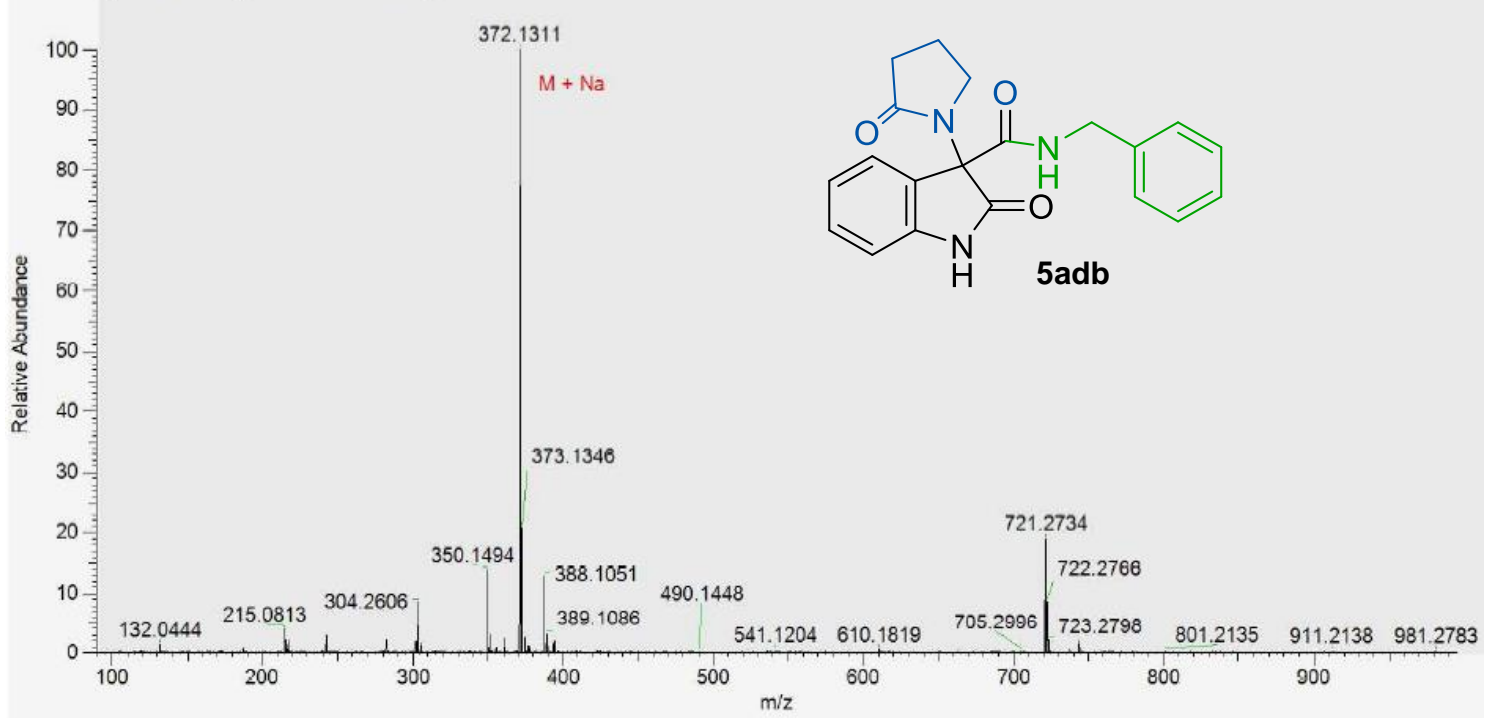

Display Formula

$\begin{array}{llllll}\text { S Fit } & \text { RDB } & \text { Delta }[\mathrm{ppm}] & \text { Theo. mass } & \text { Combined Score } & \text { \# Matched Iso. } \\ 74,57 & 12,5 & -2,04 & 372,13186 & 96,9 & 4\end{array}$


210203_073 \#15 RT: 0.14 AV: 1 NL: $1.49 E+009$

T: FTMS + p ESI Full ms [100.0000-1500.0000]

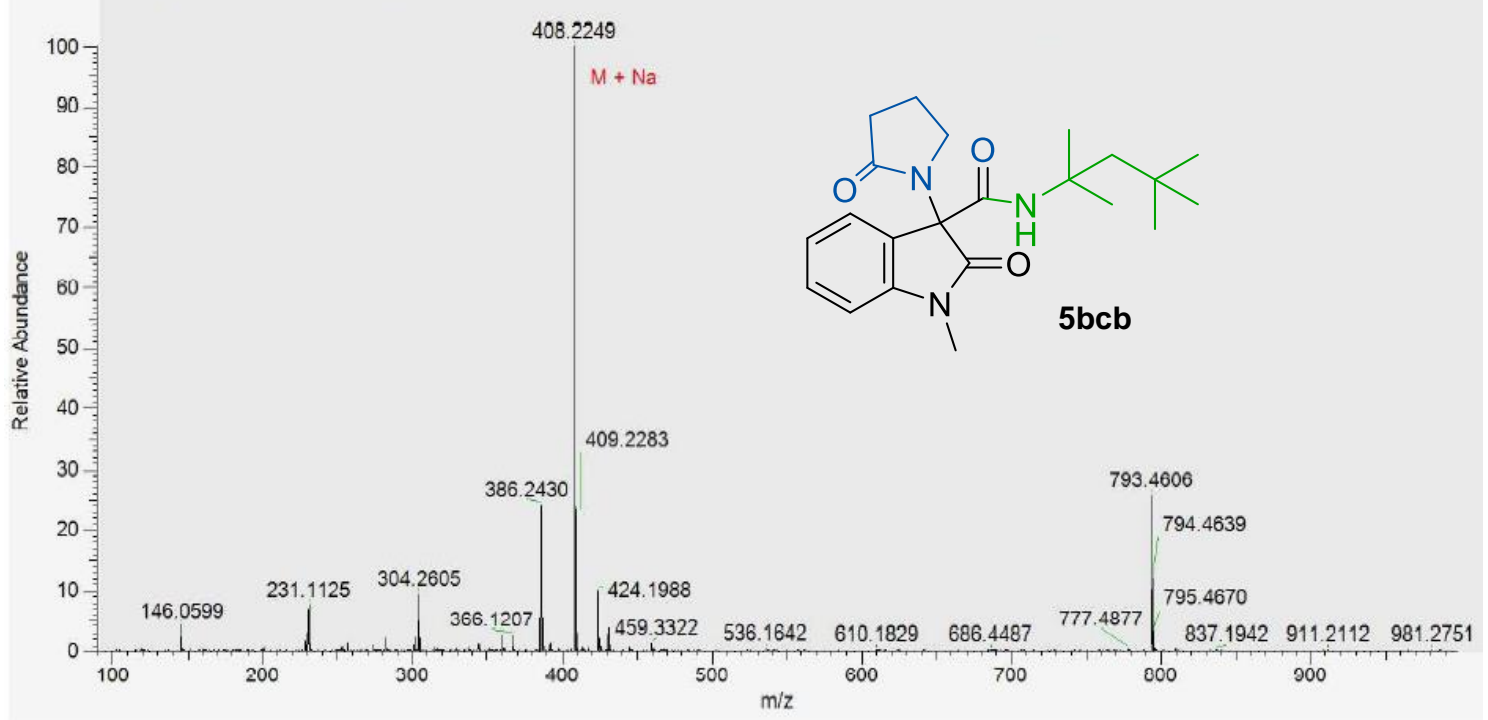

$\begin{array}{lllllll}\text { Display Formula } & \text { S Fit } & \text { RDB } & \text { Delta [ppm] } & \text { Theo. mass } & \text { Combined Score } & \text { \# Matched Iso. } \\ \mathrm{C}_{22} \mathrm{H}_{31} \mathrm{O}_{3} \mathrm{~N}_{3}{ }^{23} \mathrm{Na} & 71,3 & 8,5 & -2,23 & 408,22576 & 97,17\end{array}$

210203_071 \#15 RT: 0.14 AV: 1 NL: $4.47 E+008$

T: FTMS + p ESI Full ms [100.0000-1500.0000]

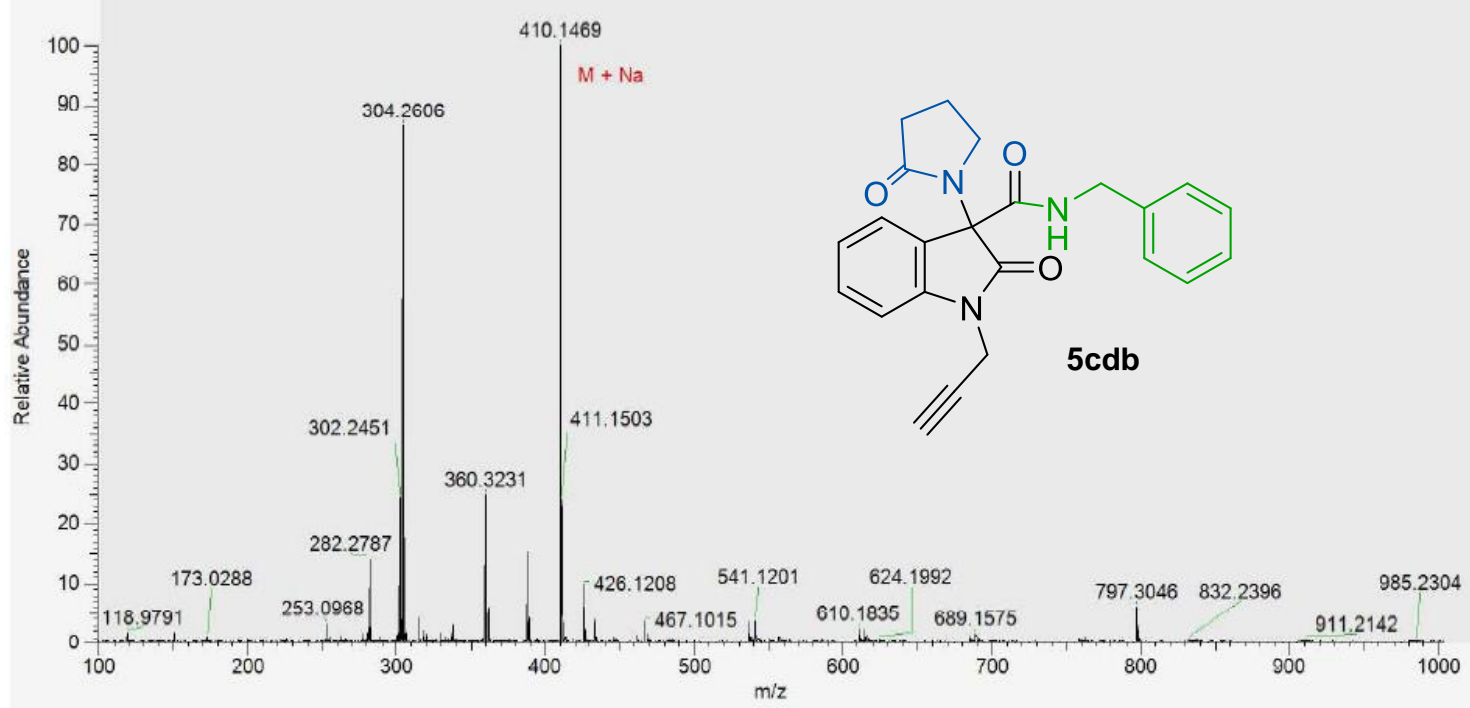

$\begin{array}{lllllll}\text { Display Formula } & \text { S Fit } & \text { RDB } & \text { Delta [ppm] } & \text { Theo. mass } & \text { Combined Score } & \text { \# Matched Iso. } \\ \mathrm{C}_{23} \mathrm{H}_{21} \mathrm{O}_{3} \mathrm{~N}_{3}{ }^{23} \mathrm{Na} & 60,43 & 14,5 & -1,61 & 410,14751 & 95,44\end{array}$




\section{ATR-FTIR Spectra}

\subsection{ATR-FTIR Spectra of $\beta$-lactam-oxindole hybrids}

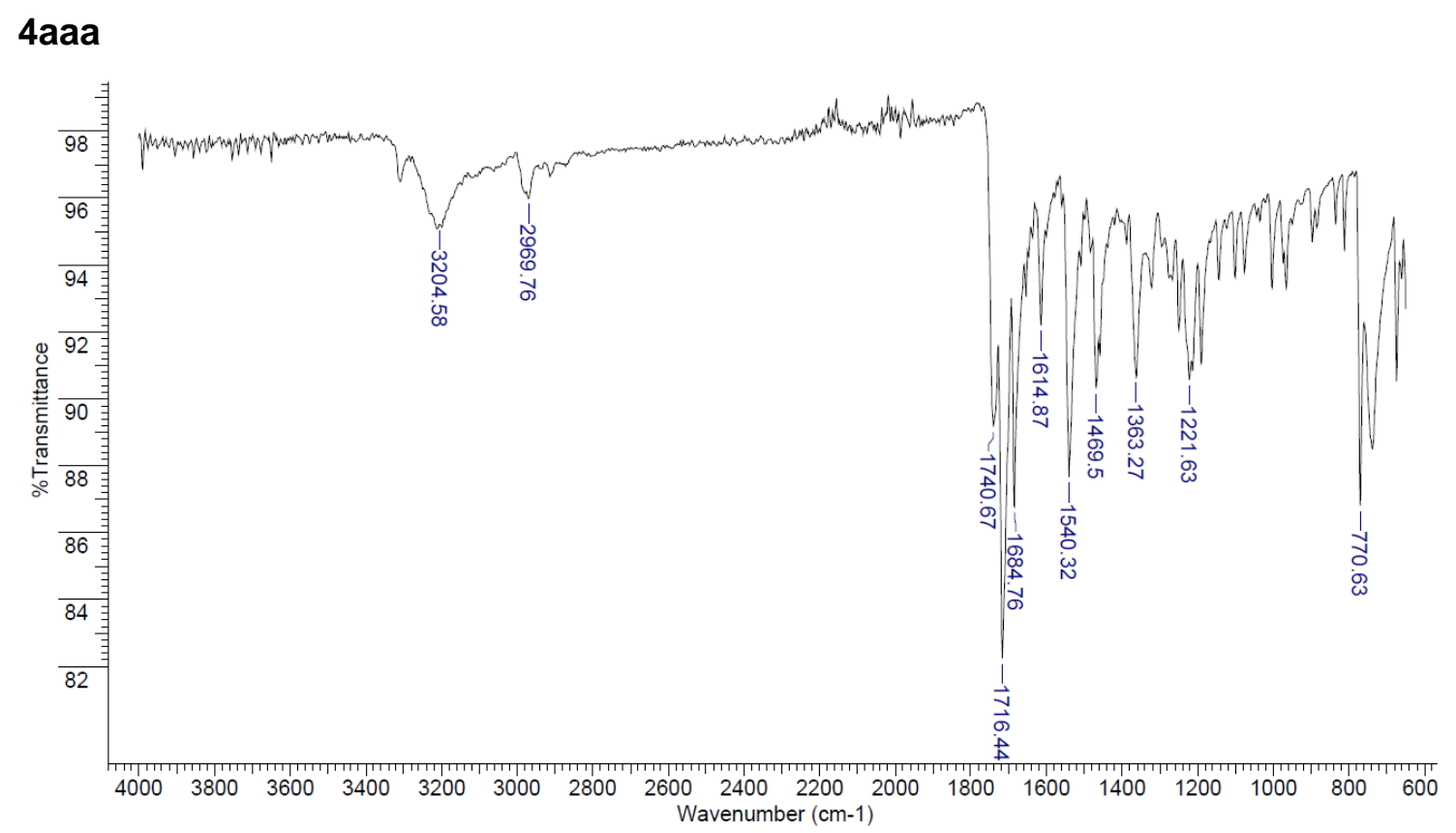

\section{4baa}

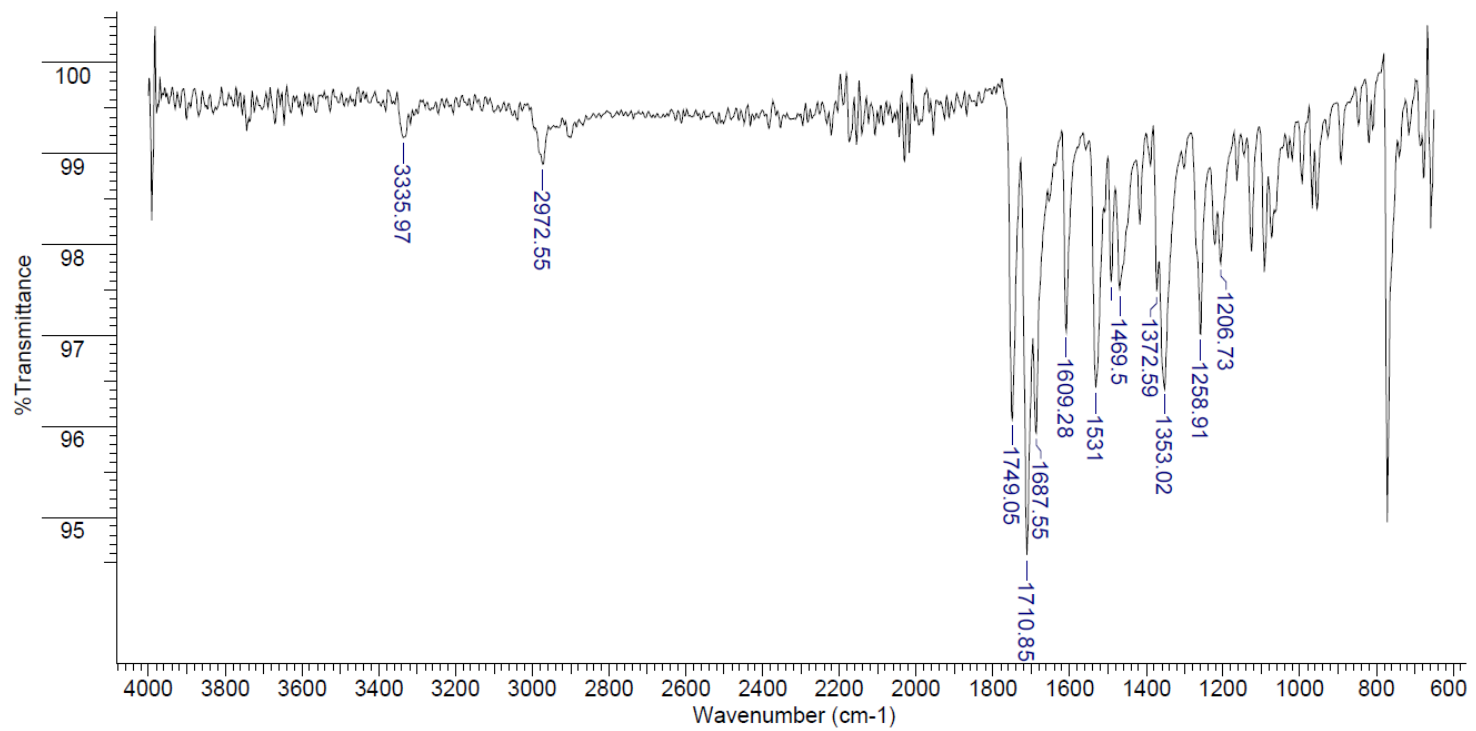


4caa

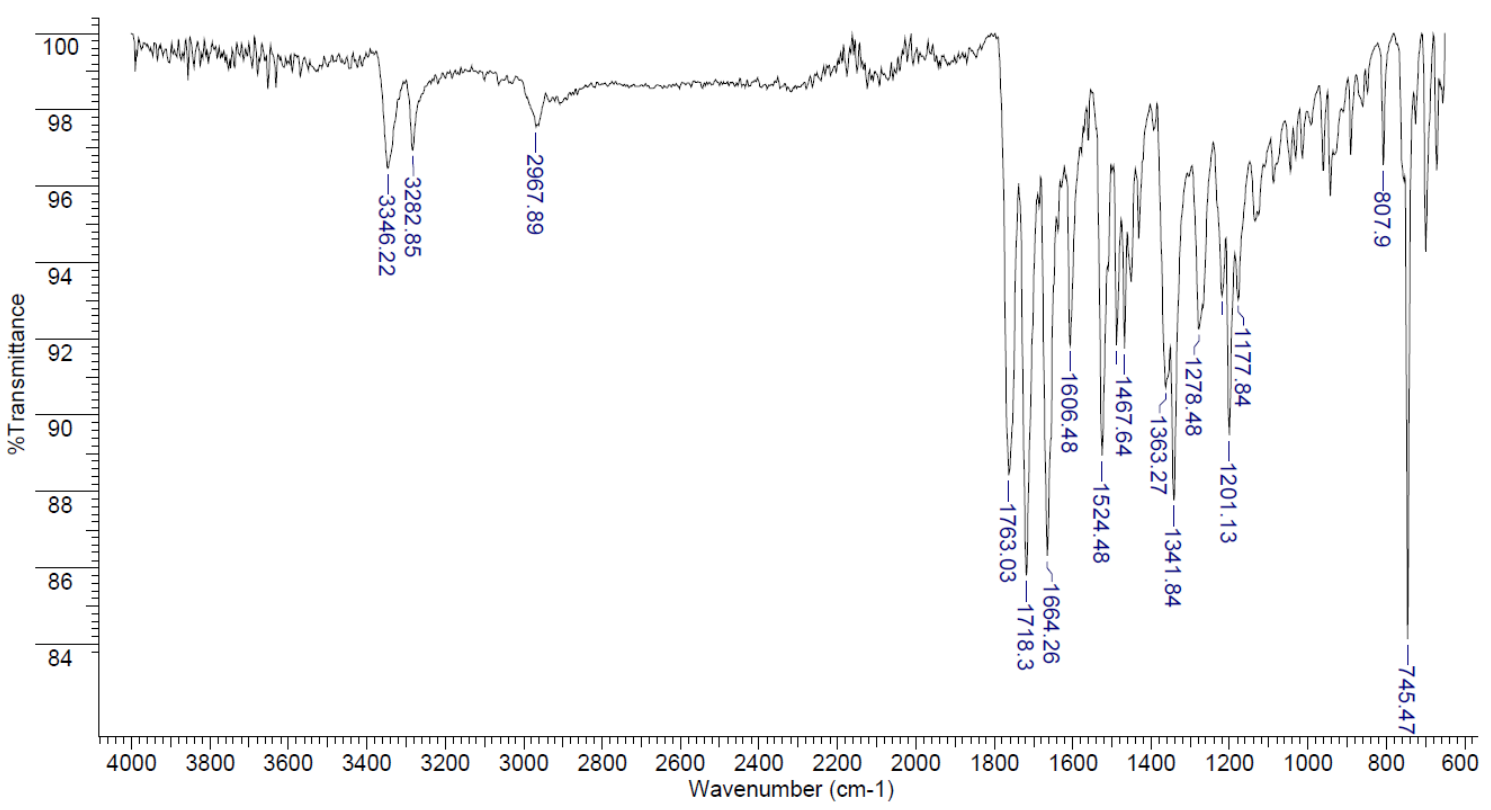

\section{4daa}

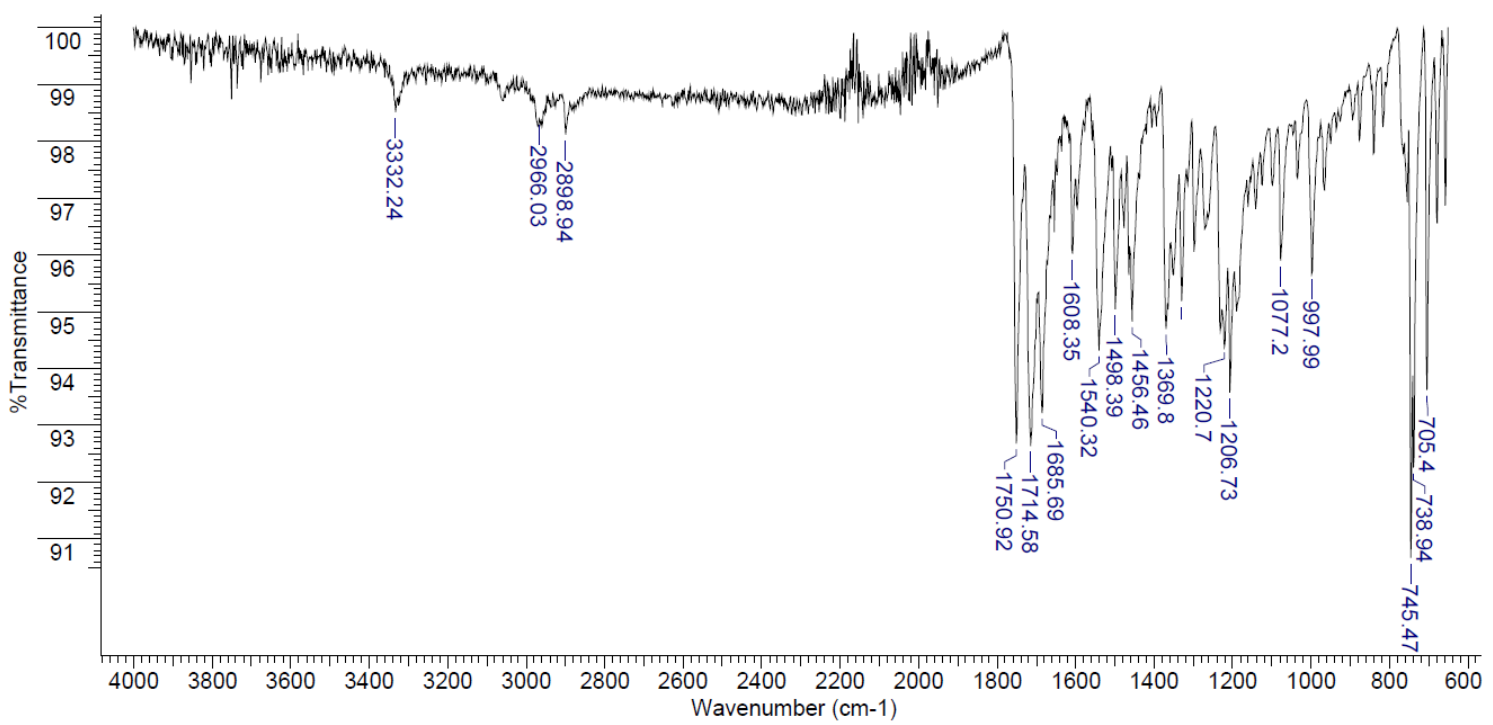




\section{4eaa}

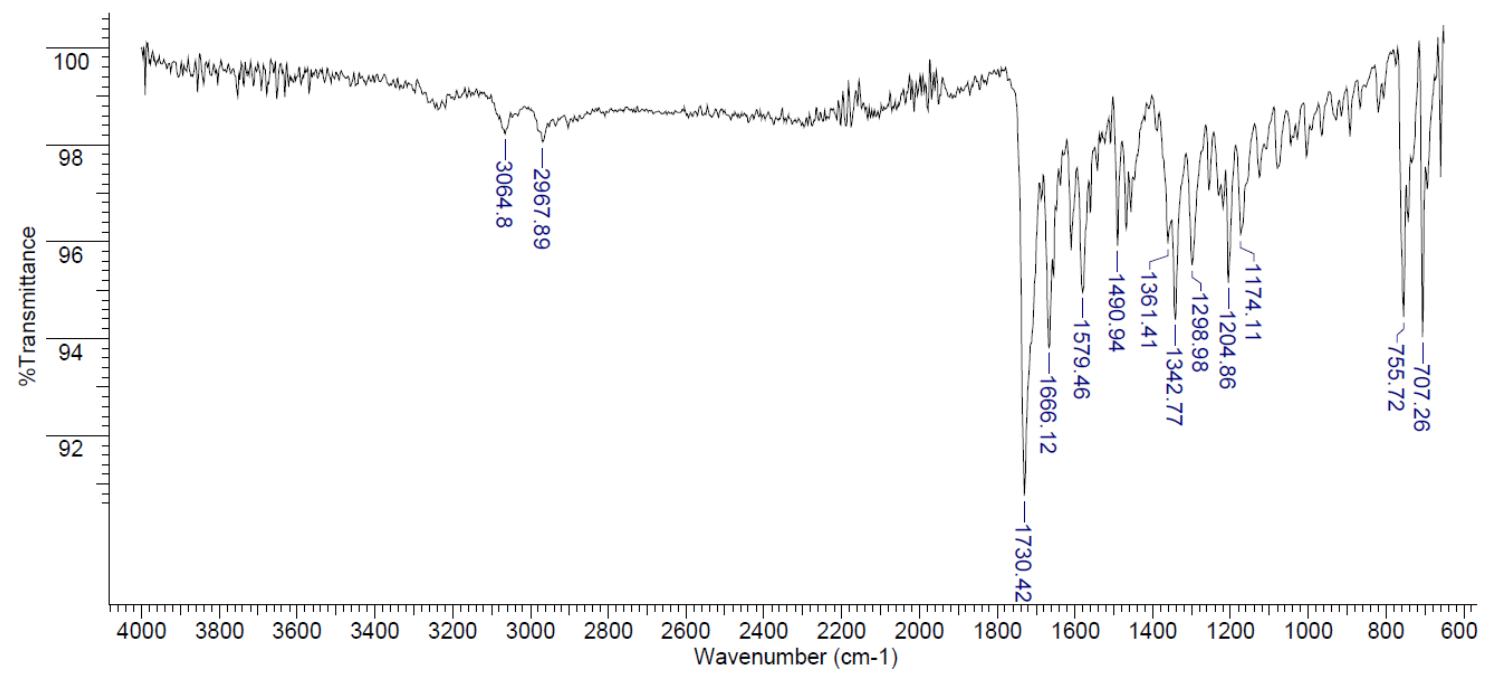

\section{4faa}

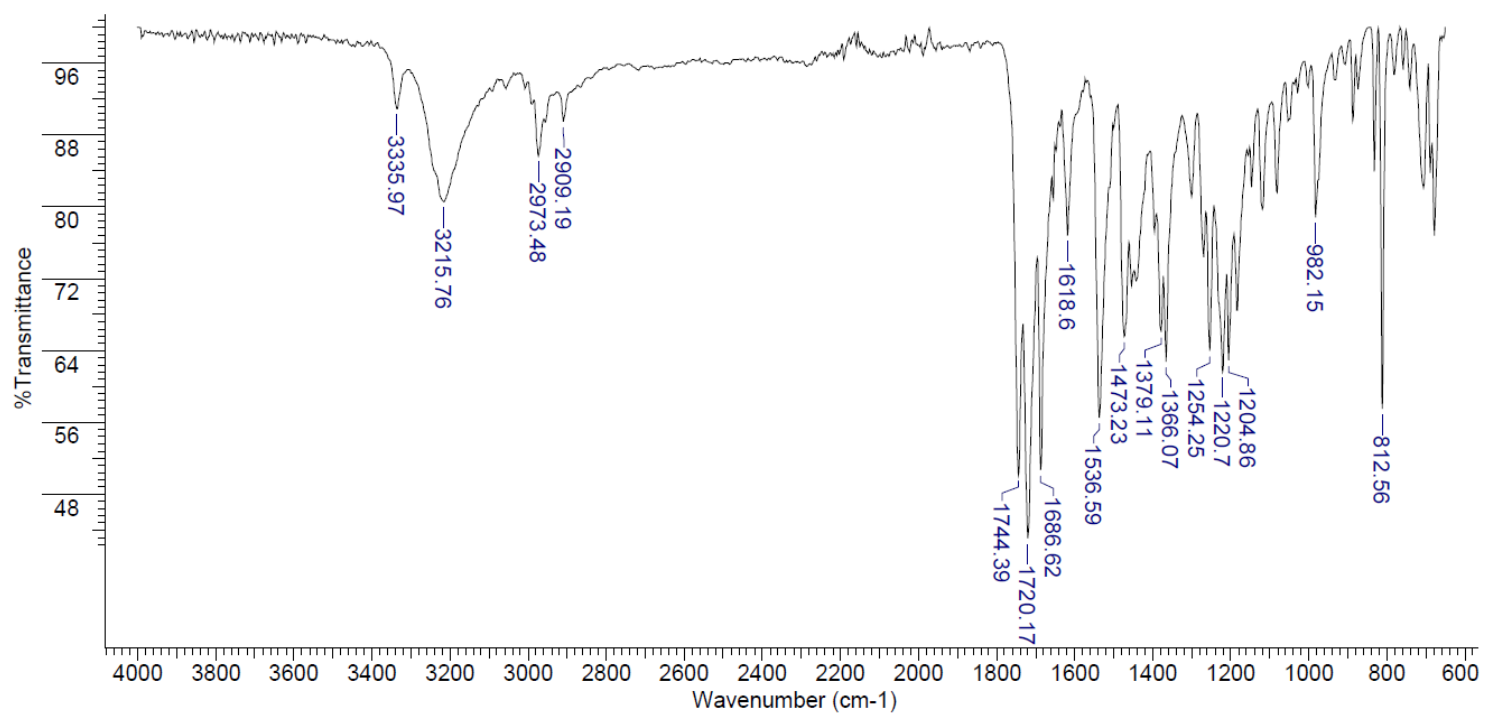




\section{4gaa}

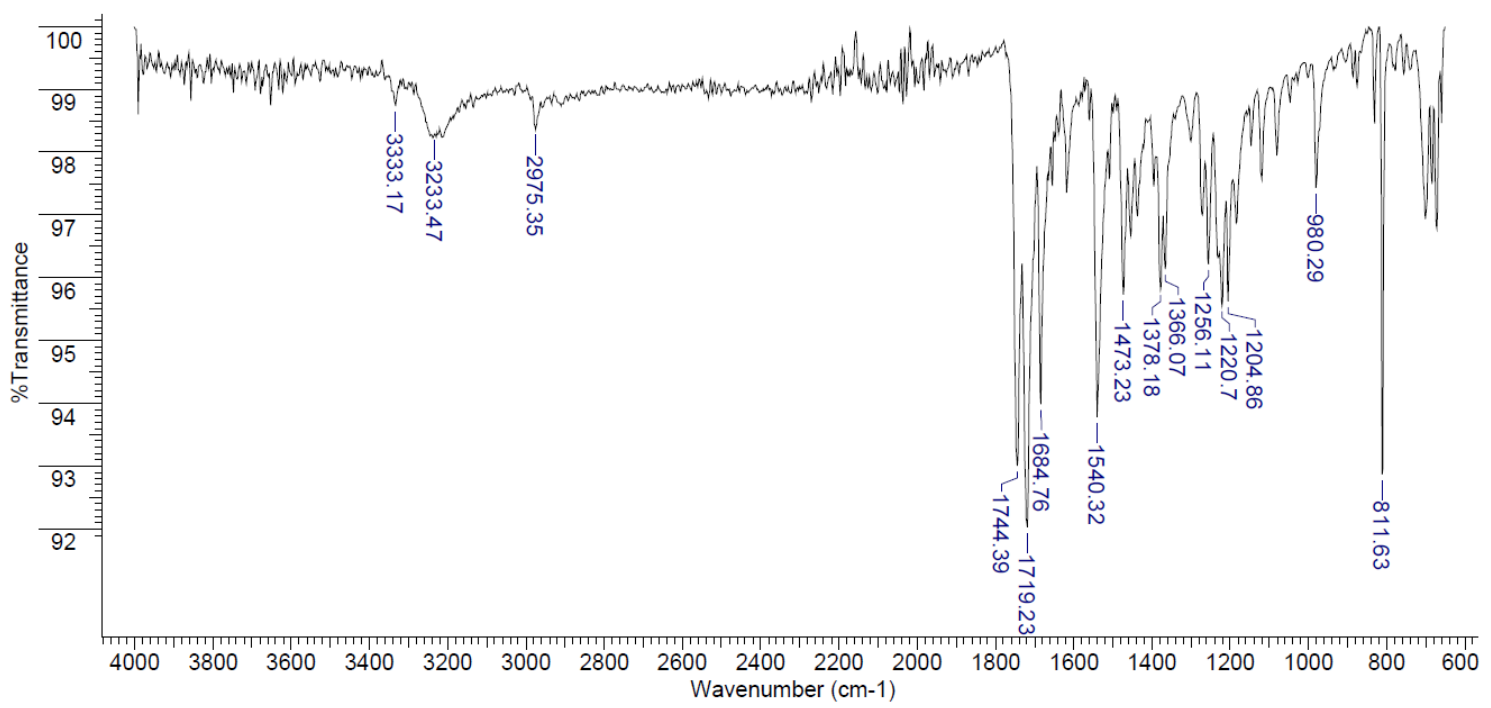

\section{4haa}

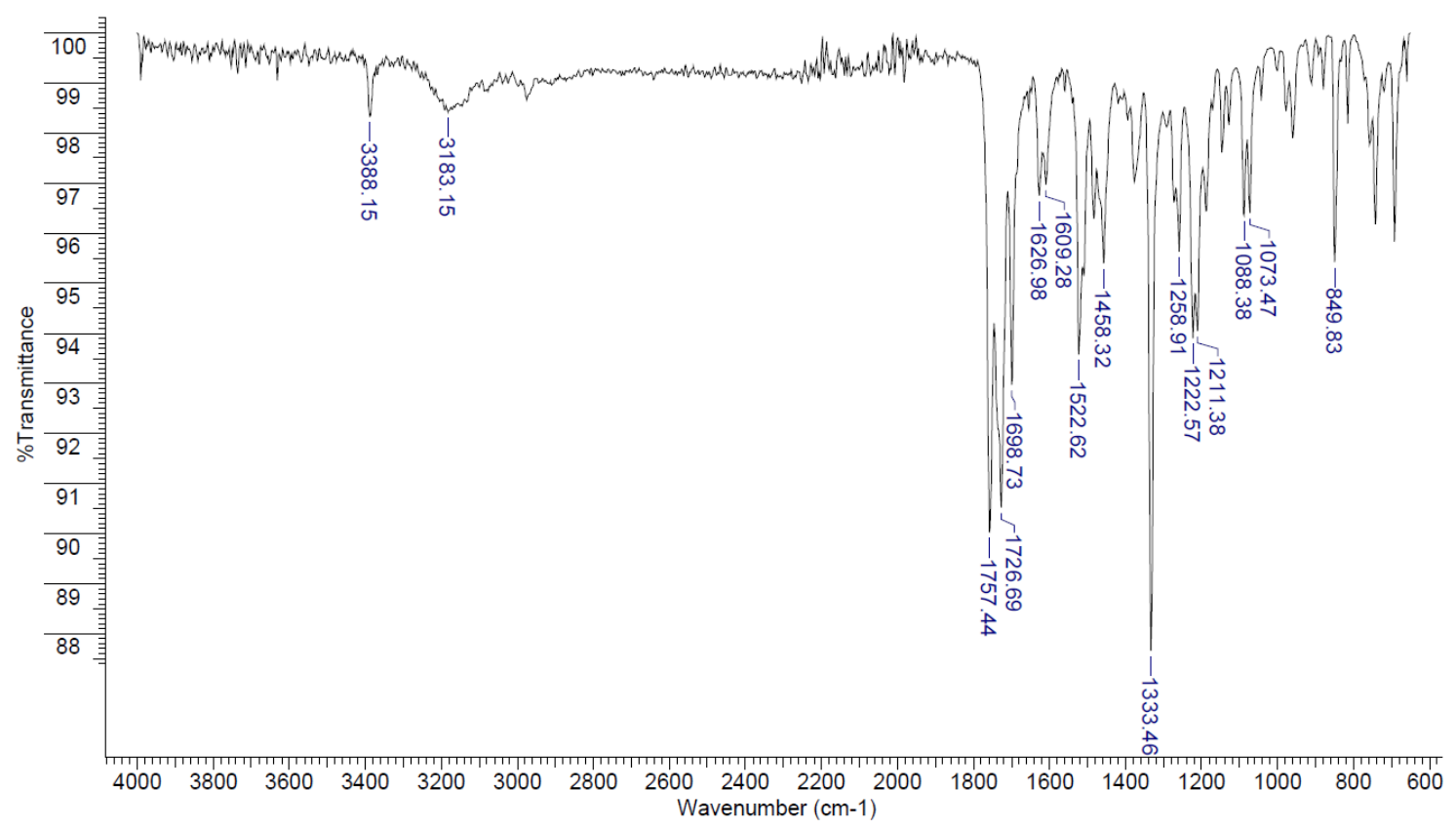




\section{$4 i a a$}

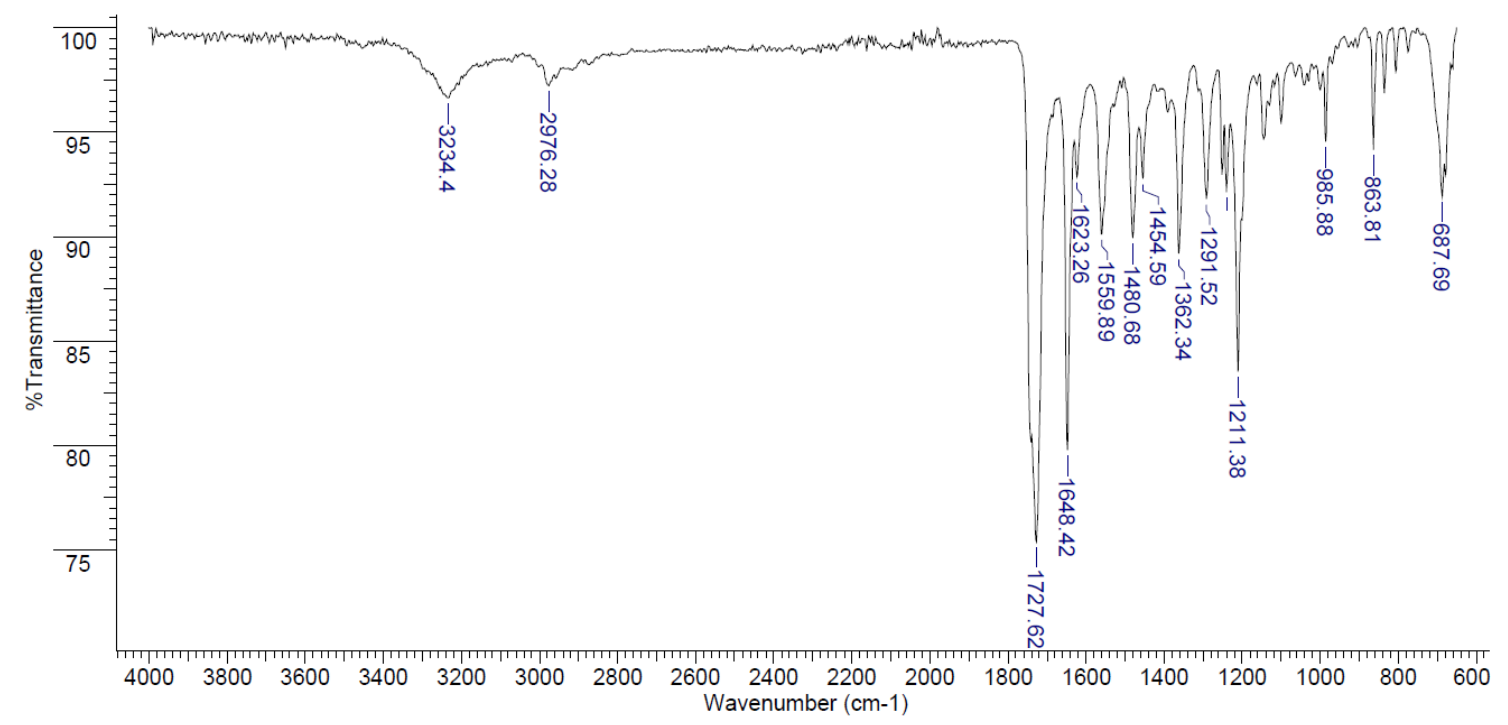

\section{4jaa}

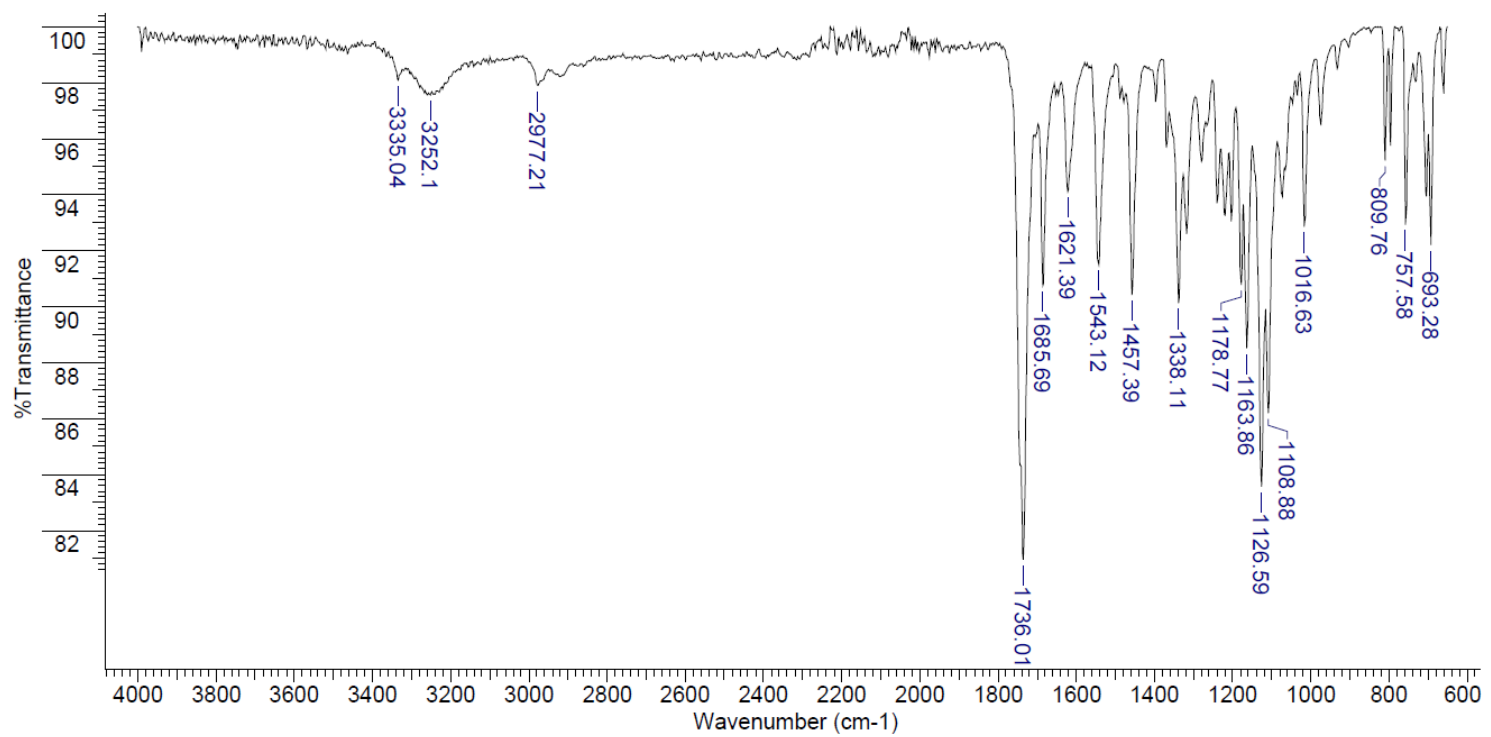


4aba

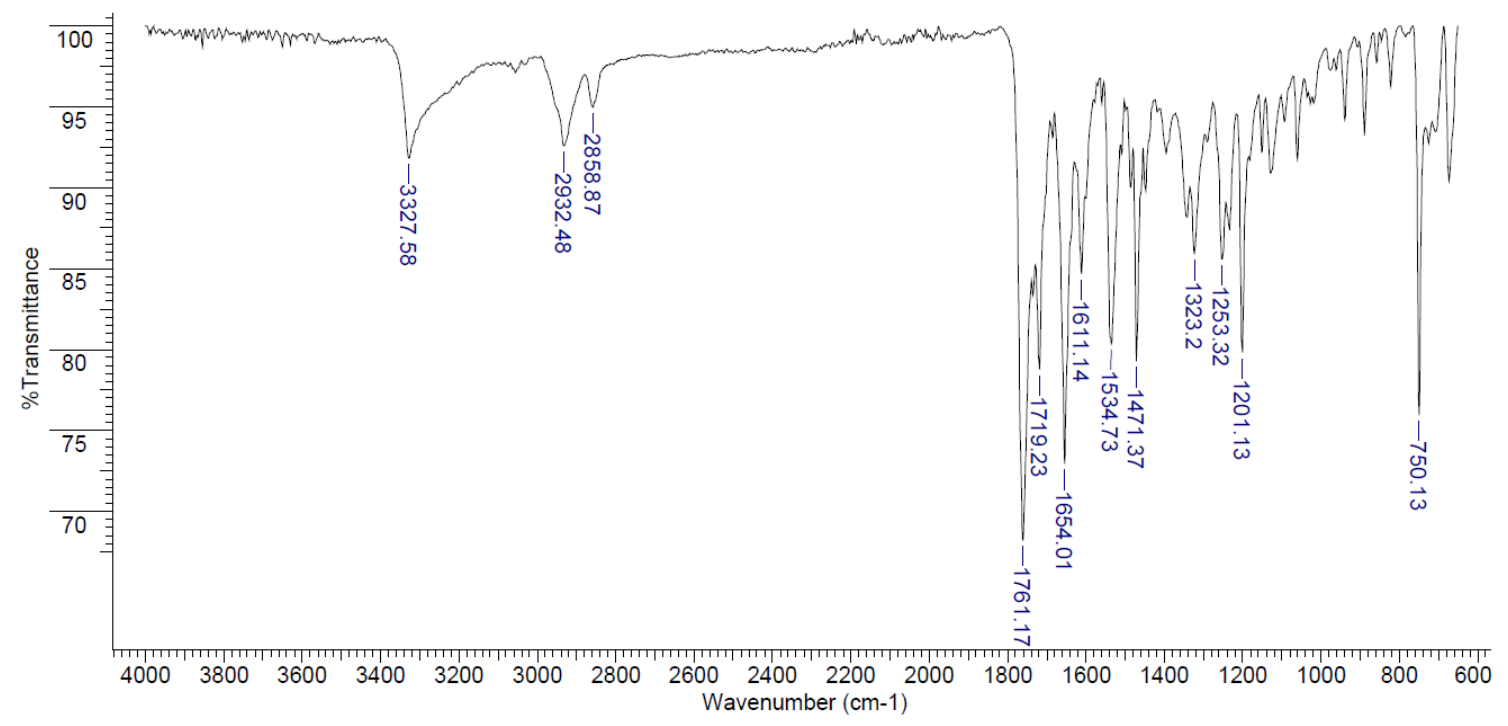

\section{4aca}

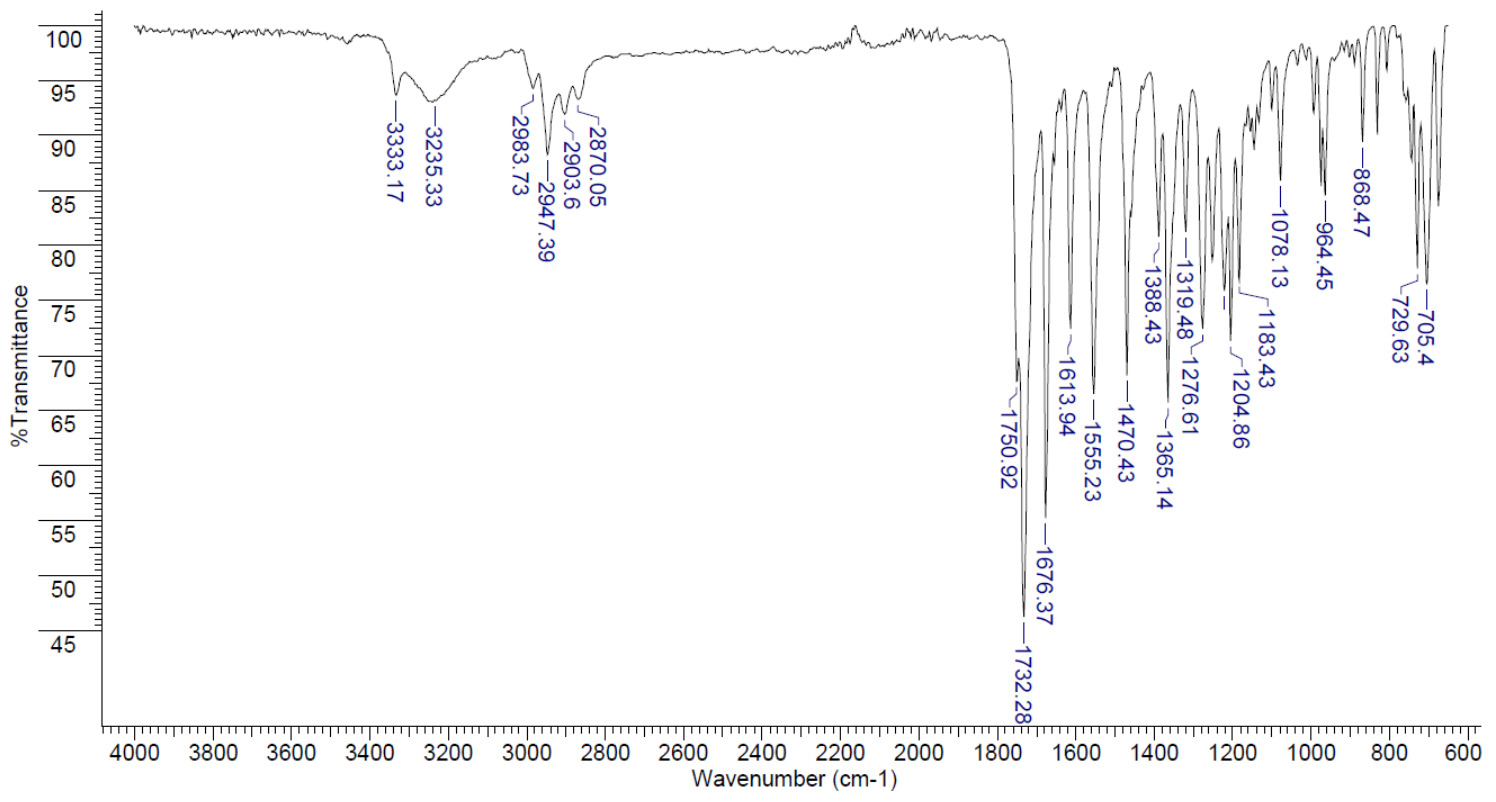




\section{4ada}

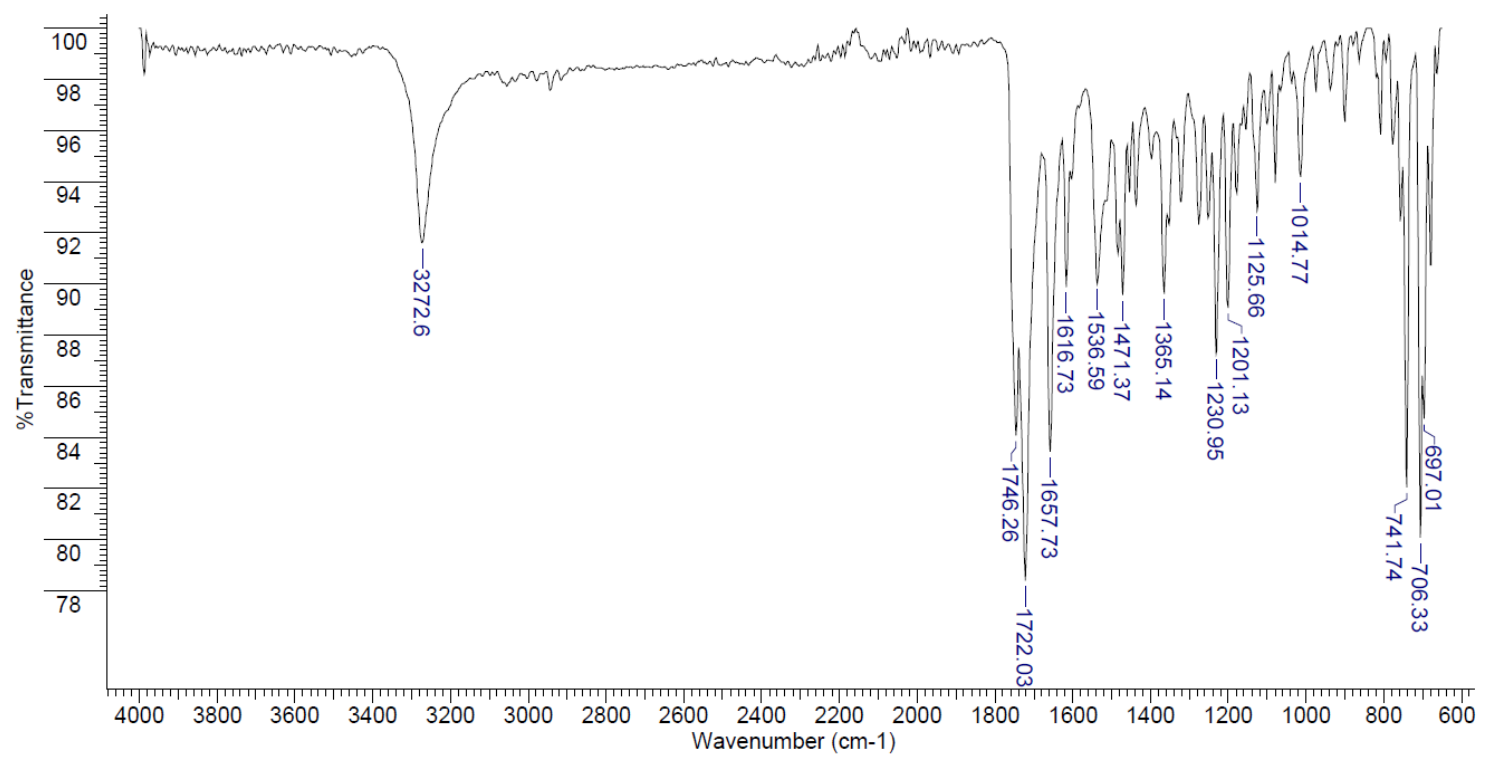

\section{4eca}

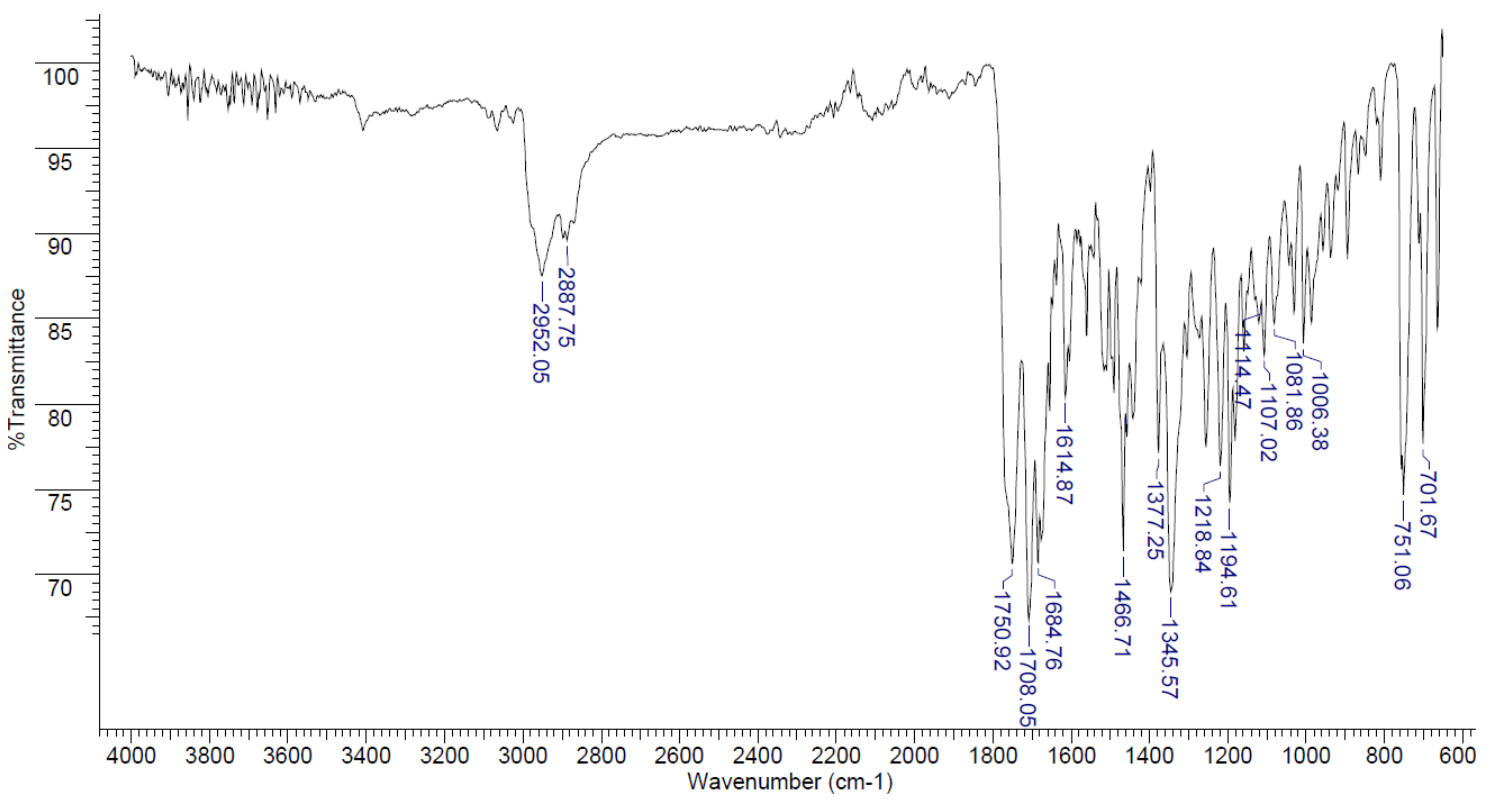




\section{4cda}

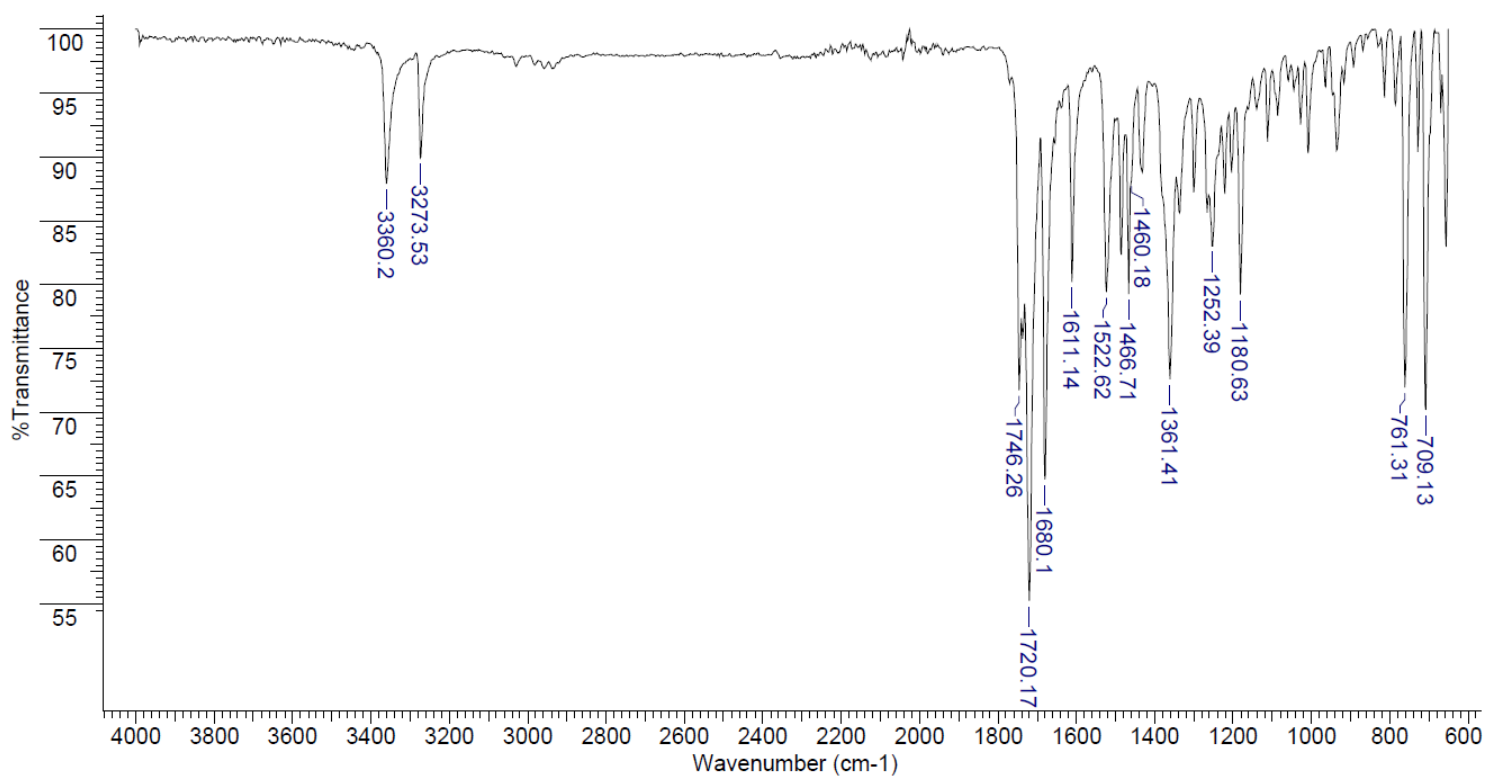

\section{4bba}

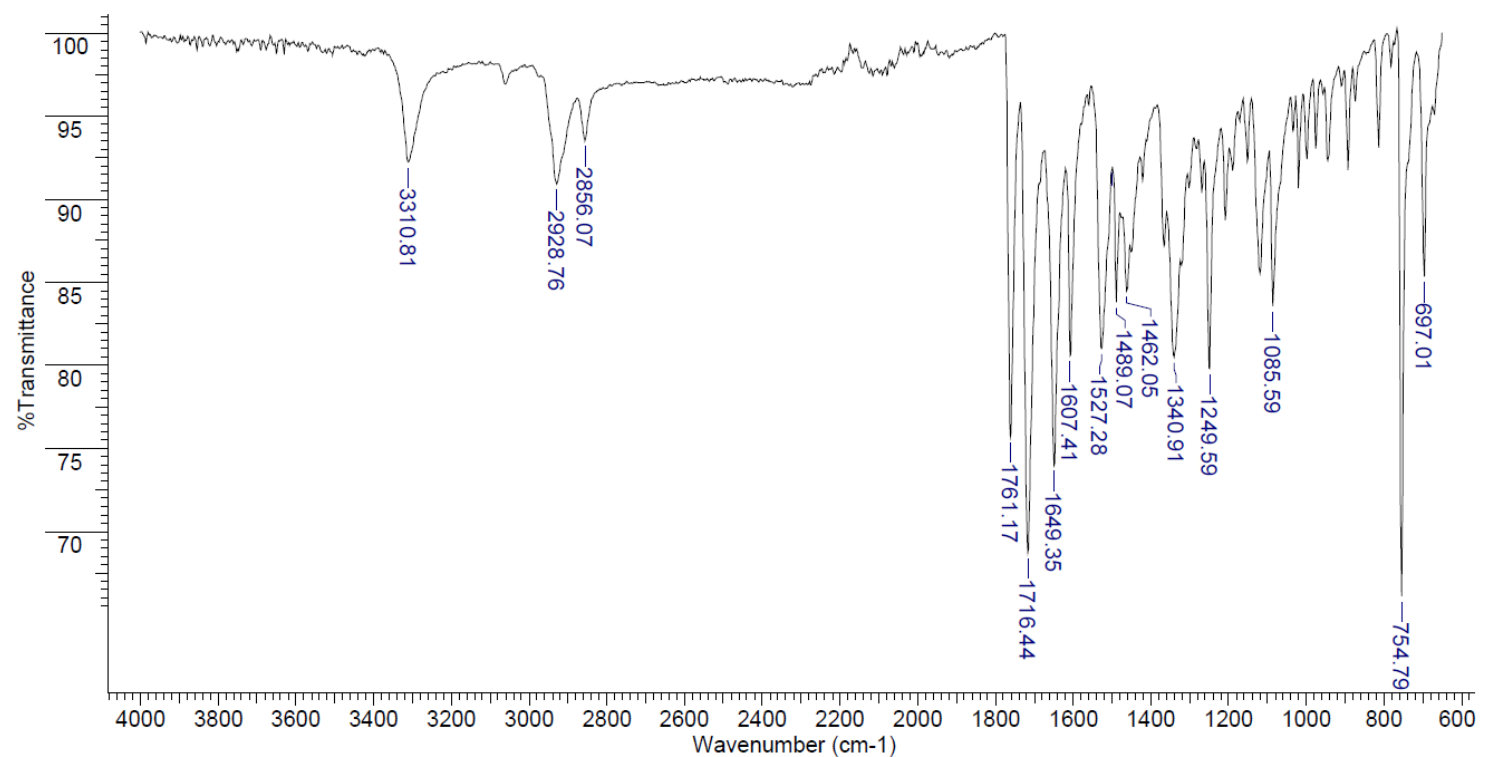




\section{$4 b c a$}

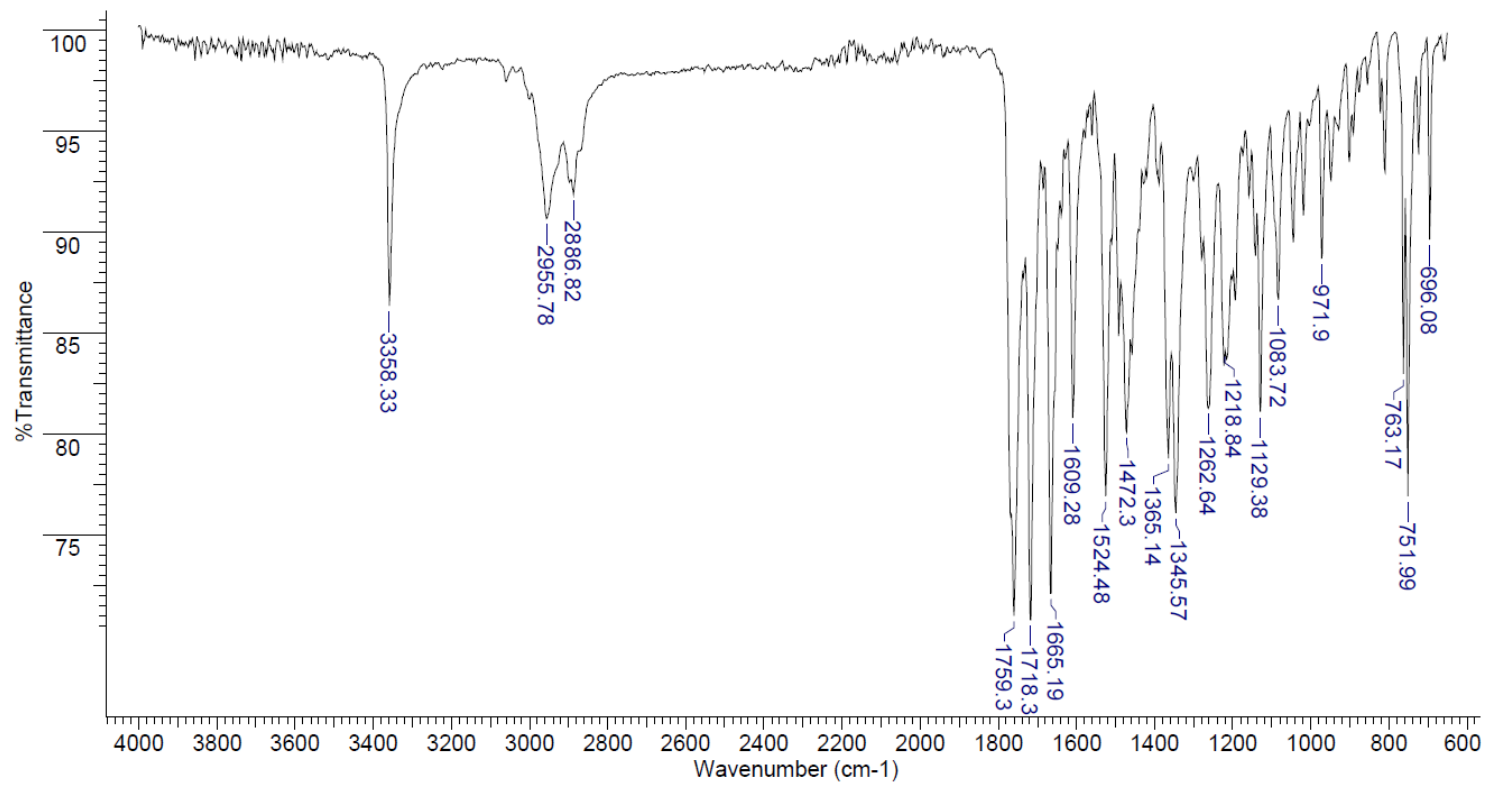




\subsection{ATR-FTIR Spectra of $y$-lactam-oxindole hybrids}

\section{$5 a a b$}

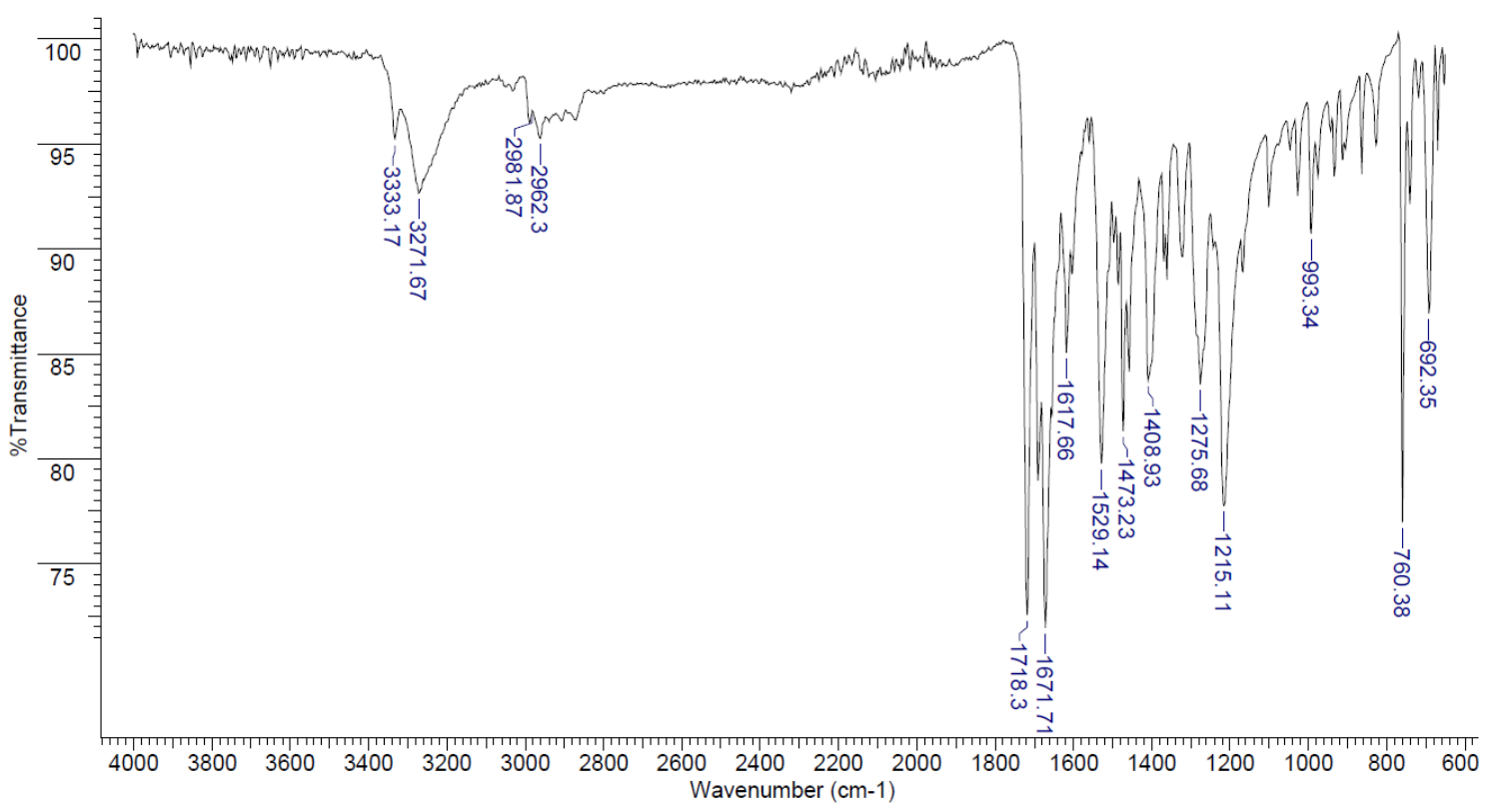

\section{$5 b a b$}

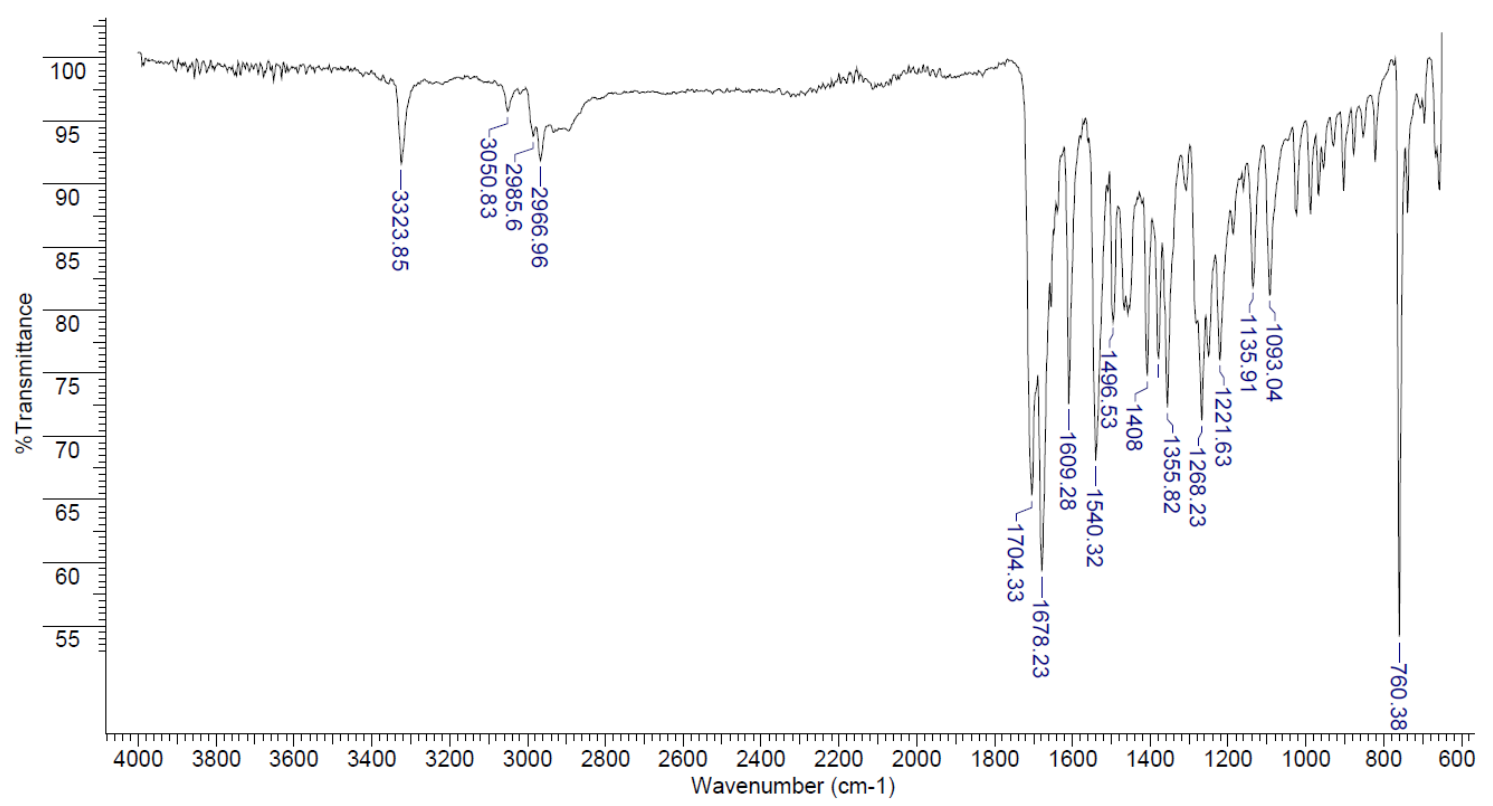




\section{$5 d a b$}

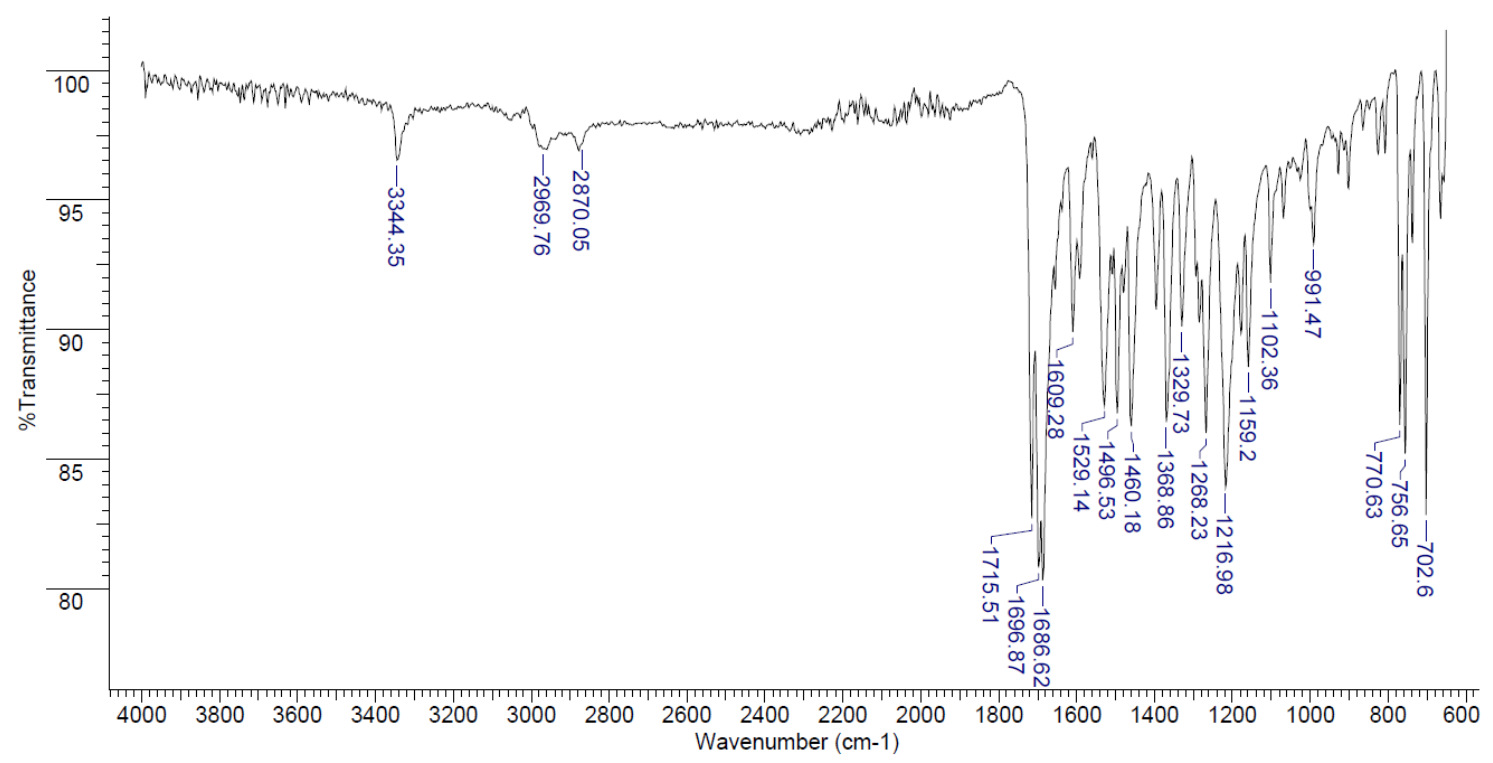

\section{$5 e a b$}

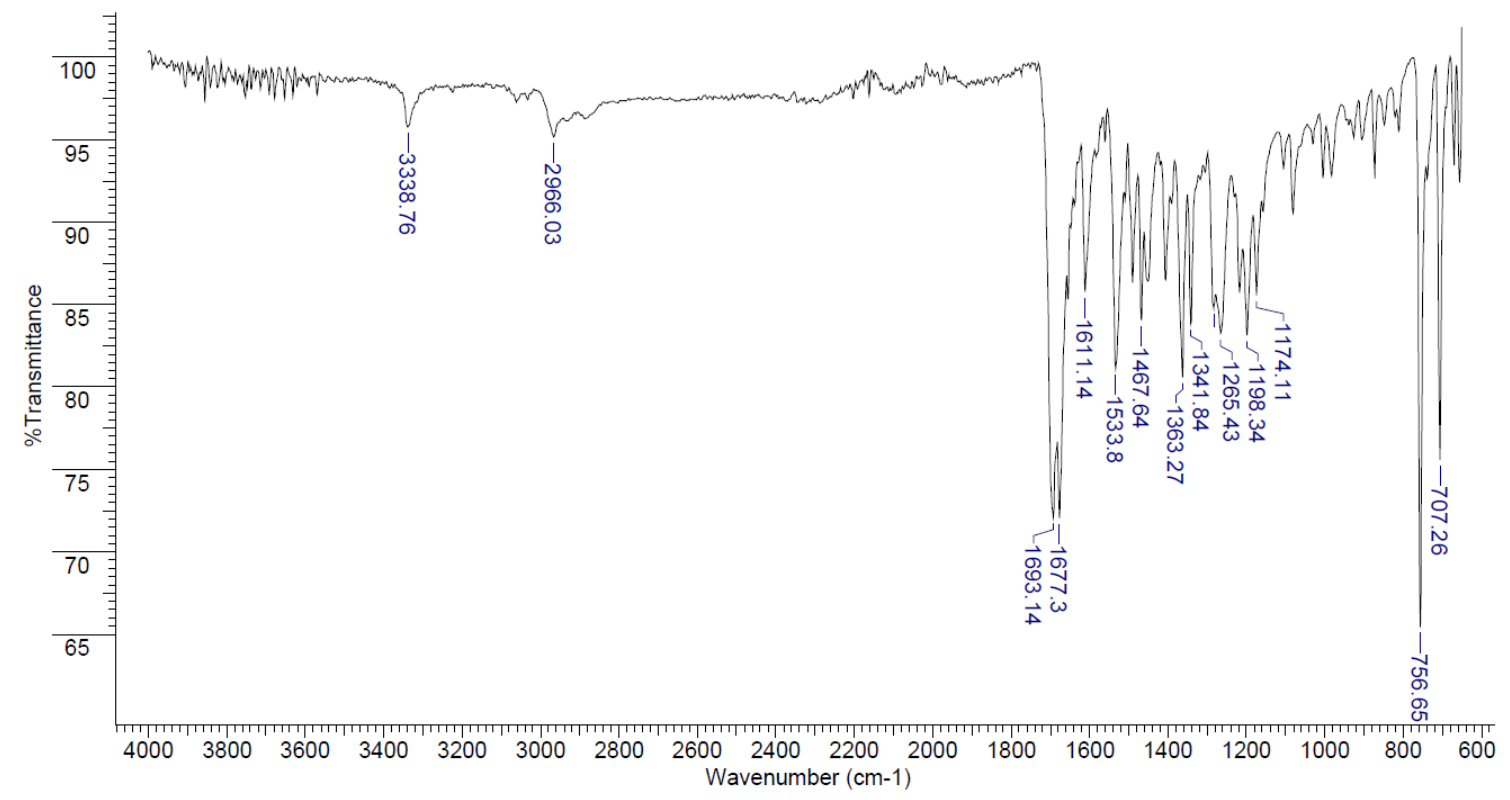


$5 f a b$

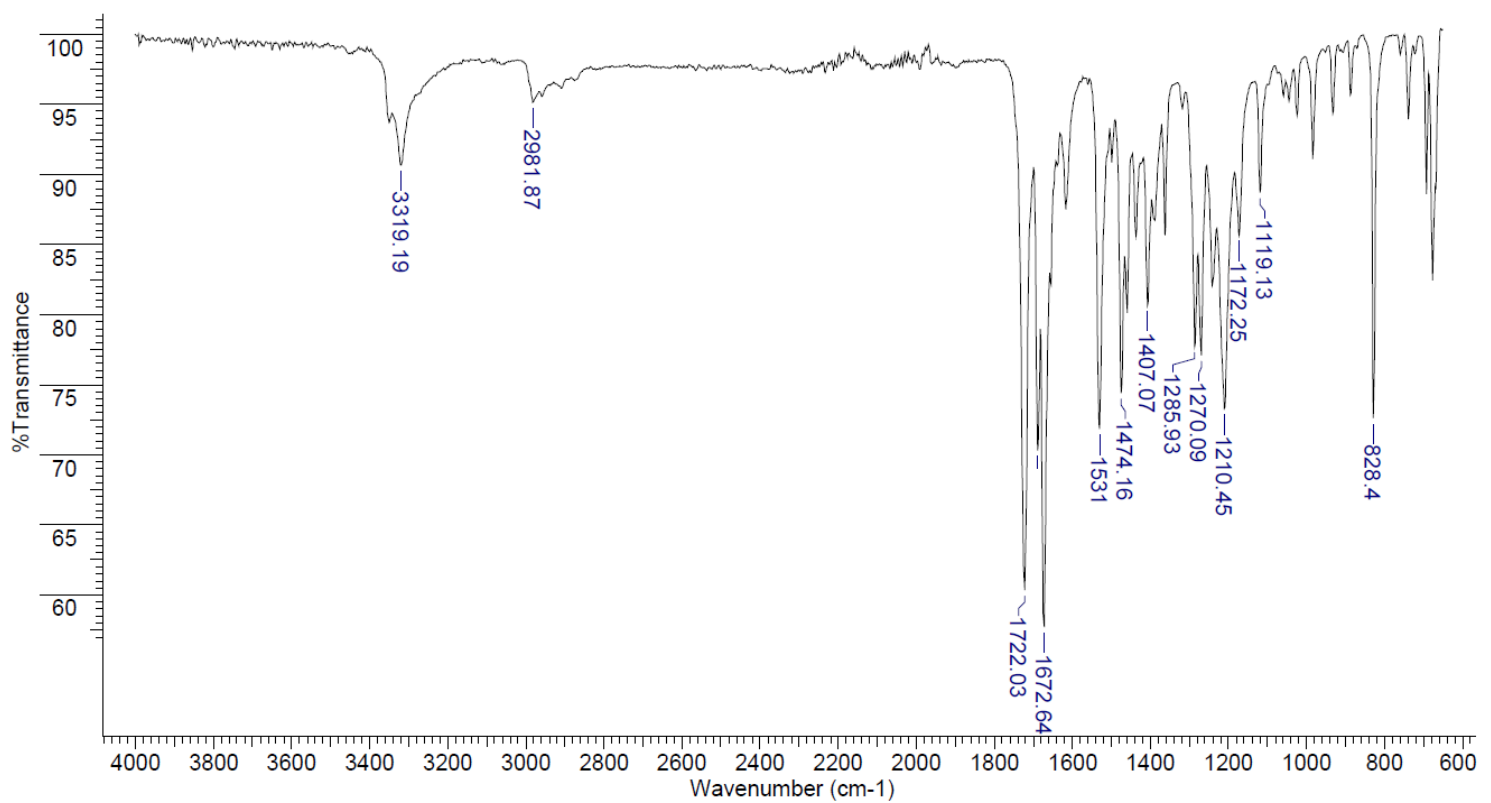

\section{$5 g a b$}

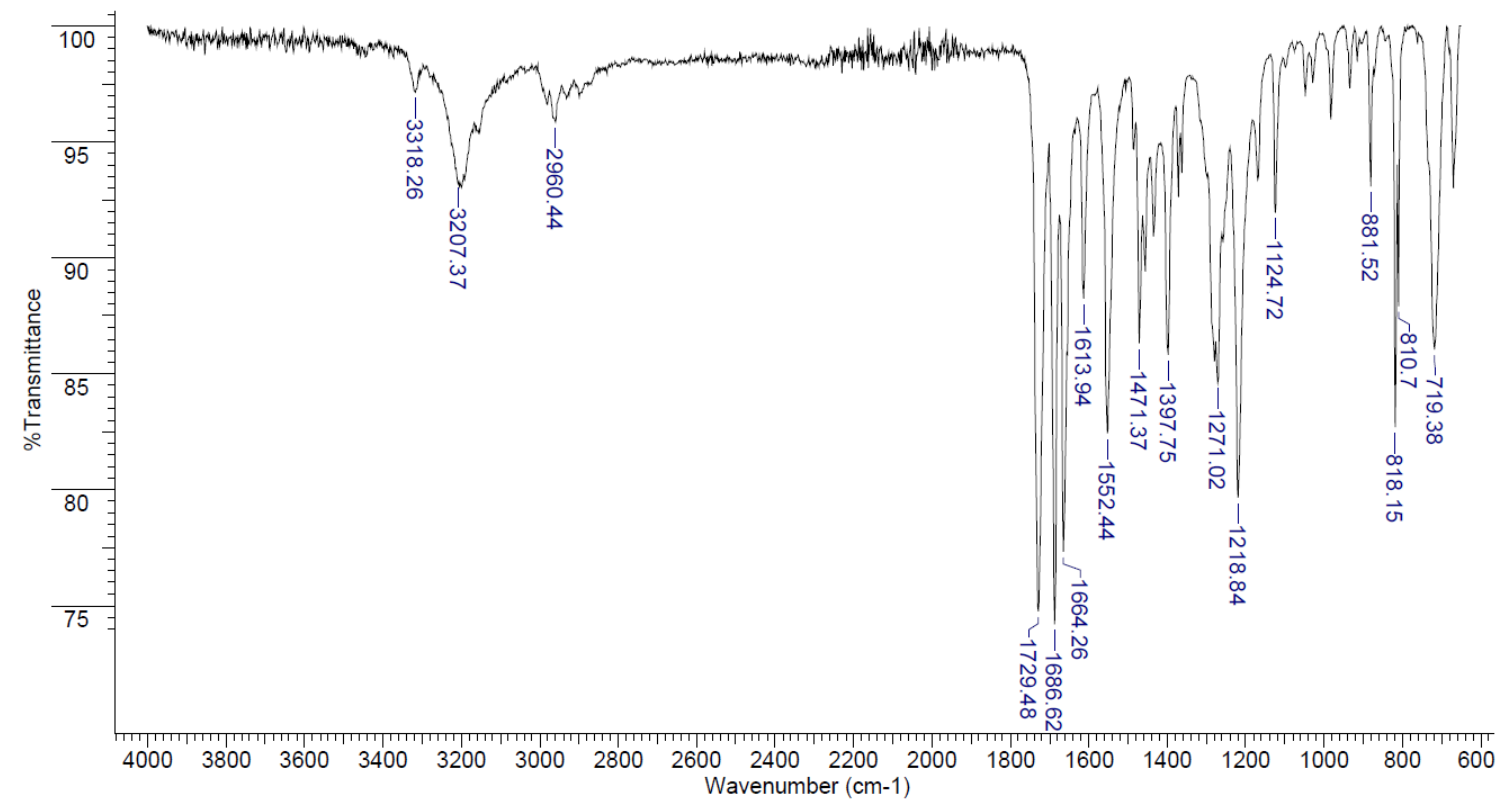




\section{5hab}

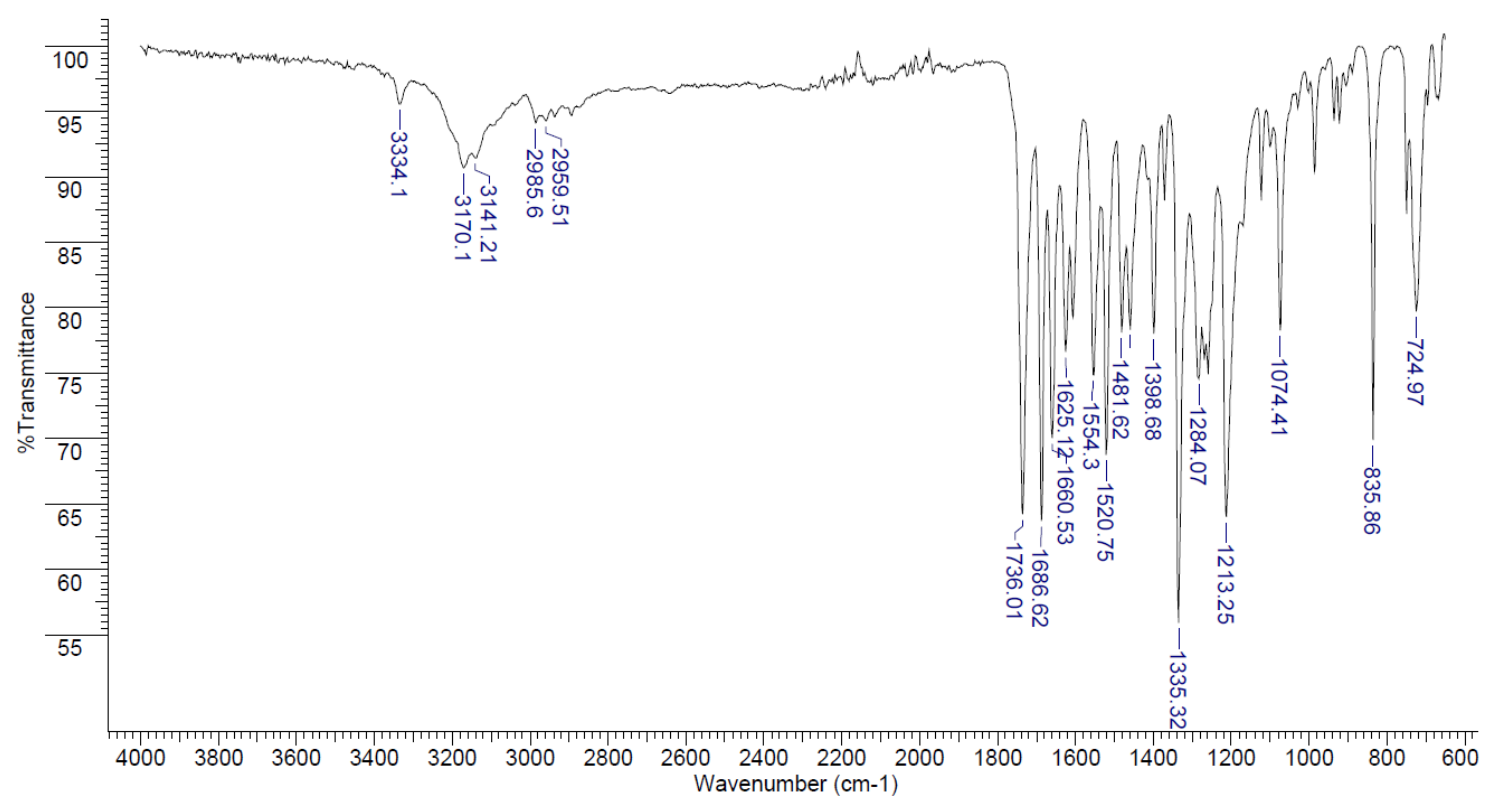

\section{$5 i a b$}

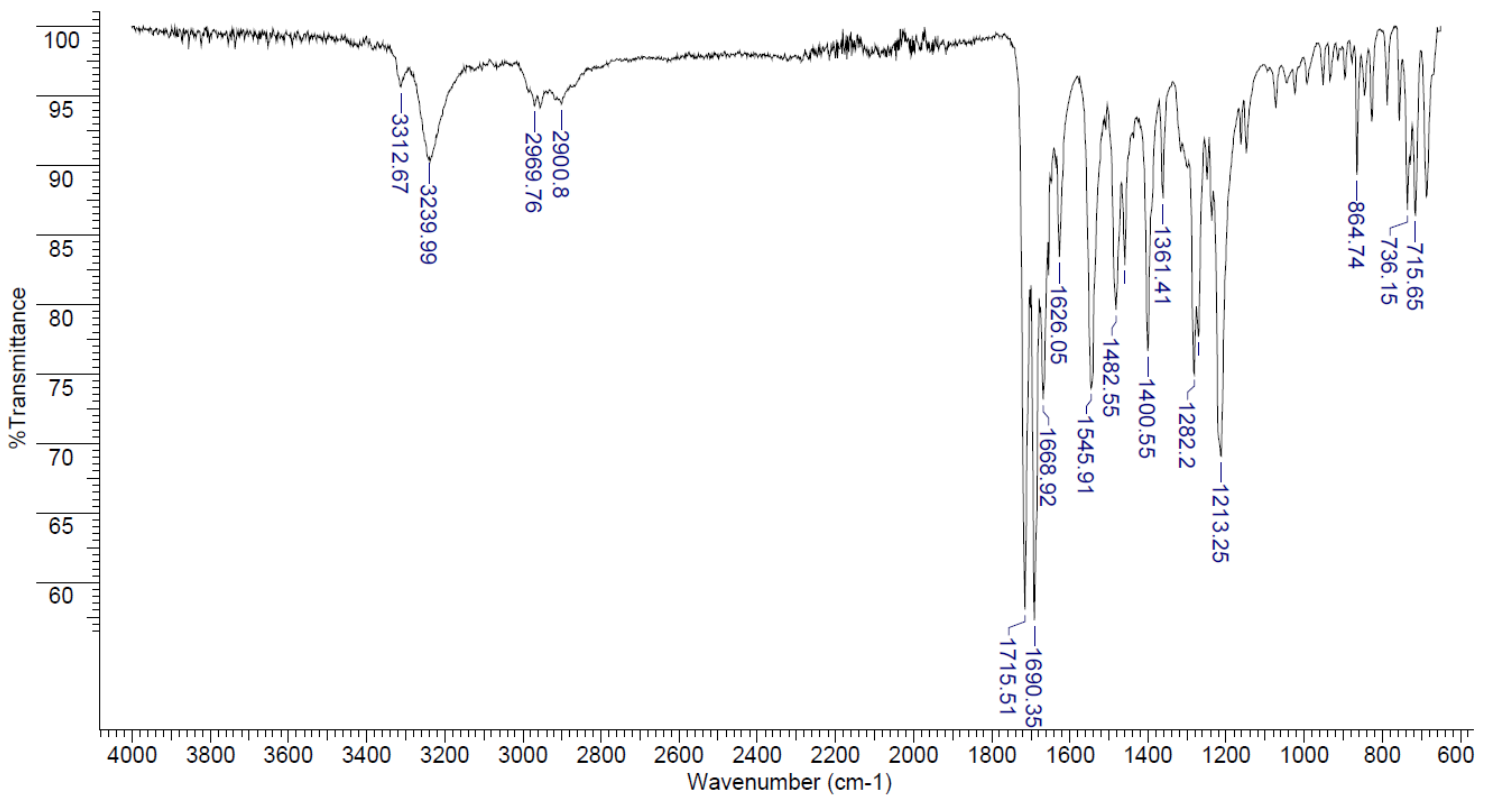




\section{$5 j a b$}

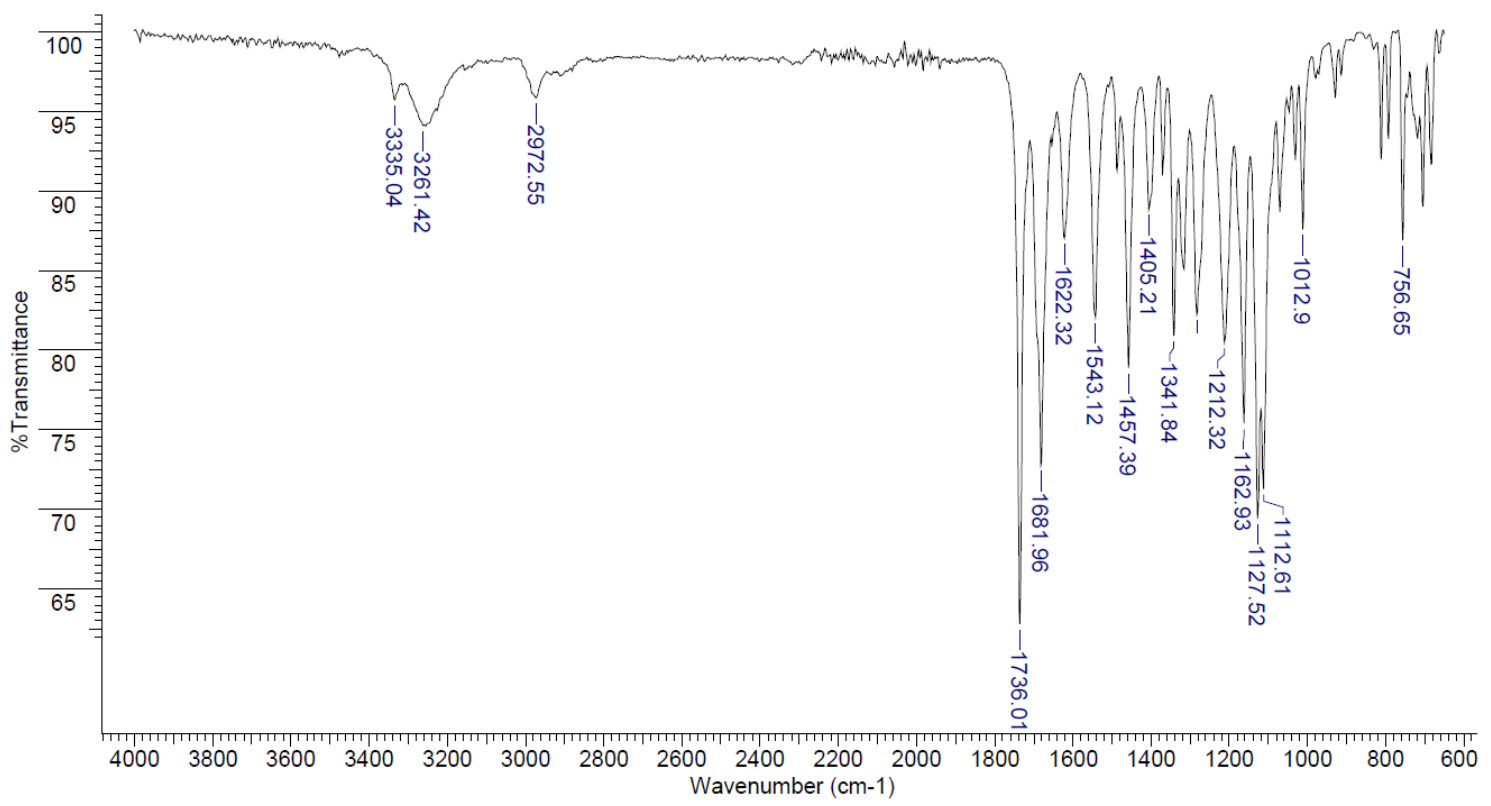

\section{$5 a b b$}

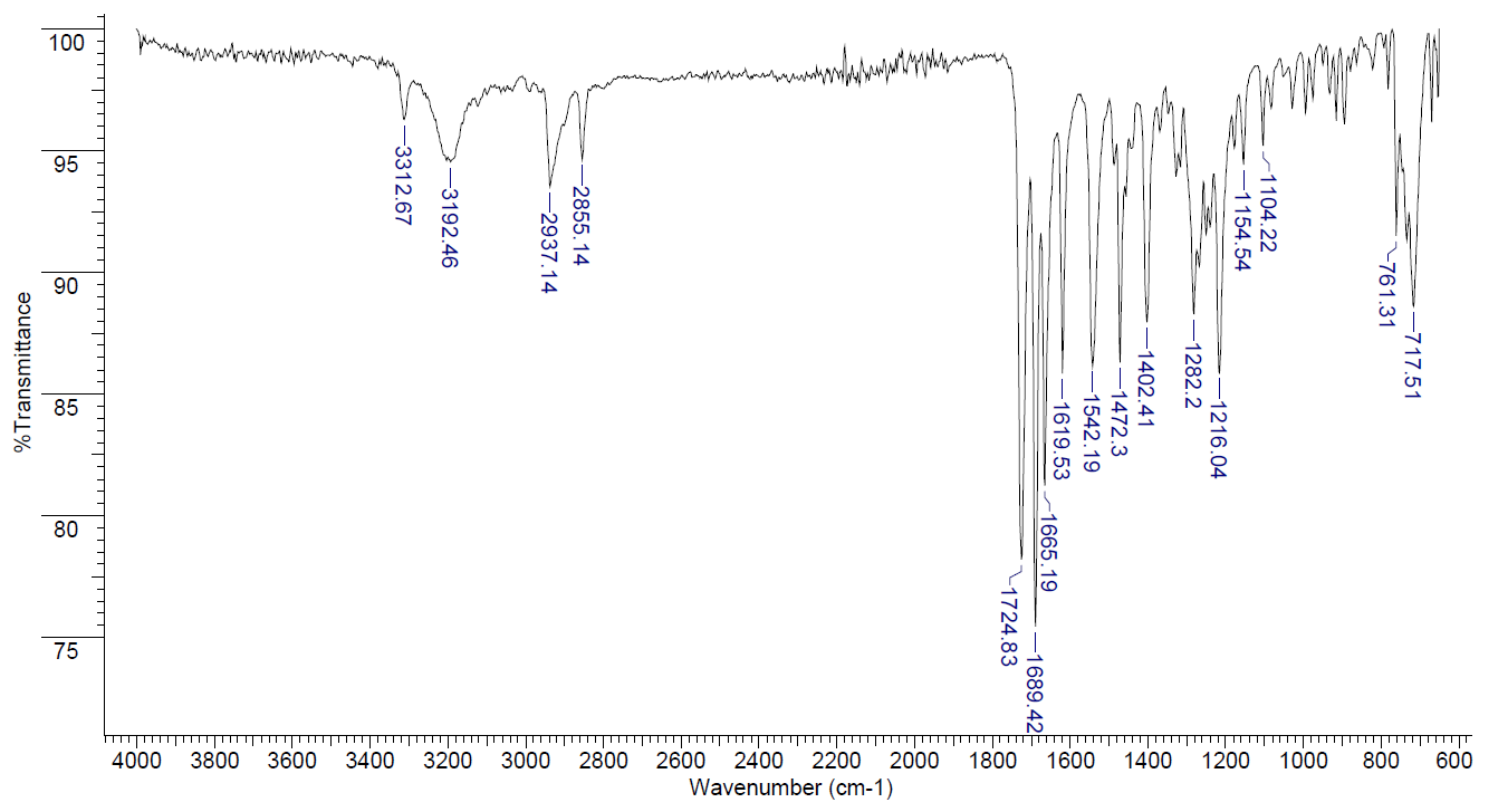


$5 a c b$

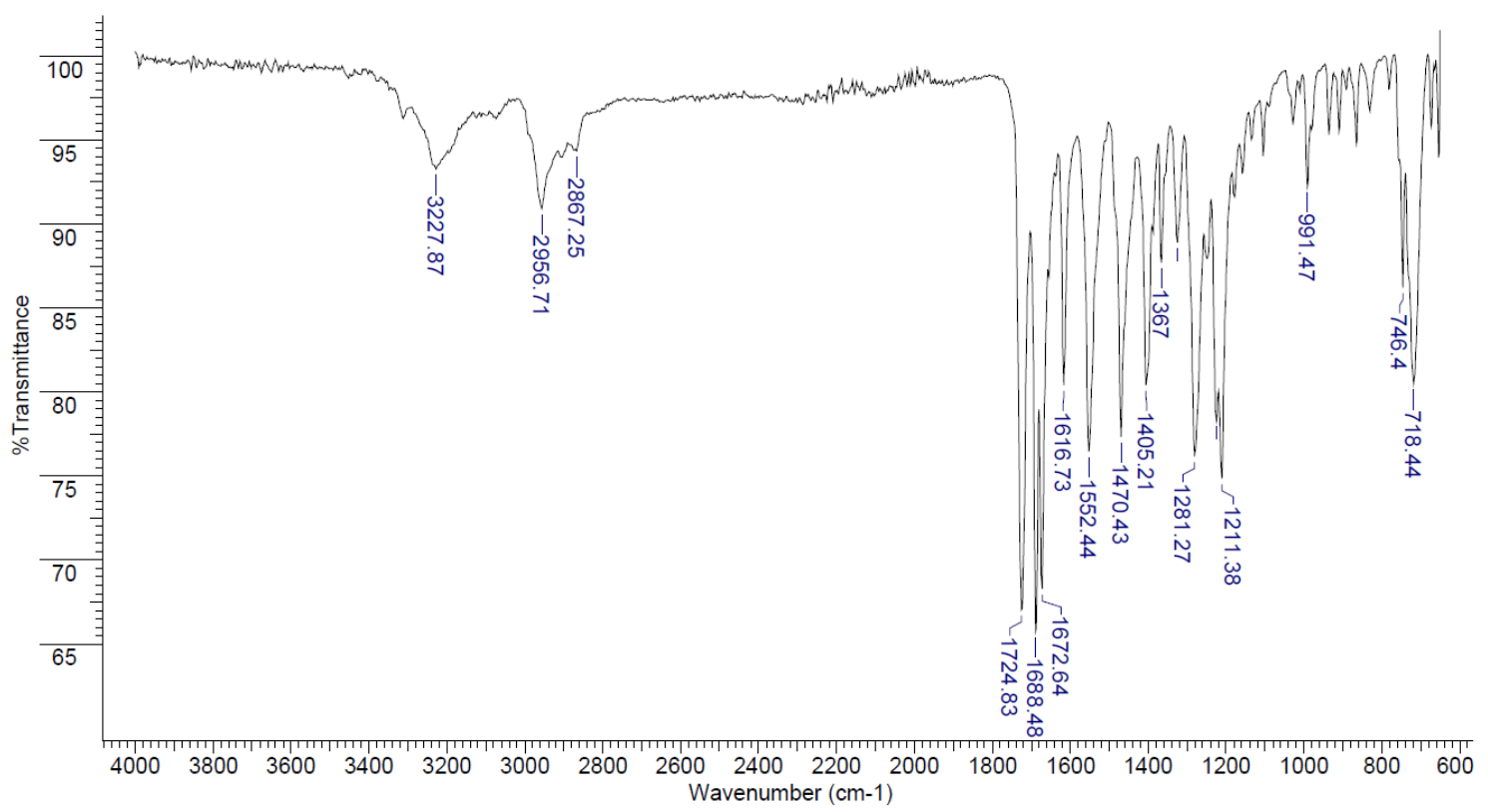

$5 a d b$

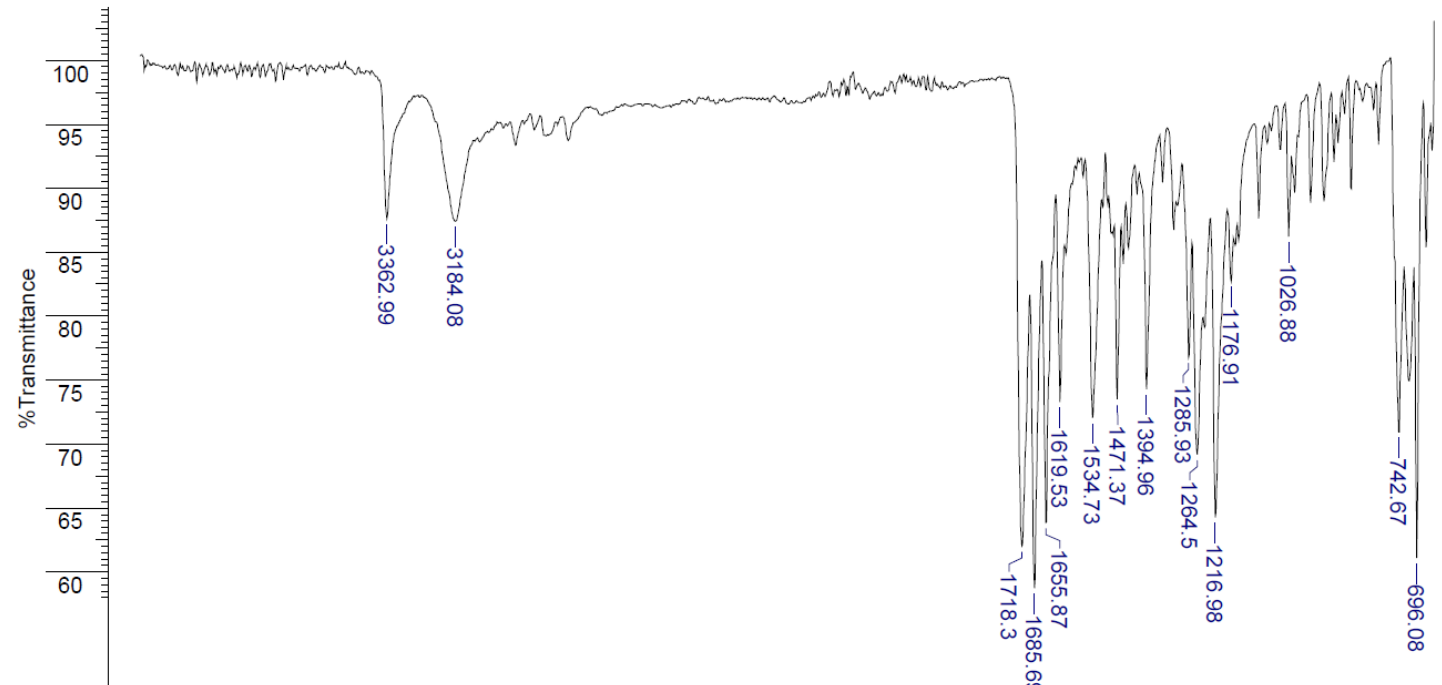

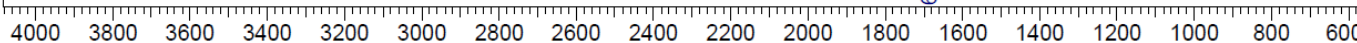
Wavenumber (cm-1) 
$5 b c b$

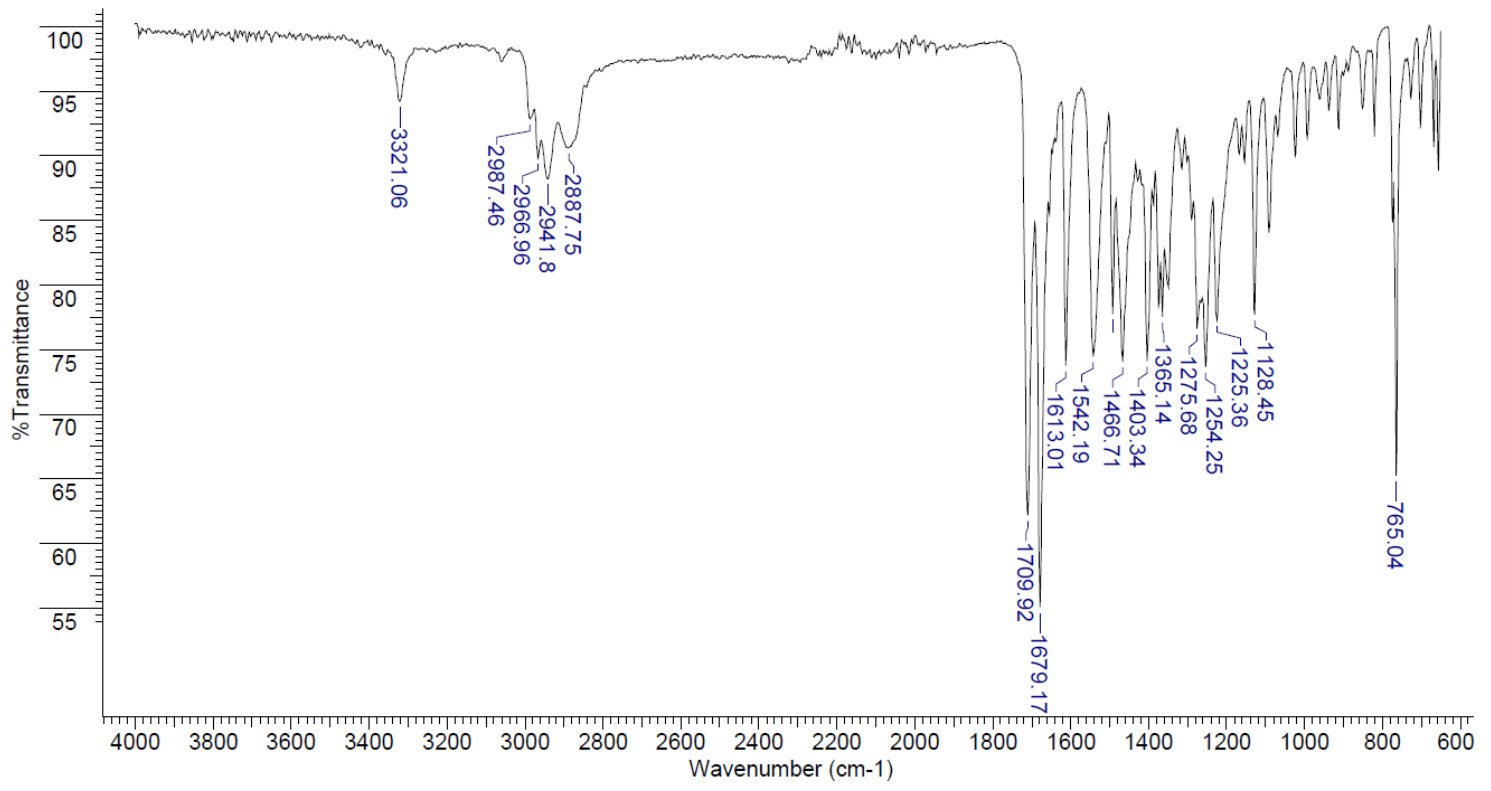

\section{$5 c d b$}

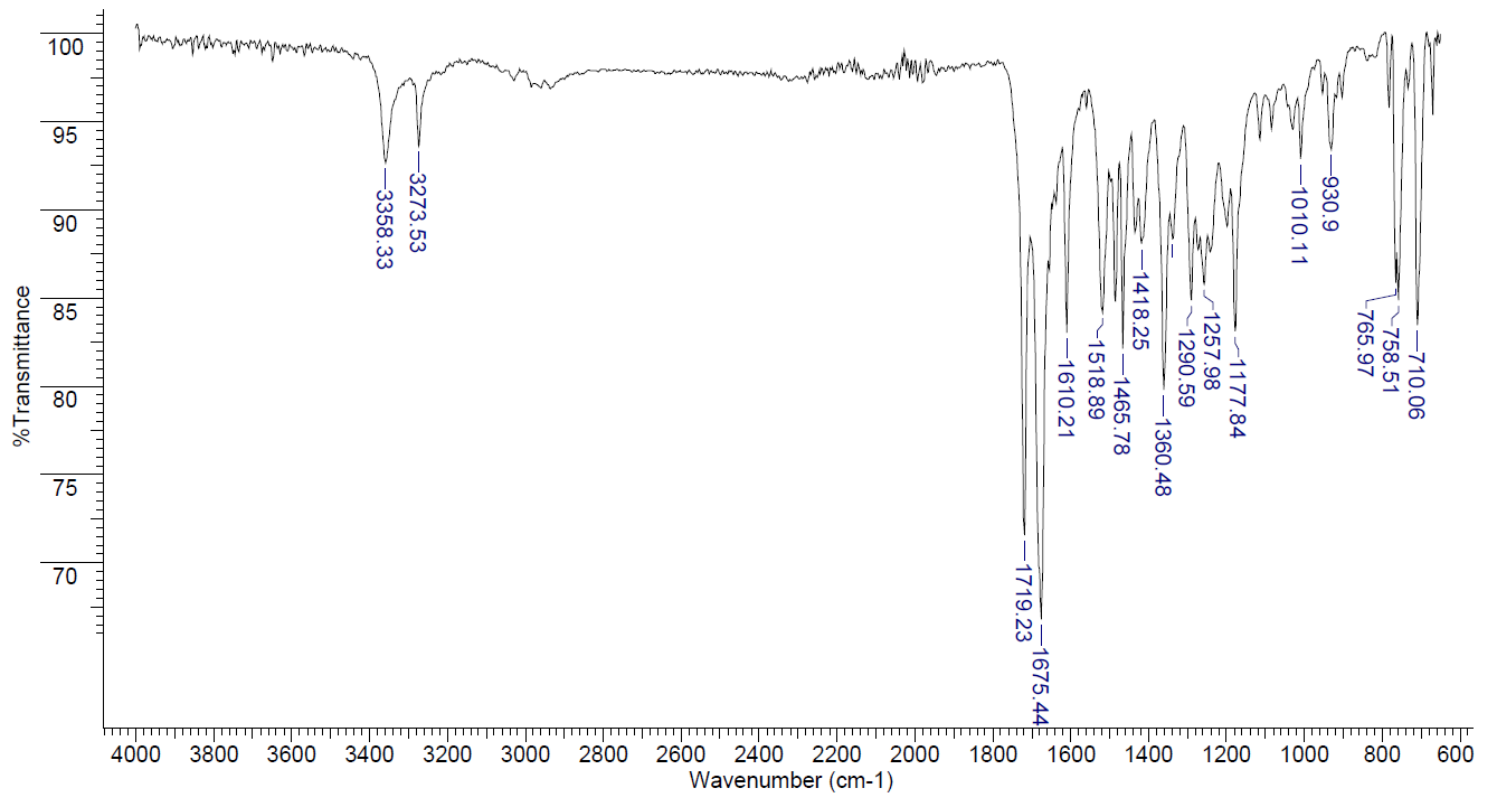




\section{Swiss $A D M E \circledast$ evaluation reports}

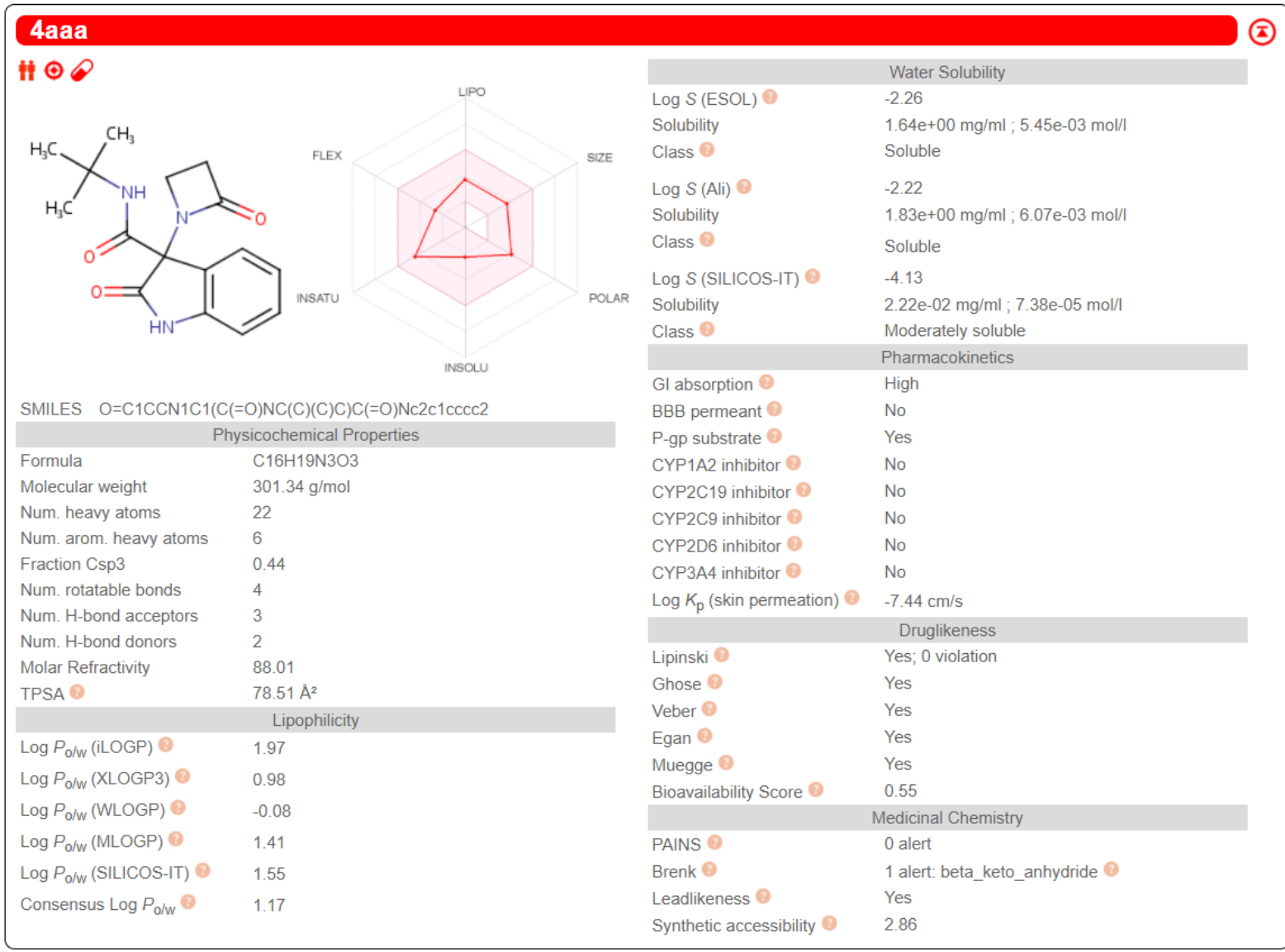

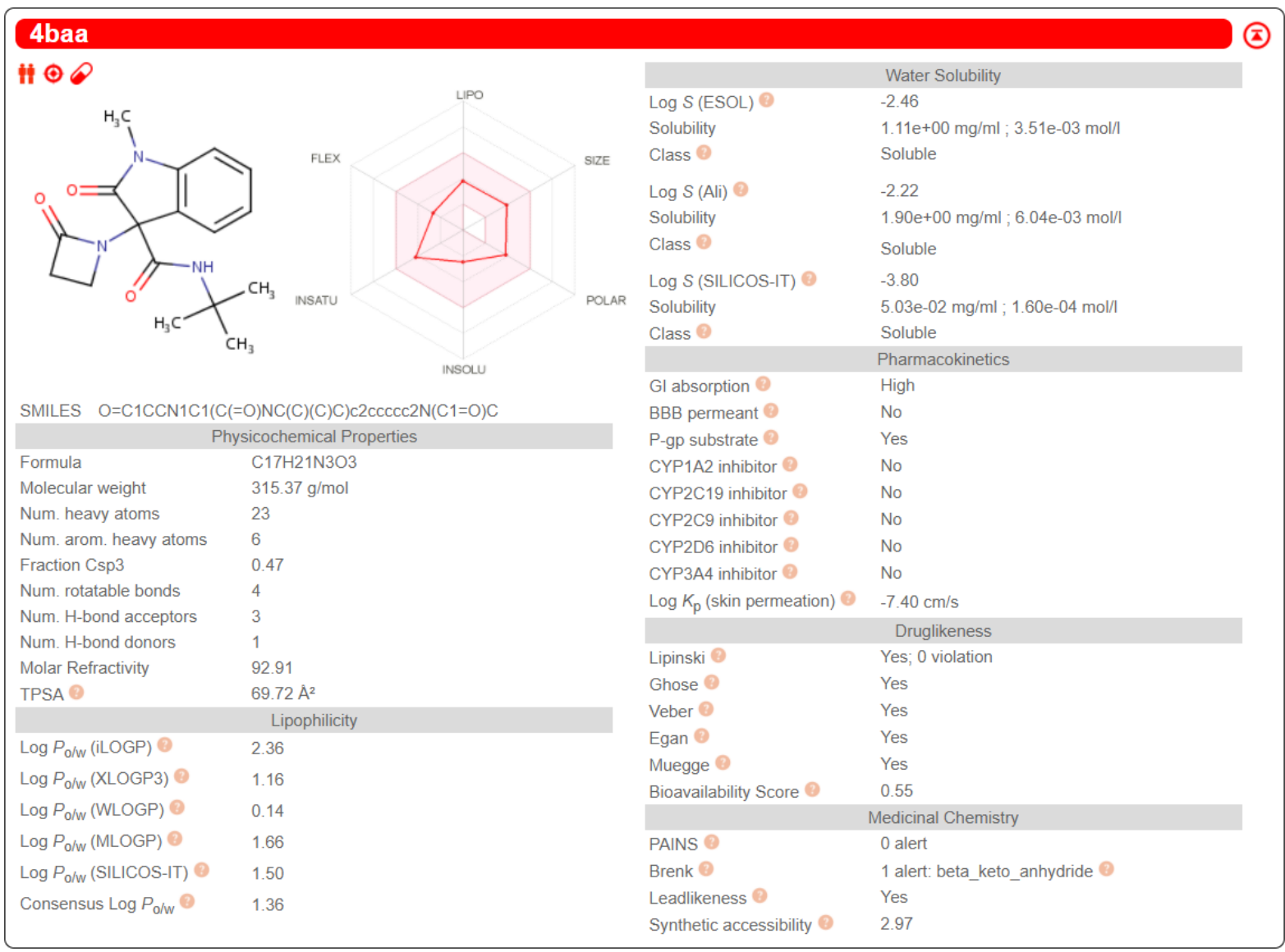




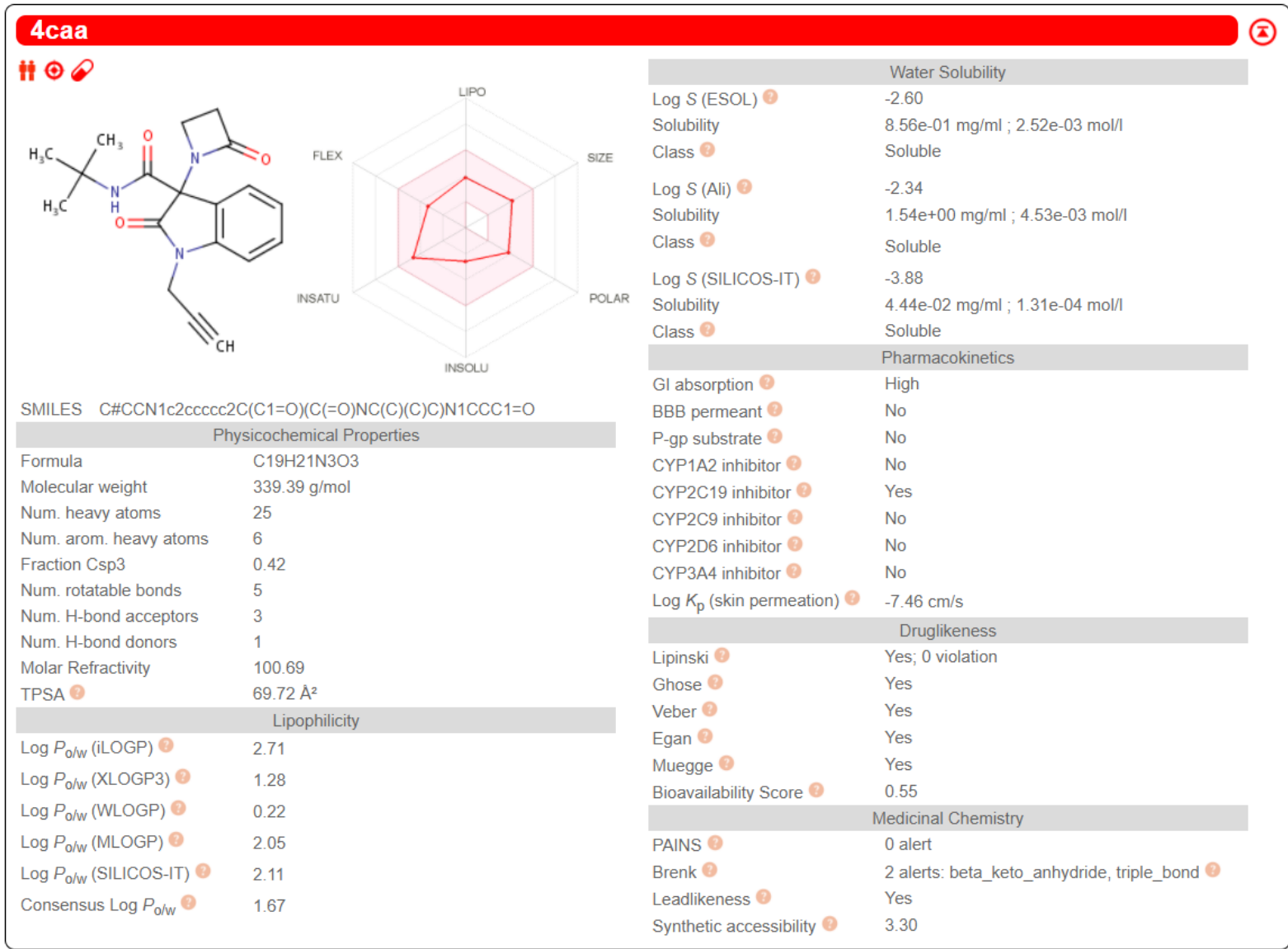

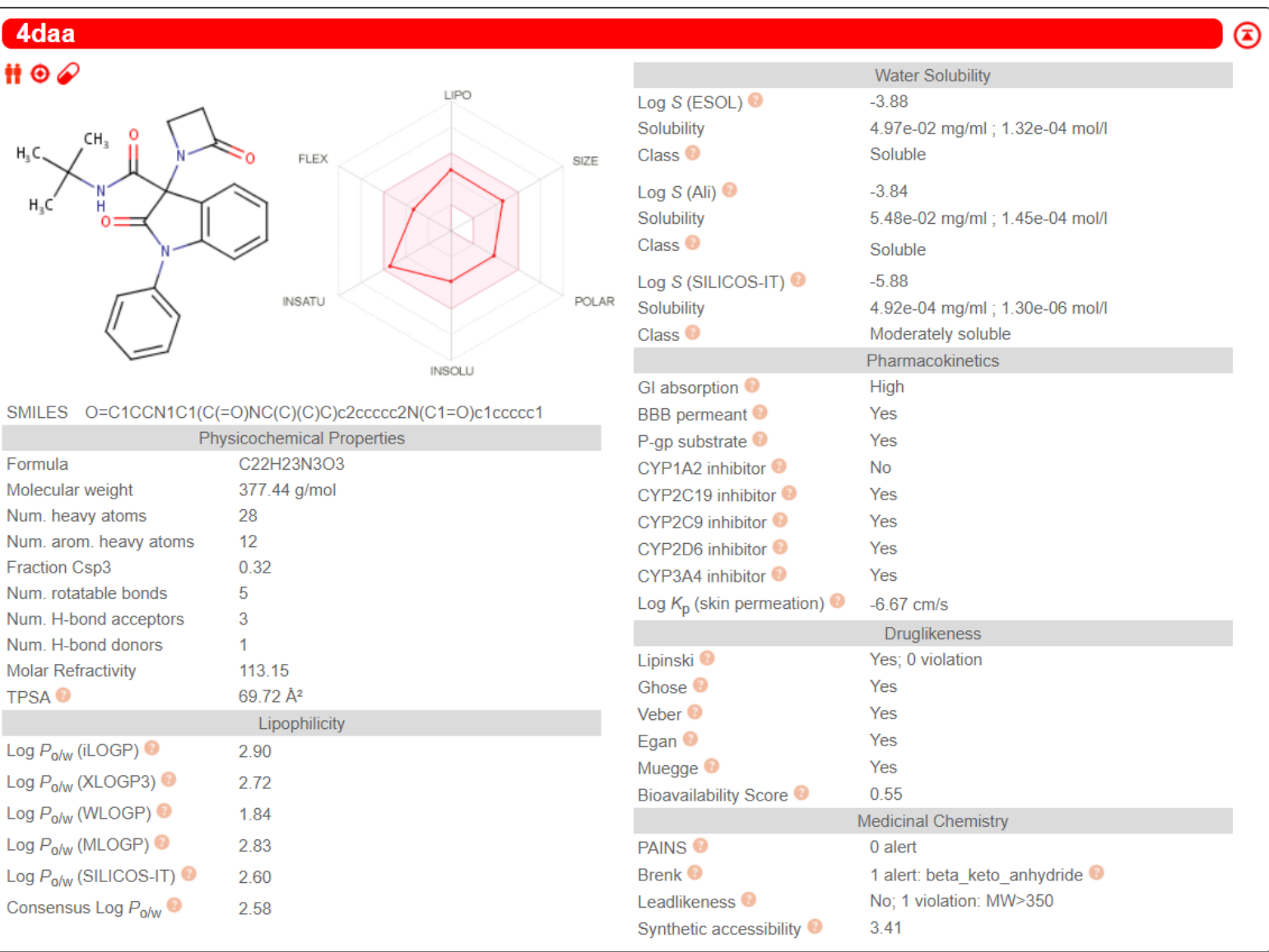




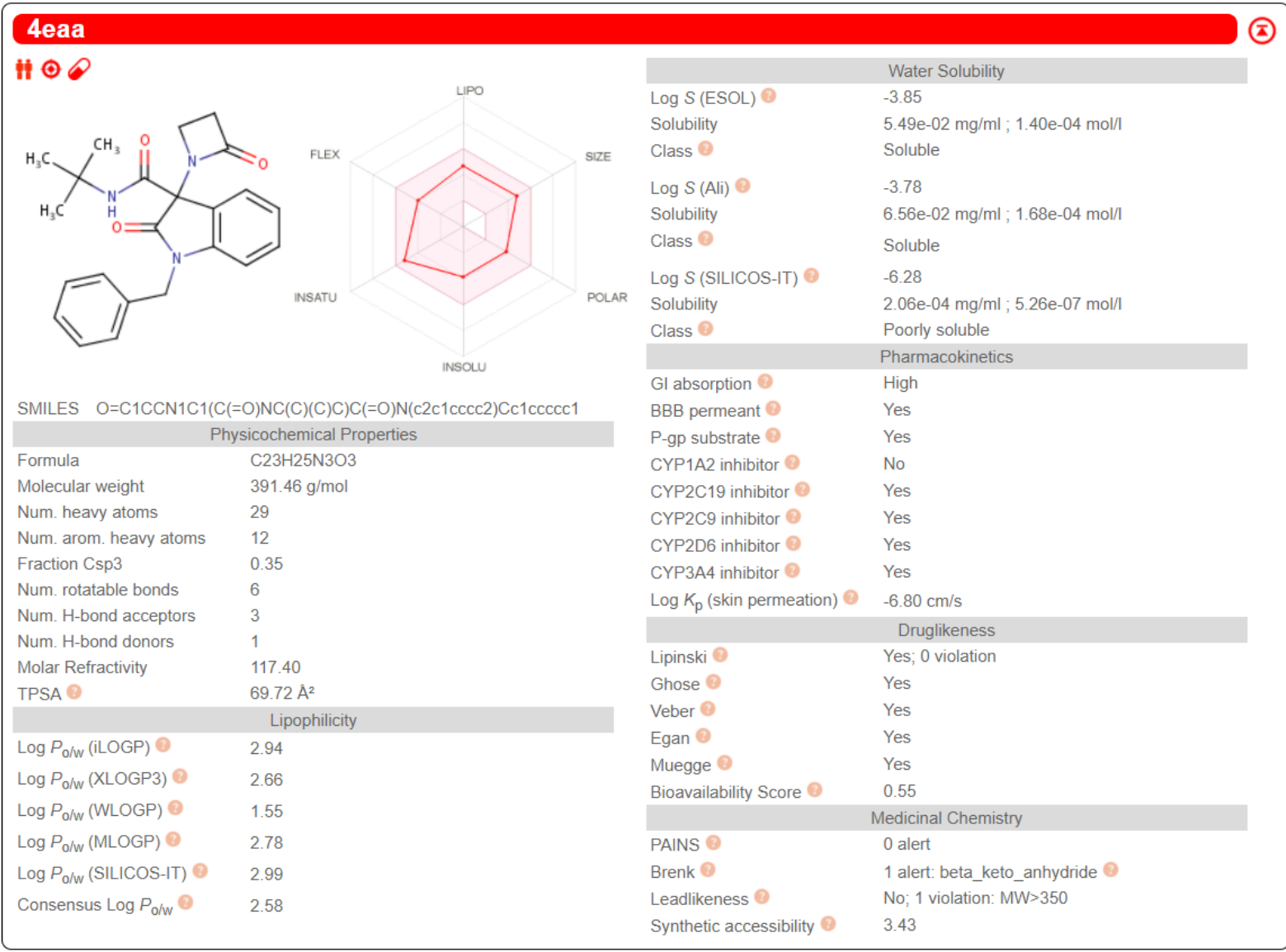

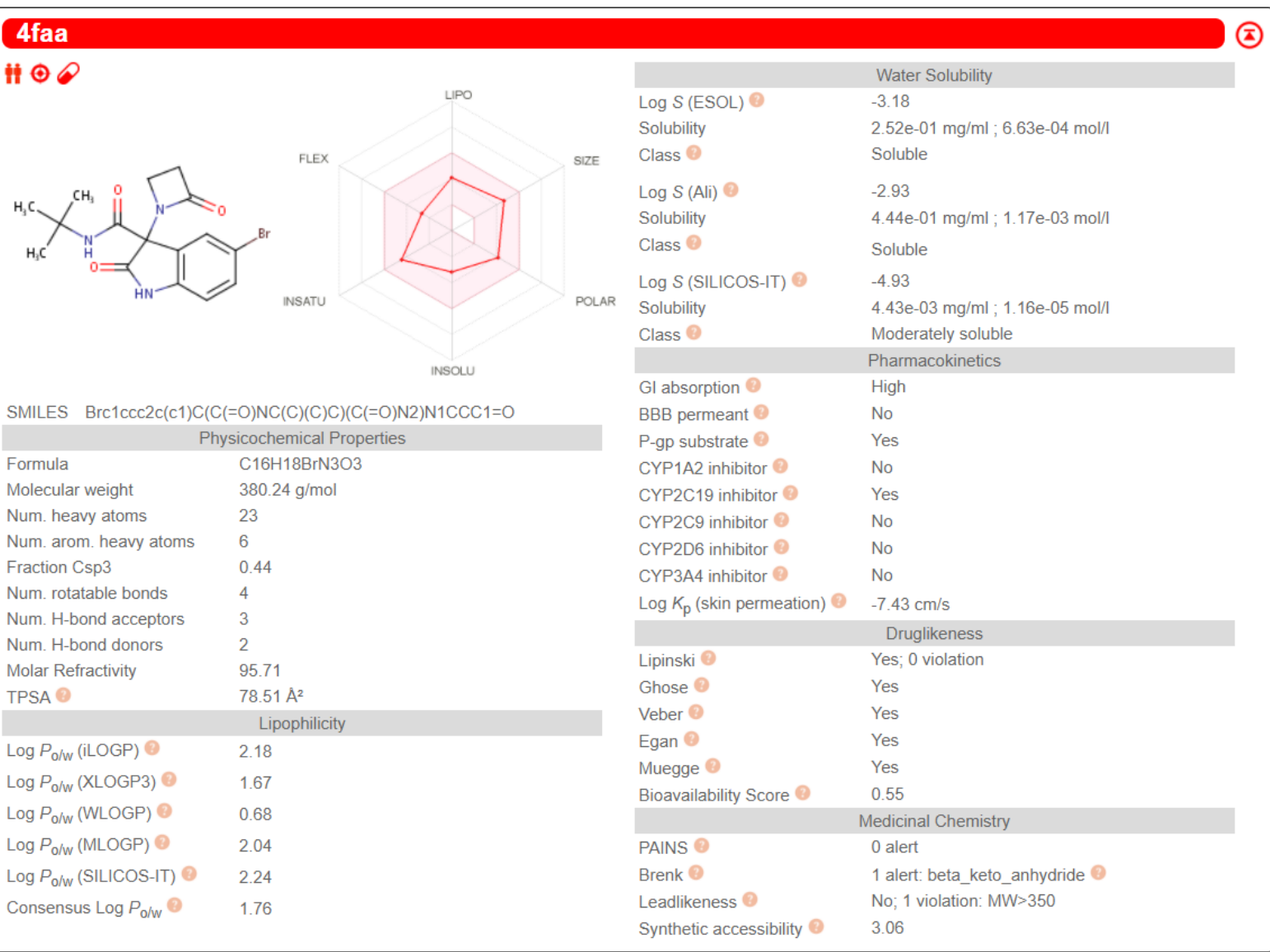




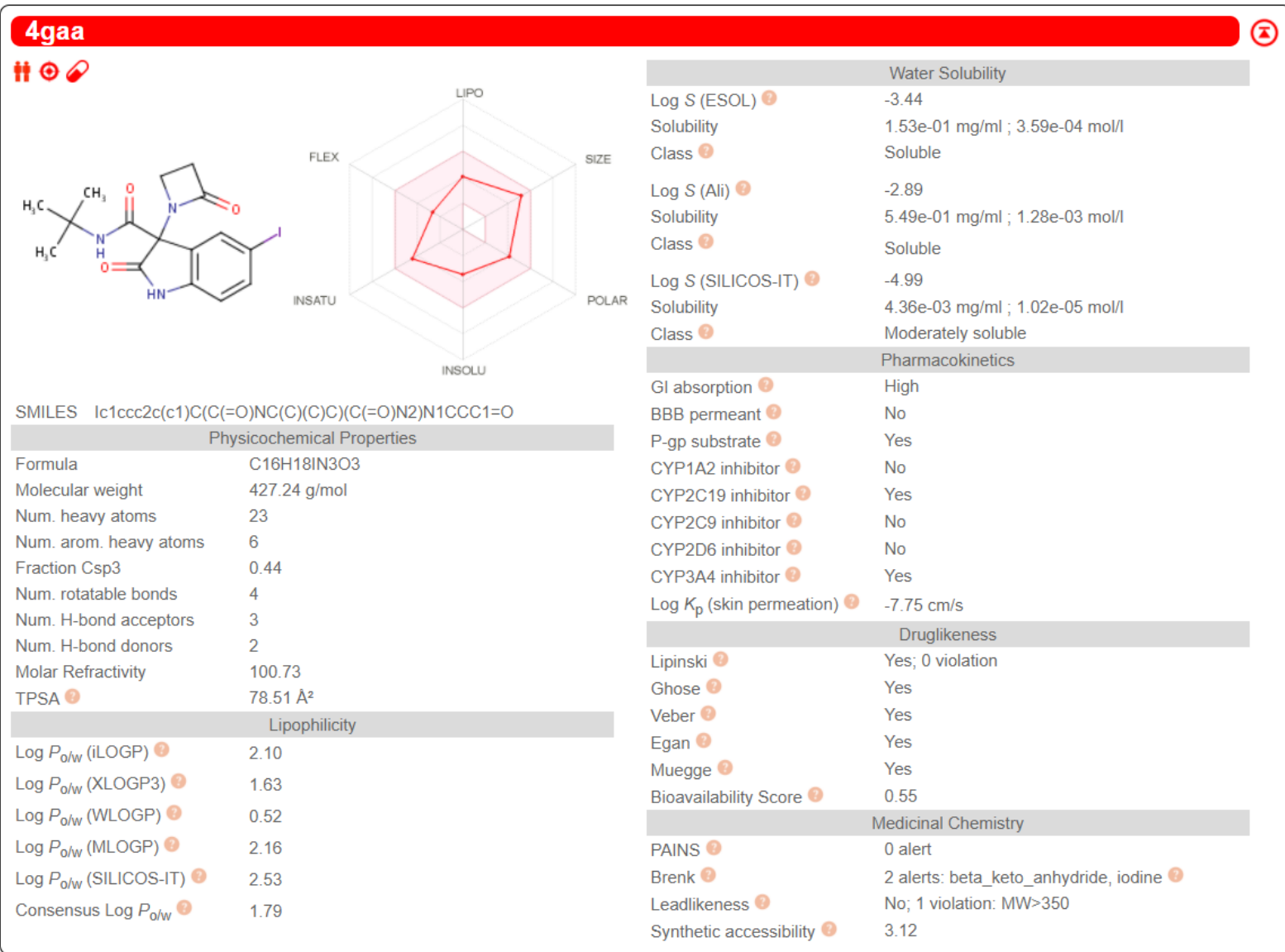

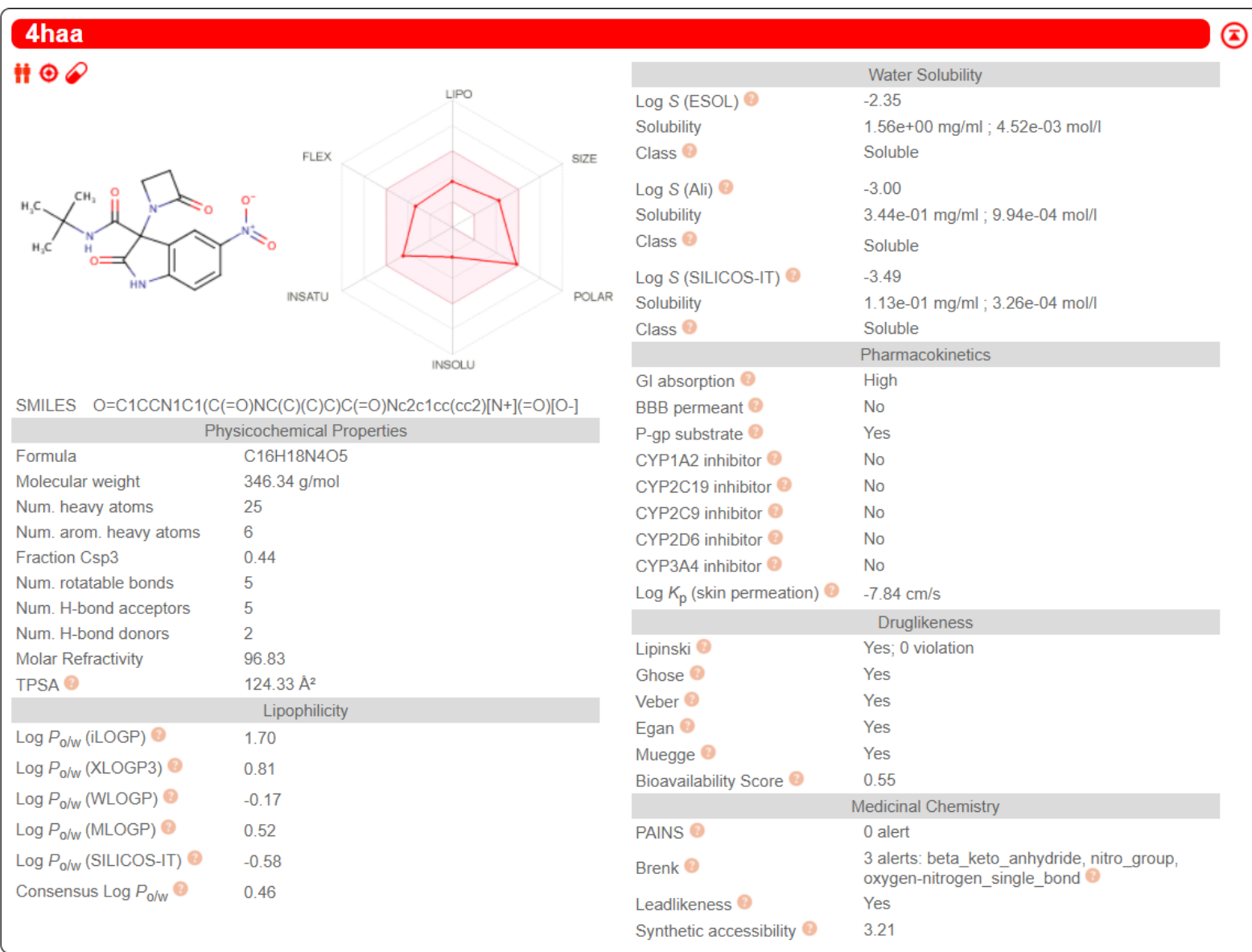




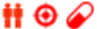<smiles>Cc1cc(C)c2c(c1)C(C(=O)NC(C)(C)C)(N1CCC1=O)C(=O)N2</smiles>

FLEX

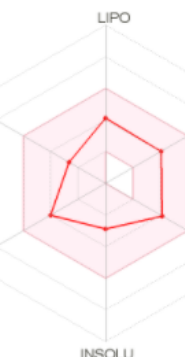

NSOLU
SMILES $\quad C c 1 c c(C) c 2 c(c 1) C(C(=O) N 2)(C(=O) N C(C)(C) C) N 1 C C C 1=0$ Physicochemical Properties

Formula

Molecular weight $\quad 329.39 \mathrm{~g} / \mathrm{mol}$

Num. heavy atoms $\quad 24$

Num. arom. heavy atoms 6

Fraction Csp3 $\quad 0.50$

Num. rotatable bonds $\quad 4$

Num. H-bond acceptors 3

Num. H-bond donors 2

Molar Refractivity $\quad 97.94$

TPSA $278.51 \AA^{2}$

\begin{tabular}{|c|c|}
\hline & Lipophilicity \\
\hline $\log P_{o / w}$ (iLOGP) $P$ & 2.35 \\
\hline $\log P_{\mathrm{o} / \mathrm{w}}(\mathrm{XLOGP} 3)$ & 1.71 \\
\hline $\log P_{0 / w}($ WLOGP) 0 & 0.54 \\
\hline $\log P_{\mathrm{o} / \mathrm{w}}(\mathrm{MLOGP})$ & 1.89 \\
\hline $\log P_{\mathrm{o} / \mathrm{w}}(\mathrm{SILICOS}-\mathrm{IT})(2)$ & 2.59 \\
\hline Consensus Log $P_{\mathrm{o} / \mathrm{w}}$ & 1.82 \\
\hline
\end{tabular}

SIZE

POLAR

Water Solubility

$-2.88$

Solubility $\quad 4.34 \mathrm{e}-01 \mathrm{mg} / \mathrm{ml} ; 1.32 \mathrm{e}-03 \mathrm{~mol} / \mathrm{l}$

Class 18 Soluble

Log S (Ali) $9-2.97$

Solubility $\quad 3.49 \mathrm{e}-01 \mathrm{mg} / \mathrm{ml} ; 1.06 \mathrm{e}-03 \mathrm{~mol} / \mathrm{l}$

Class 0 Soluble

Log S (SILICOS-IT) 0

Solubility

Moderately soluble

Pharmacokinetics

High

BBB permeant $(2) \quad$ No

P-gp substrate $P$ Yes

CYP1A2 inhibitor $2 \quad$ No

CYP2C19 inhibitor 9 Yes

CYP2C9 inhibitor 2 No

CYP2D6 inhibitor 9 No

CYP3A4 inhibitor 0 No

$\log K_{\mathrm{p}}$ (skin permeation) $-7.10 \mathrm{~cm} / \mathrm{s}$

\begin{tabular}{ll|} 
& Druglikeness \\
\hline Lipinski $\odot$ & Yes; 0 violation \\
Ghose $\odot$ & Yes \\
Veber $\odot$ & Yes \\
Egan $\odot$ & Yes \\
Muegge $\odot$ & Yes \\
Bioavailability Score $\odot$ & 0.55 \\
\hline PAINS $\odot$ & Medicinal Chemistry \\
Brenk $\odot$ & 0 alert \\
Leadlikeness $\odot$ & 1 alert: beta_keto_anhydride $\odot$ \\
Synthetic accessibility $\odot$ & Yes \\
\hline
\end{tabular}

\section{4jaa}

\section{$H \odot \odot$}

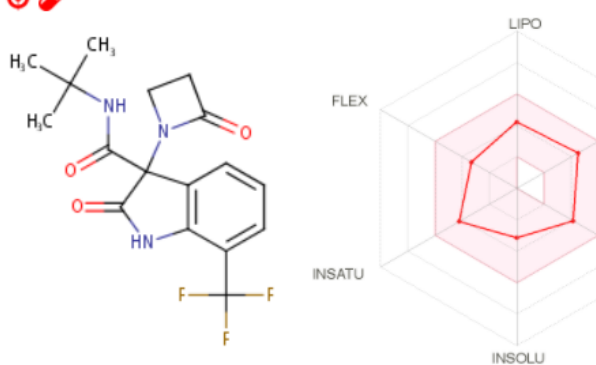

SMILES $O=C 1 C C N 1 C 1(C(=O) N C(C)(C) C) C(=O) N c 2 c 1 \operatorname{cccc} 2 C(F)(F) F$ Physicochemical Properties

Formula

$\mathrm{C} 17 \mathrm{H} 18 \mathrm{~F} 3 \mathrm{~N} 3 \mathrm{O} 3$

Molecular weight $\quad 369.34 \mathrm{~g} / \mathrm{mol}$

Num. heavy atoms $\quad 26$

Num. arom. heavy atoms 6

$\begin{array}{ll}\text { Fraction Csp3 } & 0.47\end{array}$

Num. rotatable bonds $\quad 5$

Num. H-bond acceptors $\quad 6$

Num. H-bond donors 2

Molar Refractivity $\quad 93.01$

TPSA $\odot 78.51 \AA^{2}$

\begin{tabular}{ll} 
& Lipophilicity \\
\hline $\log P_{\mathrm{o} / \mathrm{w}}($ (ILOGP) & 2.70 \\
$\log P_{\mathrm{o} / \mathrm{w}}($ XLOGP 3$) \odot$ & 1.86 \\
$\log P_{\mathrm{o} / \mathrm{w}}($ (WLOGP) $\odot$ & 2.09 \\
$\log P_{\mathrm{o} / \mathrm{w}}(\mathrm{MLOGP}) \odot$ & 2.28 \\
$\log P_{\mathrm{o} / \mathrm{w}}(\mathrm{SILICOS}-\mathrm{IT}) \odot$ & 2.65 \\
Consensus Log $P_{\mathrm{o} / \mathrm{w}} \odot$ & 2.32
\end{tabular}

$\begin{array}{lll} & \text { Log } S(E S O L) ~ & -3.14 \\ \text { Solubility } & 2.66 \mathrm{e}-01 \mathrm{mg} / \mathrm{ml} ; 7.20 \mathrm{e}-04 \mathrm{~mol} / \mathrm{l} \\ \text { SIZE } & \text { Class } 0 & \text { Soluble }\end{array}$

(2)

$\log S$ (Ali) $2+3.13$

Solubility $\quad 2.74 \mathrm{e}-01 \mathrm{mg} / \mathrm{ml} ; 7.41 \mathrm{e}-04 \mathrm{~mol} / \mathrm{l}$

Class 9 Soluble

$\log S$ (SILICOS-IT) 0

POLAR Solubility $3.89 \mathrm{e}-03 \mathrm{mg} / \mathrm{ml} ; 1.05 \mathrm{e}-05 \mathrm{~mol} / \mathrm{l}$

Class (2) Moderately soluble

Pharmacokinetics

Gl absorption 9 High

BBB permeant 2 No

P-gp substrate $(2) \quad$ Yes

CYP1A2 inhibitor 2 No

CYP2C19 inhibitor 2 Yes

CYP2C9 inhibitor 2 No

CYP2D6 inhibitor $12 \quad$ No

CYP3A4 inhibitor 9

$\log K_{\mathrm{p}}$ (skin permeation) $3-7.23 \mathrm{~cm} / \mathrm{s}$

\begin{tabular}{|c|c|}
\hline \multicolumn{2}{|r|}{ Druglikeness } \\
\hline Lipinski 2 & Yes; 0 violation \\
\hline Ghose $(8$ & Yes \\
\hline Veber $\odot$ & Yes \\
\hline Egan 0 & Yes \\
\hline Muegge 0 & Yes \\
\hline Bioavailability Score 2 & 0.55 \\
\hline \multicolumn{2}{|r|}{ Medicinal Chemistry } \\
\hline PAINS 2 & 0 alert \\
\hline Brenk $\odot$ & 1 alert: beta_keto_anhydride 0 \\
\hline Leadlikeness 2 & No; 1 violation: $M W>350$ \\
\hline Synthetic accessibility (2) & 3.27 \\
\hline
\end{tabular}




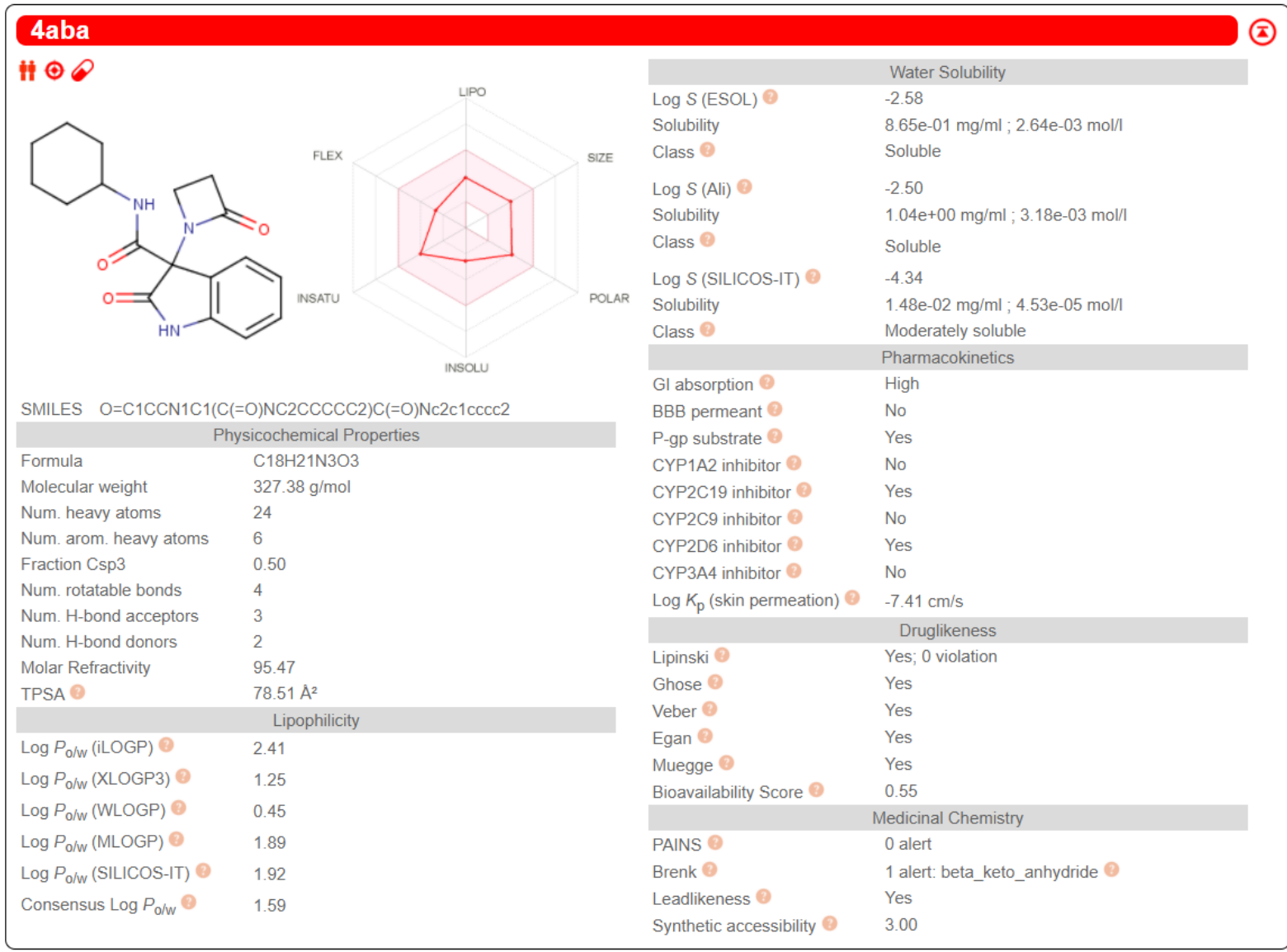

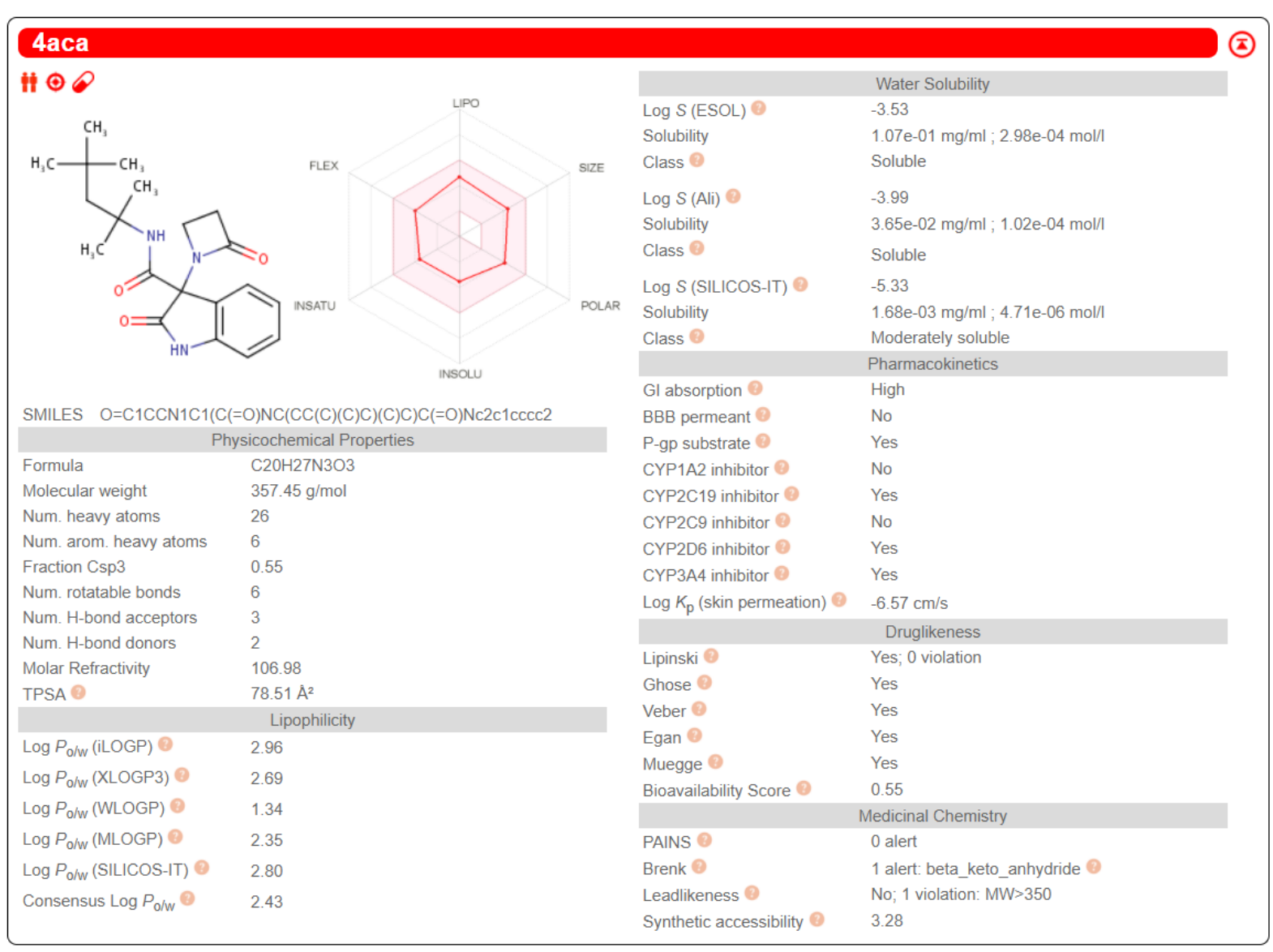




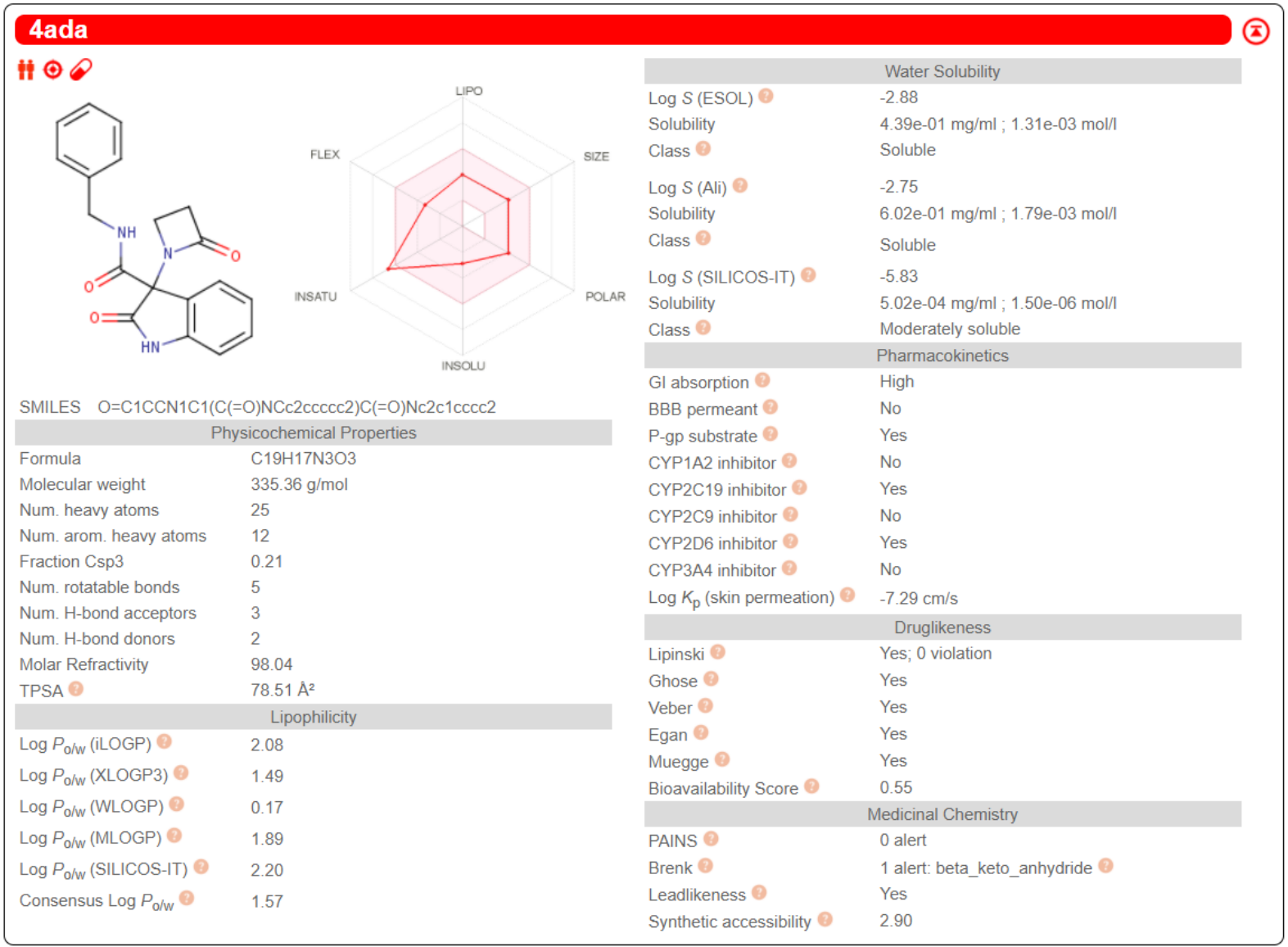

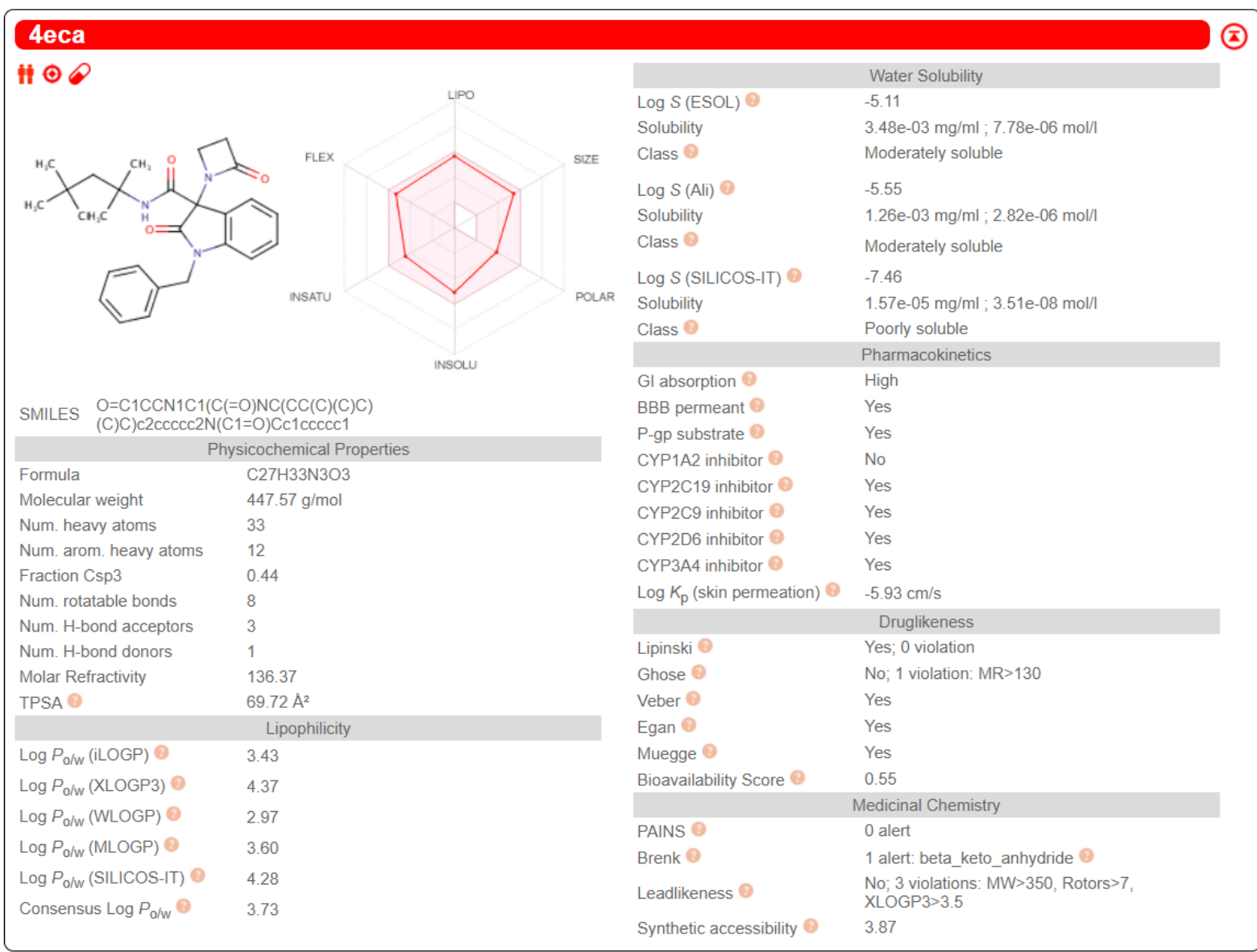


<smiles>C#CCN1C(=O)C(C(=O)NCc2ccccc2)(N2CCC2=O)c2ccccc21</smiles>

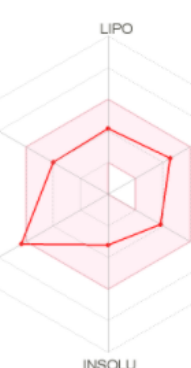

SMILES C\#CCN1c2ccccc2C $(C 1=O)(C(=O) N C c 1 c c c c c 1) N 1 C C C 1=0$ Physicochemical Properties

$\begin{array}{ll}\text { Formula } & \mathrm{C} 22 \mathrm{H} 19 \mathrm{~N} 3 \mathrm{O} 3 \\ \text { Molecular weight } & 373.40 \mathrm{~g} / \mathrm{mol} \\ \text { Num. heavy atoms } & 28 \\ \text { Num. arom. heavy atoms } & 12 \\ \text { Fraction Csp3 } & 0.23 \\ \text { Num. rotatable bonds } & 6 \\ \text { Num. H-bond acceptors } & 3 \\ \text { Num. H-bond donors } & 1 \\ \text { Molar Refractivity } & 110.72 \\ \text { TPSA } 0 & 69.72 \AA^{2}\end{array}$

Lipophilicity

$\log P_{\mathrm{o} / \mathrm{w}}$ (iLOGP) 2

$\log P_{\mathrm{o} / \mathrm{w}}(\mathrm{XLOGP} 3) \odot 1.79$

$\log P_{\mathrm{o} / \mathrm{w}}$ (WLOGP) 00.47

$\log P_{\mathrm{o} / \mathrm{w}}$ (MLOGP) $2 \quad 2.49$

$\log P_{\mathrm{o} / \mathrm{w}}$ (SILICOS-IT) 02.75

Consensus Log $P_{\mathrm{o} / \mathrm{w}} \odot 2.03$
Water Solubility

$-3.20$

$2.33 \mathrm{e}-01 \mathrm{mg} / \mathrm{ml} ; 6.25 \mathrm{e}-04 \mathrm{~mol} / \mathrm{l}$

SIZE

Solubility $2.33 \mathrm{e}-01$

Log $S$ (Ali) $12 \quad-2.87$

Solubility $\quad 5.00 \mathrm{e}-01 \mathrm{mg} / \mathrm{ml} ; 1.34 \mathrm{e}-03 \mathrm{~mol} / \mathrm{l}$

Class 8 Soluble

Log S (SILICOS-IT) P -5.57

POLAR Solubility $1.00 \mathrm{e}-03 \mathrm{mg} / \mathrm{ml} ; 2.69 \mathrm{e}-06 \mathrm{~mol} / \mathrm{l}$

Class $1 \quad$ Moderately soluble

\begin{tabular}{|c|c|}
\hline \multicolumn{2}{|r|}{ Pharmacokinetics } \\
\hline Gl absorption 3 & High \\
\hline BBB permeant 9 & No \\
\hline P-gp substrate 2 & Yes \\
\hline CYP1A2 inhibitor 9 & No \\
\hline CYP2C19 inhibitor 9 & Yes \\
\hline CYP2C9 inhibitor 8 & Yes \\
\hline CYP2D6 inhibitor 3 & Yes \\
\hline CYP3A4 inhibitor 0 & Yes \\
\hline $\log K_{p}$ (skin permeation) 3 & $-7.31 \mathrm{~cm} / \mathrm{s}$ \\
\hline \multicolumn{2}{|r|}{ Druglikeness } \\
\hline Lipinski 0 & Yes; 0 violation \\
\hline Ghose 9 & Yes \\
\hline Veber 9 & Yes \\
\hline Egan 0 & Yes \\
\hline Muegge $\odot$ & Yes \\
\hline Bioavailability Score 2 & 0.55 \\
\hline \multicolumn{2}{|r|}{ Medicinal Chemistry } \\
\hline PAINS 0 & 0 alert \\
\hline Brenk 2 & 2 alerts: beta_keto_anhydride, triple_bond $(2$ \\
\hline Leadlikeness 3 & No; 1 violation: $M W>350$ \\
\hline Synthetic accessibility 2 & 3.35 \\
\hline
\end{tabular}

\section{$4 \mathrm{bba}$}

if $\odot \bigcirc$
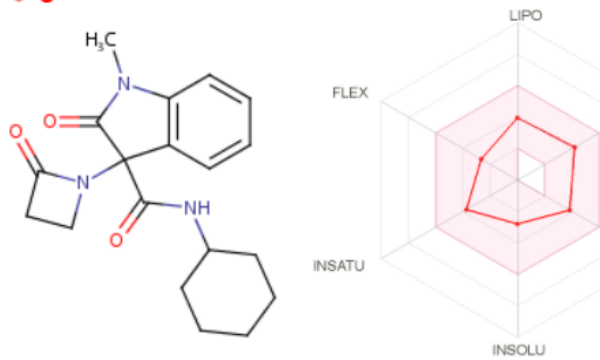

SMILES O=C1CCN1C1 $(\mathrm{C}(=\mathrm{O}) \mathrm{NC} 2 \mathrm{CCCCC} 2) \operatorname{cocccc} 2 \mathrm{~N}(\mathrm{C} 1=\mathrm{O}) \mathrm{C}$

$\begin{array}{ll} & \text { Physicochemical Pro } \\ \text { Formula } & \mathrm{C} 19 \mathrm{H} 23 \mathrm{~N} 3 \mathrm{O} 3 \\ \text { Molecular weight } & 341.40 \mathrm{~g} / \mathrm{mol} \\ \text { Num. heavy atoms } & 25 \\ \text { Num. arom. heavy atoms } & 6 \\ \text { Fraction Csp3 } & 0.53 \\ \text { Num. rotatable bonds } & 4 \\ \text { Num. H-bond acceptors } & 3 \\ \text { Num. H-bond donors } & 1 \\ \text { Molar Refractivity } & 100.37 \\ \text { TPSA P } & 69.72 \AA^{2}\end{array}$

$\begin{aligned} 69.72 \mathrm{~A}^{2} & \text { Lipophilicity }\end{aligned}$

$\log P_{\mathrm{o} / \mathrm{w}}$ (iLOGP) 2.50

$\log P_{\text {o/w }}($ XLOGP3) 1.44

$\log P_{\mathrm{o} / \mathrm{w}}($ WLOGP) 00.67

$\log P_{\mathrm{o} / \mathrm{W}}(\mathrm{MLOGP}) \odot \quad 2.13$

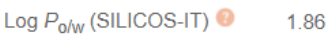

Consensus $\log P_{\mathrm{o} / \mathrm{w}} \odot \quad 1.72$

\begin{tabular}{lll} 
& \multicolumn{2}{c}{ Water Solubility } \\
& Log $S(E S O L) ~$ & -2.78 \\
Solubility & $5.70 \mathrm{e}-01 \mathrm{mg} / \mathrm{ml} ; 1.67 \mathrm{e}-03 \mathrm{~mol} / \mathrm{l}$ \\
SIzE & Class 0 & Soluble
\end{tabular}

Log $S$ (Ali) $\odot-2.51$

Solubility $\quad 1.06 \mathrm{e}+00 \mathrm{mg} / \mathrm{ml} ; 3.09 \mathrm{e}-03 \mathrm{~mol} / \mathrm{l}$
Soluble

$\log S$ (SILICOS-IT) $10 \quad-4.01$

POLAR Solubility $3.35 \mathrm{e}-02 \mathrm{mg} / \mathrm{ml} ; 9.82 \mathrm{e}-05 \mathrm{~mol} / \mathrm{l}$

Class (2) Moderately soluble

Pharmacokinetics

Gl absorption (2) High

BBB permeant $(2) \quad$ No

P-gp substrate $2 \quad$ Yes

CYP1A2 inhibitor 9 No

CYP2C19 inhibitor 9 Yes

CYP2C9 inhibitor 10

CYP2D6 inhibitor 0 Yes

CYP3A4 inhibitor (2) No

Log $K_{\mathrm{p}}$ (skin permeation) $-7.36 \mathrm{~cm} / \mathrm{s}$

\begin{tabular}{|c|c|}
\hline \multicolumn{2}{|r|}{ Druglikeness } \\
\hline Lipinski 3 & Yes; 0 violation \\
\hline Ghose 2 & Yes \\
\hline Veber 2 & Yes \\
\hline Egan 2 & Yes \\
\hline Muegge 2 & Yes \\
\hline Bioavailability Score & 0.55 \\
\hline \multicolumn{2}{|r|}{ Medicinal Chemistry } \\
\hline PAINS 0 & 0 alert \\
\hline Brenk 2 & 1 alert: beta_keto_anhydride $(2)$ \\
\hline Leadlikeness 0 & Yes \\
\hline Synthetic accessibility & 3.11 \\
\hline
\end{tabular}




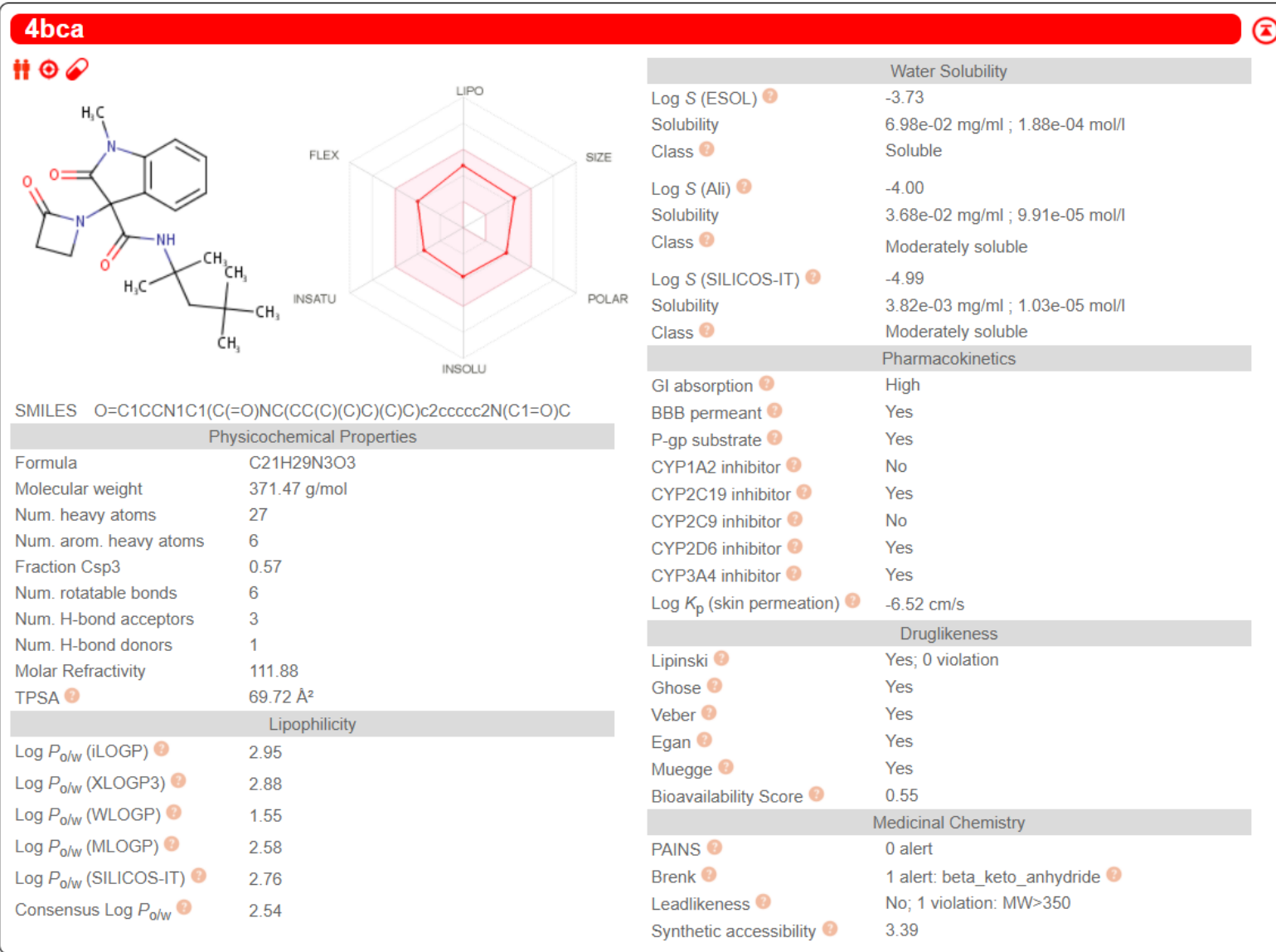




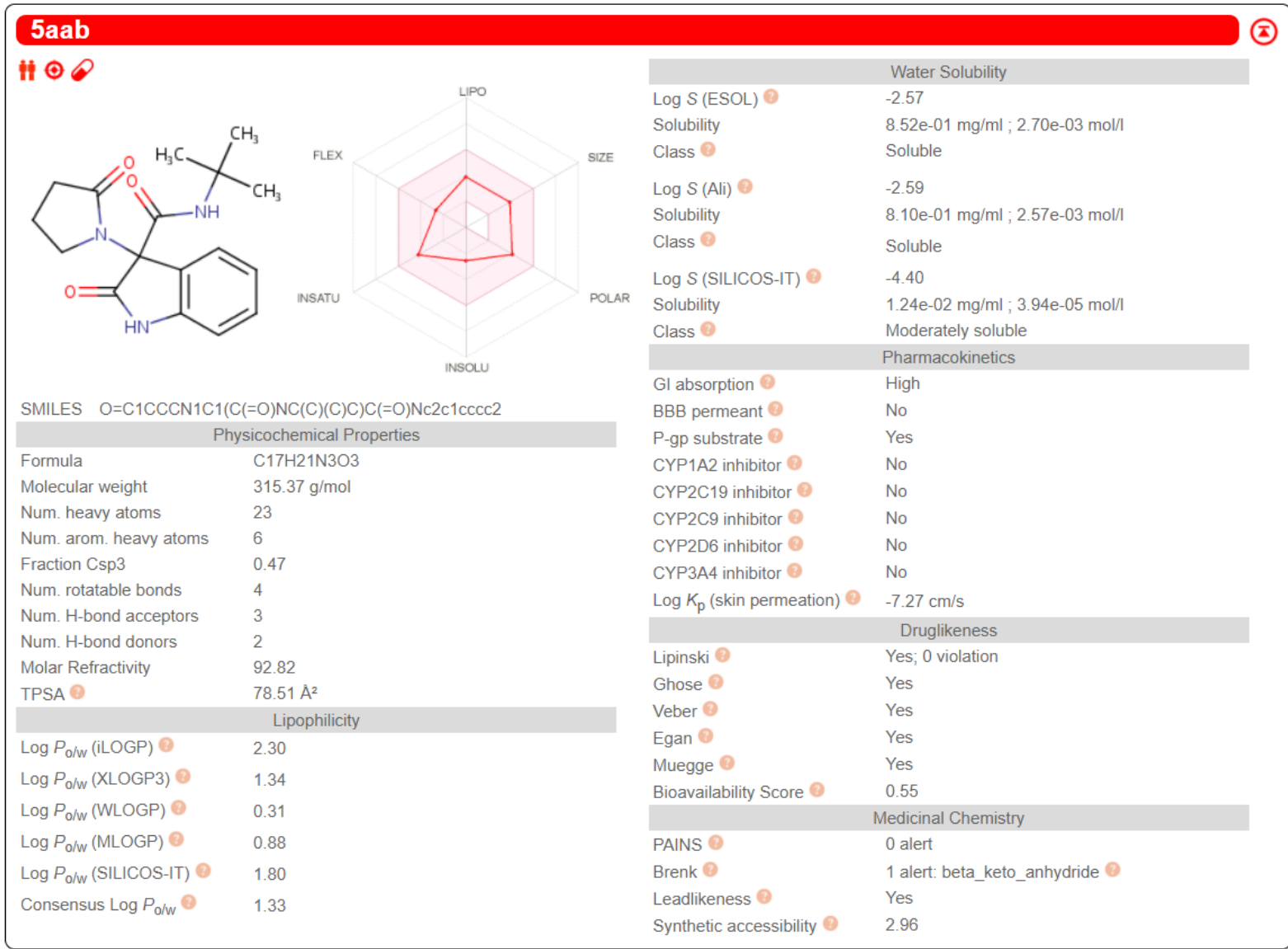

\begin{tabular}{|c|c|c|c|c|}
\hline 5 bab & & & & \\
\hline$\| \odot 0$ & & & & Water Solubility \\
\hline & & & $\log S(E S O L) \odot$ & -2.76 \\
\hline & & & Solubility & $5.71 \mathrm{e}-01 \mathrm{mg} / \mathrm{ml} ; 1.73 \mathrm{e}-03 \mathrm{~mol} / \mathrm{l}$ \\
\hline & FLEX & SIZE & Class 8 & Soluble \\
\hline & & & $\log S$ (Ali) 1 & -2.59 \\
\hline & & & Solubility & $8.42 \mathrm{e}-01 \mathrm{mg} / \mathrm{ml} ; 2.55 \mathrm{e}-03 \mathrm{~mol} / 1$ \\
\hline & & & Class 3 & Soluble \\
\hline & & POAP & $\log S$ (SILICOS-IT) $\odot$ & -4.07 \\
\hline & & ren & Solubility & $\begin{array}{l}2.82 \mathrm{e}-02 \mathrm{mg} / \mathrm{ml} ; 8.55 \mathrm{e}-05 \mathrm{~mol} / \mathrm{l} \\
\text { Moderately soluble }\end{array}$ \\
\hline & (1) & & & Pharmacokinetics \\
\hline & INSOL & & Gl absorption $\odot$ & High \\
\hline SMILES $O=C 1 C C C N 1 C$ & $=0) N C(C)(C) C) \operatorname{cocccc} 2 N(C 1=O) C$ & & BBB permeant $\odot$ & No \\
\hline & sicochemical Properties & & P-gp substrate 8 & Yes \\
\hline Formula & $\mathrm{C} 18 \mathrm{H} 23 \mathrm{~N} 303$ & & CYP1A2 inhibitor ${ }^{\circ}$ & No \\
\hline Molecular weight & $329.39 \mathrm{~g} / \mathrm{mol}$ & & CYP2C19 inhibitor 0 & Yes \\
\hline Num. heavy atoms & 24 & & CYP2C9 inhibitor 9 & No \\
\hline Num. arom. heavy atoms & 6 & & CYP2D6 inhibitor 8 & No \\
\hline Fraction Csp3 & 0.50 & & CYP3A4 inhibitor 1 & No \\
\hline $\begin{array}{l}\text { Num. rotatable bonds } \\
\text { Num. H-bond accentors }\end{array}$ & 4 & & $\log K_{p}$ (skin permeation) 0 & $-7.23 \mathrm{~cm} / \mathrm{s}$ \\
\hline $\begin{array}{l}\text { Num. H-bond acceptors } \\
\text { Num. H-bond donors }\end{array}$ & $\begin{array}{l}3 \\
1\end{array}$ & & & Druglikeness \\
\hline Molar Refractivity & 97.72 & & Lipinski 0 & Yes; 0 violation \\
\hline TPSA 8 & $69.72 \AA^{2}$ & & Ghose 0 & Yes \\
\hline & Lipophilicity & & Veber ${ }^{2}$ & Yes \\
\hline $\log P_{o / w}$ (iLOGP) & 3.02 & & Egan 8 & Yes \\
\hline $\log P_{\text {o/w }}($ XLOGP3) & 1.52 & & Muegge $\odot$ & Yes \\
\hline $\log P_{\text {o/w }}$ (WLOGP) & 0.53 & & Bioavailability Score & $\begin{array}{l}0.55 \\
\text { Medicinal Chemistry }\end{array}$ \\
\hline $\log P_{\mathrm{o} / \mathrm{w}}(\mathrm{MLOGP}) \odot$ & 1.12 & & PAINS 0 & $\begin{array}{l}\text { Medicinal Chemistry } \\
0 \text { alert }\end{array}$ \\
\hline $\log P_{\text {o/w }}($ SILICOS-IT) $)$ & 1.74 & & Brenk 8 & 1 alert: beta_keto_anhydride 0 \\
\hline Consensus Log $P_{\mathrm{o} / \mathrm{w}}$ (C) & 1.59 & & Leadlikeness (2) & Yes \\
\hline & & & Synthetic accessibility 8 & 3.07 \\
\hline
\end{tabular}




\section{$H \odot Q$}<smiles>CC1CCCN1C(=O)C1(C(=O)CC(C)(C)C)C(=O)N(c2ccccc2)c2ccccc21</smiles>

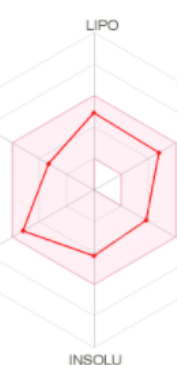

insolu
SMILES $\mathrm{O}=\mathrm{C} 1 \mathrm{CCCN} 1 \mathrm{C} 1(\mathrm{C}(=\mathrm{O}) \mathrm{NC}(\mathrm{C})(\mathrm{C}) \mathrm{C}) \mathrm{c} 2 \operatorname{ccccc} 2 \mathrm{~N}(\mathrm{C} 1=\mathrm{O}) \mathrm{c} 1 \mathrm{ccccc} 1$ Physicochemical Properties

\section{Formula}

C23H $25 \mathrm{~N} 3 \mathrm{O} 3$

Molecular weight $\quad 391.46 \mathrm{~g} / \mathrm{mol}$

Num. heavy atoms

29

Num. arom. heavy atoms

Fraction Csp3

12

Num. rotatable bonds $\quad 5$

Num. H-bond acceptors 3

Num. H-bond donors $\quad 1$

Molar Refractivity $\quad 117.96$

TPSA $969.72 \AA^{2}$

$\log P_{\text {O/w }}$ (iLOGP) $P$ Lipophilicity

$\log P_{0 / W}($ XLOGP3) 3.08

$\log P_{\mathrm{o} / \mathrm{w}}(\mathrm{WLOGP}) 02.23$

$\log P_{\mathrm{o} / \mathrm{w}}(\mathrm{MLOGP}) \odot 2.27$

$\log P_{\mathrm{o} / \mathrm{w}}(\mathrm{SILICOS}-\mathrm{IT}) \odot 2.84$

Consensus Log $P_{\mathrm{o} / \mathrm{w}} \odot \quad 2.78$

Water Solubility

Water Solubility

$\log S(E S O L)+-4.18$

SIZE Class 8

$2.56 \mathrm{e}-02 \mathrm{mg} / \mathrm{ml} ; 6.55 \mathrm{e}-05 \mathrm{~mol}$

$\log S$ (Ali)

Solubility

Moderately soluble

Class 8

$2.41 \mathrm{e}-02 \mathrm{mg} / \mathrm{ml} ; 6.15 \mathrm{e}-05 \mathrm{~mol} / \mathrm{l}$

$\log S$ (SILICOS-IT) 9

Moderately soluble

POLAR

Solubility

$-6.15$

Class Poorly soluble

\begin{tabular}{|c|c|}
\hline \multicolumn{2}{|r|}{ Pharmacokinetics } \\
\hline Gl absorption $(2)$ & High \\
\hline BBB permeant $(2$ & Yes \\
\hline P-gp substrate 2 & Yes \\
\hline CYP1A2 inhibitor $(8$ & No \\
\hline CYP2C19 inhibitor 2 & Yes \\
\hline CYP2C9 inhibitor $(3)$ & Yes \\
\hline CYP2D6 inhibitor 18 & Yes \\
\hline CYP3A4 inhibitor 2 & Yes \\
\hline Log $K_{\mathrm{p}}$ (skin permeation) & $-6.50 \mathrm{~cm} / \mathrm{s}$ \\
\hline \multicolumn{2}{|r|}{ Druglikeness } \\
\hline Lipinski 2 & Yes; 0 violation \\
\hline Ghose 2 & Yes \\
\hline Veber 8 & Yes \\
\hline Egan 8 & Yes \\
\hline Muegge 3 & Yes \\
\hline Bioavailability Score & 0.55 \\
\hline \multicolumn{2}{|r|}{ Medicinal Chemistry } \\
\hline PAINS 0 & 0 alert \\
\hline Brenk 9 & 1 alert: beta_keto_anhydride 3 \\
\hline Leadlikeness 0 & No; 1 violation: $M W>350$ \\
\hline Synthetic accessibility 0 & 3.52 \\
\hline
\end{tabular}

\section{$5 e a b$}

\section{$H \odot Q$}

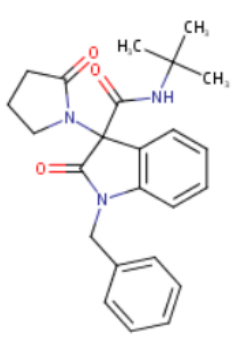

FLEX

INSATU

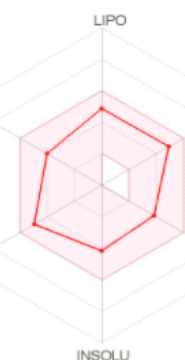

SMILES O $=\mathrm{C} 1 \mathrm{CCCN} 1 \mathrm{C} 1(\mathrm{C}(=\mathrm{O}) \mathrm{NC}(\mathrm{C})(\mathrm{C}) \mathrm{C}) \mathrm{C}(=\mathrm{O}) \mathrm{N}(\mathrm{c} 2 \mathrm{c} 1 \mathrm{cccc} 2) \mathrm{Cc} 1 \mathrm{ccccc} 1$ Physicochemical Properties

$\begin{array}{ll}\text { Formula } & \mathrm{C} 24 \mathrm{H} 27 \mathrm{~N} 3 \mathrm{O} 3 \\ \text { Molecular weight } & 405.49 \mathrm{~g} / \mathrm{mol} \\ \text { Num. heavy atoms } & 30 \\ \text { Num. arom. heavy atoms } & 12 \\ \text { Fraction Csp3 } & 0.38 \\ \text { Num. rotatable bonds } & 6 \\ \text { Num. H-bond acceptors } & 3 \\ \text { Num. H-bond donors } & 1 \\ \text { Molar Refractivity } & 122.21 \\ \text { TPSA } 9 & 69.72 \AA^{2}\end{array}$

Lipophilicity

$\log P_{\mathrm{o} / \mathrm{w}}$ (iLOGP) 3.26

$\log P_{\text {o/w }}($ XLOGP3) $\quad 3.01$

$\log P_{\mathrm{o} / \mathrm{w}}(\mathrm{WLOGP}){ }^{0} \quad 1.94$

$\log P_{\mathrm{o} / \mathrm{W}}$ (MLOGP) 2.21

$\log P_{\mathrm{o} / \mathrm{W}}($ SILICOS-IT) $\odot \quad 3.23$

Consensus Log $P_{\mathrm{o} / \mathrm{w}} \odot \quad 2.73$
Water Solubility

$-4.15$

$2.87 \mathrm{e}-02 \mathrm{mg} / \mathrm{ml} ; 7.07 \mathrm{e}-05 \mathrm{~mol} / \mathrm{l}$

Moderately soluble

SIZE Class 0

$-4.14$

$\log S$ (Ali) 6

$2.95 \mathrm{e}-02 \mathrm{mg} / \mathrm{ml} ; 7.26 \mathrm{e}-05 \mathrm{~mol} / \mathrm{l}$

Solubility

oderately soluble

$\log S$ (SILICOS-IT) $9-6.55$

POLAR Solubility $1.15 \mathrm{e}-04 \mathrm{mg} / \mathrm{ml} ; 2.84 \mathrm{e}-07 \mathrm{~mol} /$

Class $12 \quad$ Poorly soluble

Pharmacokinetics

Gl absorption 2 High

BBB permeant 18 Yes

P-gp substrate (2) Yes

CYP1A2 inhibitor $2 \quad$ No

CYP2C19 inhibitor 19 Yes

CYP2C9 inhibitor 9 Yes

CYP2D6 inhibitor 2 Yes

CYP3A4 inhibitor 9 Yes

Log $K_{\mathrm{p}}$ (skin permeation) $-6.64 \mathrm{~cm} / \mathrm{s}$

\begin{tabular}{ll} 
& Druglikeness \\
\hline Lipinski $\odot$ & Yes; 0 violation \\
Ghose $\odot$ & Yes \\
Veber $\odot$ & Yes \\
Egan $\odot$ & Yes \\
Muegge $\odot$ & Yes \\
Bioavailability Score $\odot$ & 0.55 \\
\hline & Medicinal Chemistry \\
PAINS $\odot$ & 0 alert \\
Brenk $\odot$ & 1 alert: beta_keto_anhydride $\odot$ \\
Leadlikeness $\odot$ & No; 1 violation: MW $>350$ \\
Synthetic accessibility $\odot$ & 3.53
\end{tabular}


H०Q<smiles>CC(C)(C)NC(=O)C1(N2CCCC2=O)C(=O)Nc2ccc(Br)cc21</smiles>

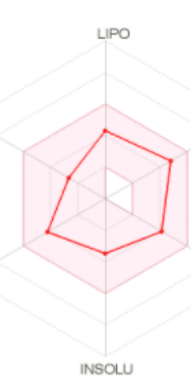

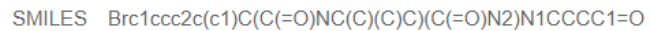
Physicochemical Properties

Formula $\mathrm{C} 17 \mathrm{H} 2 \mathrm{OBrN} 3 \mathrm{O} 3$

Molecular weight

$394.26 \mathrm{~g} / \mathrm{mol}$

Num. heavy atoms

24

Num. arom. heavy atoms

Fraction Csp3

6

0.47

Num. rotatable bonds $\quad 4$

Num. H-bond acceptors

Num. H-bond donors 2

Molar Refractivity $\quad 100.52$

TPSA $978.51 \AA^{2}$

$\log P_{\mathrm{o} / \mathrm{w}}$ (iLOGP) 2.28

$\log P_{\mathrm{o} / \mathrm{w}}(\mathrm{XLOGP} 3) \odot \quad 2.03$

$\log P_{\mathrm{o} / \mathrm{w}}$ (WLOGP) 1.07

$\log P_{\mathrm{o} / \mathrm{w}}(\mathrm{MLOGP}) \odot \quad 1.50$

$\log P_{\mathrm{o} / \mathrm{w}}($ SILICOS-IT) $\odot \quad 2.48$

Consensus $\log P_{\mathrm{o} / \mathrm{w}} \odot \quad 1.87$

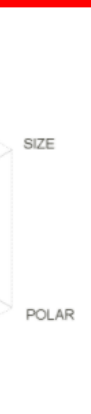

Solubility $\quad 1.29 \mathrm{e}-01 \mathrm{mg} / \mathrm{ml} ; 3.28 \mathrm{e}-04 \mathrm{~mol} / \mathrm{l}$

Class (2) Soluble

$\log S$ (Ali) 20

Solubility $\quad 1.95 \mathrm{e}-01 \mathrm{mg} / \mathrm{ml} ; 4.94 \mathrm{e}-04 \mathrm{~mol} / \mathrm{l}$

Class 18 Soluble

Log S (SILICOS-IT) $30 \quad-5.20$

Solubility $\quad 2.48 \mathrm{e}-03 \mathrm{mg} / \mathrm{ml} ; 6.30 \mathrm{e}-06 \mathrm{~mol} / \mathrm{l}$

Class (- Moderately soluble

Pharmacokinetics

Gl absorption $(2)$ High

BBB permeant 9 No

P-gp substrate $0 \quad$ Yes

CYP1A2 inhibitor (-) No

CYP2C19 inhibitor 2 Yes

CYP2C9 inhibitor 9

CYP2D6 inhibitor 18 No

CYP3A4 inhibitor 9 Yes

Log $K_{\mathrm{p}}$ (skin permeation) $-7.26 \mathrm{~cm} / \mathrm{s}$

\begin{tabular}{|c|c|}
\hline \multicolumn{2}{|r|}{ Druglikeness } \\
\hline Lipinski $\odot$ & Yes; 0 violation \\
\hline Ghose ? & Yes \\
\hline Veber $(3)$ & Yes \\
\hline Egan 0 & Yes \\
\hline Muegge 9 & Yes \\
\hline Bioavailability Score 2 & 0.55 \\
\hline \multicolumn{2}{|r|}{ Medicinal Chemistry } \\
\hline PAINS 8 & 0 alert \\
\hline Brenk 2 & 1 alert: beta_keto_anhydride $(2)$ \\
\hline Leadlikeness 3 & No; 1 violation: $M W>350$ \\
\hline Synthetic accessibility & 3.16 \\
\hline
\end{tabular}

\section{5 gab}

\section{$H \odot 0$}<smiles>CC(C)(C)NC(=O)C1(N2CCCC2=O)C(=O)Nc2ccc(I)cc21</smiles>

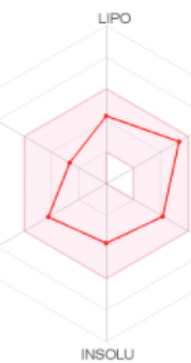

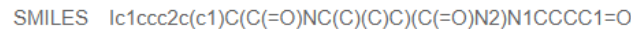

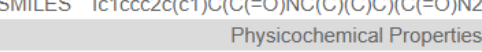$$
\text { Formula }
$$$$
\text { C17H2OIN3O3 }
$$

Molecular weight

$$
441.26 \mathrm{~g} / \mathrm{mol}
$$

Num. heavy atoms

24

Num. arom. heavy atoms

Fraction Csp3

0.47

Num. rotatable bonds

Num. H-bond acceptors

Num. H-bond donors

Molar Refractivity

TPSA B

4

3

2

105.53

$78.51 \AA^{2}$

$\log P_{\text {o/w }}($ iLOGP) $\odot 2.31$

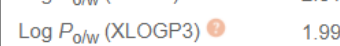

$\log P_{\mathrm{o} / \mathrm{w}}($ WLOGP) $\odot \quad 0.91$

$\log P_{\mathrm{o} / \mathrm{w}}(\mathrm{MLOGP}) \odot 1.62$

Log $P_{\text {o/w }}$ (SILICOS-IT) $0 \quad 2.77$

Consensus $\log P_{\mathrm{o} / \mathrm{w}} \odot \quad 1.92$
Synthetic accessibility 0 


\begin{tabular}{|c|c|c|c|}
\hline \multicolumn{4}{|l|}{ 5hab } \\
\hline \multirow{11}{*}{$H \odot Q$} & & & Water Solubility \\
\hline & UPO & $\log S(E S O L)$ & -2.65 \\
\hline & & Solubility & $8.15 \mathrm{e}-01 \mathrm{mg} / \mathrm{ml} ; 2.26 \mathrm{e}-03 \mathrm{~mol} / \mathrm{l}$ \\
\hline & FLEX & Class 0 & Soluble \\
\hline & & $\log S($ Ali) $\odot$ & -3.37 \\
\hline & & Solubility & $1.55 \mathrm{e}-01 \mathrm{mg} / \mathrm{ml} ; 4.31 \mathrm{e}-04 \mathrm{~mol} / \mathrm{l}$ \\
\hline & & Class ${ }^{2}$ & Soluble \\
\hline & & $\log S($ SILICOS-IT) $\odot$ & -3.76 \\
\hline & INSATU & $\begin{array}{l}\text { Solubility } \\
\text { Class } 0\end{array}$ & $\begin{array}{l}6.32 \mathrm{e}-02 \mathrm{mg} / \mathrm{ml} ; 1.75 \mathrm{e}-04 \mathrm{~mol} / \mathrm{l} \\
\text { Soluble }\end{array}$ \\
\hline & INsou & & Pharmacokinetics \\
\hline & & Gl absorption 8 & High \\
\hline \multicolumn{2}{|c|}{ SMILES $\mathrm{O}=\mathrm{C} 1 \mathrm{CCCN} 1 \mathrm{C} 1(\mathrm{C}(=\mathrm{O}) \mathrm{NC}(\mathrm{C})(\mathrm{C}) \mathrm{C}) \mathrm{C}(=\mathrm{O}) \mathrm{Nc} 2 \mathrm{c} 1 \mathrm{cc}(\mathrm{cc} 2)[\mathrm{N}+](=\mathrm{O})[\mathrm{O}-]$} & BBB permeant 0 & No \\
\hline \multicolumn{2}{|c|}{ Physicochemical Properties } & P-gp substrate $(2$ & Yes \\
\hline Formula & $\mathrm{C} 17 \mathrm{H} 20 \mathrm{~N} 4 \mathrm{O} 5$ & CYP1A2 inhibitor $\odot$ & No \\
\hline Molecular weight & $360.36 \mathrm{~g} / \mathrm{mol}$ & CYP2C19 inhibitor 0 & No \\
\hline Num. heavy atoms & 26 & CYP2C9 inhibitor 9 & No \\
\hline Num. arom. heavy atoms & 6 & CYP2D6 inhibitor 8 & No \\
\hline Fraction Csp3 & 0.47 & CYP3A4 inhibitor $\odot$ & No \\
\hline Num. rotatable bonds & $\begin{array}{l}5 \\
5\end{array}$ & $\log K_{p}$ (skin permeation) 0 & $-7.67 \mathrm{~cm} / \mathrm{s}$ \\
\hline $\begin{array}{l}\text { Num. H-bond acceptors } \\
\text { Num. H-bond donors }\end{array}$ & $\begin{array}{l}5 \\
2\end{array}$ & \multicolumn{2}{|r|}{ Druglikeness } \\
\hline Molar Refractivity & 101.64 & Lipinski $\odot$ & Yes; 0 violation \\
\hline \multirow[t]{2}{*}{ TPSA 0} & $124.33 \AA^{2}$ & Ghose 0 & Yes \\
\hline & Lipophilicity & Veber 8 & Yes \\
\hline $\log P_{\mathrm{o} / \mathrm{w}}$ (iLOGP) 0 & 1.94 & Egan $\odot$ & Yes \\
\hline $\log P_{\mathrm{o} / \mathrm{w}}(\mathrm{XLOGP} 3)$ & 1.16 & $\begin{array}{l}\text { Muegge } \odot \\
\text { Bioavailability Score } 8\end{array}$ & $\begin{array}{l}\text { Yes } \\
0.55\end{array}$ \\
\hline $\log P_{\mathrm{o} / \mathrm{W}}(\mathrm{WLOGP}) \odot$ & 0.22 & \multicolumn{2}{|r|}{ Medicinal Chemistry } \\
\hline $\log P_{\mathrm{o} / \mathrm{w}}(\mathrm{MLOGP}) \odot$ & -0.01 & PAINS $\odot$ & 0 alert \\
\hline $\log P_{\mathrm{o} / \mathrm{W}}(\mathrm{SILICOS}-\mathrm{IT}) \Theta$ & -0.33 & Brenk 2 & $\begin{array}{l}3 \text { alerts: beta_keto_anhydride, nitro_group, } \\
\text { oxygen-nitrogen_single_bond }\end{array}$ \\
\hline \multirow[t]{2}{*}{ Consensus Log $P_{o / w} \odot$} & 0.59 & Leadlikeness @ & No; 1 violation: $\mathrm{MW}>350$ \\
\hline & & Synthetic accessibility 0 & 3.31 \\
\hline
\end{tabular}

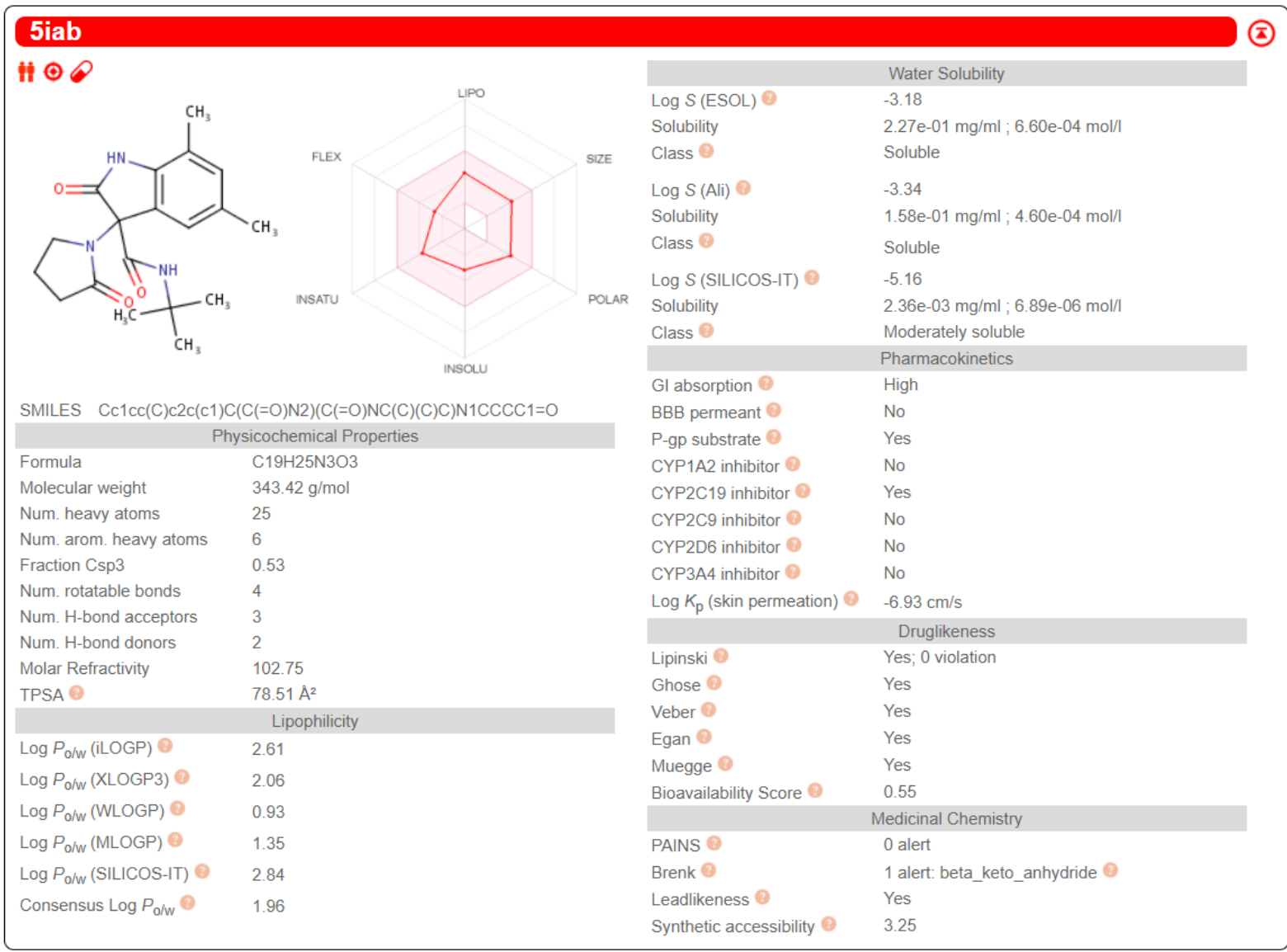


$+\odot Q$<smiles>CC(C)(C)NC(=O)C1(N2CCCC2=O)C(=O)Nc2c(C(F)(F)F)cccc21</smiles>

FLEX

NSATU

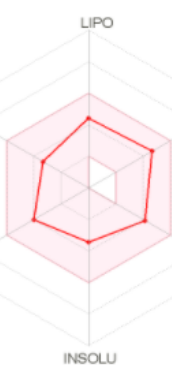

INSOLU
SMILES $\mathrm{O}=\mathrm{C} 1 \mathrm{CCCN} 1 \mathrm{C} 1(\mathrm{C}(=\mathrm{O}) \mathrm{NC}(\mathrm{C})(\mathrm{C}) \mathrm{C}) \mathrm{C}(=\mathrm{O}) \mathrm{Nc} 2 \mathrm{c} 1 \mathrm{cccc} 2 \mathrm{C}(\mathrm{F})(\mathrm{F}) \mathrm{F}$ Physicochemical Properties

Molecular weigh

$\mathrm{C} 18 \mathrm{H} 2 \mathrm{OF} 3 \mathrm{~N} 3 \mathrm{O} 3$

Num. heavy atoms

$383.36 \mathrm{~g} / \mathrm{mol}$

Num. arom. heavy atoms

27

oms 6

Num. rotatable bonds $\quad 5$

Num. H-bond acceptors 6

Num. H-bond donors 2

Molar Refractivity $\quad 97.82$

TPSA $278.51 \AA^{2}$

$\log P_{\mathrm{o} / \mathrm{w}}$ (iLOGP) 2.44

$\log P_{\mathrm{o} / \mathrm{w}}(\mathrm{XLOGP} 3) 0$

$\log P_{\mathrm{o} / \mathrm{w}}(\mathrm{WLOGP}) \odot 2.48$

$\log P_{0 / w}($ MLOGP) 01.73

$\log P_{\mathrm{o} / \mathrm{w}}(\mathrm{SILICOS}-\mathrm{IT}) \odot 2.90$

Consensus Log $P_{\mathrm{o} / \mathrm{w}}$ (2) 2.35

Lipophilicity

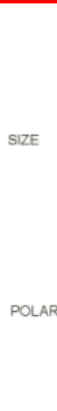

SIZE

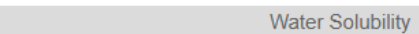

$\log S(E S O L)$

Solubility $\quad 1.36 \mathrm{e}-01 \mathrm{mg} / \mathrm{ml} ; 3.55 \mathrm{e}-04 \mathrm{~mol} / \mathrm{l}$

Class $(2 \quad$ Soluble

$\log S$ (Ali) $10 \quad-3.50$

Solubility $\quad 1.20 \mathrm{e}-01 \mathrm{mg} / \mathrm{ml} ; 3.14 \mathrm{e}-04 \mathrm{~mol} / \mathrm{l}$

Class 9 Soluble

Log S (SILICOS-IT) $10-5.25$

Solubility $\quad 2.18 \mathrm{e}-03 \mathrm{mg} / \mathrm{ml} ; 5.69 \mathrm{e}-06 \mathrm{~mol} / \mathrm{l}$

Class $12 \quad$ Moderately soluble

\begin{tabular}{|c|c|}
\hline \multicolumn{2}{|r|}{ Pharmacokinetics } \\
\hline Gl absorption (2) & High \\
\hline BBB permeant $(2$ & No \\
\hline P-gp substrate 2 & Yes \\
\hline CYP1A2 inhibitor $(2$ & No \\
\hline CYP2C19 inhibitor 9 & Yes \\
\hline CYP2C9 inhibitor 0 & No \\
\hline CYP2D6 inhibitor 2 & No \\
\hline CYP3A4 inhibitor 8 & Yes \\
\hline $\log K_{\mathrm{p}}$ (skin permeation) $\theta$ & $-7.06 \mathrm{~cm} / \mathrm{s}$ \\
\hline \multicolumn{2}{|r|}{ Druglikeness } \\
\hline Lipinski 8 & Yes; 0 violation \\
\hline Ghose 2 & Yes \\
\hline Veber 9 & Yes \\
\hline Egan $\odot$ & Yes \\
\hline Muegge 2 & Yes \\
\hline Bioavailability Score 2 & 0.55 \\
\hline \multicolumn{2}{|r|}{ Medicinal Chemistry } \\
\hline PAINS 0 & 0 alert \\
\hline Brenk 8 & 1 alert: beta_keto_anhydride \\
\hline Leadlikeness 8 & No; 1 violation: $M W>350$ \\
\hline Synthetic accessibility & 3.37 \\
\hline
\end{tabular}

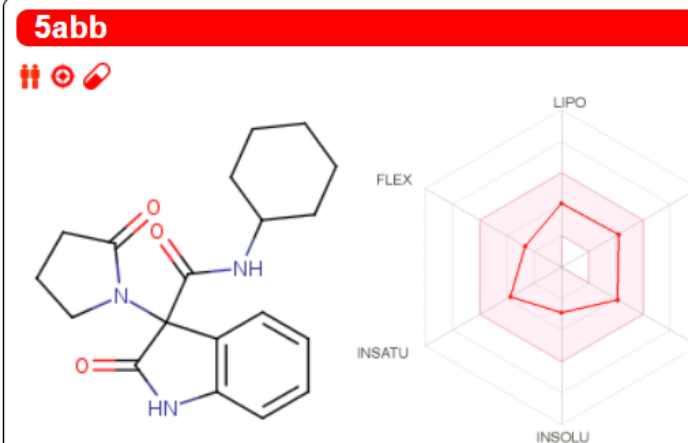

SMILES $\mathrm{O}=\mathrm{C} 1 \mathrm{CCCN} 1 \mathrm{C} 1(\mathrm{C}(=\mathrm{O}) \mathrm{NC} 2 \mathrm{CCCCC} 2) \mathrm{C}(=\mathrm{O}) \mathrm{Nc} 2 \mathrm{c} 1 \mathrm{cccc} 2$ Physicochemical Properties

\begin{tabular}{|c|c|}
\hline Formula & $\mathrm{C} 19 \mathrm{H} 23 \mathrm{~N} 3 \mathrm{O} 3$ \\
\hline Molecular weight & $341.40 \mathrm{~g} / \mathrm{mol}$ \\
\hline Num. heavy atoms & 25 \\
\hline Num. arom. heavy atoms & 6 \\
\hline Fraction Csp3 & 0.53 \\
\hline Num. rotatable bonds & 4 \\
\hline Num. H-bond acceptors & 3 \\
\hline Num. $\mathrm{H}$-bond donors & 2 \\
\hline Molar Refractivity & 100.28 \\
\hline \multirow[t]{2}{*}{ TPSA 8} & $78.51 \AA^{2}$ \\
\hline & Lipophilicity \\
\hline $\log P_{o / w}$ (iLOGP) 3 & 2.37 \\
\hline $\log P_{\mathrm{o} / \mathrm{w}}(\mathrm{XLOGP} 3)$ & 1.61 \\
\hline $\log P_{\mathrm{o} / \mathrm{w}}$ (WLOGP) & 0.84 \\
\hline $\log P_{\mathrm{o} / \mathrm{w}}$ (MLOGP) 8 & 1.35 \\
\hline $\log P_{\mathrm{o} / \mathrm{w}}($ SILICOS-IT) & 2.16 \\
\hline Consensus $\log P_{\mathrm{o} / \mathrm{w}}$ & 1.67 \\
\hline
\end{tabular}

Water Solubility

$-2.88$

$\begin{array}{ll}\text { Log } S(E S O L) & -2.88 \\ \text { Solubility } & 4.45 \mathrm{e}-01 \mathrm{mg} / \mathrm{ml} ; 1.30 \mathrm{e}-03 \mathrm{~mol} / \mathrm{l}\end{array}$

Class $2 \quad$ Soluble

Log S (Ali) $10 \quad-2.87$

Solubility $\quad 4.60 \mathrm{e}-01 \mathrm{mg} / \mathrm{ml} ; 1.35 \mathrm{e}-03 \mathrm{~mol} / \mathrm{l}$

Class $(2)$ Soluble

Log S (SILICOS-IT) 10

$8.28 \mathrm{e}-03 \mathrm{mg} / \mathrm{ml} ; 2.43 \mathrm{e}-05 \mathrm{~mol} / \mathrm{l}$

Class (2) Moderately soluble

Pharmacokinetics

High

BBB permeant 19 No

P-gp substrate 8 Yes

CYP1A2 inhibitor 9 No

CYP2C19 inhibitor 2 Yes

CYP2C9 inhibitor $2 \quad$ No

CYP2D6 inhibitor 9 No

CYP3A4 inhibitor 9 No

Log $K_{\mathrm{p}}$ (skin permeation) $\odot-7.24 \mathrm{~cm} / \mathrm{s}$

\begin{tabular}{ll} 
& Druglikeness \\
\hline Lipinski $\odot$ & Yes; 0 violation \\
Ghose $\odot$ & Yes \\
Veber $\odot$ & Yes \\
Egan $\odot$ & Yes \\
Muegge $\odot$ & Yes \\
Bioavailability Score $\odot$ & 0.55 \\
& Medicinal Chemistry \\
\hline PAINS $\odot$ & 0 alert \\
Brenk $\odot$ & 1 alert: beta_keto_anhydride $\odot$ \\
Leadlikeness $\odot$ & Yes \\
Synthetic accessibility $\odot$ & 3.12
\end{tabular}




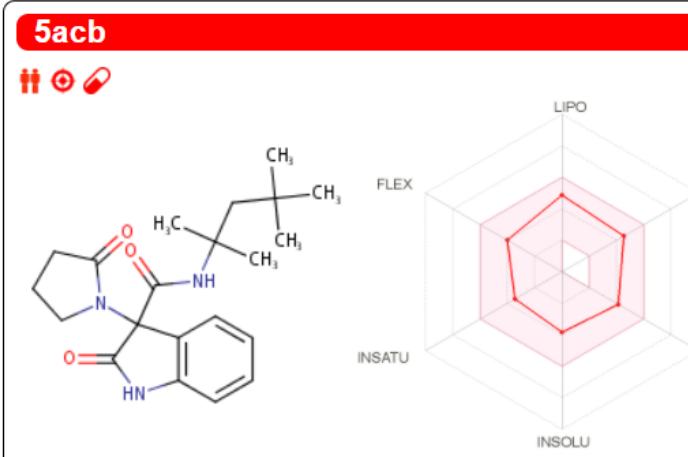

SMILES $\mathrm{O}=\mathrm{C} 1 \mathrm{CCCN} 1 \mathrm{C} 1(\mathrm{C}(=\mathrm{O}) \mathrm{NC}(\mathrm{CC}(\mathrm{C})(\mathrm{C}) \mathrm{C})(\mathrm{C}) \mathrm{C}) \mathrm{C}(=\mathrm{O}) \mathrm{Nc} 2 \mathrm{c} 1 \mathrm{cccc} 2$ Physicochemical Properties

\begin{tabular}{|c|c|}
\hline Formula & $\mathrm{C} 21 \mathrm{H} 29 \mathrm{~N} 3 \mathrm{O} 3$ \\
\hline Molecular weight & $371.47 \mathrm{~g} / \mathrm{mol}$ \\
\hline Num. heavy atoms & 27 \\
\hline Num. arom. heavy atoms & 6 \\
\hline Fraction Csp3 & 0.57 \\
\hline Num. rotatable bonds & 6 \\
\hline Num. H-bond acceptors & 3 \\
\hline Num. H-bond donors & 2 \\
\hline Molar Refractivity & 111.79 \\
\hline \multirow[t]{2}{*}{ TPSA 9} & $78.51 \AA^{2}$ \\
\hline & Lipophilicity \\
\hline $\log P_{\mathrm{o} / \mathrm{w}}$ (iLOGP) & 2.59 \\
\hline $\log P_{\mathrm{o} / \mathrm{w}}(\mathrm{XLOGP} 3)$ & 3.05 \\
\hline $\log P_{\mathrm{o} / \mathrm{w}}($ WLOGP) & 1.73 \\
\hline $\log P_{\mathrm{o} / \mathrm{w}}(\mathrm{MLOGP})$ & 1.80 \\
\hline $\log P_{\mathrm{o} / \mathrm{w}}($ SILICOS-IT) 0 & 3.06 \\
\hline Consensus Log $P_{\mathrm{o} / \mathrm{w}}$ & 2.44 \\
\hline
\end{tabular}

\section{5 adb}

$\# \odot \odot$<smiles>O=C1N=C2NC(=O)C1(N1CCCC1)c1ccccc1N2</smiles>

FLEX

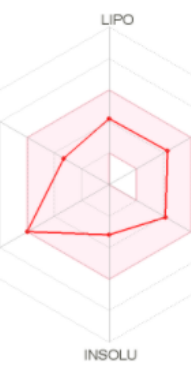

SMILES $\mathrm{O}=\mathrm{C} 1 \mathrm{CCCN} 1 \mathrm{C} 1(\mathrm{C}(=\mathrm{O}) \mathrm{NC} 2 \mathrm{ccccc} 2) \mathrm{C}(=\mathrm{O}) \mathrm{Nc} 2 \mathrm{c} 1 \mathrm{cccc} 2$ Physicochemical Properties

Formula $\mathrm{C} 2 \mathrm{OH} 19 \mathrm{~N} 3 \mathrm{O} 3$

Molecular weight

$349.38 \mathrm{~g} / \mathrm{mol}$

Num. heavy atoms

26

Num. arom. heavy atoms

Fraction Csp3

0.25

Num. rotatable bonds $\quad 5$

Num. H-bond acceptors 3

Num. H-bond donors 2

Molar Refractivity $\quad 102.85$

TPSA $978.51 \AA^{2}$

$\log P_{\mathrm{o} / \mathrm{w}}$ (iLOGP) 2.24

$\log P_{\text {o/w }}$ (XLOGP3) $\odot \quad 1.85$

$\log P_{\mathrm{o} / \mathrm{w}}$ (WLOGP) $\odot \quad 0.56$

$\log P_{\mathrm{o} / \mathrm{w}}$ (MLOGP) $2 \quad 1.35$

$\log P_{\text {o/w }}$ (SILICOS-IT) 202.44

Consensus Log $P_{\mathrm{o} / \mathrm{w}} \odot \quad 1.69$
SIZE

Solubility $\quad 5.46 \mathrm{e}-02 \mathrm{mg} / \mathrm{ml} ; 1.47 \mathrm{e}-04 \mathrm{~mol} / \mathrm{l}$

Class $(2) \quad$ Soluble

$\log S$ (Ali) $9-4.36$

Solubility $\quad 1.60 \mathrm{e}-02 \mathrm{mg} / \mathrm{ml} ; 4.32 \mathrm{e}-05 \mathrm{~mol} / \mathrm{l}$

Class $(2) \quad$ Moderately soluble

Log S (SILICOS-IT) $0 \quad-5.59$

POLAR

Solubility

Class 8

$9.44 \mathrm{e}-04 \mathrm{mg} / \mathrm{ml} ; 2.54 \mathrm{e}-06 \mathrm{~mol} / \mathrm{l}$

Moderately soluble

\begin{tabular}{|c|c|}
\hline \multicolumn{2}{|r|}{ Pharmacokinetics } \\
\hline Gl absorption 2 & High \\
\hline BBB permeant $(2$ & No \\
\hline P-gp substrate 2 & Yes \\
\hline CYP1A2 inhibitor $P$ & No \\
\hline CYP2C19 inhibitor 2 & Yes \\
\hline CYP2C9 inhibitor $(8)$ & No \\
\hline CYP2D6 inhibitor 9 & Yes \\
\hline CYP3A4 inhibitor 9 & Yes \\
\hline Log $K_{\mathrm{p}}$ (skin permeation) & $-6.40 \mathrm{~cm} / \mathrm{s}$ \\
\hline \multicolumn{2}{|r|}{ Druglikeness } \\
\hline Lipinski 2 & Yes; 0 violation \\
\hline Ghose 2 & Yes \\
\hline Veber 2 & Yes \\
\hline Egan 8 & Yes \\
\hline Muegge 9 & Yes \\
\hline Bioavailability Score 2 & 0.55 \\
\hline \multicolumn{2}{|r|}{ Medicinal Chemistry } \\
\hline PAINS 2 & 0 alert \\
\hline Brenk 2 & 1 alert: beta_keto_anhydride 2 \\
\hline Leadlikeness 9 & No; 1 violation: $M W>350$ \\
\hline Synthetic accessibility & 3.41 \\
\hline
\end{tabular}

Water Solubility

\begin{tabular}{ll} 
& Water Solubility \\
\hline & -3.18
\end{tabular}

Solubility $\quad 2.29 \mathrm{e}-01 \mathrm{mg} / \mathrm{ml} ; 6.56 \mathrm{e}-04 \mathrm{~mol}$

SIZE

Soluble

$\begin{array}{ll}\text { Class }(2) & \text { Soluble } \\ \text { Log } & -3.12\end{array}$

Solubility $\quad 2.65 \mathrm{e}-01 \mathrm{mg} / \mathrm{ml} ; 7.59 \mathrm{e}-04 \mathrm{~mol} / \mathrm{l}$

Class $(2) \quad$ Soluble

Log S (SILICOS-IT)

POLAR Solubility 2.81e- $04 \mathrm{mg} / \mathrm{ml} ; 8.04 \mathrm{e}-07 \mathrm{~mol} / \mathrm{l}$

Class (2) Poorly soluble

Pharmacokinetics

Gl absorption (2) High

BBB permeant $2 \quad$ No

P-gp substrate $2 \quad$ Yes

CYP1A2 inhibitor 9 No

CYP2C19 inhibitor 2 Yes

CYP2C9 inhibitor $2 \quad$ No

CYP2D6 inhibitor 2 Yes

CYP3A4 inhibitor 9 Yes

Log $K_{\mathrm{p}}$ (skin permeation) $3-7.12 \mathrm{~cm} / \mathrm{s}$

\begin{tabular}{|c|c|}
\hline \multicolumn{2}{|r|}{ Druglikeness } \\
\hline Lipinski 3 & Yes; 0 violation \\
\hline Ghose 9 & Yes \\
\hline Veber 9 & Yes \\
\hline Egan 8 & Yes \\
\hline Muegge 2 & Yes \\
\hline Bioavailability Score 2 & 0.55 \\
\hline \multicolumn{2}{|r|}{ Medicinal Chemistry } \\
\hline PAINS 8 & 0 alert \\
\hline Brenk 2 & 1 alert: beta_keto_anhydride \\
\hline Leadlikeness $(2$ & Yes \\
\hline Synthetic accessibility 3 & 3.01 \\
\hline
\end{tabular}




\section{$5 \mathbf{b c b}$}

$H \odot Q$
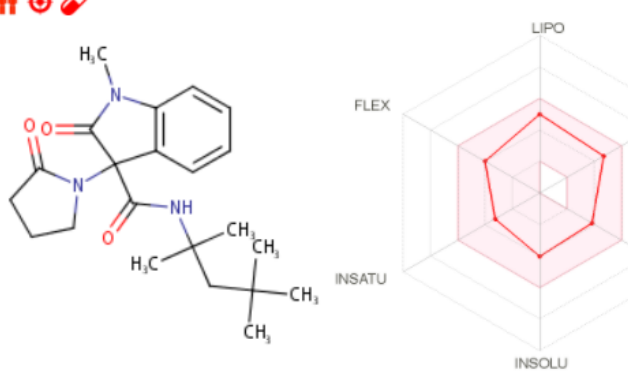

SMILES $\mathrm{O}=\mathrm{C} 1 \mathrm{CCCN} 1 \mathrm{C} 1(\mathrm{C}(=\mathrm{O}) \mathrm{NC}(\mathrm{CC}(\mathrm{C})(\mathrm{C}) \mathrm{C})(\mathrm{C}) \mathrm{C}) \operatorname{cocccc} 2 \mathrm{~N}(\mathrm{C} 1=\mathrm{O}) \mathrm{C}$ Physicochemical Properties

Formula

Molecular weight $\mathrm{C} 22 \mathrm{H} 31 \mathrm{~N} 3 \mathrm{O} 3$ $385.50 \mathrm{~g} / \mathrm{mol}$

Num. arom. heavy atoms

Fraction $\mathrm{Csp} 3$ 28

0.59

Num. rotatable bonds $\quad 6$

Num. H-bond acceptors 3

Num. H-bond donors $\quad 1$

Molar Refractivity $\quad 116.69$

Lipophilicity

$\log P_{\text {o/w }}($ iLOGP) 93.47

$\log P_{\mathrm{o} / \mathrm{W}}(\mathrm{XLOGP} 3) \odot 3.23$

$\log P_{\mathrm{o} / \mathrm{w}}(\mathrm{WLOGP}) \odot 1.94$

$\log P_{\mathrm{o} / \mathrm{w}}$ (MLOGP) 2.02

$\log P_{\mathrm{o} / \mathrm{w}}($ SILICOS-IT) 93.01

Consensus Log $P_{\text {o/w }} \odot \quad 2.73$
Num. heavy atoms

TPSA $969.72 \AA^{2}$

Water Solubility

$-4.03$

Solubility $\quad 3.62 \mathrm{e}-02 \mathrm{mg} / \mathrm{ml} ; 9.38 \mathrm{e}-05 \mathrm{~mol} / \mathrm{l}$

SIZE

Class $(2) \quad$ Moderately soluble

Log S (Ali) $18 \quad-4.37$

Solubility $\quad 1.66 \mathrm{e}-02 \mathrm{mg} / \mathrm{ml} ; 4.29 \mathrm{e}-05 \mathrm{~mol} / \mathrm{l}$

Class $(2) \quad$ Moderately soluble

Log S (SILICOS-IT)

Solubility $\quad 2.14 \mathrm{e}-03 \mathrm{mg} / \mathrm{ml} ; 5.55 \mathrm{e}-06 \mathrm{~mol} / \mathrm{l}$

Class 1 Moderately soluble

Pharmacokinetics

Gl absorption 3 High

BBB permeant 9 Yes

P-gp substrate $2 \quad$ Yes

CYP1A2 inhibitor 9 No

CYP2C19 inhibitor 2 Yes

CYP2C9 inhibitor 9 No

CYP2D6 inhibitor $(2) \quad$ Yes

CYP3A4 inhibitor (2) Yes

$\log K_{\mathrm{p}}$ (skin permeation) $-6.36 \mathrm{~cm} / \mathrm{s}$

\begin{tabular}{|c|c|}
\hline \multicolumn{2}{|r|}{ Druglikeness } \\
\hline Lipinski $(2)$ & Yes; 0 violation \\
\hline Ghose 2 & Yes \\
\hline Veber 8 & Yes \\
\hline Egan 2 & Yes \\
\hline Muegge $(8)$ & Yes \\
\hline Bioavailability Score 8 & 0.55 \\
\hline \multicolumn{2}{|r|}{ Medicinal Chemistry } \\
\hline PAINS 2 & 0 alert \\
\hline Brenk 2 & 1 alert: beta_keto_anhydride \\
\hline Leadlikeness $(2)$ & No; 1 violation: $M W>350$ \\
\hline Synthetic accessibility & 3.52 \\
\hline
\end{tabular}

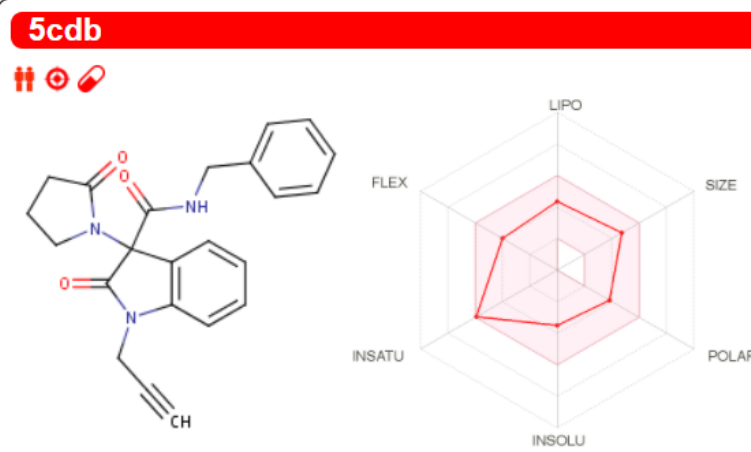

SMILES $\mathrm{C} \# \mathrm{CCN} 1 \mathrm{c} 2 \operatorname{ccccc} 2 \mathrm{C}(\mathrm{C} 1=\mathrm{O})(\mathrm{C}(=\mathrm{O}) \mathrm{NCc} 1 \mathrm{ccccc} 1) \mathrm{N} 1 \mathrm{CCCC} 1=\mathrm{O}$

\section{Physicochemical Properties}

Formula $\quad \mathrm{C} 23 \mathrm{H} 21 \mathrm{~N} 3 \mathrm{O} 3$

Molecular weight $\quad 387.43 \mathrm{~g} / \mathrm{mol}$

Num. heavy atoms $\quad 29$

Num. arom. heavy atoms $\quad 12$

Fraction Csp3 $\quad 0.26$

Num. rotatable bonds $\quad 6$

Num. H-bond acceptors 3

Num. H-bond donors $\quad 1$

Molar Refractivity $\quad 115.52$

TPSA $\left(69.72 \AA^{2}\right.$

Lipophilicity

$\log P_{\text {o/w }}$ (iLOGP) 192.82

$\log P_{\mathrm{o} / \mathrm{w}}(\mathrm{XLOGP} 3) \odot \quad 2.14$

$\log P_{\mathrm{o} / \mathrm{W}}(\mathrm{WLOGP}) \odot \quad 0.86$

$\log P_{\mathrm{o} / \mathrm{w}}(\mathrm{MLOGP})(1.93$

$\log P_{\text {o/w }}$ (SILICOS-IT) $\odot 2.99$

Consensus Log $P_{\text {o/w }} \odot \quad 2.15$

Water Solubility

$\log S(E S O L)(3.50$

Solubility $\quad 1.22 \mathrm{e}-01 \mathrm{mg} / \mathrm{ml} ; 3.16 \mathrm{e}-04 \mathrm{~mol} / /$

Class $1 \quad$ Soluble

Log $S$ (Ali) 19.24

Solubility $\quad 2.25 \mathrm{e}-01 \mathrm{mg} / \mathrm{ml} ; 5.81 \mathrm{e}-04 \mathrm{~mol} / \mathrm{l}$

Class $\theta^{-} \quad$ Soluble

$\log S$ (SILICOS-IT) $9-5.84$

Solubility $\quad 5.61 \mathrm{e}-04 \mathrm{mg} / \mathrm{ml} ; 1.45 \mathrm{e}-06 \mathrm{~mol} / \mathrm{l}$

Class $2 \quad$ Moderately soluble

Gl absorption $2 \quad$ High

BBB permeant 18 No

P-gp substrate $19 \quad$ Yes

CYP1A2 inhibitor 9 No

CYP2C19 inhibitor 9 Yes

CYP2C9 inhibitor (2) Yes

CYP2D6 inhibitor 9 Yes

CYP3A4 inhibitor 2 Yes

Log $K_{\mathrm{p}}$ (skin permeation) $-7.14 \mathrm{~cm} / \mathrm{s}$

\begin{tabular}{|c|c|}
\hline \multicolumn{2}{|r|}{ Druglikeness } \\
\hline Lipinski $\odot$ & Yes; 0 violation \\
\hline Ghose $(9$ & Yes \\
\hline Veber ${ }^{\circ}$ & Yes \\
\hline Egan 0 & Yes \\
\hline Muegge $\odot$ & Yes \\
\hline Bioavailability Score 2 & 0.55 \\
\hline \multicolumn{2}{|r|}{ Medicinal Chemistry } \\
\hline PAINS 0 & 0 alert \\
\hline Brenk $\odot$ & 2 alerts: beta_keto_anhydride, triple_bond $(8$ \\
\hline Leadlikeness 8 & No; 1 violation: $M W>350$ \\
\hline Synthetic accessibility 9 & 3.44 \\
\hline
\end{tabular}




\section{References}

1. Rainoldi, G.; Lesma, G.; Picozzi, C.; Lo Presti, L.; Silvani, A., One step access to oxindole-based -lactams through Ugi four-center three-component reaction. RSC Adv. 2018, 8 (61), 34903-34910.

2. Marques, C. S.; Burke, A. J., Enantioselective Rhodium(I)-Catalyzed Additions of Arylboronic Acids to N-1,2,3-Triazole-Isatin Derivatives: Accessing N-(1,2,3-Triazolmethyl)-3hydroxy-3-aryloxindoles. ChemCatChem 2016, 8 (22), 3518-3526.

3. Ellman, G. L.; Courtney, K. D.; Andres, V.; Featherstone, R. M., A new and rapid colorimetric determination of acetylcholinesterase activity. Biochemical Pharmacology 1961, 7 (2), 88-95.

4. $\quad$ Oliveira de Santana, Q. L.; Santos Evangelista, T. C.; Imhof, P.; Ferreira, S. B.; Fernández-Bolaños, J. G.; Sydnes, M. O.; Lopéz, Ó.; Lindbäck, E., Tacrine-sugar mimetic conjugates as enhanced cholinesterase inhibitors. Organic \& Biomolecular Chemistry 2021, 19 (10), 2322-2337.

5. Affini, A.; Hagenow, S.; Zivkovic, A.; Marco-Contelles, J.; Stark, H., Novel indanone derivatives as MAO B/H3R dual-targeting ligands for treatment of Parkinson's disease.

European Journal of Medicinal Chemistry 2018, 148, 487-497. 